\title{
VIRGINIA.
}

\section{PRIMARY TRAVERSE.}

Arringdale, Aylett, Beaver Dam, Bermuda Hundred, Bowling Green, Boykins, Camp Abraham Eustis, Camp Humphreys, Cape Henry, Charles City, Dinwiddie, Disputanta, Doswell, Emporia, Heathsville, Holland, Homeville, Ivor, Jarratt, Kara, Kilmarnock, King William, Lawrenceville, McKenney, Montross, Morattico, Moyock, New Kent, Newport News, Petersburg, Piney Point, Port Royal, Richmond, Smithfield, South Fill, Suffolk, Surry, Tappahannock, Toano, Urbanna, Virginia Beach, Wellville, White Plains, Williamsburg, and Yorktown quadrangles.

BRUNSWICK, CAROLINE, CHARLES CITY, DINWIDDIE, ESSEX, FAIRFAX, GREENSVILLE, HANOVER, ISLE OF WIGHT, JAMES CITY, KING AND OUEEN, KING WIHLIAM, MIDDLE: SEX, NANSEMOND, NEW KENT, PRINCE GEORGE, PRINCESS ANNE, SOUTHAMPTON, SURRY, SUSSEX, WARWICK, AND YORK COUNTIES.

The following geographic positions on North American datum were determined in 1916, 1917, and 1918, by J. J. Charters, J. C. Fales, F. J. McMaugh, E. L. McNair, J. J. Phelan, A. C. Schilling, H. J. Switzer, and J. H. Wilson, as indicated at the head of the several quadrangle lists.

For other positions in Virginia see U. S. Geological Survey Bulletins $122,181,245,310,440,496,551$, and 644 , and the unpublished records of the U. S. Coast and Geodetic Survey and the Chief of Engineers, U. S. Army.

ARRINGDALE QUADRANGLE.

Southwest along Southern Ry. through northwest corner of quadrangle. (By H. J. Switzer in 1918.)

\begin{tabular}{|c|c|c|c|c|c|c|}
\hline Station. & Lat & ude. & & ongit & tude. & $\begin{array}{l}\text { Distance } \\
\text { between }\end{array}$ \\
\hline 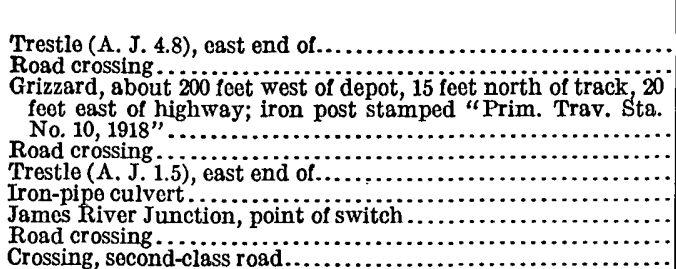 & $\begin{array}{lc}36 & 1 \\
36 & 44 \\
36 & 44 \\
& \\
36 & 43 \\
36 & 43 \\
36 & 42 \\
36 & 42 \\
36 & 42 \\
36 & 42 \\
36 & 42\end{array}$ & $\begin{array}{l}31 " \\
35.5 \\
02.5 \\
28.6 \\
23.0 \\
57.0 \\
29.0 \\
13.3 \\
13.0 \\
12.1\end{array}$ & $\begin{array}{l}77 \\
77 \\
77 \\
77 \\
77 \\
77 \\
77 \\
77 \\
77\end{array}$ & $\begin{array}{l}1 \\
24 \\
25 \\
26 \\
26 \\
27 \\
27 \\
28 \\
28 \\
29\end{array}$ & $\begin{array}{c}\prime \prime \\
17.0 \\
04.3 \\
\\
21.3 \\
42.9 \\
06.2 \\
50.6 \\
28.3 \\
41.2 \\
23.1\end{array}$ & $\begin{array}{l}7,145 \\
1,844 \\
3,250 \\
4,587 \\
3,461 \\
1,051 \\
3,413\end{array}$ \\
\hline
\end{tabular}

Along highways.

\begin{tabular}{|c|c|c|c|c|c|c|c|}
\hline 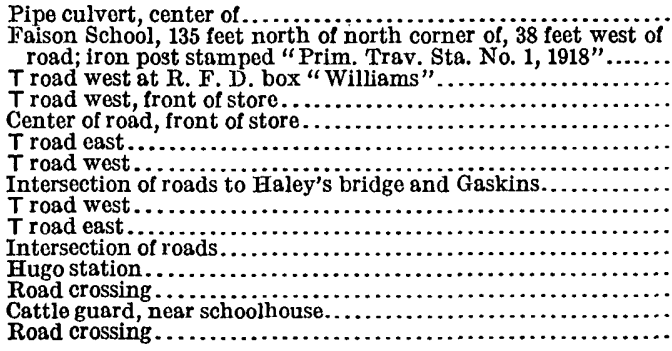 & $\begin{array}{l}36 \\
36 \\
36 \\
36 \\
36 \\
36 \\
36 \\
36 \\
36 \\
36 \\
36 \\
36 \\
36 \\
36 \\
36\end{array}$ & $\begin{array}{l}38 \\
37 \\
37 \\
36 \\
36 \\
35 \\
35 \\
34 \\
34 \\
34 \\
32 \\
33 \\
33 \\
33\end{array}$ & $\begin{array}{l}22.9 \\
54.9 \\
21.6 \\
47.0 \\
18.9 \\
43.6 \\
18.7 \\
48.1 \\
20.3 \\
10.8 \\
55.1 \\
08.0 \\
36.4 \\
52.7\end{array}$ & $\begin{array}{l}77 \\
77 \\
77 \\
77 \\
77 \\
77 \\
77 \\
77 \\
77 \\
77 \\
77 \\
77 \\
77 \\
77 \\
77\end{array}$ & $\begin{array}{l}28 \\
27 \\
27 \\
26 \\
25 \\
25 \\
24 \\
24 \\
22 \\
22 \\
17 \\
17 \\
16 \\
15\end{array}$ & $\begin{array}{l}32.0 \\
46.5 \\
04.8 \\
34.5 \\
56.3 \\
25.8 \\
57.1 \\
09.0 \\
44.6 \\
16.0 \\
49.5 \\
21.1 \\
18.7 \\
42.8\end{array}$ & \begin{tabular}{r}
7,353 \\
4,664 \\
4,790 \\
4,280 \\
4,219 \\
4,358 \\
3,437 \\
4,997 \\
7,432 \\
2,523 \\
\hdashline 2,653 \\
5,851 \\
3,360
\end{tabular} \\
\hline
\end{tabular}


ARRINGDALE QUADRANGIE-Continued. North along highways near east border of quadrangle.

\begin{tabular}{|c|c|c|c|c|c|c|c|}
\hline \multirow[t]{2}{*}{ Station. } & \multicolumn{3}{|c|}{ Latitude. } & \multicolumn{3}{|c|}{ Longitude. } & \multirow{2}{*}{$\begin{array}{c}\begin{array}{c}\text { Distance } \\
\text { between } \\
\text { stations. }\end{array} \\
\text { Feet. }\end{array}$} \\
\hline & $\circ$ & ' & $\prime \prime$ & $\circ$ & , & $\prime \prime$ & \\
\hline Culvert under road $\ldots \ldots \ldots \ldots \ldots \ldots \ldots \ldots \ldots \ldots$ & 36 & 34 & 37.3 & 77 & 16 & 13.8 & \\
\hline Culvert under road . ................. & 36 & 34 & 39.9 & 77 & 16 & 38.8 & 2,055 \\
\hline T road north...$\ldots \ldots \ldots \ldots \ldots \ldots \ldots \ldots \ldots \ldots \ldots \ldots \ldots$ & 36 & 34 & 38.1 & 77 & 17 & 09.0 & 2,468 \\
\hline 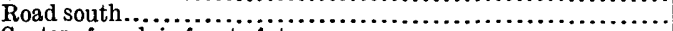 & 36 & 34 & 56.7 & 77 & 18 & 29.6 & 6,841 \\
\hline Center of road, in front of store . . . . . . & 36 & 35 & 24.7 & 77 & 19 & 19.6 & 4,962 \\
\hline 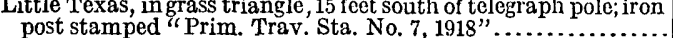 & 36 & 36 & 07.7 & 77 & 20 & 23.4 & 6,785 \\
\hline 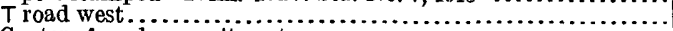 & 36 & 36 & 48.5 & 77 & 20 & 24.3 & 4,131 \\
\hline Center of road, opposite gate.. & 36 & 37 & 49.5 & 77 & 20 & 02.3 & 6,422 \\
\hline T road west................ & 36 & 38 & 09.0 & 77 & 19 & 41.9 & 2,585 \\
\hline 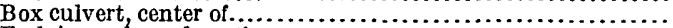 & 36 & 38 & 28.4 & 77 & 18 & 54.7 & 4,315 \\
\hline 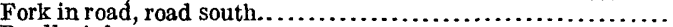 & 36 & 38 & 38.8 & 77 & 17 & 55.0 & 4,981 \\
\hline 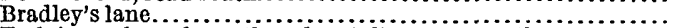 & 36 & 39 & 08.4 & 77 & 17 & 37.0 & 3,333 \\
\hline Fork in road, roads north and east, line turns north.......... & 36 & 39 & 23.6 & 77 & 17 & 18.0 & 2,185 \\
\hline 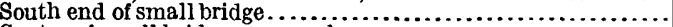 & 36 & 40 & 19.8 & 77 & 17 & 04.3 & 5,791 \\
\hline 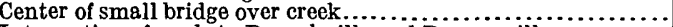 & 36 & 41 & 01.5 & 77 & 17 & 05.9 & 4,221 \\
\hline Intersection of roads to Branchville and Drewryville... & 36 & 41 & 41.8 & 77 & 17 & 07.8 & 4,080 \\
\hline Intersection of roads to Drewryville and Emporia............... & 36 & 42 & 16.3 & 77 & & 21.2 & 6,923 \\
\hline
\end{tabular}

West along Southern Ry. through north half of quadrangle.

Drewryville railroad station, 160 feet west of west end of, 10 feet south of south rail of south track; iron post stamped "Prim. Trav. Sta. No. 8, 1918"

Road crossing, north rail at.

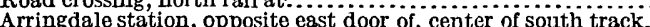

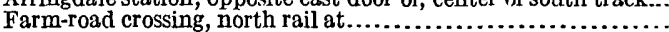

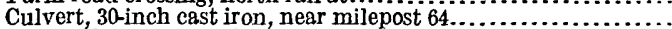

Adams Grove railroad station, 50 fcet west of west end of, 6 feet north of track; iron post stamped "Prim. Trav. Sta. No. 9, 1918 "

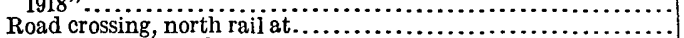

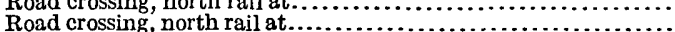

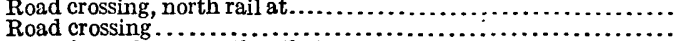

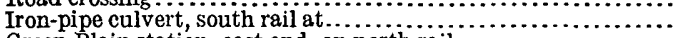

Green Plain station, east end, on north rail . .................

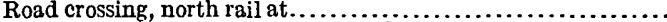

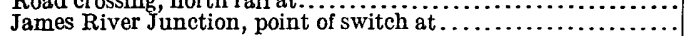

$\begin{array}{|lll|} & & \\ 36 & 42 & 56.6 \\ 36 & 42 & 58.8 \\ 36 & 43 & 02.3 \\ 36 & 42 & 22.4 \\ 36 & 41 & 50.3 \\ & & \\ 36 & 41 & 34.8 \\ 36 & 41 & 29.8 \\ 36 & 41 & 31.9 \\ 36 & 41 & 31.3 \\ 36 & 41 & 40.3 \\ 36 & 41 & 48.7 \\ 36 & 41 & 58.9 \\ 36 & 42 & 13.3\end{array}$

\begin{tabular}{|lll|l} 
& & & \\
77 & 18 & 28.7 & 4,126 \\
77 & 19 & 10.9 & 3,439 \\
77 & 20 & 14.4 & 5,180 \\
77 & 21 & 25.5 & 7,059 \\
77 & 22 & 27.5 & 6,002 \\
& & & \\
77 & 23 & 12.6 & 3,994 \\
77 & 23 & 31.0 & 1,585 \\
77 & 24 & 06.9 & 2,932 \\
77 & 24 & 53.9 & 3,826 \\
77 & 26 & 19.8 & 7,058 \\
77 & 27 & 09.0 & 4,092 \\
77 & 27 & 46.3 & 3,211 \\
77 & 28 & 28.3 & 3,718
\end{tabular}

Along highways near northeast corner of quadrangle.

Bridge 209 feet long, southeast end of (distance from Primary

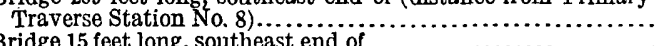
Bridge 15 feet long, southeast end of . .

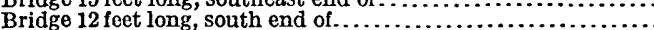

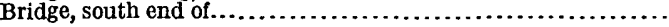

\begin{tabular}{|lll|lll|l}
36 & 43 & 16.3 & 77 & 18 & 34.0 & \\
36 & 43 & 43.3 & 77 & 19 & 08.0 & 2,037 \\
36 & 43 & 53.6 & 77 & 19 & 26.1 & 3,887 \\
36 & 44 & 44.2 & 77 & 19 & 22.4 & 1,807 \\
\hline
\end{tabular}

Along highways from Gaskins to Iittle Texas.

\begin{tabular}{|c|c|c|c|c|c|c|c|}
\hline 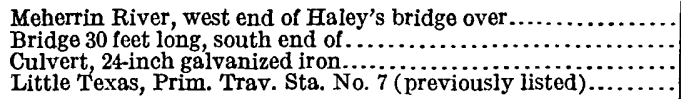 & $\begin{array}{l}36 \\
36 \\
36 \\
36\end{array}$ & $\begin{array}{l}34 \\
34 \\
35 \\
36\end{array}$ & $\begin{array}{l}12.0 \\
29.7 \\
09.0 \\
07.7\end{array}$ & $\begin{array}{l}77 \\
77 \\
77 \\
77\end{array}$ & $\begin{array}{l}21 \\
21 \\
21 \\
20\end{array}$ & $\begin{array}{l}43.0 \\
27.5 \\
15.4 \\
23.4\end{array}$ & $\begin{array}{l}2,701 \\
2,200 \\
4,106 \\
7,299\end{array}$ \\
\hline
\end{tabular}

North along highways near Meherrin River.

Meherrin River bridge, 1.10 miles from junction of GaskinsMargarettsville roads, 40 feet north of north end of bridge; iron

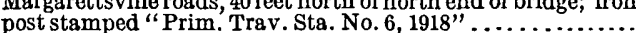

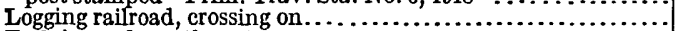

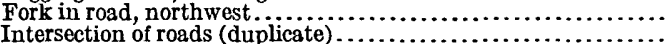

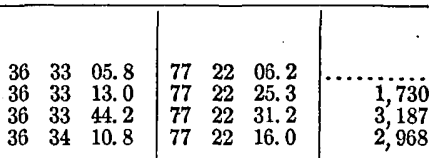

Magnetic declination for east border of quadrangle, $4^{\circ} 10^{\prime} \mathrm{W}$; for south border, $3^{\circ} 54^{\prime} \mathrm{W}$.; for center, $4^{\circ} 10^{\prime} \mathrm{W}$.; for southeast corner, $4^{\circ} 30^{\prime} \mathrm{W}$.

East along Southern Ry. (By J. J. Phelan in 1917.)

\begin{tabular}{|c|c|c|c|c|c|c|c|}
\hline 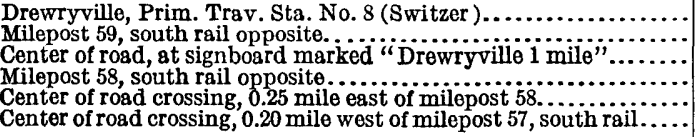 & $\begin{array}{l}36 \\
36 \\
36 \\
36 \\
36 \\
36\end{array}$ & $\begin{array}{l}42 \\
43 \\
43 \\
43 \\
43 \\
43\end{array}$ & $\begin{array}{l}56.6 \\
04.1 \\
06.7 \\
04.5 \\
05.7 \\
10.7\end{array}$ & $\begin{array}{l}77 \\
77 \\
77 \\
77 \\
77 \\
77\end{array}$ & $\begin{array}{l}18 \\
17 \\
17 \\
16 \\
16 \\
15\end{array}$ & $\begin{array}{l}28.7 \\
35.6 \\
18.9 \\
31.4 \\
14.5 \\
34.6\end{array}$ & $\begin{array}{r}4,3 \\
1,3\end{array}$ \\
\hline
\end{tabular}


ATLETT QUADRANGIE.

Along highways in extreme southwest corner of quadrangle. (By E. L. McIfair in 1916.)

\begin{tabular}{|c|c|c|c|}
\hline Station. & Latitude. & Longitude. & $\begin{array}{l}\text { Distance } \\
\text { betweon } \\
\text { stations. }\end{array}$ \\
\hline 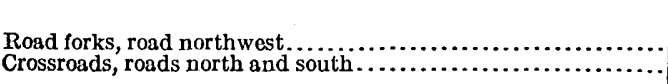 & $\begin{array}{ccc}\circ & \prime & \prime \prime \\
37 & 45 & 58.0 \\
37 & 45 & 25.1\end{array}$ & $\begin{array}{ccc}\circ & \prime & \prime \prime \\
77 & 14 & 42.6 \\
77 & 13 & 44.3\end{array}$ & $\begin{array}{c}\text { Feet. } \\
\cdots \cdots \\
\cdots, \ddot{\mathbf{5}} \ddot{\mathbf{4} 4}\end{array}$ \\
\hline
\end{tabular}

Along highways east to west through north half of quadrangle.

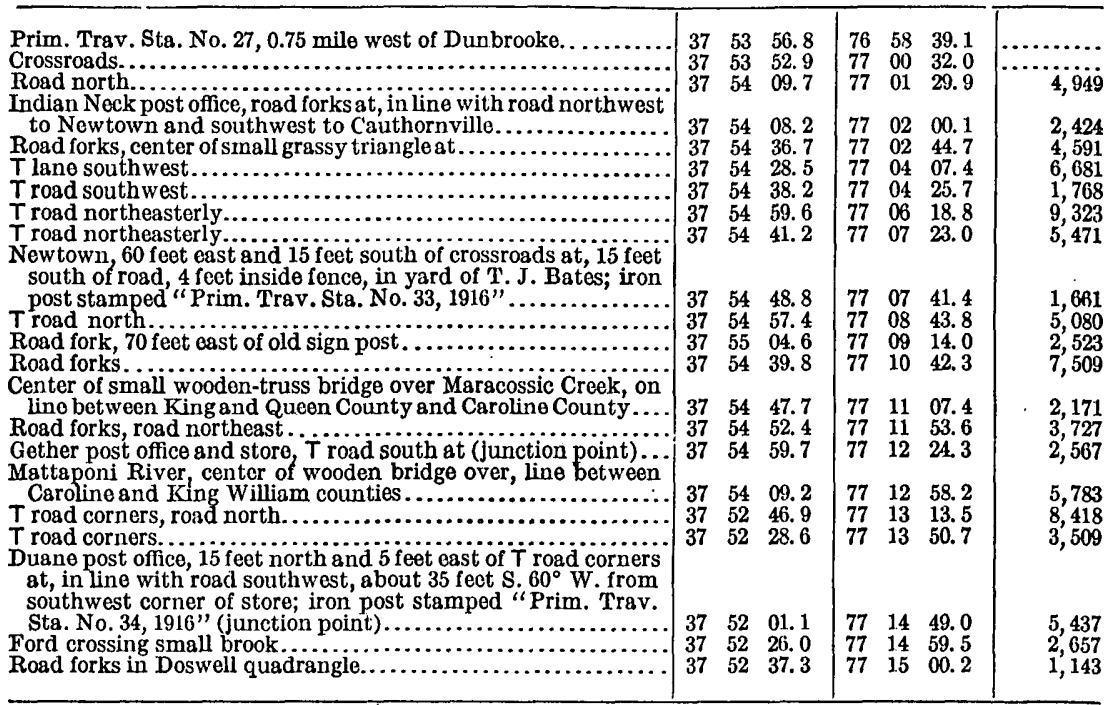

Magnetic declination for southwest corner of quadrangle, $5^{\circ} 00^{\prime} \mathrm{W}$.; for center of north half of quadrangle, $5^{\circ} 00^{\prime} \mathrm{W}$.

East along highways near north border of quadrangle.

Road forks.

Road forks, point is in line with roads in three directions.........

Sparta post office and store, 50 feet east of, 35 fect west of forks of road, across road opposite house of Dr. Campbell, on bank in front of house of Mrs. Rolfe (Rolfe estate); iron post stamped

"Prim. Trav. Sta. No. 40, 1916"............................

Mill race, center of small wooden bridge over...................

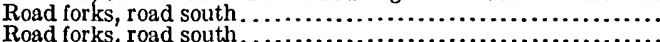

Road forks, road north.

Crossroads.

Alps post office, T road east at............................

Road forks. . .......................................

Small creek, center of wooden bridge over...................

Central Point, forks of road just cast of church at, about 50 feet

.east of line of road going south............................

north and 3 fect east of $T$ road corners, 1.5 feet inside fence in yard of M. W. Bird (colored); iron poststamped "Prim. Trav. yard of M. W. Bird (colored); iron post stamped "Prim. Trav.

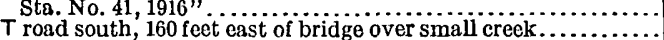
Road forks, at old signboard................................

Crossroads, road north and second-class road south............

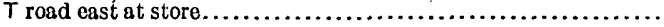

\begin{tabular}{|lll|lll|r}
37 & 59 & 20.8 & 77 & 14 & 38.5 & $\ldots \ldots, 3$, \\
37 & 59 & 26.6 & 77 & 14 & 22.2 & 1,428 \\
& & & & & & \\
& & & & & & \\
37 & 59 & 30.6 & 77 & 13 & 47.0 & 2,851 \\
37 & 59 & 25.2 & 77 & 12 & 53.2 & 4,338 \\
37 & 59 & 20.8 & 77 & 12 & 17.9 & 2,867 \\
37 & 59 & 28.8 & 77 & 11 & 46.7 & 2,622 \\
37 & 59 & 34.0 & 77 & 11 & 25.0 & 1,818 \\
37 & 59 & 40.9 & 77 & 10 & 48.6 & 3,000 \\
37 & 59 & 31.7 & 77 & 10 & 45.2 & 966 \\
37 & 59 & 22.7 & 77 & 09 & 51.6 & 4,394 \\
37 & 59 & 29.9 & 77 & 09 & 10.3 & 3,381 \\
& & & & & & \\
37 & 59 & 20.1 & 77 & 07 & 54.3 & 6,170 \\
& & & & & & \\
& & & & & & \\
37 & 59 & 26.9 & 77 & 07 & 44.8 & 1,023 \\
37 & 58 & 50.5 & 77 & 07 & 09.4 & 4,641 \\
37 & 58 & 32.6 & 77 & 06 & 27.2 & 3,834 \\
37 & 58 & 31.1 & 77 & 05 & 07.4 & 6,400 \\
37 & 58 & 29.2 & 77 & 04 & 12.4 & 4,405 \\
\hline
\end{tabular}


AYIETT QUADRANGLE-Continued.

South along highways near west border of north half of quadrangle.

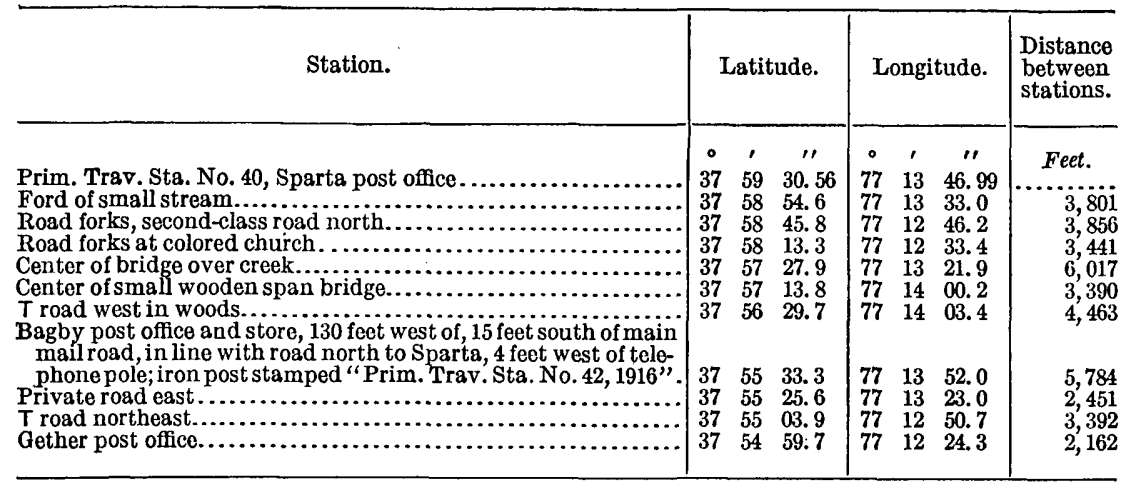

Along highway north-south through center of quadrangle.

\begin{tabular}{|c|c|c|c|c|c|c|c|}
\hline 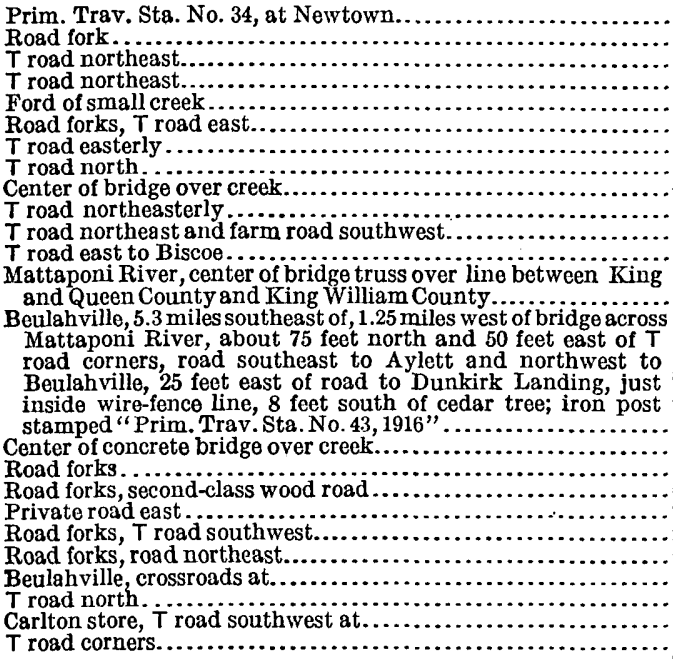 & $\begin{array}{l}37 \\
37 \\
37 \\
37 \\
37 \\
37 \\
37 \\
37 \\
37 \\
37 \\
37 \\
37\end{array}$ & $\begin{array}{l}54 \\
54 \\
52 \\
52 \\
52 \\
51 \\
51 \\
50 \\
50 \\
49 \\
49 \\
49\end{array}$ & $\begin{array}{l}48.8 \\
01.4 \\
51.2 \\
21.4 \\
16.4 \\
56.8 \\
02.4 \\
29.2 \\
08.1 \\
46.6 \\
20.9 \\
01.1 \\
48.8\end{array}$ & $\begin{array}{l}77 \\
77 \\
77 \\
77 \\
77 \\
77 \\
77 \\
77 \\
77 \\
77 \\
77 \\
77\end{array}$ & $\begin{array}{l}07 \\
08 \\
08 \\
08 \\
08 \\
07 \\
07 \\
07 \\
07 \\
06 \\
06 \\
05\end{array}$ & $\begin{array}{l}41.4 \\
01.0 \\
39.5 \\
10.1 \\
04.6 \\
48.4 \\
26.3 \\
11.9 \\
04.4 \\
33.9 \\
08.2 \\
53.5 \\
58.2\end{array}$ & $\begin{array}{r}5,039 \\
7,744 \\
3,828 \\
676 \\
2,366 \\
5,787 \\
3,547 \\
2,215 \\
3,273 \\
3,323 \\
2,324 \\
1,298\end{array}$ \\
\hline
\end{tabular}

Magnetic declination near north Dorder of quadrangle, $5^{\circ} 20^{\prime} \mathrm{W}$.; near center of quadrangle, $5^{\circ} 18^{\prime} \mathrm{W}$.; in southwest quarter of quadrangle, $5^{\circ} 00^{\prime} \mathrm{W}$.

Along highways in northeast quarter of quadrangle.

Lloyds post office, about 0.75 mile southeast of, 200 feet N. $64^{\circ}$ W. from northwest corner of Essex Church, in line with road west and close to forks of road; iron post stamped "Prim.

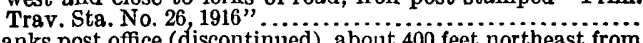
Banks post office (discontinued), about 400 feet northeast from

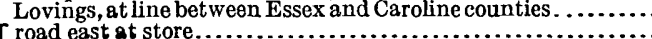

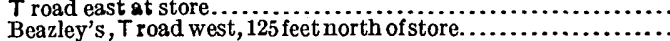

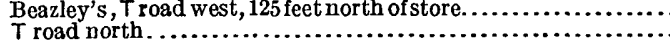
Road forks, 7 feet southeast of pine tree..........................

Road forks, road south . ..................................

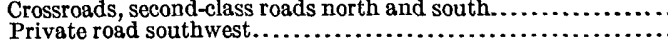

\begin{tabular}{|lll|lll|r}
\hline & & & & & & \\
37 & 59 & 28.8 & 77 & 00 & 17.8 & $\ldots \ldots \ldots \ldots$ \\
37 & 59 & 39.2 & 77 & 04 & 20.7 & $\ldots \ldots \ldots \ldots$ \\
37 & 58 & 29.2 & 77 & 04 & 12.4 & 7,108 \\
37 & 57 & 53.7 & 77 & 04 & 13.6 & 3,598 \\
37 & 57 & 34.3 & 77 & 02 & 53.8 & 6,686 \\
37 & 57 & 16.7 & 77 & 02 & 19.7 & 3,254 \\
37 & 56 & 52.2 & 77 & 01 & 44.3 & 3,766 \\
37 & 56 & 47.3 & 77 & 01 & 05.9 & 3,119 \\
37 & 56 & 47.6 & 77 & 00 & 29.5 & 2,911 \\
& & & & & \\
\hline
\end{tabular}

Magnetic declination for northeast quarter of quadrangle, $4^{\circ} 50^{\prime} \mathrm{W}$. 
Beaver daM gUadrangIe.

South along highways near east border of south half of quadrangle. (Bg. E. L. McNalr In 1916.)

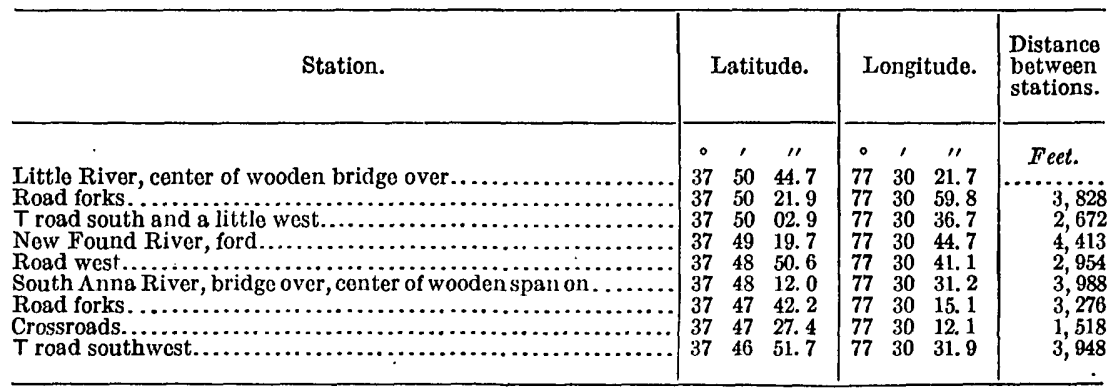

Magnetic declination for east border of south half of quadrangle, $5^{\circ} 00^{\prime} \mathrm{W}$.

BERMUDA HUNDRED QUADRANGLE.

Near east border of quadrangle. (By E. I. McNair in 1916.)

\begin{tabular}{|c|c|c|c|}
\hline Station. & Latitude. & Longitude. & $\begin{array}{l}\text { Distance } \\
\text { between } \\
\text { stations. }\end{array}$ \\
\hline $\begin{array}{l}\text { Malvern Hill post office, } 2 \text { miles northwest of, river road at road } \\
\text { northeast to Elko, at northwest corner; iron post stamped } \\
\text { "Prim. Trav. Sta. No. } 56,1916 " \ldots \ldots \ldots \ldots \ldots \ldots \ldots \ldots \ldots \ldots \ldots \ldots \ldots \ldots\end{array}$ & $\begin{array}{ccc}\circ & \prime & \prime \prime \\
37 & 24 & 20.2\end{array}$ & $\begin{array}{ccc}0 & \prime & \prime \prime \\
71 & 15 & 58.4\end{array}$ & $\begin{array}{l}\text { Feet. } \\
\text {. }\end{array}$ \\
\hline
\end{tabular}

Magnetic declination for east border of quadrangle, $4^{\circ} 13^{\prime} \mathrm{W}$.

\section{BOWLING GREEN QUADRANGLE.}

East along highways near south border of quadrangle (spur line). (By E. L. McNair in 1916.)

\begin{tabular}{|c|c|c|c|}
\hline Station. & Latitude. & Longitude. & $\begin{array}{l}\text { Distance } \\
\text { between } \\
\text { stations. }\end{array}$ \\
\hline Crossroads, second class......................... & $\begin{array}{ccc}\circ & \prime & \prime \prime \\
38 & 00 & 01.1\end{array}$ & $\begin{array}{ccc}\circ & \prime & \prime \prime \\
77 & 25 & 53.8\end{array}$ & Fect. \\
\hline
\end{tabular}

East along highways near south border of quadrangle (main circuit).

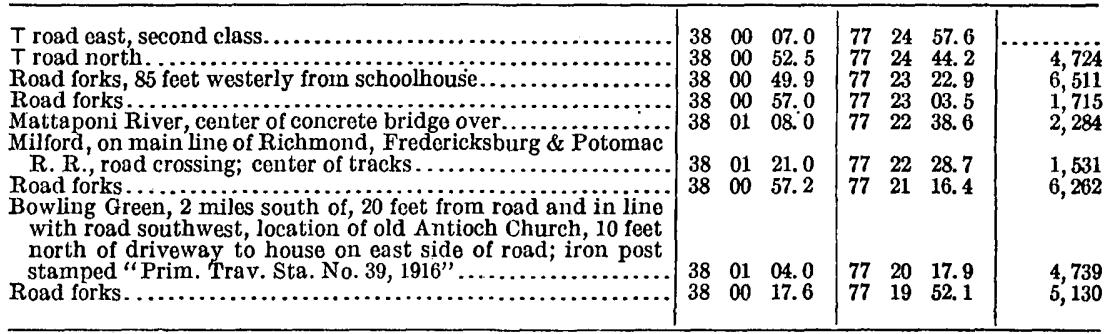

Magnetic declination near south border of quadrangle, $5^{\circ} 15^{\prime} \mathrm{W}$. 
BOYKINS QUADRANGLE.

Southwest along highways near east border of quadrangle. (By J. J. Phelan in 1918.)

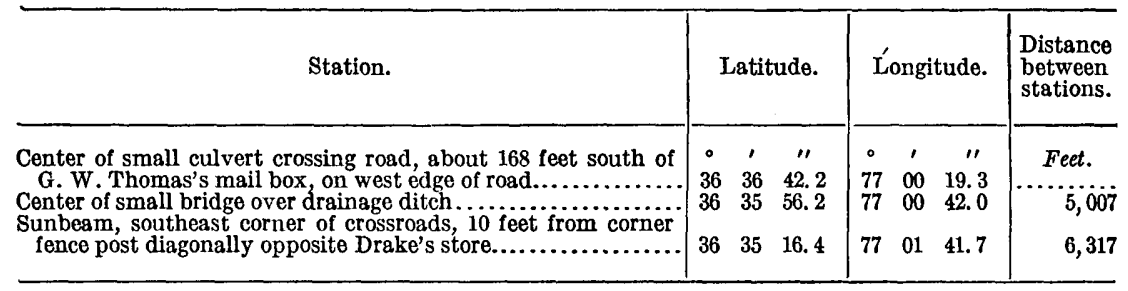

South along highways near east border of quadrangle.

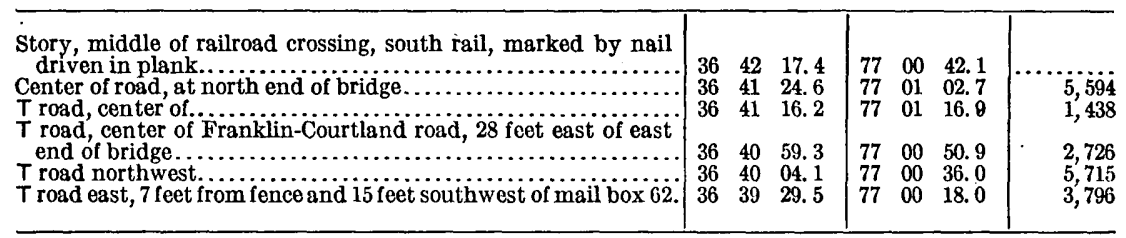

\section{North along highways and railway.}

Southwest angle of triangle where three roads meet, near $\mathrm{C}$. Holland's house..............................................

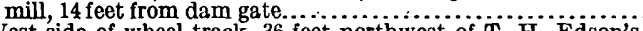
West side of wheel track, 36 feet northwest of $\mathbf{T}$. H. Edson's

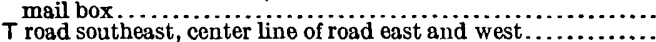

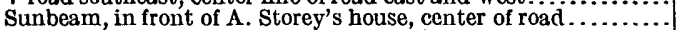

Sunbeam, a bout 1,000 feet northwest of school, crossroads at store. Sunbeam, 90 feet southeast of church door, T road southwest...

Center of small culvert, 382 feet south of R. B. Gray's house...

Handsom, 2 miles west of, in front of church, center of crossroads

$T$ road west, about 8 feet from a point 10 feet south of $J$. T.

Barrett's mail box................................................. Road crossing, over fourth cross tie from first rail joint west of

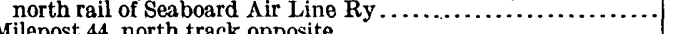

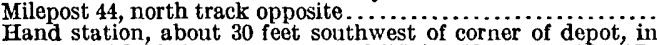
corner of field; iron post stamped "Prim. Trav. Sta. No.9P, 1918

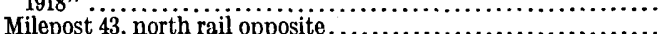

Delaware, 0.5 mile west of, west end of second bridge west of,

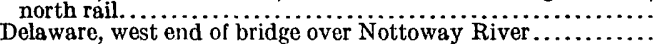

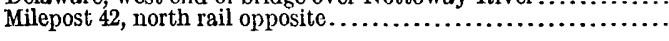

\begin{tabular}{rrr|rrr|r}
36 & 33 & 07.4 & 77 & 01 & 22.7 & $\ldots \ldots \ldots$ \\
36 & 33 & 11.7 & 77 & 01 & 08.2 & 1,262 \\
& & & & & & \\
36 & 33 & 53.2 & 77 & 01 & 08.7 & 4,192 \\
36 & 34 & 38.9 & 77 & 01 & 15.2 & 4,655 \\
36 & 34 & 53.3 & 77 & 01 & 22.9 & 1,587 \\
36 & 35 & 16.6 & 77 & 01 & 42.0 & 2,823 \\
36 & 35 & 36.5 & 77 & 01 & 58.2 & 2,408 \\
36 & 36 & 36.0 & 77 & 02 & 36.1 & 6,771 \\
36 & 37 & 00.0 & 77 & 02 & 46.0 & 2,550 \\
36 & 37 & 57.7 & 77 & 02 & 59.6 & 5,946 \\
& & & & & \\
36 & 38 & 29.4 & 77 & 03 & 11.2 & 3,338 \\
36 & 38 & 32.3 & 77 & 02 & 12.0 & 4,831 \\
& & & & & & \\
36 & 38 & 32.7 & 77 & 01 & 50.5 & 1,753 \\
36 & 38 & 38.7 & 77 & 01 & 08.0 & 3,514 \\
36 & 38 & 43.6 & 77 & 00 & 51.4 & 1,443 \\
36 & 38 & 54.3 & 77 & 00 & 15.3 & 3,138 \\
36 & 38 & 56.7 & 77 & 00 & 07.3 & 697 \\
& & & & & \\
\hline
\end{tabular}

Magnetic declination near east border of quadrangle, $4.5^{\circ} \mathrm{W}$.

Southeast along Seaboard Air Line Ry. (By H. J. Switzer in 1918.) 
BOYKINS QUADRANGLE-Continued.

East along Southern Ry. In north part of quadrangle. (By J. J. Phelan in 1917.)

Station.

\begin{tabular}{|c|c|c|c|c|c|c|}
\hline \multicolumn{3}{|c|}{ Latitude. } & \multicolumn{3}{|c|}{ Longitude. } & $\begin{array}{c}\begin{array}{c}\text { Distance } \\
\text { between } \\
\text { stations. }\end{array} \\
\text { Feet. }\end{array}$ \\
\hline $\begin{array}{l}\circ \\
36 \\
36 \\
36 \\
36 \\
36 \\
36 \\
36 \\
36 \\
36 \\
36 \\
36\end{array}$ & $\begin{array}{l}\prime \\
43 \\
43 \\
42 \\
42 \\
42 \\
42 \\
42 \\
42 \\
43 \\
43 \\
43\end{array}$ & $\begin{array}{l}\prime \prime \\
05.7 \\
01.7 \\
59.1 \\
53.4 \\
45.0 \\
39.9 \\
40.5 \\
49.4 \\
11.8 \\
10.2 \\
04.7\end{array}$ & $\begin{array}{l}0 \\
77 \\
77 \\
77 \\
77 \\
77 \\
77 \\
77 \\
77 \\
77 \\
77 \\
77\end{array}$ & $\begin{array}{l}1 \\
14 \\
14 \\
13 \\
13 \\
12 \\
12 \\
12 \\
11 \\
10 \\
10 \\
09\end{array}$ & $\begin{array}{c}\prime \prime \\
39.0 \\
12.2 \\
43.3 \\
21.8 \\
39.3 \\
19.8 \\
00.8 \\
16.4 \\
18.9 \\
02.0 \\
40.5\end{array}$ & $\begin{array}{r}\text { Feet. } \\
\ldots .3,222 \\
2,363 \\
1,842 \\
3,566 \\
1,672 \\
1,548 \\
3,726 \\
5,200 \\
1,386 \\
1,831\end{array}$ \\
\hline $\begin{array}{l}36 \\
36 \\
36 \\
36 \\
36 \\
36 \\
36 \\
36 \\
36 \\
36 \\
36\end{array}$ & $\begin{array}{l}42 \\
42 \\
42 \\
42 \\
42 \\
42 \\
42 \\
42 \\
42 \\
43 \\
43\end{array}$ & $\begin{array}{l}58.9 \\
55.7 \\
51.5 \\
53.4 \\
54.6 \\
56.7 \\
57.7 \\
58.5 \\
59.9 \\
00.4 \\
04.3\end{array}$ & $\begin{array}{l}\mathbf{7 7} \\
\mathbf{7 7} \\
\mathbf{7 7} \\
\mathbf{7 7} \\
\mathbf{7 7} \\
\mathbf{7 7} \\
\mathbf{7 7} \\
\mathbf{7 7} \\
\mathbf{7 7} \\
77 \\
\mathbf{7 7}\end{array}$ & $\begin{array}{l}09 \\
08 \\
08 \\
07 \\
07 \\
06 \\
06 \\
05 \\
04 \\
04 \\
04\end{array}$ & $\begin{array}{l}07.2 \\
47.3 \\
12.1 \\
25.5 \\
07.4 \\
30.8 \\
02.7 \\
39.4 \\
55.4 \\
39.9 \\
07.9\end{array}$ & $\begin{array}{l}2,777 \\
1,651 \\
2,896 \\
3,804 \\
1,471 \\
2,987 \\
2,293 \\
1,895 \\
3,589 \\
1,264 \\
2,633\end{array}$ \\
\hline $\begin{array}{l}36 \\
36 \\
36 \\
36 \\
36 \\
36\end{array}$ & $\begin{array}{l}43 \\
42 \\
42 \\
42 \\
42 \\
42\end{array}$ & $\begin{array}{l}05.1 \\
57.6 \\
49.7 \\
\mathbf{3 5 . 6} \\
\mathbf{1 8 . 8} \\
\mathbf{1 7 . 4}\end{array}$ & $\begin{array}{l}77 \\
77 \\
77 \\
77 \\
77 \\
77\end{array}$ & $\begin{array}{l}03 \\
03 \\
02 \\
01 \\
00 \\
00\end{array}$ & $\begin{array}{l}53.8 \\
23.6 \\
51.7 \\
55.2 \\
47.8 \\
42.1\end{array}$ & $\begin{array}{r}1,148 \\
2,568 \\
2,725 \\
4,812 \\
5,748 \\
482\end{array}$ \\
\hline & 42 & 17.3 & 77 & 00 & 42.4 & 28 \\
\hline
\end{tabular}

West end of bridge 56.2

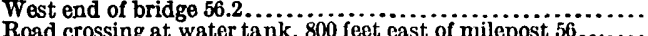

West end of bridge, south rail.................................

Milepost 55, north rail.

Capron 0.5 mile west of at west end of bridge south rail

Milepost 54 , south rail.

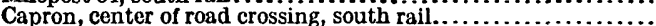

Milepost 53, south rail

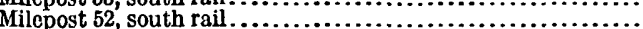

Pope, road crossing, south rail at center line

Pope, 0.875 mileast 0 nor 730 iect east of milepost 51 r rosd crossi

on main county road 730 cect east of millepost bl, road crossing

on main county road..........

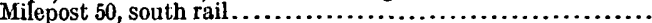

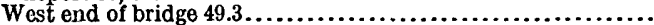

Milepost 49 , south rail

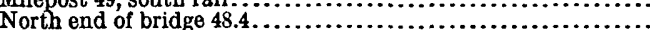

Milopost 48, south rail.

West end of bridge 47.6 .

Road crossing, 200 feet east of milepost 47 .................

West end of long bridge over Nottoway River into Courtland.

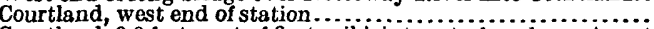

Courtland, 3.3 feet west of first rail joint east of road crossing at

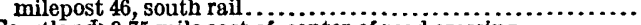

Courtland; 0.75 mile east of, center of road crossing............

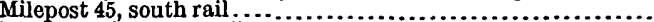

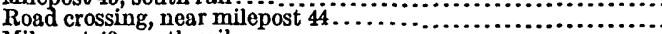

Milepost 43, south rail

Story, road crossing.......

Story, southwest corner of road crossing and Southern $R y$

crossing, near corner of wire fence; iron post stamped "Prim.

Trav. Sta. No. 11P, 1918"

\section{Southwest along highways in nor theast corner of quadrangle.}

$T$ road northeast near K. Sykes's mail box 37 , outer edge of road southeast and center line of $T$ road .........................

W. T. Davis's Grocery" west of tree with sign "2 miles to

Center of road, opposite Patton's paint sign “ “i milo to co court-

land"............

Courtland, road crossing....................................

\begin{tabular}{|rrr|rrr|r}
36 & 44 & 12.6 & 77 & 02 & 11.4 & $\ldots \ldots \ldots .$. \\
36 & 44 & 17.9 & 77 & 02 & 22.5 & $.1,050$ \\
36 & 43 & 28.9 & 77 & 03 & 16.9 & 6,642 \\
36 & 43 & 25.2 & 77 & 03 & 22.9 & 612 \\
36 & 43 & 05.1 & 77 & 03 & 53.8 & 3,235 \\
\hline
\end{tabular}

Mean magnetic declination for southeast corner of quadrangle, $4.1^{\circ} \mathrm{W}$.

South near west border of quadrangle. (By J. J. Phelan in 1918.)

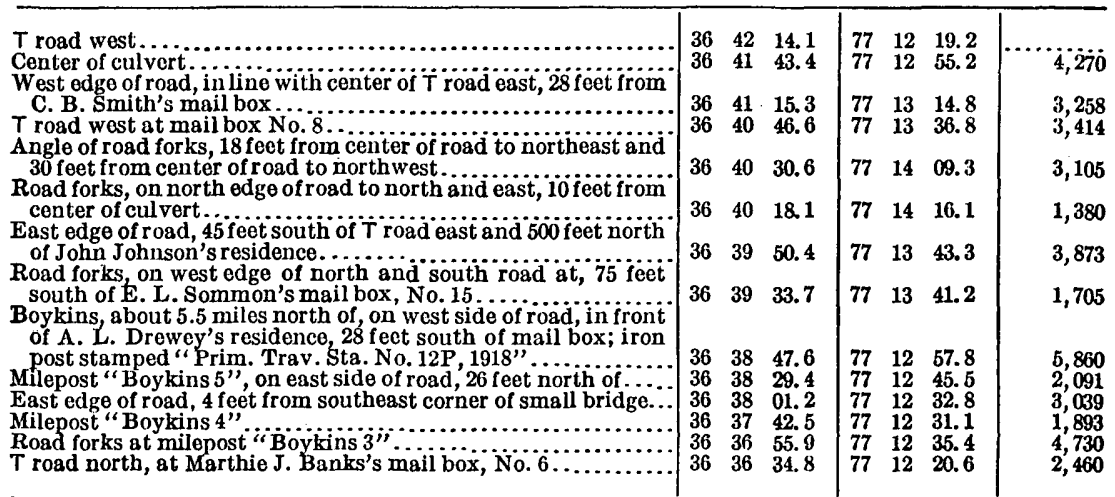


BOTKINS QUADRANGLE-Continued.

East through center of quadrangle along Seaboard Air Line Ry.

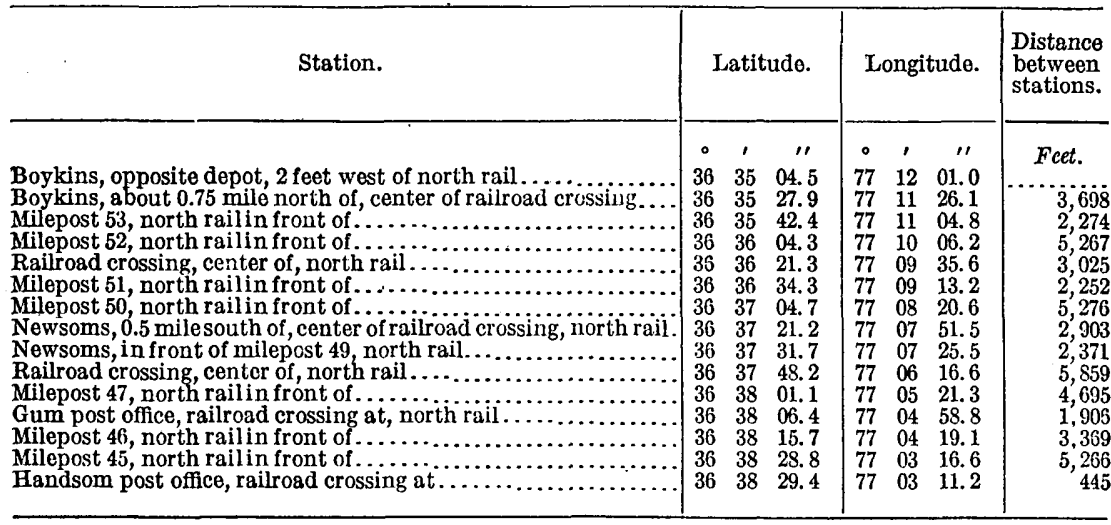

From Newsoms northwest and southwest along highways to Boykins.

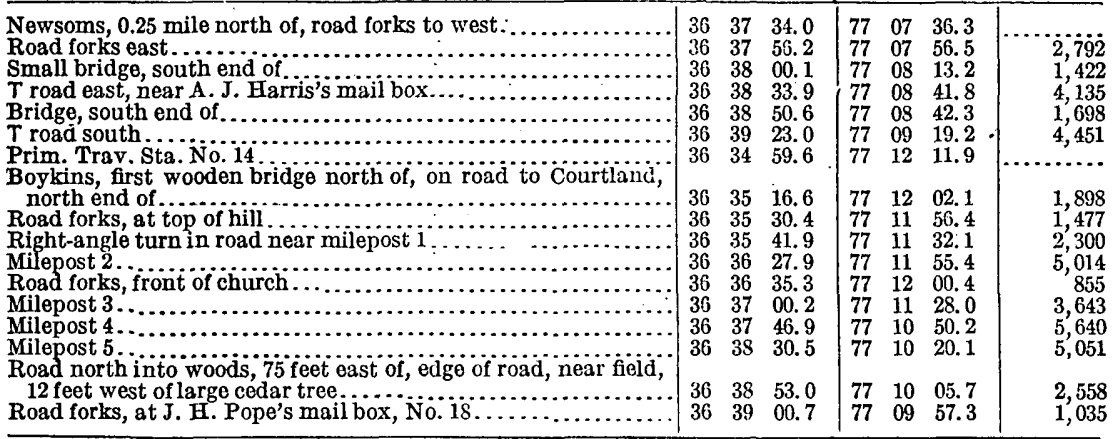

Magnetic declination for south border of quadrangle, $4^{\circ} 14^{\prime} \mathrm{W}$; f for west border of quadrangle, $4^{\circ} 02^{\prime} \mathrm{W}$; for center of quadrangle, $4^{\circ} 16^{\prime} \mathrm{W}$.

CAMP ABRAHAM EUSTIS.

For positions see Yorktown quadrangle, pages 499 to 501 . CAMP HUMPHREYS, INCLUDING PARTS OF FAIRFAX, INDIAN HEAD, OCCOQUAN, From Mount Vernon flag pole northeast to Dunn triangulation station. (By School of Topography, U. S. Army, in 1918).

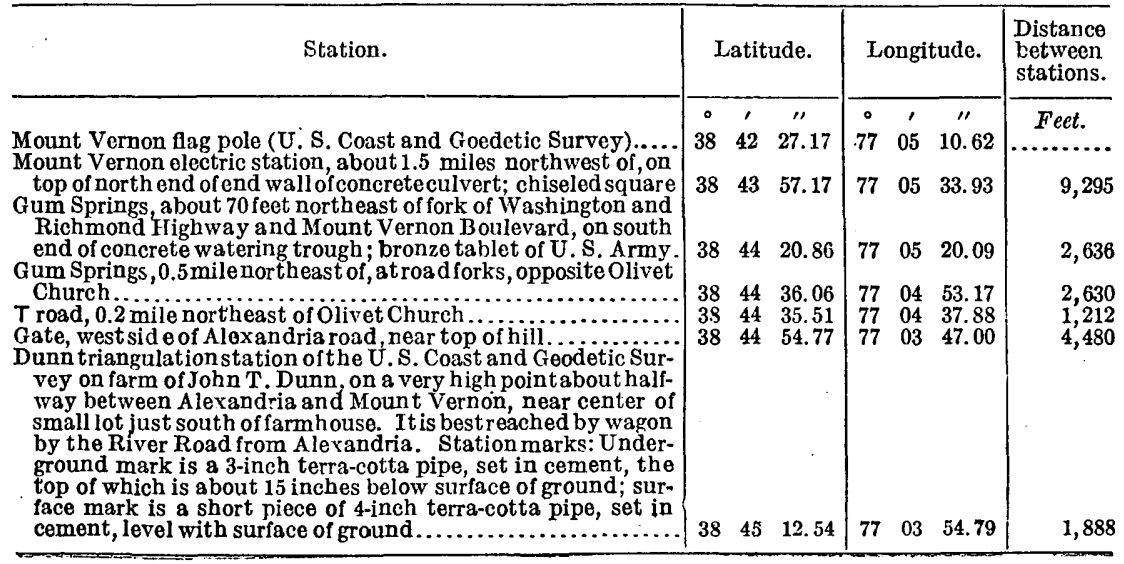


CAMP HUMPHREYS, INCLUDING PARTS OF FAIRFAX, INDIAN HEAD, OCCOQUAN, AND WASHINGTON QUADRANGLES-Continued.

From Mount Vernon flag pole to Gunston Hall.

\begin{tabular}{|c|c|c|c|c|c|c|c|}
\hline Station. & \multicolumn{3}{|c|}{ Latitude. } & \multicolumn{3}{|c|}{ Longitude. } & $\begin{array}{l}\text { Distance } \\
\text { between } \\
\text { stations. }\end{array}$ \\
\hline Mount Vernon flag pole (U. S. Coast and Geodetic Survey sta- & $\therefore$ & ' & " & $\therefore$ & & $"$ & Feet. \\
\hline Mount vernon station, 0.1 milc northwest of, center of concrete & & 42 & 27.17 & 77 & 05 & 10.64 & \\
\hline $\begin{array}{l}\text { bridge carrying electric railroad over highway......................... } \\
\text { Mount Vernon station, } 0.6 \text { mile northwest of, crossroads, at }\end{array}$ & & 42 & 40.74 & & 05 & 15.83 & 1,438 \\
\hline northwest corner of concrete culvert; chiseled square......... & 38 & & 01.29 & 77 & 05 & 46.63 & 3,208 \\
\hline T road opposite west gate to Mount Vernon estate........ & & & 46.94 & 77 & 05 & 48.67 & 1,460 \\
\hline $\begin{array}{l}\text { Troad south } \\
\text { Road forks, } 0.3 \text { mile south of Washington and Richmond High. }\end{array}$ & & 42 & 57.85 & & 06 & 33.82 & 3,747 \\
\hline 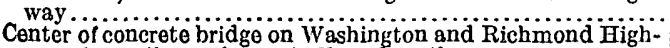 & & 43 & 05.75 & 77 & 07 & 06.22 & 2,690 \\
\hline 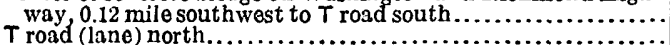 & 38 & & 19.69 & 77 & 07 & 20.00 & 1,784 \\
\hline 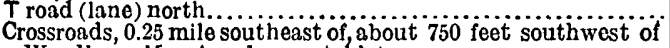 & & 43 & 13.40 & 77 & 07 & 32.83 & 1,201 \\
\hline 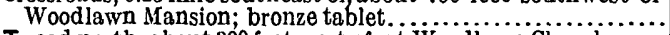 & 38 & & 52.79 & 77 & 08 & 20.77 & \\
\hline $\begin{array}{l}\text { T road north, about } 300 \text { feet west of, at Woodlawn Church..... } \\
\text { Intersection of Washington and Richmond Highway and }\end{array}$ & & & 48.51 & 77 & 08 & 27.56 & \\
\hline $\begin{array}{l}\text { cement road leading into Camp Humphreys... } \\
\text { Trestle 5, Camp A. A. Humphreys } R \text {. } R \text {., center of road } \ldots \ldots \ldots\end{array}$ & $\begin{array}{l}38 \\
38\end{array}$ & 42 & 40.52 & 77 & 08 & 37.10 & 1,107 \\
\hline Accotink village, at $T$ roads, east end of south wall of concrete & & & 31.80 & 77 & 09 & 19.32 & 3,461 \\
\hline $\begin{array}{l}\text { culvert; bronze tablet of U. S. Army ............ } \\
\text { Accotink Creek, east end of steel bridge over, on washington }\end{array}$ & 38 & 42 & 30.93 & 77 & 09 & 33.79 & 1,151 \\
\hline 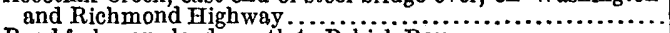 & 38 & & 17.69 & 77 & 09 & 57.27 & 2,296 \\
\hline $\begin{array}{l}\text { Road forks, oneleads south to Pohick Bay ............................. } \\
\text { Fork of Washington and Rich mond Highwy and Lewis Chapel }\end{array}$ & 38 & 41 & 54.31 & 77 & 10 & 16.16 & 2,799 \\
\hline $\begin{array}{l}\text { road, about } 1 \text { mile southeast of Pohick Church......................... } \\
\text { Crossroads, } 0.6 \text { mile south of Pohick Church, near rifle range }\end{array}$ & & 42 & 03.11 & 77 & 10 & 46.03 & 2,532 \\
\hline 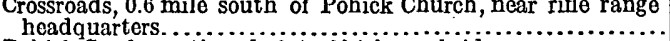 & 38 & 42 & 05.61 & 77 & 11 & 49.40 & 5,033 \\
\hline Pohick Creek, south end of steel highway bridge over.............. & 38 & 41 & 53.34 & 77 & 12 & 05.89 & 1,804 \\
\hline $\begin{array}{l}\text { Road fork, } 0.5 \text { mile southwest of Pohick Creek highway bridge.. } \\
\text { Lewis Chapel crossroads, about } 50 \text { feet southwest of, on concrete }\end{array}$ & 38 & 41 & 33.01 & 77 & 12 & 21.17 & 2,388 \\
\hline well platform; bronze tablet of U. S. Army .................. & 38 & 3 & 13.94 & 77 & 12 & 31.73 & 2,103 \\
\hline Road forks, 0.3 mile south of Lewis Chapel.... & 38 & 40 & 58.02 & 77 & 12 & 32.11 & 1,611 \\
\hline Lebanon School, center of roas & 38 & 40 & 41.65 & 77 & 12 & 09.93 & 2,415 \\
\hline T road northeast (private road) $\ldots \ldots \ldots \ldots \ldots$ & 38 & 40 & 20.21 & 77 & 11 & 34.84 & 3,528 \\
\hline Road forks, 1 mile southeast of Lebanon School... & 38 & 40 & 07.43 & 77 & 11 & 13.97 & 2,099 \\
\hline 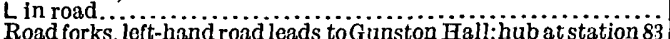 & 38 & 40 & 17.65 & 77 & 10 & 49.60 & 2,102 \\
\hline $\begin{array}{l}\text { Road forks, loft-hand road leads to Gunston Hall; hub at station } 83 \\
\text { Road fork, } 0.2 \text { mile northwest of Gunston Hall, on north end of }\end{array}$ & 38 & 40 & 04.67 & 77 & 10 & 11.21 & 3,316 \\
\hline $\begin{array}{l}\text { concrete culvert; chiseled square } \ldots \ldots \ldots \ldots \ldots \ldots \ldots \\
\text { Gunston }\end{array}$ & 38 & 39 & 57.89 & 77 & 09 & 47.90 & 1,971 \\
\hline 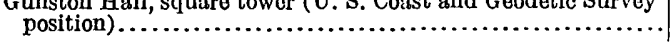 & 38 & & 50.60 & 77 & 09 & 37.52 & 1,105 \\
\hline
\end{tabular}

NoтE.-The tower on Gunston Fall has been removed; point tied to was the approximate conter of house.

From point 1 mile southeast of Pohick Church via Washington and Richmond Highway and Richmond, Fredericksburg \& Potomac R. R. to Accotink station.

Fork of Washington and Richmond Highway and Lowis Chapel road, about 1 mile southeast of Pohick Church................ Pohick Church, about 200 feet east of; 25 feet north of intersection of crossroads, in edge of field; conerete post, bronze tablet. .

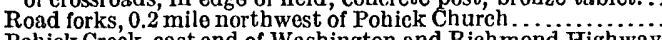

Pohick Creek, east end of Washington and Richmond Highway

Lorton station, about 600 feet north of, in east sido of south abutment of Richmond, Fredericksburg \& Potomac R. R. bridge over Washington and Richmond Highway; bronze tablet of U. S. Army.

Pohick Creek, about io feet from south end of Richmond, Fredericksburg \& Potomac R. R. bridge, on center line of south-

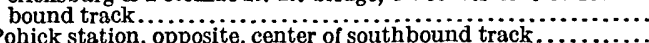

Pohick station, opposite, center of south bound track .........

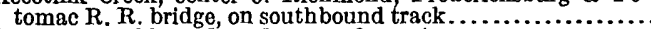

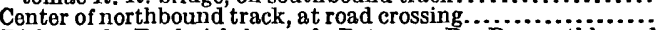

Richmond, Fredericksburg \& Potomac R. R. northbound track, opposite tangent on Camp A. A. Humphreys $\mathbf{R}$. R ....

Accotink station, opposite operator's window, center of north-

bound track............ 300 feet north of, south end of $\mathrm{Rich}$

mond, Fredericksburg \& Potomac R. R. trestle; bronze

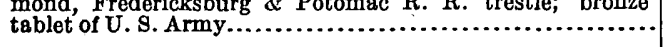

\begin{tabular}{|rrr|rrr|r}
38 & 42 & 03.11 & 77 & 10 & 46.03 & $\ldots \ldots \ldots$ \\
38 & 42 & 33.02 & 77 & 11 & 36.06 & $\ldots \ldots \ldots$. \\
38 & 42 & 36.25 & 77 & 11 & 50.36 & 1,183 \\
38 & 42 & 12.96 & 77 & 12 & 51.23 & 5,369 \\
& & & & & & \\
38 & 42 & 20.04 & 77 & 13 & 16.44 & 2,120 \\
38 & 42 & 45.58 & 77 & 13 & 04.07 & 2,750 \\
38 & 43 & 0985 & 77 & 12 & 35.96 & 3,314 \\
38 & 43 & 22.42 & 77 & 12 & 15.72 & 2,047 \\
38 & 43 & 37.77 & 77 & 11 & 36.16 & 3,500 \\
38 & 44 & 06.12 & 77 & 11 & 12.90 & 3,408 \\
38 & 44 & 16.95 & 77 & 11 & 09.81 & 1,120 \\
& & & & & & \\
38 & 44 & 19.46 & 77 & 11 & 09.00 & 260 \\
& & & & & \\
\end{tabular}


CAMP HUMPHREYS, INCLUDING PARTS OF FAIRFAX, INDIAN FEAD, OCCOQUAN, AND WASHINGTON QUADRANGLES-Continued.

From Pohick Church via highway to Potters Hill crossroads.

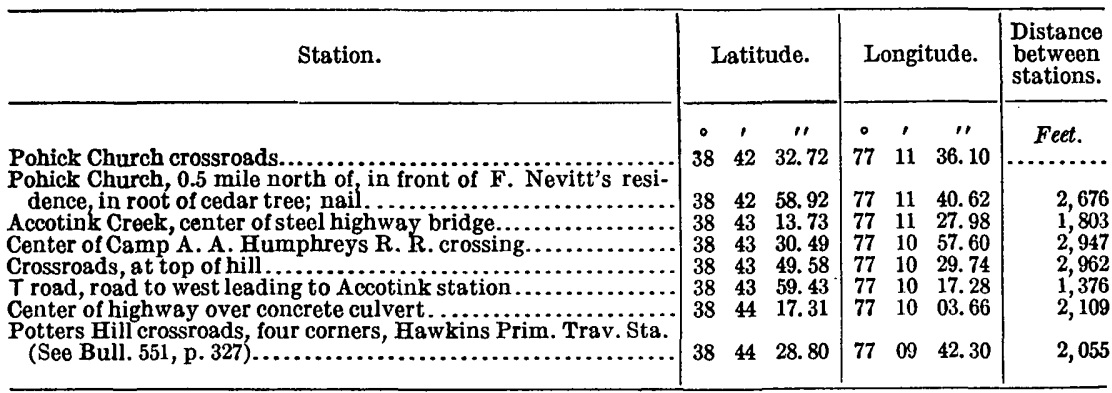

From Potters Bull crossroads to Accotink Village.

\begin{tabular}{|c|c|c|c|c|c|c|c|}
\hline 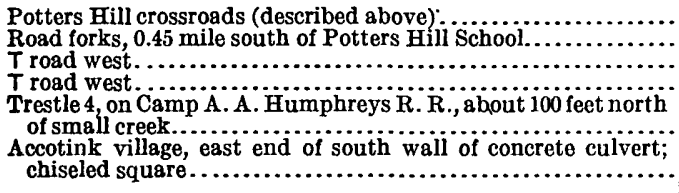 & $\begin{array}{l}38 \\
38 \\
38 \\
38 \\
38 \\
38\end{array}$ & $\begin{array}{l}44 \\
44 \\
43 \\
43 \\
42\end{array}$ & $\begin{array}{l}28.80 \\
07.88 \\
48.50 \\
18.86 \\
49.56 \\
30.93\end{array}$ & $\begin{array}{l}77 \\
77 \\
77 \\
77\end{array}$ & $\begin{array}{l}09 \\
09 \\
09 \\
09\end{array}$ & $\begin{array}{l}42.30 \\
39.00 \\
40.68 \\
35.59 \\
35.06 \\
33.79\end{array}$ & $\begin{array}{r}2,136 \\
1,962 \\
3,023 \\
2,971\end{array}$ \\
\hline
\end{tabular}

From Potters Hill crossroads via Woodlawn Road to Washington and Richmond highway.

\begin{tabular}{|c|c|c|c|c|c|c|c|}
\hline 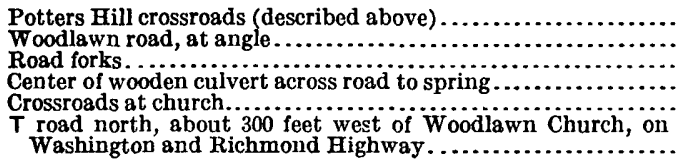 & $\begin{array}{l}38 \\
38 \\
38 \\
38 \\
38\end{array}$ & $\begin{array}{l}44 \\
43 \\
43 \\
43 \\
43 \\
42\end{array}$ & $\begin{array}{l}28.80 \\
56.72 \\
48.31 \\
30.49 \\
12.60 \\
48.51\end{array}$ & $\begin{array}{l}77 \\
77 \\
77 \\
77 \\
77 \\
77\end{array}$ & $\begin{array}{l}09 \\
09 \\
09 \\
08 \\
08 \\
08\end{array}$ & $\begin{array}{l}42.30 \\
21.53 \\
02.24 \\
50.03 \\
40.47 \\
\\
27.56\end{array}$ & $\begin{array}{l}657 \\
755 \\
052 \\
967\end{array}$ \\
\hline
\end{tabular}

From Washington and Richmond Highway south along Belvoir Peninsula to old Fairfax Landing.

$T$ road north, about 300 feet west of Woodlawn Church, on

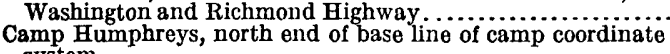

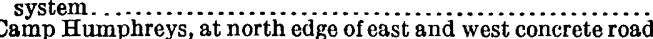
along north side of north section; iron bolt..................

Camp Humphreys, steel water tower, in concrete base of feed pipe; bronze tablet (Spur line. This point is unchecked.)... Camp Humphreys, at north edge of east and west concrete road along north side of north section; iron bolt................. Camp Humphreys, north edge of east and west road along south

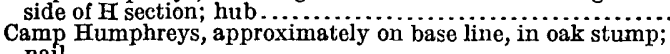

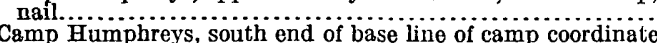

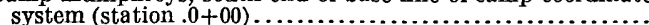
ntersection of road to Belvoir Dock and road to upper (new) Forks of road to Belvoir Dock and old road to old wateh house,

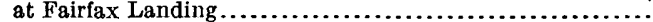
White house, the farther south of two, chimney of $(\mathrm{U} . \mathrm{S} . \mathrm{C}$. \&

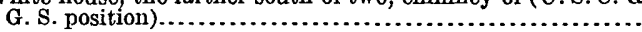

\begin{tabular}{|rrr|rrr|r}
38 & 42 & 48.51 & 77 & 08 & 27.56 & $\ldots \ldots \ldots$ \\
38 & 42 & 37.25 & 77 & 08 & 50.66 & $\ldots \ldots \ldots$ \\
38 & 41 & 37.01 & 77 & 08 & 19.51 & 6,577 \\
38 & 41 & 35.18 & 77 & 08 & 30.01 & 853 \\
38 & 41 & 37.01 & 77 & 08 & 19.51 & 853 \\
38 & 41 & 09.49 & 77 & 08 & 05.34 & 3,005 \\
38 & 40 & 51.34 & 77 & 07 & 55.73 & 1,990 \\
38 & 40 & 37.90 & 77 & 07 & 48.88 & 1,466 \\
38 & 40 & 53.07 & 77 & 07 & 46.10 & 1,549 \\
38 & 41 & 03.30 & 77 & 07 & 49.57 & 1,069 \\
38 & 41 & 00.86 & 77 & 07 & 28.34 & 1,703 \\
\hline
\end{tabular}

577

853

3,005

466

549

069

From Accotink station along highways southeast to Accotink village.

Richmond, Fredericksburg \& Potomac Ry. track, 1,120 feet south of Accotink station, opposite tangent on Camp A. A.

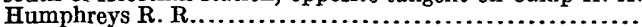

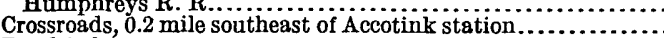
Top of hill, west side of road, in stump; nail, painted " $\mathrm{P} . \mathrm{T}$.

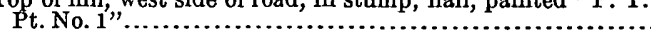
Camp A. A. Humphreys $\mathrm{R}$. R., 100 feet southeast of crossing, in front of $W . T$. Jones's residence, in root of oak tree, painted "U. S. A. 71.3 B. M.;" copper nail.

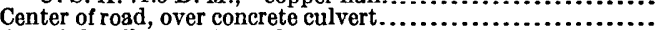

Accotink village, at $T$ roads, east end of south wall of concrete culvert; chiseled square..................................

\begin{tabular}{|rrr|rrr|r} 
& & & & & & \\
38 & 44 & 06.12 & 77 & 11 & 12.90 & $\ldots \ldots \ldots$ \\
38 & 44 & 04.69 & 77 & 11 & 03.58 & 750 \\
38 & 44 & 00.10 & 77 & 10 & 30.89 & 2,630 \\
38 & 43 & 35.22 & 77 & 10 & 29.63 & 2,515 \\
& & & & & & \\
38 & 43 & 12.30 & 77 & 10 & 13.59 & 2,641 \\
38 & 42 & 46.57 & 77 & 09 & 36.94 & 3,896 \\
38 & 42 & 30.93 & 77 & 09 & 33.79 & 1,599 \\
\hline
\end{tabular}


CAMP HUMPHREYS, INCLUDING PARTS OF FAIRFAX, INDIAN HEAD, OCCOQUAN, AND WASHINGTON QUADRANGLES-Continued.

From J. S. Geological Survey point Hawkins near Accotink station, on Southern Ry., to Junction of telegraph and gravel roads. (By School of Topography, U. S. Army, in 19i8.)

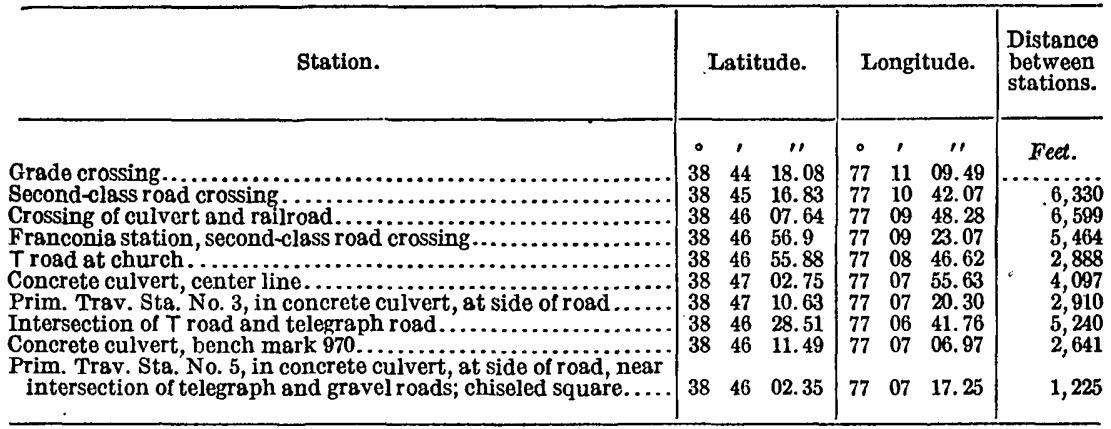

From Potters Hill crossroads, U. S. Geological Survey point Hawkins, to Junction of telegraph and gravel roads.

Four corners, 100 feet southeast to schoolhouse, 220 feet west to house (U. S. G. S. point Bull. 551, p. 327) ............

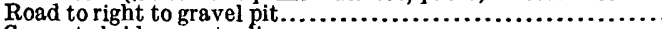

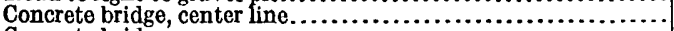

Concrete bridge.....................................................

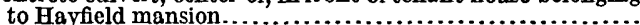

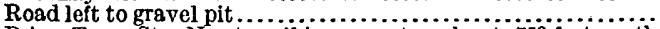

Prim. Trav. Sta. No. 4, nail in concrete culvert, 750 feet north of Hayfield mansion . ......................................

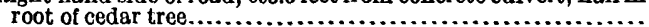

Wood culvert

\begin{tabular}{rll|lll|r}
38 & 44 & 28.8 & 77 & 09 & 42.3 & $\ldots$ \\
38 & 44 & 33.63 & 77 & 09 & 27.77 & \\
38 & 44 & 38.37 & 77 & 09 & 02.67 & 1,250 \\
38 & 44 & 53.67 & 77 & 08 & 37.40 & 2,047 \\
& & & & & & 2,529 \\
38 & 45 & 03.48 & 77 & 08 & 27.10 & 1,285 \\
38 & 45 & 13.72 & 77 & 08 & 18.95 & 1,221 \\
38 & 45 & 23.34 & 77 & 08 & 14.34 & 1,040 \\
38 & 45 & 29.13 & 77 & 08 & 09.64 & 693 \\
38 & 45 & 42.68 & 77 & 07 & 53.58 & 1,871 \\
\hline
\end{tabular}

From intersection of telegraph and gravel roads (Prim. Trav. Sta. No. 5) to Gum Springs.

Prim. Trav. Sta. No. 6, 6 feet from oak tree which is bench mark 48.9...

Wooden bridge.

Prim. Trav. Sta. No. 7 , chiseled square on concrete culvert, at

$T$ road west, 1.2 miles from Gum Springs Church ..............

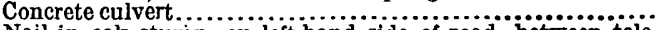

Nail in oak stump, on left-hand side of road, between telegraph posts 188 and 189,30 feet from $189 \ldots \ldots \ldots \ldots$

Gum Springs, at junccion of concrete road and Richmond and

Washington Highway, near colored church; nail.............

Gum Springs, Prim. Trav. Sta. No. 2, 70 feet northeast of road forks.

\begin{tabular}{rrr|rrr|r}
38 & 45 & 41.48 & 77 & 06 & 23.94 & 4,718 \\
38 & 45 & 37.41 & 77 & 05 & 46.08 & 3,028 \\
38 & 45 & 33.36 & 77 & 05 & 08.62 & 2,993 \\
38 & 45 & 04.76 & 77 & 05 & 04.70 & 2,907 \\
38 & 44 & 40.62 & 77 & 04 & 50.80 & $2,67:$ \\
38 & 44 & 36.47 & 77 & 04 & 52.95 & 448 \\
38 & 44 & 20.86 & 77 & 05 & 20.09 & 2,672
\end{tabular}

No magnetic declination.

From Mount Vernon to Hunter via Washington-Virginia Ry. (electric) and from Funter to Fort Hunt vla highway. (By H. S. Senseney in 1920.)

Mount Vernon flag pole, U. S. Coast and Geodetic Survey

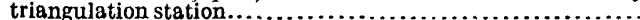

Second-class road crossing, 2 feet west of telephone pole. at northwest corner of crossing; copper nail in hub, marked

Miller, center of track, in front of station, copper nail in tie

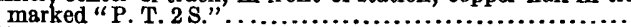

Riverside, center of track in front of station; copper nail in

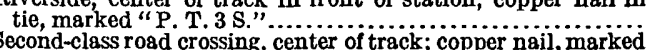
Second-class road crossing, center of track; copper nail, marked

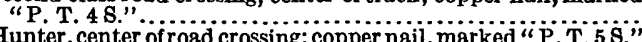

$T$ road west, in southwest corner, in root of cedar tree; copper

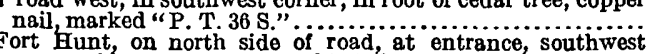

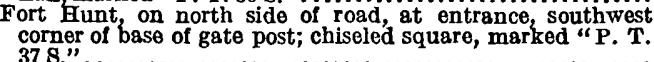

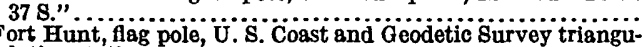

lation station pole, U. S. Coast and Geodetic Survey triangu-

\begin{tabular}{rrr|rrr|r}
38 & 42 & 27.17 & 77 & 05 & 10.64 & $\ldots \ldots \ldots$ \\
38 & 42 & 55.54 & 77 & 05 & 06.40 & 2,890 \\
38 & 43 & 02.59 & 77 & 04 & 40.73 & 2,155 \\
38 & 43 & 08.89 & 77 & 04 & 26.87 & 1,269 \\
38 & 43 & 12.31 & 77 & 04 & 19.33 & 691 \\
38 & 43 & 22.02 & 77 & 03 & 53.38 & 2,279 \\
38 & 43 & 19.11 & 77 & 03 & 39.81 & 1,115 \\
38 & 43 & 07.30 & 77 & 03 & 27.79 & 1,529 \\
38 & 42 & 56.46 & 77 & 03 & 06.59 & 2,006 \\
\hline
\end{tabular}


CAMP HUMPHREYS, INCLUDING PARTS OF FAIRFAX, INDIAN HEAD, OCCOQUAN, AND WASHINGTON QUADRANGLES-Continued.

From Hunter to New Alexandria via Washington-Virginia Ry. (electric).

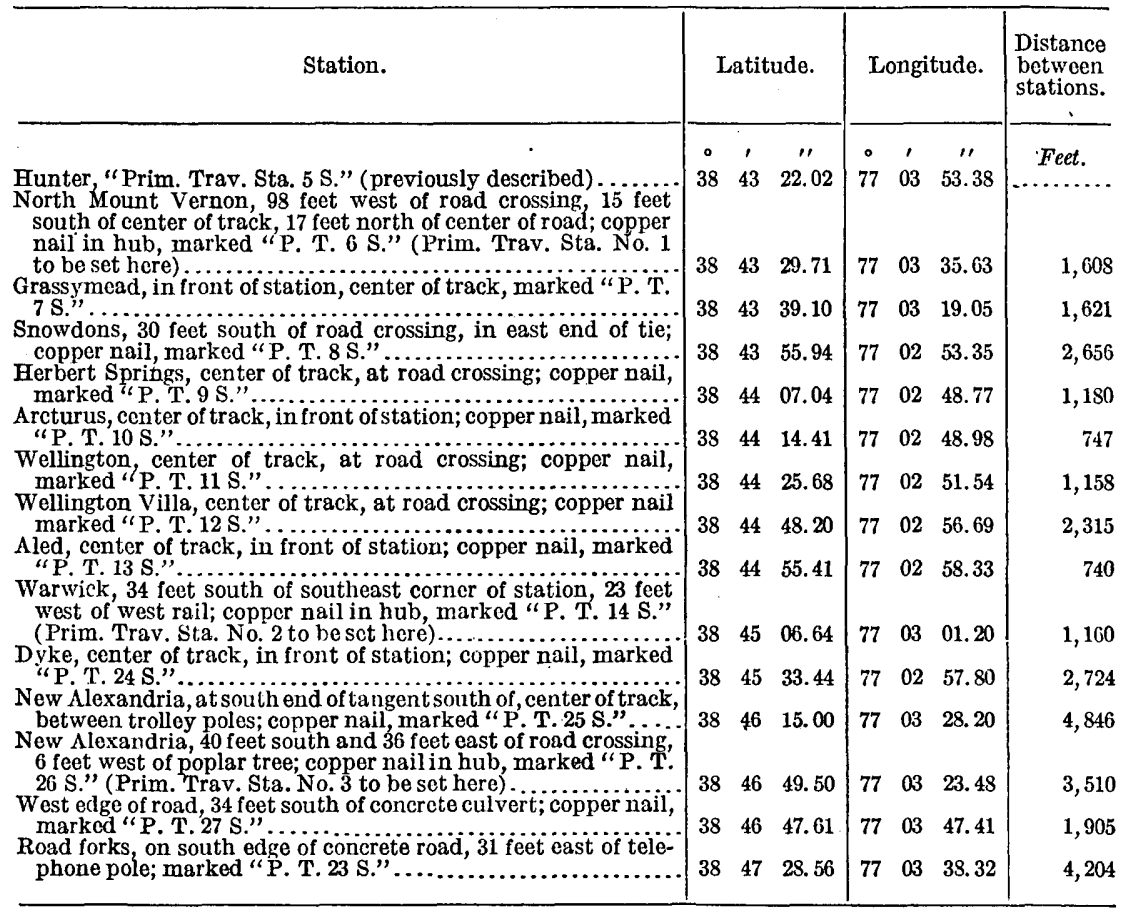

From Gum Springs to New Alezandria via highway.

Gum Springs, 70 feet northeast of fork of Washington and Richmond Highway and Mount Vernon Boulevard, south end of watering trough; chiseled square.

Gum Springs Church, 35 feet northeast of, in root of oak tree;

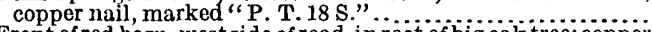

Front of red barn, west side of road, in root of big oak tree; copper

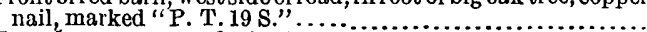

$T$ road west, east end of culvert, at northwest corner;

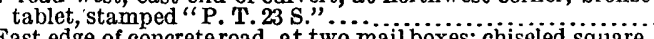

East edge of concrete road, at two mail boxes; chiseled square, marked " $P$ T $15 \mathrm{~S}$ "

marked "P. $\mathrm{T}$. 15 .

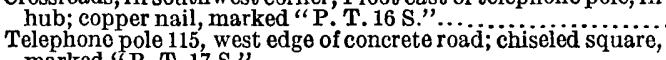

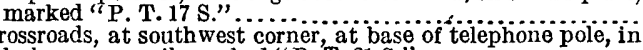

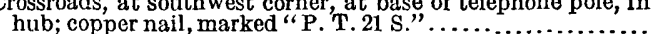

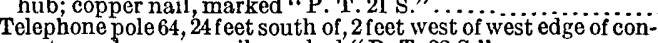

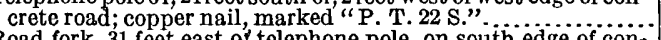

Road fork, 31 feet east of telephone pole, on south edge of con-

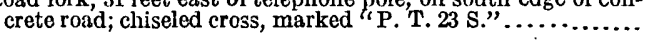

\begin{tabular}{rrr|rrr|r}
38 & 44 & 20.86 & 77 & 05 & 20.09 & $\ldots \ldots \ldots$ \\
38 & 44 & 37.50 & 77 & 04 & 52.58 & 2,753 \\
38 & 45 & 15.50 & 77 & 05 & 11.60 & 4,128 \\
38 & 45 & 33.22 & 77 & 05 & 08.60 & 1,808 \\
38 & 45 & 47.22 & 77 & 05 & 04.09 & 1,463 \\
38 & 45 & 57.08 & 77 & 05 & 03.22 & 998 \\
38 & 46 & 20.84 & 77 & 04 & 53.68 & 2,520 \\
38 & 46 & 52.66 & 77 & 04 & 45.42 & 3,284 \\
38 & 47 & 15.60 & 77 & 04 & 01.80 & 4,161 \\
38 & 47 & 28.56 & 77 & 03 & 38.32 & 2,275 \\
\hline
\end{tabular}

\section{From Gum Springs along highway to Funter.}

Prim. Trav. Sta. 18.

T road south, at southeast corner, 6 feet west of corner fence post,

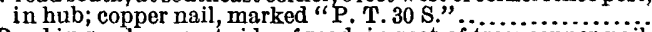

Bend in road, on east side of road, in root of tree; copper nail, marked "P. T. 31 S.".

$T$ road south, in southwest corner of, in root of smail oak tree;

copper nail, marked " $P$. T. $32 \mathrm{~S}$." Lane west to house, on east side of road, in root of pine tree; copper nail, marked " $\mathrm{P}$. $\mathrm{T}$. $33 \mathrm{~S}$. " ….... T road west, in southwest corner, in root of cedar tree; copper Road forks, on west side of road, in root of pine tree; copper nail

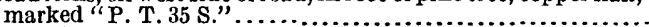

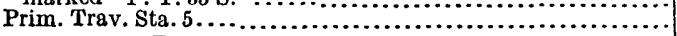

\begin{tabular}{|rrr|rrr|r}
38 & 44 & 37.50 & 77 & 04 & 52.58 & $\ldots \ldots \ldots$ \\
38 & 44 & 35.43 & 77 & 04 & 37.74 & 1,194 \\
38 & 44 & 15.74 & 77 & 04 & 23.70 & 2,282 \\
38 & 44 & 08.90 & 77 & 03 & 58.68 & 2,100 \\
38 & 43 & 59.71 & 77 & 04 & 00.22 & 939 \\
38 & 43 & 35.38 & 77 & 04 & 06.09 & 2,508 \\
38 & 43 & 25.26 & 77 & 04 & 08.25 & 1,040 \\
38 & 43 & 22.02 & 77 & 03 & 53.38 & 1,223
\end{tabular}


CAMP HUMPHREYS, INCLUDING PARTS OF FAIRFAX, INDIAN HEAD, OCCOQUAN, AND WASHINGTON QUADRANGLES-Continued.

Southeast along highway to Warwick.

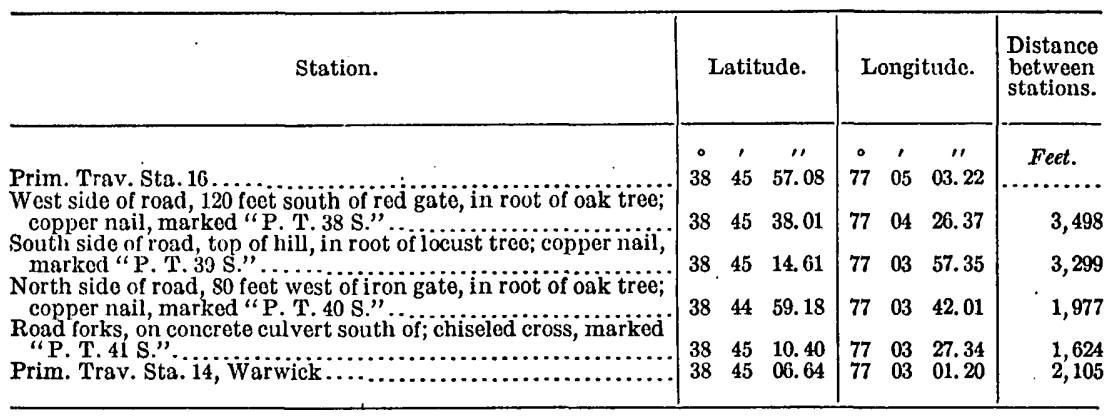

From Pohlck station north along highway to Barker's crossroads. (By School of Topography, U. S. Army, in 1918.)

Intersection of abandoned road and main road.

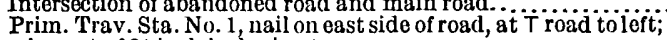

in root of 24 -inch jack pine tree........................

$T$ road west, in center of road $\ldots \ldots \ldots \ldots \ldots \ldots \ldots \ldots \ldots \ldots \ldots . . . \ldots \ldots$

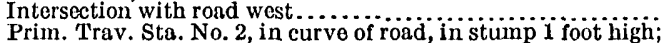

Prim. Trav. Sta. No. 2, in curve of road, in stump 1 foot high;

Barker's crossroads, near Davis's store, intersection of roads;

Prim. Trav. Sta. No. 3 .

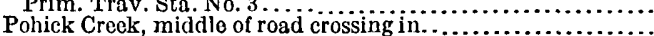

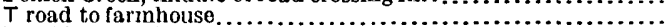

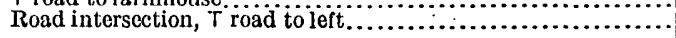

\begin{tabular}{|rrr|rrr|r}
38 & 43 & 25.40 & 77 & 12 & 34.95 & $\ldots \ldots \ldots$ \\
38 & 43 & 41.89 & 77 & 12 & 35.44 & 1,668 \\
38 & 44 & 18.50 & 77 & 12 & 41.22 & 3,733 \\
38 & 44 & 27.33 & 77 & 12 & 43.40 & 909 \\
38 & 44 & 54.24 & 77 & 12 & 44.87 & 2,725 \\
& & & & & & \\
38 & 45 & 34.08 & 77 & 13 & 04.80 & 4,328 \\
38 & 45 & 25.84 & 77 & 13 & 36.12 & 2,618 \\
38 & 45 & 23.86 & 77 & 13 & 48.23 & 980 \\
38 & 45 & 24.69 & 77 & 14 & 00.53 & 970 \\
& & & & & \\
\hline
\end{tabular}

Southeast along highway from road intersectlon at Gambol's store 1 mile south of Pohick School to Pohick station.

Second-class private road to left.

Middle Run, center

Prim. Trav. Sta. No. 1,50 feet east of intersection of inain road

and second-class road to Pohick School.

Small culvert.

Private road to left.

Second-class abandoned road to south west of blazed 10-inch black-0ak tree: hub painted "P.T, No.2" Prim. Trav. Sta. No. 3, west side of road, chiseled cross on concrete post, "DCR igl4" "painted "P. T. N. 3 "........

Intersection of second-class road to left.

Second-class road to right...........

Intersection of pulp-wood road to left, east side of road, in root of 14-inch white-oak tree: nail................................... Prim. Trav. Sta. No. 4, iron bridge over Pohick Creek, on north

Pohick station, road crossing, at end of bridge...................

\begin{tabular}{|rrr|rrr|r}
38 & 45 & 11.41 & 77 & 13 & 55.47 & 1,400 \\
38 & 45 & 01.29 & 77 & 14 & 02.40 & 1,162 \\
38 & 44 & 36.79 & 77 & 14 & 04.13 & 2,482 \\
38 & 44 & 28.74 & 77 & 13 & 56.16 & 1,030 \\
38 & 44 & 25.23 & 77 & 13 & 52.60 & 453 \\
38 & 44 & 14.77 & 77 & 13 & 43.26 & 1,292 \\
38 & 44 & 09.90 & 77 & 13 & 33.27 & 933 \\
38 & 43 & 54.79 & 77 & 13 & 18.29 & 1,937 \\
38 & 43 & 50.18 & 77 & 13 & 15.99 & 500 \\
38 & 43 & 24.81 & 77 & 13 & 04.70 & 2,719 \\
38 & 43 & 18.51 & 77 & 12 & 54.22 & 1,050 \\
38 & 43 & 12.03 & 77 & 12 & 43.14 & 1,096 \\
38 & 43 & 12.31 & 77 & 12 & 34.65 & 673 \\
\hline
\end{tabular}

Along highways from Pohick Church southeast to rifle range. (By School of Topography, $\mathrm{U} . \mathrm{s}$. Army, in 1918.)

Prim. Trav. Sta. No. 1, nail in saddle of twin willow trees, 50 feet east of barn, painted "P. T. No. 1 " .................... Nail in stake, 1 foot from trunk of 10 -inch walnut tree, in front of Mrs. Grace Merchant's house, near rifle range.............. trce, 1,800 feet west of brick house, on S. Shephard's farm.

Prim. Trav. Sta. No. 3 , nail in root of 9 -inch cedar tree, on left side of road, 1,000 feet northwest of $S$. Shepard's house, painted "P. T. No. 3 "

\begin{tabular}{|rrr|rrr|r}
38 & 42 & 03.06 & 77 & 11 & 47.51 & $\ldots \ldots$ \\
38 & 41 & 21.48 & 77 & 11 & 36.23 & 4,298 \\
38 & 41 & 14.90 & 77 & 10 & 39.11 & 4,579 \\
38 & 41 & 31.33 & 77 & 10 & 13.70 & 2,614 \\
\hline
\end{tabular}


CAMP HUMPHREYS, INCLUDING PARTS OF FAIRFAX, INDIAN HEAD, OCCOQUAN, AND WASHINGTON QUADRANGLES-Continued.

From Shiloh Church via Sycamore Point to Sandy Point.

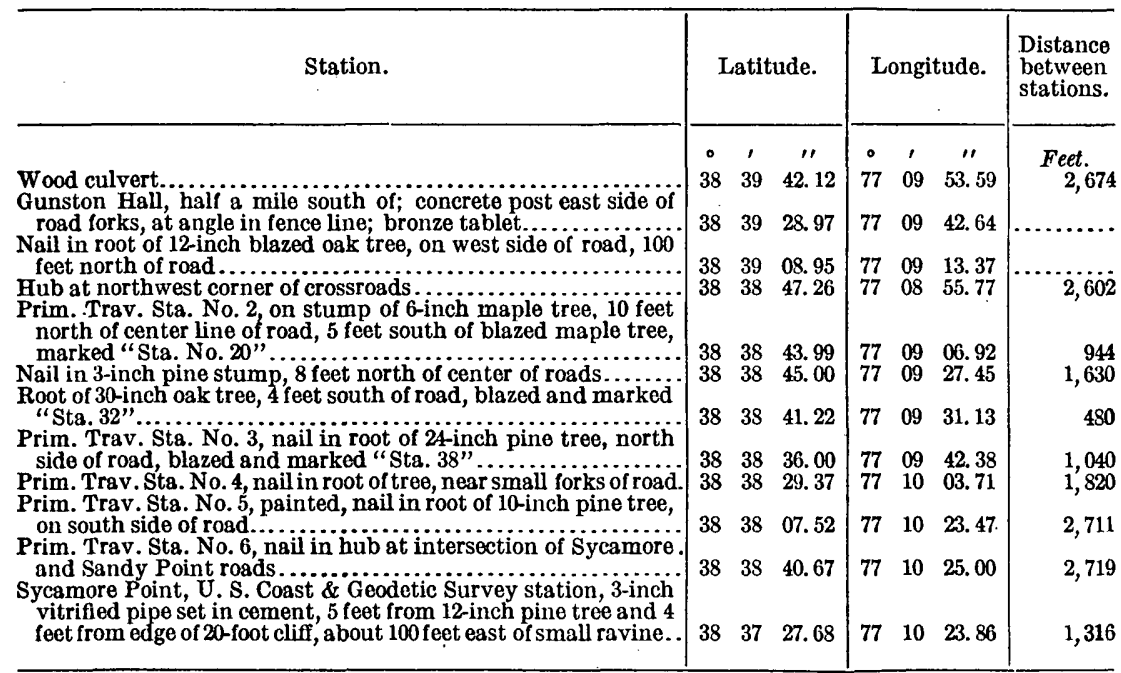

Near intersection of Sycamore Point and Sandy Point roads.

Nail in root of red-oak tree, on west side of road, at edge of woods,

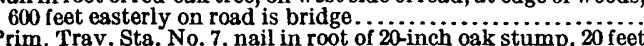
Prim. Trav. Sta. No. 7, nail in root of 20 -inch oak stump, 20 feet

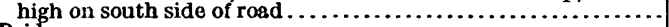

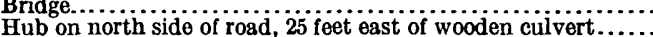

Sandy Point, Occoquan Bay, white flagpole (the stump only remains); U. S. Coast \& Geodetic Survey point..............

\begin{tabular}{|lll|lll|l}
38 & 37 & 45.01 & 77 & 11 & 11.58 & 3,722 \\
38 & 37 & 56.89 & 77 & 11 & 29.12 & 1,836 \\
38 & 37 & 59.37 & 77 & 11 & 43.51 & 1,167 \\
38 & 37 & 55.40 & 77 & 12 & 06.09 & 1,836 \\
38 & 37 & 57.11 & 77 & 12 & 46.84 & 3,240
\end{tabular}

From Primary Traverse Station 20 S to Primary Traverse Station 29 S. (By H. S. Senseney in 1920.)

Prim. Trav. Sta. No. $20 \mathrm{~S}$ (previously described) ............ \begin{tabular}{llll|lll}
38 & 45 & 33.22 & 77 & 05 & 08.60
\end{tabular} Crossroads, 19 feet west of, on south side of east and west road; copper nail in hub marked "P. T. $28 \mathrm{~S}$ " ...................

Small bridge, 100 feet south of, in south side of road, 7 feet east of tree; copper nail in hub marked "P.T. $29 \mathrm{~S}$ " ...............

\begin{tabular}{rrr|rrr|r}
38 & 45 & 33.22 & 77 & 05 & 08.60 & $\ldots \ldots \ldots$ \\
38 & 45 & 37.18 & 77 & 05 & 45.35 & 2,938 \\
38 & 45 & 06.80 & 77 & 06 & 04.55 & 3,430
\end{tabular}

From Primary Traverse Station 1 B to Primary Traverse Station 29 S.

$T$ road north, 1 foot south of concrete road; copper nail in hub, marked "P. T. P. No. 1 B" ........................... Gate post, 2 feet esst of, on north side of road; copper nail in hub, marked " $P$. T. $42 \mathrm{~S}$ "......................................... East and west wire fence, 13 feet north of, 14 feet east of end of north and south wire fence, on east edge of woods; copper nail

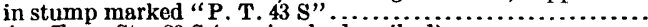

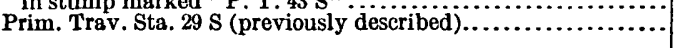

\begin{tabular}{|rrr|rrr|r}
38 & 44 & 07.51 & 77 & 05 & 46.12 & $\ldots \ldots \ldots$ \\
38 & 44 & 16.11 & 77 & 05 & 53.54 & 1,050 \\
& & & & & & \\
33 & 44 & 48.16 & 77 & 05 & 53.96 & 3,242 \\
38 & 45 & 06.80 & 77 & 06 & 04.55 & 2,064
\end{tabular}

From Gum Springs to old road forks near Doque Creek. (By D. S. Birkett in 1919.)

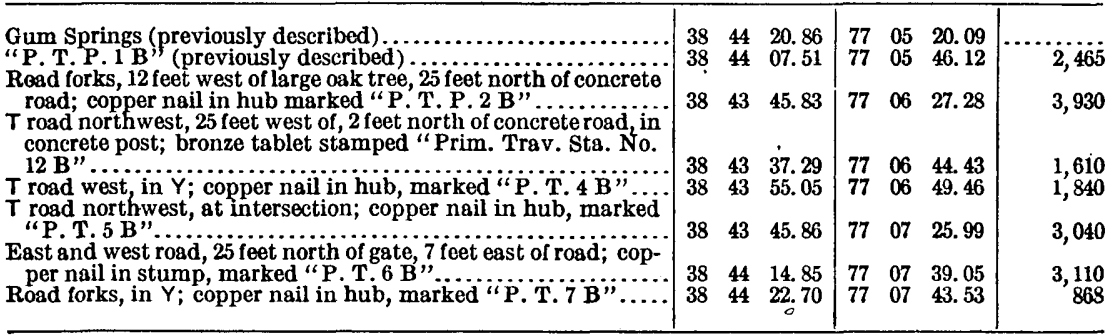

Magnetic declination, $5^{\circ} 35^{\prime} \mathrm{W}$. 

CAMP HUMPHREYS, INCIUDING PARTS OF FAIRFAX, INDIAN HEAD, OCCOQUAN,
AND WASHINGTON QUADRANGLES-Continued.

From old road forks near Dogue Creok to Potter's Hill crossroads. (By D. S. Birkett in 1920.)

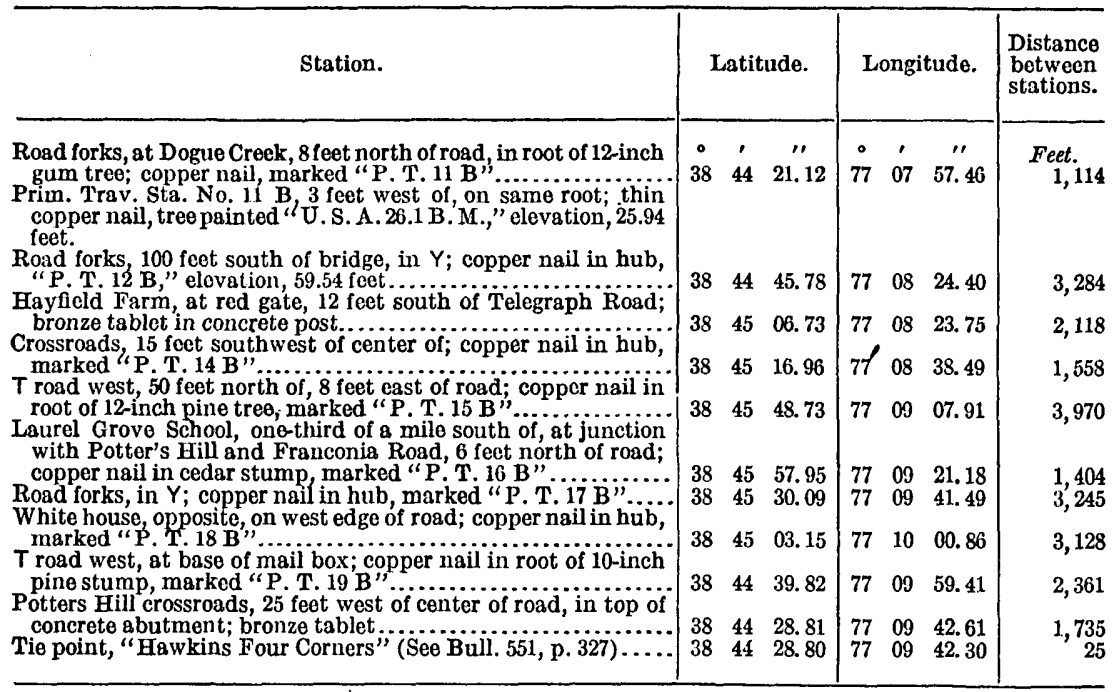

Magnetic declination, $4^{\circ} 20^{\prime} \mathrm{W}$.

From point near Laurel Grove School to point 2 miles southwest of Franconia.

"P. T. 16 B" (previously described)

Laurel Grove crossroads, 20 feet south of; copper nail in top of

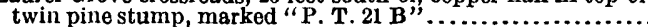

$T$ road east, at east edge of main road; copper nail in hub, marked "P. T. 22 B"

olivet chapol, $T$ road at, in root of oak tree used as sign board;

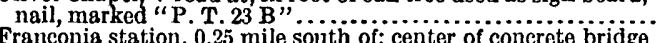
Franconia station, 0.25 mile south of; center of concrete bridge Sta. No 3 B" Ry. track, bronzo tablet stamped "Prim. Trav.

Road forks north, opposite white house, at north edge of road; $T$ road north, 20 feet east of at base of mail box; copper nail in $T$ road north, 20 feet east of, at base of mail box; copper nail in

Road forks northwest, 6 feet east of wood culvert; nail in hub

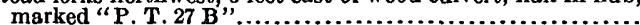
Dodson place, in front of, 25 feet east of road, in top of pine

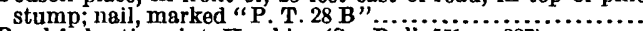

Road forks, tie point, Hawkins (See Buli. 551, p. 327 ).............

\begin{tabular}{lll|lll|r}
38 & 45 & 57.95 & 77 & 09 & 21.18 & $\ldots \ldots \ldots$ \\
38 & 46 & 13.30 & 77 & 09 & 12.77 & $.1,688$ \\
38 & 46 & 33.94 & 77 & 08 & 57.12 & 2,427 \\
38 & 46 & 55.80 & 77 & 08 & 46.83 & 2,358 \\
& & & & & & \\
38 & 46 & 46.38 & 77 & 09 & 24.95 & 3,180 \\
38 & 46 & 45.79 & 77 & 09 & 37.63 & 1,003 \\
38 & 46 & 41.18 & 77 & 10 & 05.68 & 2,270 \\
38 & 46 & 42.01 & 77 & 10 & 43.47 & 2,993 \\
38 & 46 & 18.41 & 77 & 10 & 52.52 & 2,494 \\
38 & 46 & 04.50 & 77 & 11 & 04.60 & 1,703 \\
\hline
\end{tabular}

Magnetic declination, $4^{\circ} 57^{\prime} \mathrm{W}$.

Three positions not tied in.

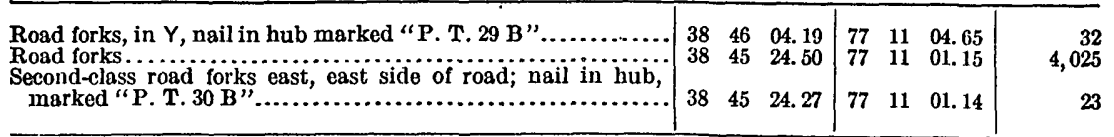

From crossroads at Pohick School southeast to Lorton. (By School of Topography, U. S. Army, in 1918. By D. S. Birkett in 1920.)

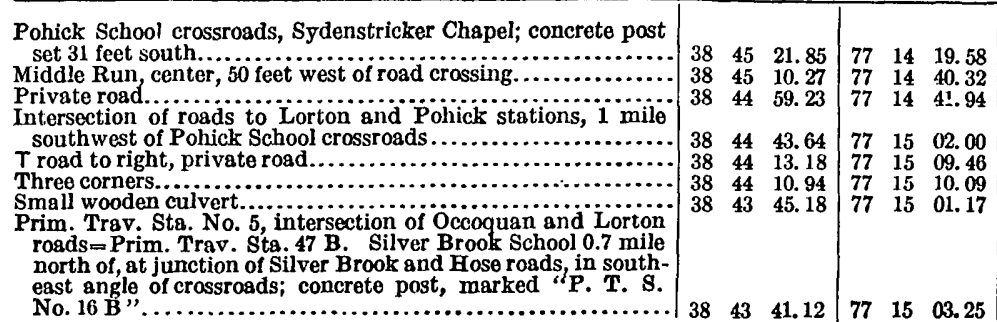


CAMP HUMPHREYS, INCLUDING PARTS OF FAIRFAX, INDIAN HEAD, OCCOQUAN, AND WASHINGTON QUADRANGLES-Continued.

From crossroads at Pohick School southeast to Lorton-Continued.

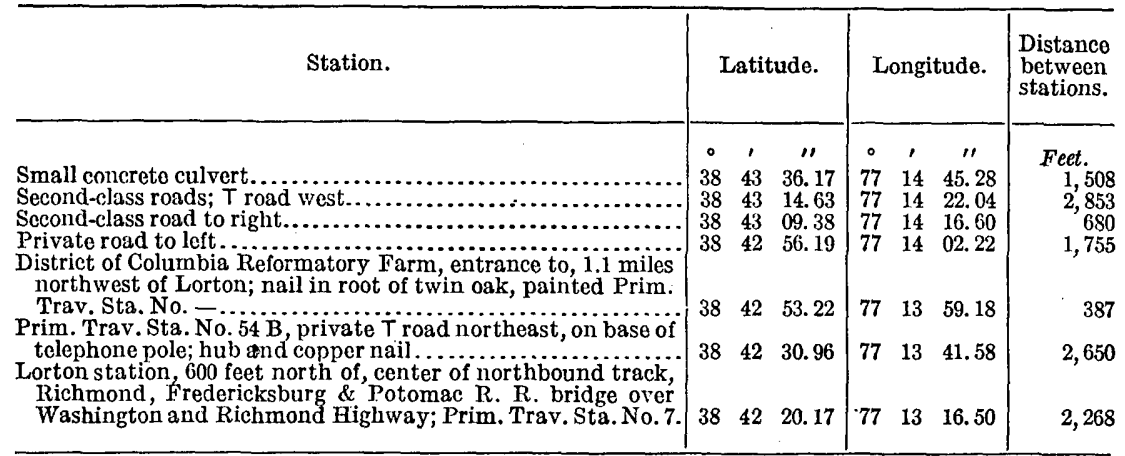

Magnetic declination, $5^{\circ} 32^{\prime} \mathrm{W}$.

From Lorton via Occoquan Workhouse and Silver Brook School to point on Rolling Road 2 miles north of Pohick School.

Lorton, Prim. Trav. Sta. No. 38 B, opposite Lorton Telephone Co.'s office, at base of telephone pole; hub and copper nail...

Prim. Trav. Sta. No. 39 B, bridge over Mill Creek, in floor near

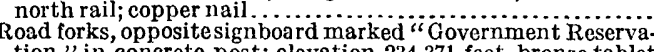
tion," in concrete post; elevation 234.371 feet, bronze tablet

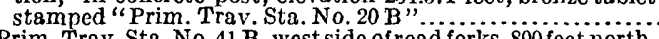

Prim. Trav. Sta. No. $41 \mathrm{~B}$, west side of road forks, 800 feet nor thwest of District of Columbia Reformatory stables; chiseled Prim. Trav. Sta. No. 42 B, T road, 1,000 feet northwest of District of Columbia farm watch tower, at base of signboard marked "To Washington," on top of cement curb; chiseled

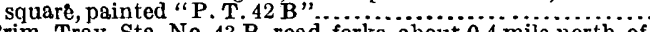

Prim. Trav. Sta. No. $43 \mathrm{~B}$, road forks, about 0.4 mile north of District of Columbia Reformatory buildings, at foot of 10 inch oak tree in Y, painted "P. T. 43 B" ............................ District of Columbia, old Reformatory boundary post at road forks........... No. $45 \mathrm{~B}, 25$ feet north of $\mathrm{Giles}$ Run, west edge of road, in top of 10 -inch oak stump; copper nail............. Prim. Trav. Sta. No. $46 \mathrm{~B}$, road forks, about 600 feet southwest of white house. $\mathrm{No} .47 \mathrm{~B}$, old point Prim. Trav. Sta. No. 5

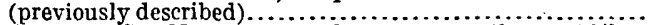

Prim. Trav. Sta. No. $48 \mathrm{~B}, \mathrm{~T}$ road, $, 1, \ldots 0$ feet southeast of Silver

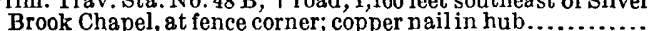
Prim. Trav. Sta. No. $49 \mathrm{~B}$, on root of 12 -inch oak tree, $0.3 \mathrm{mile}$ south of South Run, on north slope of hill, 30 feet east of fence.

Prim. Trav. Sta. No. $50 \mathrm{~B}$, at road intersection, south edge of third-class road. in root of twin oak tree; copper nail .........

Prim. Trav. Sta. No. 51 B, opposite gate at $T$ road north, south side of road. in top of 4 -inch oak stump; copper nail..........

Prim. Trav. Sta. No. 52 B, south edge of road, 100 feet east of Pohick Creek, south edge of field; copper nail in top of 3-inch

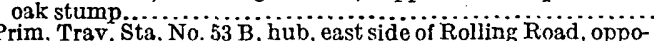

Prim. Trav. Sta. No. $53 \mathrm{~B}$, hub, east side of Rolling Road, opposite T road, on base of 12 -inch stump 7 feet high; copper nail.
old Prim. Trav. Sta. No. 2 , top of 8 -inch pine stump (previously described)

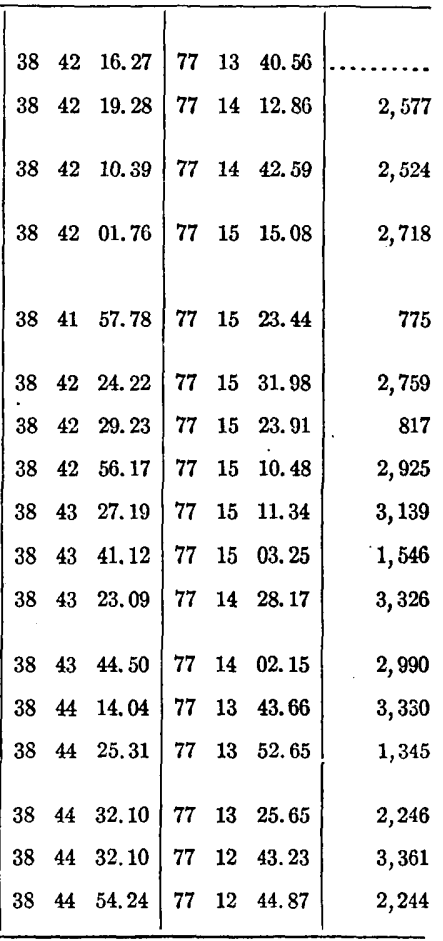

Magnetic declination, $5^{\circ} 30^{\prime} \mathrm{W}$.

Coutinuation of line from Gum Springs to junction of gravel and telegraph roads.

Prim. Trav. Sta. No. 8 B, opposite white house, 3 feet northwest

Prim. Trav. Sta. No. $9 \mathrm{~B}, 150$ feet south of brook, 5 feet east of

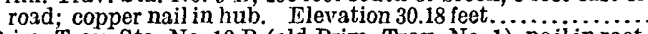

Prim. Trav. Sta. No. $10 \mathrm{~B}$ (old Prim. Trav. No. 1 ), nail in root

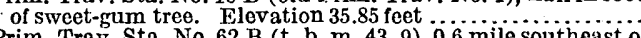

Prim. Trav. Sta. No. 62 B (t. b. m. 43.9 ), 0.6 mile southeast of forks of gravel and telegraph roads; copper nail in root of

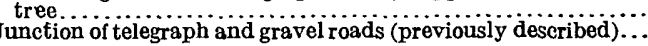

\begin{tabular}{|lll|lll|l}
38 & 44 & 36.48 & 77 & 07 & 29.73 & 1,772 \\
38 & 44 & 54.52 & 77 & 07 & 14.25 & 2,198 \\
38 & 45 & 06.72 & 77 & 07 & 03.83 & 1,484 \\
& & & & & & \\
38 & 45 & 41.10 & 77 & 07 & 01.50 & 3,479 \\
38 & 45 & 02.35 & 77 & 07 & 17.25 & 2,482
\end{tabular}


CAMP HUMPHREYS, INCLUDING PARTS OF FAIRFAX, INDIAN HEAD, OCCOQUAN, AND WASHINGTON QUADRANGLES-Continued.

Along highway from New Alexandria northwest to Fairfax court house. (By H. S. Senseney in 1920.)

\begin{tabular}{|c|c|c|c|c|c|c|c|}
\hline Station. & \multicolumn{3}{|c|}{ Latitude. } & \multicolumn{3}{|c|}{ Longitude. } & \multirow{2}{*}{$\begin{array}{c}\begin{array}{c}\text { Distance } \\
\text { between } \\
\text { stations. }\end{array} \\
\text { Feet. }\end{array}$} \\
\hline & & , & $\prime \prime$ & $\circ$ & , & $\prime \prime$ & \\
\hline Prim. Trav. Sta. No. $23 \mathrm{~S}$ (previously described) ................. & 38 & 47 & 28.56 & 77 & 03 & 38.32 & \\
\hline 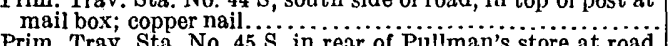 & 38 & 47 & 38.69 & 77 & 04 & 15.40 & 3,112 \\
\hline 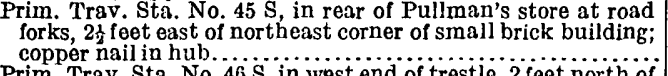 & 38 & 47 & 54.45 & 77 & 04 & 48.39 & 3,060 \\
\hline 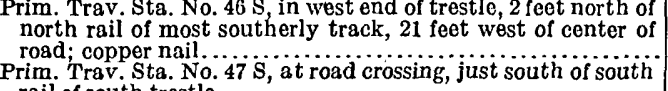 & 38 & 48 & 16.34 & 77 & 04 & 27.80 & 2,750 \\
\hline 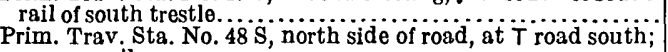 & 38 & 48 & 19.14 & 77 & & 16. 66 & 3,880 \\
\hline 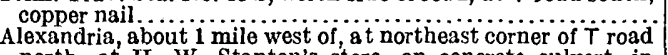 & 38 & 48 & 29.82 & 77. & 05 & 15.06 & 1,086 \\
\hline 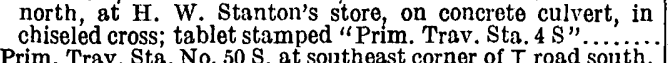 & 38 & 48 & 30.77 & 77 & 05 & 19.70 & 380 \\
\hline in top of stump; copper nail . & 38 & 48 & 36.77 & 77 & 05 & 52.60 & 2,675 \\
\hline 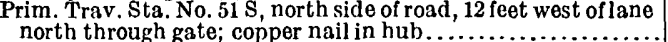 & & & & & & & \\
\hline Prim. Trav. Sta. No. $52 \mathrm{~s}$, north side of road, 12 feet west of & 8 & 48 & 77 & 77 & 0 & & 2,902 \\
\hline $\begin{array}{l}\text { lane through gate, in top of stump; copper nail } \\
\text { Prim. Trav. Sta. No. } 53 \mathrm{~S} \text {, on west side of lane north, } 5 \text { feet north }\end{array}$ & 38 & 48 & 42.59 & 77 & 07 & 01.94 & 2,687 \\
\hline $\begin{array}{l}\text { of road gate, in root of tree; copper nail......................... } \\
\text { Lincolnia, Prim. Trav. Sta. No. } 54 \mathrm{~S} \text {, at northeast corner of } \\
\text { crossroads, } 2.6 \text { feet south of tree; copper nail in hub (post No. } 5\end{array}$ & 38 & 48 & 46.38 & 77 & 07 & 30.95 & 2,329 \\
\hline $\begin{array}{l}\text { to be set here). } \\
\text { Prim. Trav. Sta. No. } 55 \mathrm{~S} \text {, at northeast corner of } \mathrm{T} \text { road north, }\end{array}$ & 38 & 48 & 56.26 & 77 & 08 & & \\
\hline $\begin{array}{l}\text { in root of tree. } \\
\text { Prim. Trav. Sta. No. } 56 \mathrm{~s} \text {, on south side of road, } 6 \text { feet north of }\end{array}$ & 38 & 49 & 06.24 & 77 & 08 & 36.50 & 2,105 \\
\hline 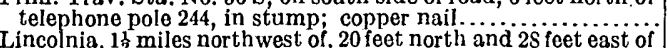 & 38 & 49 & 12.33 & 77 & 09 & 11.45 & 2,834 \\
\hline $\begin{array}{l}\text { crossroads, Prim. Trav. Sta. No. 57 S; copper nail in hub } \\
\text { (post No. } 6 \text { to be set here)............................... }\end{array}$ & 38 & 49 & 21.58 & 77 & 09 & 48.72 & 3,097 \\
\hline $\begin{array}{l}\text { feet south of fence, in top of stump; copper nail..... } \\
\text { Prim. Trav. Sta. No. } 59 \mathrm{~S} \text {, north side of road, } 40 \text { feet east of } \mathrm{G} . \mathrm{H} \text {. }\end{array}$ & 38 & 49 & 30.71 & 77 & 10 & 27.83 & 3,232 \\
\hline 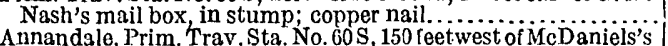 & 38 & 49 & 42.35 & 77 & 11 & 06.40 & 3,273 \\
\hline $\begin{array}{l}\text { store, on south side of road, } 30 \text { feet west of road forks; copper } \\
\text { nail in hub (post No. } 7 \text { to be set here)..................... } \\
\text { Prim. Trav. Sta. No. } 61 \text { S, } 4 \text { feet west of telephone pole } 369 \text { in }\end{array}$ & 38 & 49 & 49.94 & 77 & 11 & 49.88 & 3,527 \\
\hline $\begin{array}{l}\text { top of stump; copper nail. . } \\
\text { Prim. Trav. Sta. No. } 62 \mathrm{~S} \text {, south side of road, } 20 \text { feet east of } \mathrm{T}\end{array}$ & 38 & 49 & 56.25 & 77 & 12 & 26.84 & 2,995 \\
\hline $\begin{array}{l}\text { road north, } 3 \text { feet north of fence post; copper nail in hub..... } \\
\text { Prim. Trav. Sta. No. } 63 \mathrm{~S} \text {, cross cut in south end of east abut- }\end{array}$ & 38 & 50 & 04.08 & 77 & 13 & 05.54 & 3,163 \\
\hline 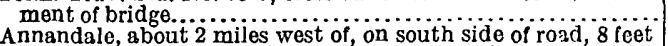 & 38 & 50 & 08.63 & 77 & 13 & 39.46 & 2,725 \\
\hline 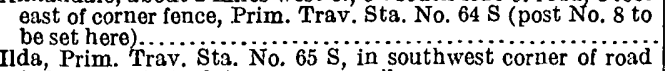 & 38 & 50 & 12.48 & 77 & 14 & 20.77 & 3,293 \\
\hline forks, in root of oak tree; copper nail ........................... & 38 & 50 & 18.17 & 77 & 15 & 06.38 & 3,656 \\
\hline 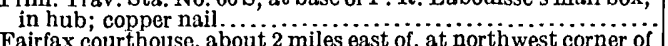 & 38 & 50 & 23.16 & 77 & 15 & 42.35 & 2,891 \\
\hline 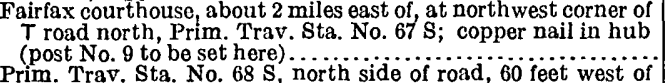 & 38 & 50 & 29.05 & 77 & 16 & 25.30 & 3,450 \\
\hline $\begin{array}{l}\text { house, in stump; copper nail. } \\
\text { Prim. Trav. Sta. No } 69 \mathrm{~S} \text {, north side of road, } 60 \text { feet west of } \ddot{\mathrm{T}}\end{array}$ & 38 & 50 & 34.33 & 77 & 17 & 06.09 & 3,273 \\
\hline 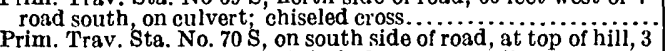 & 38 & 50 & 35.88 & 77 & 17 & 18.82 & 1,019 \\
\hline feet west of white gatepost, in hub; copper nail.. & 38 & 50 & 39.92 & 77 & 17 & 52.37 & 2,687 \\
\hline
\end{tabular}

From Fairfax south along highways to Occoquan.

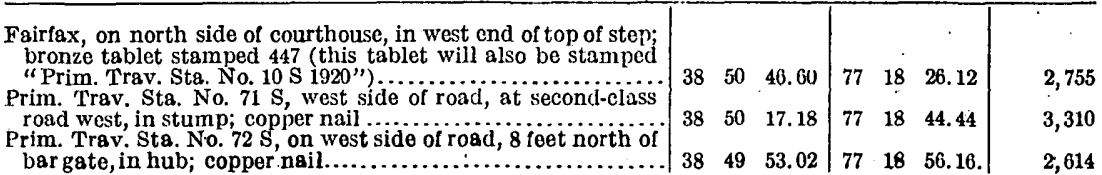


CAMP HUMPHREYS, INCLUDING PARTS OF FAIRFAX, INDIAN HEAD, OCCOQUAN, AND WASHINGTON QUADRANGLES-Continued.

From Fairfax south along highways to Occoquan-Continued.

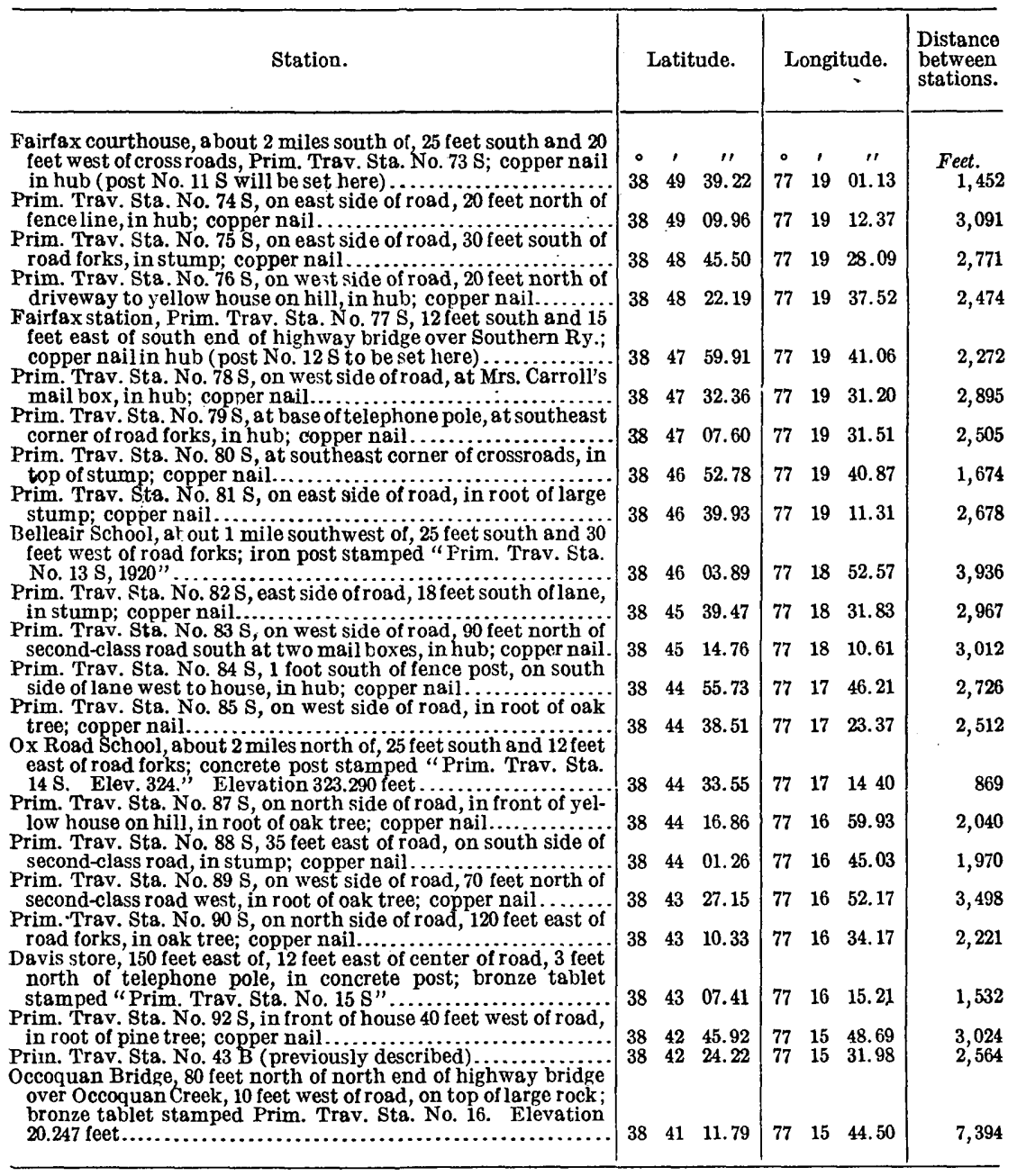

From Lorton south along Southern Ry. to Colchester and west and north to Washington and Richmond highway. (By D. S. Birkett in 1920.)

Lorton siation, about 600 feet north of (previously described)... Lorton station, center line of track ..........................

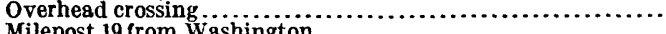

Colchester, Prim. Trav, Sta. No. 1 on concrete abutment of un.

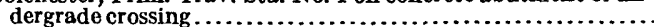
Occoquan railroad bridge, on curtain wail abutment, east end of bridge; painted square .

Occoquan bridge, center line.

Colchester station, 0.4 mile west of; Prim. Trav. Sta. No. $\ddot{35}$ B 5 feet west of second-class road up Occoquan Creek, at edge of field, in top of 12-inch oak stump; copper nail. Elevation $\mathbf{8 5 . 4 6}$ leet.

Prim. Trav, Sta. No 36 B 800 feet west of rosd forks, on concrete cuivert over smail creek, south ond; chiseled square. Eleva-

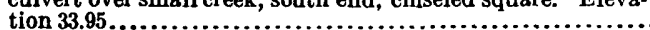

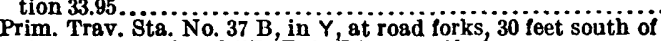

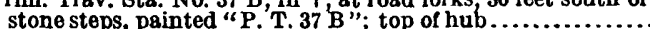

\begin{tabular}{rrr|rrr|r}
38 & 42 & 20.17 & 77 & 13 & 16.50 & $\ldots \ldots, \ldots 20$ \\
38 & 42 & 10.37 & 77 & 13 & 19.68 & 1,020 \\
38 & 41 & 48.53 & 77 & 13 & 26.77 & 2,280 \\
38 & 41 & 03.53 & 77 & 13 & 39.79 & 4,667 \\
38 & 40 & 16.29 & 77 & 14 & 05.67 & 5,200 \\
38 & 40 & 04.90 & 77 & 14 & 22.53 & 1,766 \\
38 & 40 & 07.90 & 77 & 14 & 18.09 & 464 \\
& & & & & & \\
38 & 40 & 23.32 & 77 & 13 & 23.36 & 1,574 \\
38 & 40 & 35.66 & 77 & 14 & 41.63 & 1,914 \\
38 & 40 & 43.53 & 77 & 14 & 52.30 & 1,162
\end{tabular}


CAMP HUMPHREYS, INCLUDING PARTS OF FAIRFAX, INDIAN HEAD, OCCOQUAN, AND WASHINGTON QUADRANGLES-Continued.

From Lorton south along Southern Ry. to Colchester and west and north to Washington and Richmond Highway-Continued.

\begin{tabular}{|c|c|c|c|}
\hline Station. & Latitude. & Longitude. & $\begin{array}{l}\text { Distance } \\
\text { between } \\
\text { stations. }\end{array}$ \\
\hline 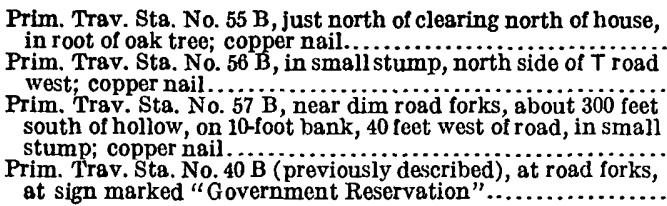 & $\begin{array}{ccc}\circ & \prime & \prime \prime \\
38 & 41 & 13.15 \\
38 & 41 & 25.52 \\
38 & 41 & 43.58 \\
38 & 42 & 10.39\end{array}$ & $\begin{array}{ccc}0 & 1 & \prime \prime \\
77 & 14 & 32.56 \\
77 & 14 & 15.32 \\
77 & 14 & 14.22 \\
77 & 14 & 42.59\end{array}$ & $\begin{array}{l}\text { Feet. } \\
\quad 3,383 \\
1,852\end{array}$ \\
\hline
\end{tabular}

Magnetic declination, $4^{\circ} 23^{\prime}$ W., 1920.

From forks of telegraph and gravel roads southwest to a point two miles southwest of Aloxandria. (By D. S. Birkett in 1920.)

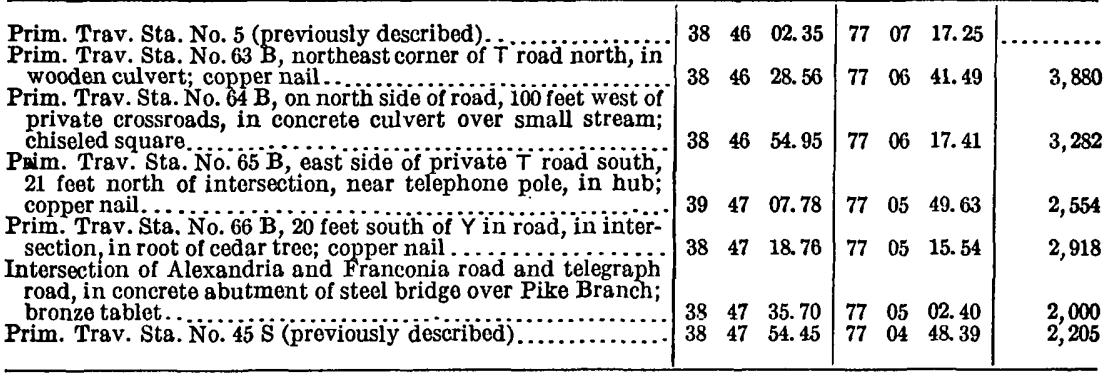

South from Lowis Chapel to Occoquan Bay. (By D. S. Birkett in 1920.)

Lewis Chapel crossroads (previously described).

Prim. Trav. Sta. No. 68 B, 14 feet east of T. B. M. $145.8,40$ feet north of dim road forks, 4,000 feet south of Lewis Chapel, on west side of road, in root of twin oak tree; copper nail.

Prim. Trav. Sta. No. $69 \mathrm{~B}$ temporary bench mark 138.3 onefourth of a mile south of Lewis Chapel, at road forks, 240 feet north of small house, on east side of road, in small stump; copper nail.

Prim. Trav. Sta. No. 70 B, at private-road crossing, 50 feet south of intersection, in very small stump; copper nail

Prim. Trav. Sta. No. 71 B 900 feet due southeast of $\mathrm{J}$. laip's house (Belmont Farm), 30 feet southwest of south corner of orchard fence, in concrete post, bronze tablet...........

\begin{tabular}{|lll|lll|r}
38 & 41 & 13.94 & 77 & 12 & 31.75 & $\ldots \ldots \ldots$ \\
38 & 40 & 34.44 & 77 & 12 & 30.26 & 3,998 \\
38 & 40 & 10.75 & 77 & 12 & 33.11 & 2,409 \\
38 & 39 & 53.95 & 77 & 12 & 29.98 & 1,718 \\
38 & 39 & 36.58 & 77 & 12 & 01.56 & 2,862
\end{tabular}

Magnetic declination, $5^{\circ} 57^{\prime} \mathrm{W}$.

Sonthwest from Lewis Chapel to a point on the Richmond, Frederlcksburg \& Potomac $\mathbf{R}$. $\mathbf{R}$. 1 mile north of Colchester.

Lewis Chapel crossroads (previously described)

Lewis Chapel, 0.5 mile southwest of, Prim. Trav. Sta. No. $7 \ddot{2} \ddot{\mathbf{B}}$ 45 feet northeast of intersection of private gravel roads, on west side of road, 100 feet west of smallstream; copper nail in stump.. Lewis Chapel, 0.75 mile southwest of, Prim. Trav. Sta.No. 73 B, opposite very old frame house (unoccupied) at top of steep hill, 8 feet north of gum tree, on south side of road, in hub;

copper nail. . . N No. 33 B in plank east side of second-class road crossing, about 1 mile north of Colchester on Richmond, Fredericksburg \& Potomac R. R.; copper nail.

Prim. Trav. Sta. No. 1, at Colchester station (previousiy de

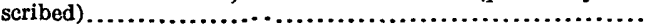

\begin{tabular}{|lll|lll|r}
38 & 41 & 13.94 & 77 & 12 & 31.75 & $\ldots \ldots \ldots$ \\
38 & 40 & 59.78 & 77 & 13 & 03.26 & 2,878 \\
38 & 40 & 47.60 & 77 & 13 & 15.97 & 1,591 \\
38 & 40 & 51.71 & 77 & 13 & 42.08 & 2,108 \\
38 & 40 & 16.29 & 77 & 14 & 05.67 & 4,042 \\
\hline
\end{tabular}

Magnetic declination, $5^{\circ} 56^{\prime} \mathrm{W}$ 
CAMP HUMPHREYS, INCLUDING PARTS OF FAIRFAX, INDIAN HEAD, OCCOQUAN, AND WASHINGTON QUADRANGIES-Continued.

Along highways from Barker's crossroads on Rolling Road and south to Sydenstricker Chapel (Pohick School). (By D. S. Birkett in 1920.)

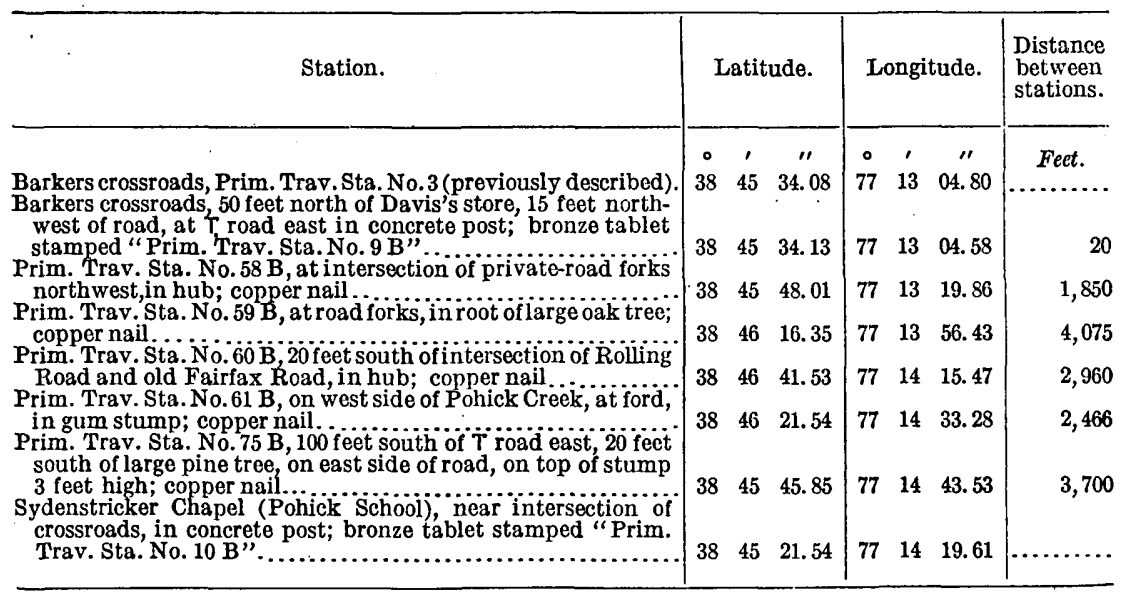

Magnetic declination, $5^{\circ} 42^{\prime} \mathrm{W}$.

West along Southern Ry. from Seminary station to Fairfax station. (By H. S. Senseney in 1920.)

Prim. Trav. Sta. No. $47 \mathrm{~S}$ (previously described)

Cameron Run 850 feet east of, on top of southwest corner of south abutment of Southern Ry. bridge, about 4 feet above track aluminum tablet stamped " 54 , Prim. Trav. Sta. No. $17 \mathrm{~S}$, 1920 ".

Lincolnshire station, 12 feet south and 25 feet west of road crossing, in hub P. T. $98 \mathrm{~S}$; copper nail. Edsall, 35 feet west of station, 23 feet south of south rail of south track, in concrete post; bronze tablet stamped "Prim. Trav. Sta. No. 19"

Springfield, 510 feet east of station, in west abutment of railroad bridge over Back Lick Run, in top of stone at south end; aluminum tablet stamped " 215 , Prim. Trav. Sta. No. $20 \mathrm{~S}$, 1920 "....................................................... Prim. Trav. Sta. No. 101 S., at southeast corner of second-class road crossing, on culvert; chiseled square.

Prim. Trav. Sta. No. $102 \mathrm{~S}$, in north end of east abutment of bridge over Accotink Creek; chiseled square................

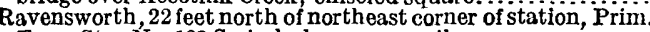

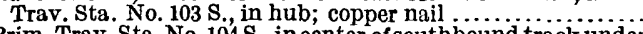
Prim. Trav. Sta. No. $104 \mathrm{~S}$, in center of southbound track under

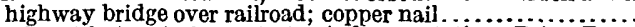

Burke, 30 feet east of operator's window at station, Prim. Trav. Sta. No. 105 S., in north ond of platform; copper nail.......... Burke, 0.25 mile west of, Prim. Trav. Sta. No, $106 \mathrm{~S}$., 15 feet south of track, 50 feet west of road crossing, 5 feet below line of track in top of southeast corner of concrete culvert; aluminum tablet stamped " 262 Prim. Trav. Sta. No. 22S, 1920 '........ Prim. Trav. Sta. No. $107 \mathrm{~S}$., near center of side track, on east edge of second-class road crossing, 230 feet east of trestle over

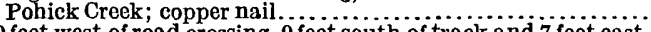

60 feet west of road crossing, 9 feet south of track and 7 feet east of telephonepole; in top of concrete post tablet stamped " $24 \mathrm{~S}$ " Sideburn, 25 feet east of station, Prim. Trav. Sta. No. 109 S., about 6 inches south of north rail of southbound track, in tie;

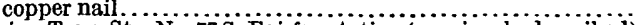

Prim. Trav. Sta. No. 77 S, Fairfax station (previousiy described).

\begin{tabular}{|c|c|c|c|c|c|c|}
\hline 38 & 48 & 19.14 & 77 & 05 & 16.66 & $\ldots$ \\
\hline 38 & 48 & 23.06 & 77 & 06 & 24.52 & 5,386 \\
\hline 38 & 48 & 05.51 & 77 & 08 & 02.90 & 7,986 \\
\hline 38 & 47 & 42.39 & 77 & 09 & 28.64 & 7,179 \\
\hline 38 & 47 & 48.11 & 77 & 11 & 00.70 & 7,309 \\
\hline 38 & 47 & 44.57 & 77 & 11 & 51.74 & 4,055 \\
\hline 38 & 47 & 34.28 & 77 & 13 & 03.09 & 5,743 \\
\hline 38 & 47 & 46.09 & 77 & 14 & 14.06 & 5,744 \\
\hline 38 & 47 & 45.32 & 77 & 14 & 55.31 & 3,265 \\
\hline 38 & 47 & 42.91 & 77 & 16 & 12.03 & 6,079 \\
\hline 38 & 47 & 51.33 & 77 & 16 & $\begin{array}{c}31.39 \\
=\end{array}$ & 1,75 \\
\hline 38 & 47 & 53.97 & 77 & 17 & 02.39 & 2,466 \\
\hline 38 & 47 & 51.06 & 77 & 18 & 06.68 & 5,098 \\
\hline $\begin{array}{l}38 \\
38\end{array}$ & $\begin{array}{l}48 \\
47\end{array}$ & $\begin{array}{r}00.33 \\
59.91\end{array}$ & $\begin{array}{l}77 \\
77\end{array}$ & $\begin{array}{l}18 \\
19\end{array}$ & $\begin{array}{l}33.38 \\
41.06\end{array}$ & $\begin{array}{l}2,312 \\
5,356\end{array}$ \\
\hline
\end{tabular}

From Springfleld station south on highway about 11 miles.

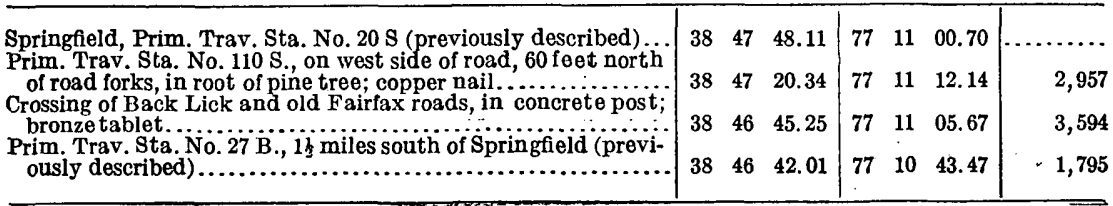


CAMP HUMPHREYS, INCLUDING PARTS OF FAIRFAX, INDIAN HEAD, OCCOQUAN, AND WASHINGTON QUADRANGLES-Continued.

From a point 1.25 miles south of Lincolnia along highway to Lincolnia.

\begin{tabular}{|c|c|c|c|c|c|c|}
\hline $\begin{array}{c}\text { Station. } \\
\text { Prim. Trav. Sta. No. } 23 \text { B (previously described) ............. } \\
\text { Prim. Trav. Sta. No. } 112 \text { S, on south side of road at Troad north, }\end{array}$ & \multicolumn{2}{|c|}{ Latitude. } & \multicolumn{3}{|c|}{ Longitude. } & $\begin{array}{l}\text { Distance } \\
\text { between } \\
\text { stations. } \\
\text { Feet. }\end{array}$ \\
\hline 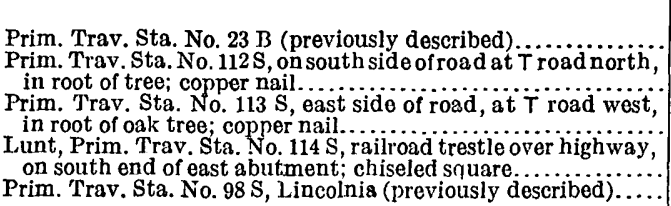 & $\begin{array}{cc}0 & 1 \\
38 & 46 \\
38 & 47 \\
38 & 47 \\
38 & 47 \\
38 & 48\end{array}$ & $\begin{array}{l}55.80 \\
02.51 \\
37.71 \\
54.30 \\
05.51\end{array}$ & $\begin{array}{l}0 \\
77 \\
77 \\
77 \\
77 \\
77\end{array}$ & $\begin{array}{l}1 \\
08 \\
08 \\
08 \\
\\
08 \\
08\end{array}$ & $\begin{array}{c}\prime \prime \\
46.63 \\
11.05 \\
13.96 \\
\\
04.48 \\
02.90\end{array}$ & $\begin{array}{r}\text { Feet. } \\
\ldots \ldots \ldots \\
2,805 \\
3,570 \\
1,839 \\
1,144\end{array}$ \\
\hline
\end{tabular}

From a point 1 mile north of the $0 x$ Road School east to crossrosds, 0.5 mile northwest of Silver Brook School. (By D. S. Birkett in 1920.)

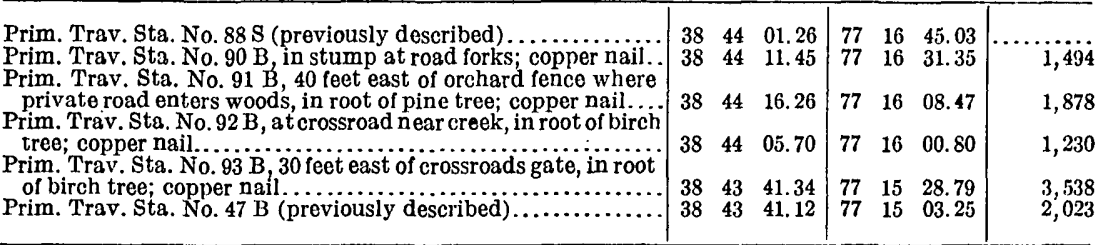

Magnetic declination, $5^{\circ} 46^{\prime} \mathrm{W}$.

From Olivet Chapel southeast via Triplett place to forks of Gravel and Telegraph roads.

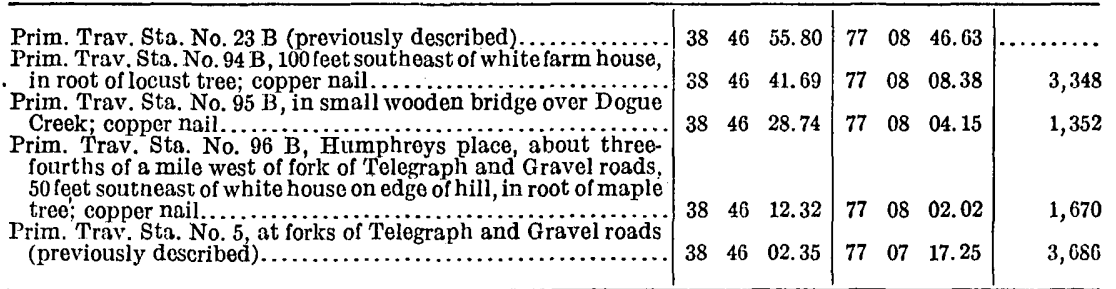

Magnetic declination, $4^{\circ} 22^{\prime} \mathrm{W}$.

From crossroads 1.5 miles south of Burke southwest to Ox Road School. (By D. S. Birkett in 1920.)

\begin{tabular}{|c|c|c|c|c|c|c|c|}
\hline Prim. Trav. Sta. No. 88 B (previously described).... & & 46 & 37.09 & 77 & 16 & 33.48 & \\
\hline 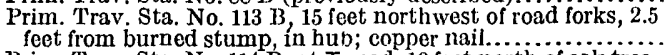 & 38 & 46 & 23.75 & & 17 & 08.16 & 3,062 \\
\hline $\begin{array}{l}\text { Prim. Trav. Sta. No. } 114 \text { B, at; T road, } 10 \text { feet north of oak tree, } \\
15 \text { feet west of center line of road, in hub; copper nail.......... }\end{array}$ & 38 & 46 & 06.90 & 77 & 17 & 19.00 & \\
\hline $\begin{array}{l}\text { Prim. Trav. Sta. No. } 115 \mathrm{~B} \text {, at road forks, in oak root; copper nail. } \\
\text { Prim. Trav. Sta. No. } 116 \mathrm{~B} \text {, at center of road forks, } 15 \text { feet south- }\end{array}$ & 38 & 45 & 47.85 & 77 & 17 & 22.68 & \\
\hline $\begin{array}{l}\text { west to marked tree, in hub; copper nail. } \\
\text { Prim. Trav. Sta. No. } 117 \mathrm{~B}, 60 \text { feet north of creek at ford, } 5 \text { feet }\end{array}$ & 38 & 45 & 27.89 & 77 & 17 & 41.34 & \\
\hline west of center line of road; copper nail in ash root............ & 38 & 45 & 07.07 & 77 & 17 & 33.40 & 2 \\
\hline 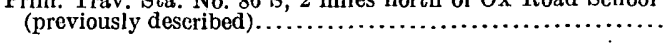 & & & 33.55 & 77 & 17 & 14.40 & 3,710 \\
\hline
\end{tabular}

Magnetic declination, $5^{\circ} 56^{\prime} \mathrm{W}$.

From Lee Chapel to point 1 mile northwest of Pohick School. (By D. S. Birkett in 1920.)

U. S. Geological Survey permanent benclı mark; Lee Chapel (Prim. Trav. Sta. No. 2, 1911) (previously described)......... Prim. Trav. Sta. No. 118 B, at T road, in pine root; copper nail Prim. Trav. Sta. No. 119 B, 300 feet west to creek, 60 feet west of old mill barracks, in stump; copper nail................. of center of road in oak root; copper nail.................

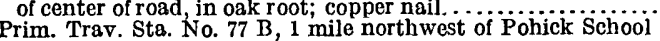

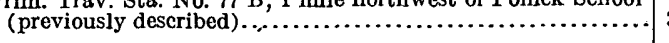

\begin{tabular}{|lll|lll|r}
38 & 45 & 19.75 & 77 & 16 & 37.25 & $\ldots \ldots \ldots$ \\
38 & 45 & 06.81 & 77 & 16 & 05.94 & 2,804 \\
38 & 45 & 22.11 & 77 & 15 & 48.92 & 2,062 \\
38 & 45 & 33.00 & 77 & 15 & 17.68 & 2,715 \\
38 & 45 & 58.39 & 77 & 15 & 09.94 & 2,646
\end{tabular}

Magnetic declination, $5^{\circ} 48^{\prime} \mathrm{W}$. 
CAMP HUMPHREYS, INCLUDING PARTS OF FAIRFAX, INDIAN HEAD, OCCOQUAN, AND WASHINGTON QUADRANGIES-Continued.

From point near Gunston Hall to Sandy Point. (U. S. C. \& G. S. station.)

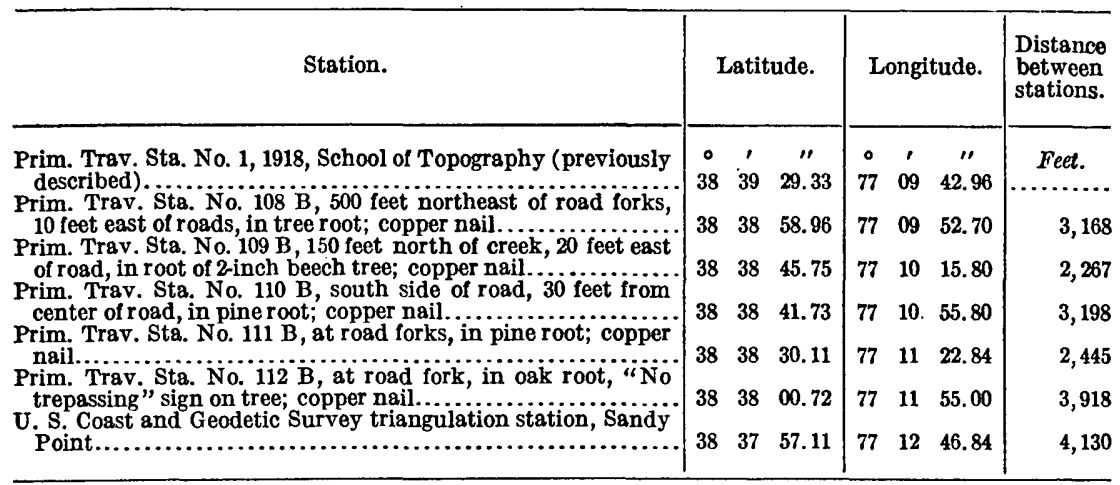

Magnetic declination, $5^{\circ} 37^{\prime} \mathrm{W}$.

From a point about 2 miles southwest of Alexandria west along old Fairfax Road.

Prim. Trav. Sta. No. 67 B (previously described)

Prim. Trav. Sta. No. 74 B, forks of Alexandria and Franconia road, 0.7 mile west of private $T$ road north, east end of gate, 25 feet east of intersection, in stump near telephone pole;

copper nail.................... of old Fairfax Road, in root of large cherry tree: copper nail..

Prim. Trav. Sta. No. 83 B, on south side of road, about 170 feet east of large frame house south of road, on top of concrete culvert; chiseled square...................................

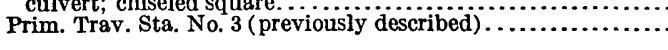

Magnetic declination, $5^{\circ} 32^{\prime} \mathrm{W}$.

From a point 1.6 miles south of Lee Chapel over highways to Burke.

Prim. Trav. Sta. No. $86 \mathrm{~B}$ (previously described).

Prim. Trav. Sta. No. 84, in south corner of bridge over South Run; copper nail. Elevation 323.78 feet......................... U. S. Geological Survey position at Lee Chapel, Prim. Trav. Sta. Prim. Trav. Sta. No. $85 \mathrm{~B}, 10$ feet east of creek, 8 feet south of road, in stump; copper nail............................... Prim. Trav. Sta. No. $86 \mathrm{~B}, 50$ feet west of road fork, in root of

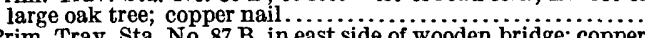

Prim. Trav. Sta. No. 87 B, in east side of wooden bridge; copper

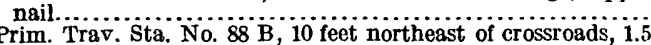

Prim. Trav. Sta. No. $88 \mathrm{~B}, 10$ feet northeast of crossroads, 1.5
miles south of Burke, about 100 feet west of large dead tree,

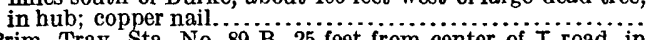

Prim. Trav. Sta. No. $89 \mathrm{~B}, 25$ feet from center of $\mathrm{T}$ road, in

northeast corner near fence post, in hub; copper nail.........
rim. Trav. Sta. No. 105 B, 600 feet west of Burke, northeast

Prim. Trav. Sta. No. 105 B, 600 feet west of Burke, northeast
abutment of iron bridge, in sandstone rock; chiseled square..

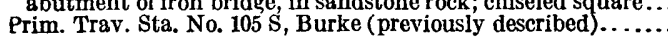

\begin{tabular}{lll|lll|r}
38 & 47 & 35.65 & 77 & 05 & 02.48 & $\ldots \ldots \ldots \ldots$ \\
38 & 47 & 29.81 & 77 & 05 & 50.42 & 3,839 \\
38 & 47 & 29.58 & 77 & 06 & 08.79 & 1,452 \\
& & & & & & \\
38 & 47 & 19.81 & 77 & 06 & 46.70 & 3,160 \\
38 & 47 & 10.63 & 77 & 07 & 20.30 & 2,816 \\
\hline
\end{tabular}

3,839

1,452

160

.

\begin{tabular}{|lll|lll|r}
38 & 44 & 33.55 & 77 & 17 & 14.40 & $\ldots \ldots \ldots$ \\
38 & 44 & 50.80 & 77 & 16 & 55.28 & 2,313 \\
38 & 45 & 19.75 & 77 & 16 & 37.25 & 3,259 \\
38 & 45 & 31.32 & 77 & 16 & 24.90 & 1,527 \\
38 & 45 & 52.57 & 77 & 16 & 22.74 & 2,158 \\
38 & 46 & 12.43 & 77 & 16 & 19.82 & 2,022 \\
& & & & & \\
38 & 46 & 37.09 & 77 & 16 & 33.48 & 2,720 \\
38 & 47 & 01.91 & 77 & 16 & 46.57 & 2,718 \\
38 & 47 & 33.25 & 77 & 16 & 24.28 & 3,630 \\
38 & 47 & 42.91 & 77 & 16 & 12.05 & 1,376 \\
\hline
\end{tabular}

Magnetic declination, $6^{\circ} 01^{\prime} \mathrm{W}$.

\section{From point near Lewis Chapel to Cone Creek.}

Lewis Chapel crossroad, 50 feet southwest of (previously de-

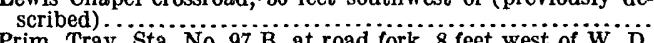
Prim. Trav. Sta. No. $97 \mathrm{~B}$, at road fork, 8 feet west of $\dddot{w} .{ }_{\mathrm{D}}$.
Dean's mail box, in top of concrete post; tablet stamped Dean's mail box, in top of concrete post; tablet stamped

Prim. Trav. sta. No. 98 B, 100 feet east of house, at road turn,

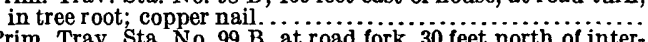

Prim. Trav. Sta. No. $99 \mathrm{~B}$, at road fork, 30 feet north of in ter-

section; copper nail in stump..................................

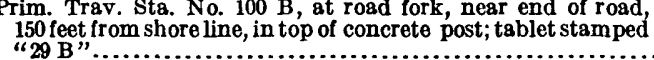

\begin{tabular}{|rrr|rrr|r}
38 & 41 & 13.94 & 77 & 12 & 31.73 & $\ldots \ldots \ldots .$. \\
38 & 40 & 07.50 & 77 & 11 & 14.02 & 9,119 \\
38 & 39 & 36.70 & 77 & 11 & 10.28 & 3,131 \\
38 & 39 & 20.94 & 77 & 11 & 12.59 & 1,604 \\
38 & 39 & 08.65 & 77 & 11 & 37.89 & 2,362 \\
\hline
\end{tabular}


CAMP HUMPHREYS, INCLUDING PARTS OF FAIRFAX, INDIAN HEAD, OCCOQUAN, ÁND WASHINGTON QUADRANGLES-Continued.

From Edsall to Franconia.

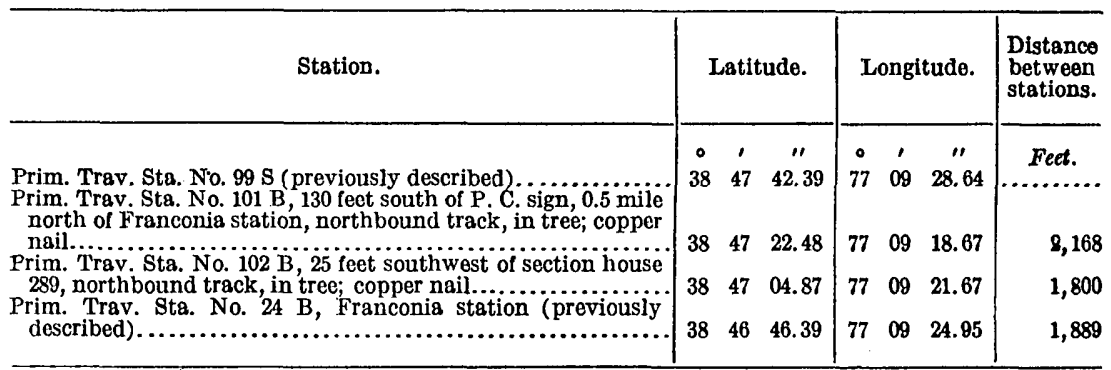

Magnetic declination, $5^{\circ} 00^{\prime} \mathrm{W}$.

From point 2 miles west of Franconla northwest to Southern Ry.

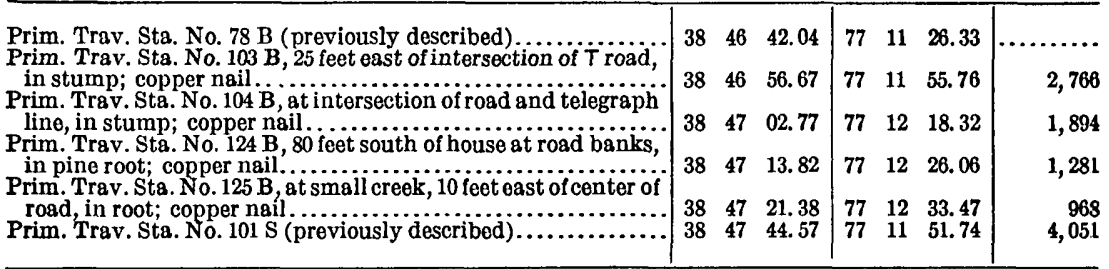

Magnetic declination, $5^{\circ} 31^{\prime} \mathrm{W}$

From.point 1 mile northwest of Pohick School to point 1.5 milles south of Burke.

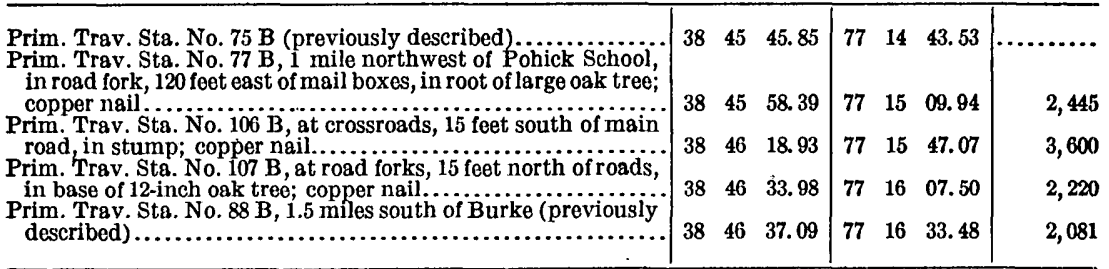

Magnetic declination, $5^{\circ} 44^{\prime} \mathrm{W}$.

From point 1.3 miles west of Franconla west along old Falrfax Road.

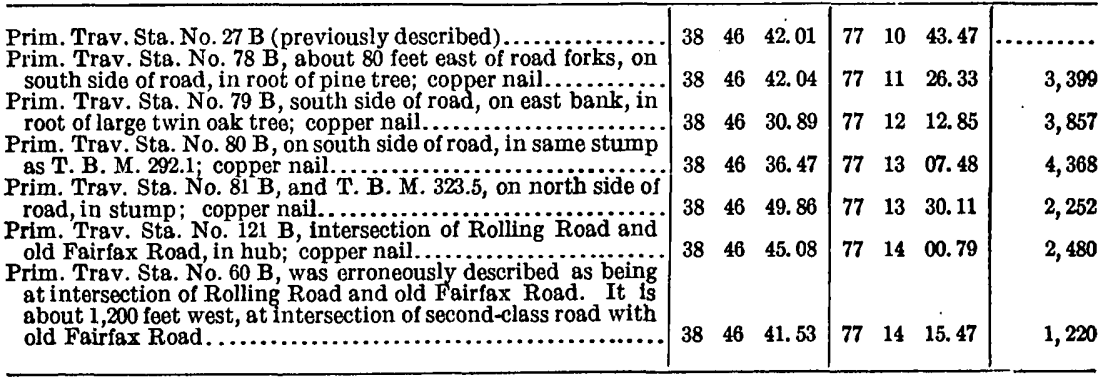

Magnetic declination, $5^{\circ} 35^{\prime} \mathrm{W}$. $121166^{\circ}-23-$ Bull. $709-29$ 
CAMP HUMPHREYS, INCLUDING PARTS OF FAIRFAX, INDIAN HEAD, OCCOQUAN, AND WASHINGTON QUADRANGLES-Continued.

From intersection of old Fatrfax Road and Rolling Road north to Ravensworth.

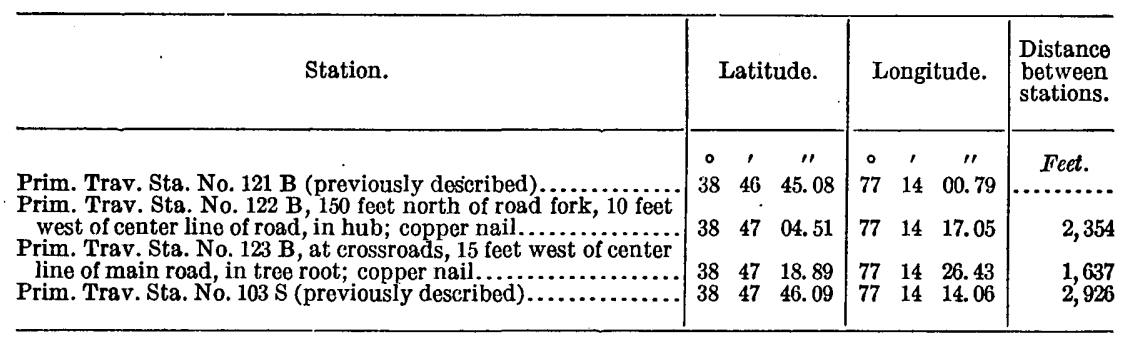

Magnetic declination, $5^{\circ} 48^{\prime} \mathrm{W}$.

From a point on Rolling Road 1.5 miles north of Pohick station east to Accotink station. (By D. S. Birkett in 1920.)

Prim. Trav. Sta. No. 53 B (previously described)

Prim. Trav. Sta. No. 126 B, northwest corner of field, 4 feet west

of center of road, copper nail in stump.....................

Prim. Trav. Sta. No. 127 B, 125 feet north of Accotink Creek, at

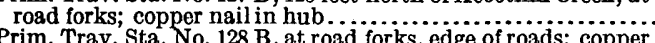

Prim. Trav. Sta. No. $128 \mathrm{~B}$, at road forks, edge of roads; copper

point on northbound track of Richmond, Fredericksburg \&

Potomac R. R., opposite tangent on Camp A. A. Humphreys

R. R., School of Topography point, 1918 (previously described)

\begin{tabular}{rrr|rrr|r}
38 & 44 & 32.10 & 77 & 12 & 43.23 & $\ldots \ldots \ldots$ \\
38 & 44 & 12.52 & 77 & 12 & 28.88 & 2,285 \\
38 & 43 & 59.75 & 77 & 12 & 05.55 & 2,259 \\
38 & 43 & 51.48 & 77 & 12 & 00.12 & 944 \\
38 & 44 & 06.12 & 77 & 11 & 12.90 & 4,027
\end{tabular}

From a point on Alexandria and Franconia road north via Bush Fill to crossing of Southern $\mathbf{R}$. and Richmond, Fredericksburg \& Potomac $\mathbf{R}$. $\mathbf{R}$.

Prim. Trav. Sta. No. $82 \mathrm{~B}$ (previously described) ............

Prim. Trav. Sta. No. 129 B, north edge of hill, about 600 feet northwest of brown frame house (Hadden place), west edge of private road, in root of oak tree; copper nail................. 40 feet north of creek north edge of road; copper nail in hub. Cameron Run, 850 feet east of; aluminum tablet No. 54 (pre-

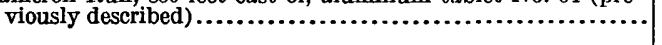

\begin{tabular}{|lll|lll|r}
38 & 47 & 29.58 & 77 & 06 & 08.79 & $\ldots \ldots \ldots$ \\
38 & 47 & 52.23 & 77 & 06 & 07.74 & 2,293 \\
38 & 48 & 05.80 & 77 & 06 & 15.87 & 1,520 \\
38 & 48 & 23.06 & 77 & 06 & 24.52 & 1,877 \\
\hline
\end{tabular}

From Laurel Grove School east about 1 mile.

NoTE.-This line was not completed; thero was no azimuth nor distance check, and the positions are preliminary.

Prim. Trav. Sta. No. 21 B (previously described)

Prim. Trav. Sta. No. $131 \mathrm{~B}$, about 600 feet southeast of white

frame house, in fork of roads; copper nail in hub.............

Station 1914 , hub on east side of road . . . . . . . . . . . . . . .

\begin{tabular}{|rrr|rrr|r}
38 & 46 & 13.30 & 77 & 09 & 12.77 & $\ldots \ldots \ldots \ldots$ \\
38 & 46 & 06.29 & 77 & 08 & 42.63 & 2,490 \\
38 & 45 & 59.83 & 77 & 08 & 31.94 & 1,072
\end{tabular}

Magnetic declination, $5^{\circ} 30^{\prime} \mathrm{W}$.

From a point 0.5 mile east of Sideburn, south 1 mile, then west.

NoTE.-This line was unfinished; no check azimuth and no closure.

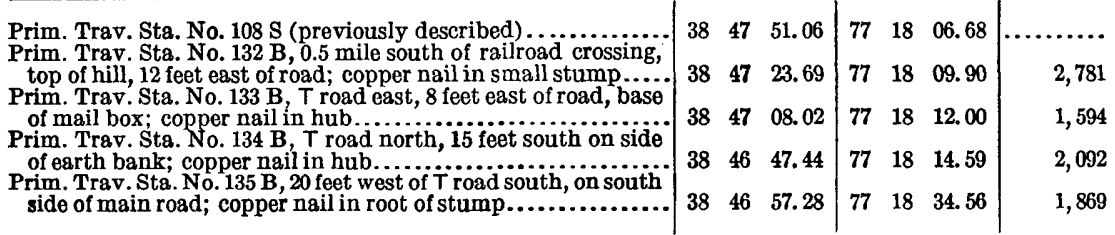

Magnetic declination, $5^{\circ} 30^{\prime} \mathrm{W}$. 
CAPE HENRY QUADRANGLE.

Along highways near Broad Creek. (By F. J. McMaugh in 1917.)

\begin{tabular}{|c|c|c|c|}
\hline Station. & Latitude. & Longitude. & $\begin{array}{l}\text { Distance } \\
\text { between } \\
\text { stations. }\end{array}$ \\
\hline 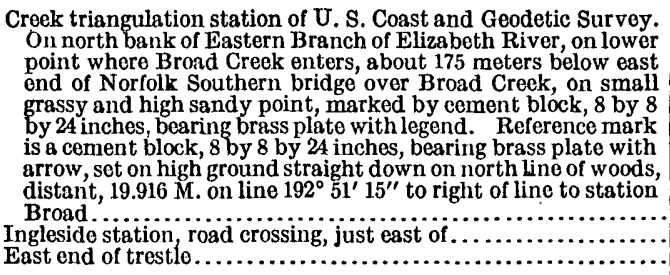 & \begin{tabular}{ccc}
$\circ$ &, & \multicolumn{1}{c}{$\prime$} \\
36 & 50 & 23.76 \\
36 & 50 & 33.6 \\
36 & 50 & 38.6
\end{tabular} & \begin{tabular}{ccl}
$\circ$ & $\prime$ & \multicolumn{1}{c}{} \\
76 & 13 & 46.34 \\
76 & 14 & 05.7 \\
76 & 14 & 25.8
\end{tabular} & $\begin{array}{r}\text { Feet. } \\
\text { 1, } 865 \\
1,705\end{array}$ \\
\hline
\end{tabular}

North along Virginian Ry. near west border of quadrangle.

Tidewater Junction, intersection of Norfolk Southern R. R. with Virginian Ry., south and east rails.

Road crossing .

Road crossing, concrete boulevard to virginia Beach................

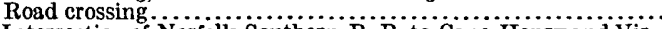

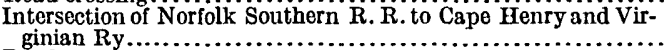

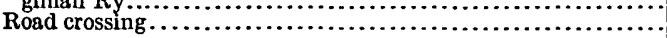

\begin{tabular}{|rrr|rrr|r}
36 & 50 & 41.5 & 76 & 14 & 37.1 & \\
36 & 51 & 02.4 & 76 & 14 & 42.3 & 970 \\
36 & 51 & 12.4 & 76 & 14 & 44.8 & 1,155 \\
36 & 51 & 41.6 & 76 & 14 & 52.0 & 3,009 \\
36 & 51 & 47.8 & 76 & 14 & 53.5 & \\
36 & 52 & 02.2 & 76 & 14 & 57.1 & 1,478 \\
\hline
\end{tabular}

Northeast along Norfolk Southern R. R.

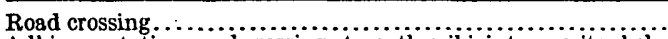

Adkinson station, road crossing at, south rail joint opposite shel-

ter house.

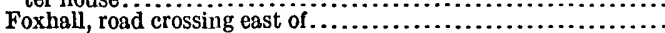

Halstead, switch point.

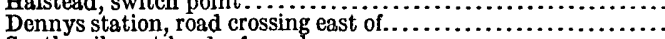

South rail, east bank of canal. . . . . . . . . . .

Rosd crossing.

Steel smoke stack, about 0.6 mile northeast of Burtons station....

Burtons, road crossing at.

Road crossing, northwest of white house with green shutters,

brick chinmey, picket fence to water bank, near point of curvature of railroad

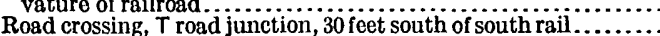

Diamond Springs station, road crossing east of ...............

Lake Smith, 50 feet east of shelter house, 15 feet south of railroad;

Lake Smith, 50 feet east of shelter house, 15 feet south of railroad;

iron post stamped "Prim. Trav. Sta. No. 1, 1917" $\ldots \ldots \ldots \ldots \ldots \ldots \ldots \ldots \ldots \ldots \ldots \ldots \ldots \ldots \ldots \ldots \ldots \ldots \ldots \ldots$
East end of long trestle $\ldots \ldots \ldots \ldots \ldots \ldots$

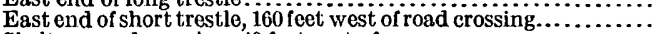

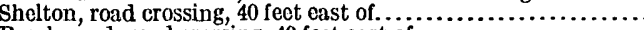

\begin{tabular}{|c|c|c|c|c|c|c|}
\hline 36 & & 55.4 & 76 & 14 & 31.2 & 1,531 \\
\hline $\begin{array}{l}36 \\
36 \\
36 \\
36 \\
36 \\
36 \\
36 \\
36\end{array}$ & $\begin{array}{l}52 \\
52 \\
52 \\
52 \\
52 \\
52 \\
53 \\
53\end{array}$ & $\begin{array}{l}00.5 \\
11.8 \\
27.5 \\
48.8 \\
50.7 \\
57.1 \\
19.4 \\
08.2\end{array}$ & $\begin{array}{l}76 \\
76 \\
76 \\
76 \\
76 \\
76 \\
76 \\
76\end{array}$ & $\begin{array}{l}14 \\
13 \\
13 \\
12 \\
12 \\
12 \\
12 \\
11\end{array}$ & $\begin{array}{l}25.2 \\
58.8 \\
21.8 \\
31.6 \\
27.2 \\
12.0 \\
23.1 \\
46.0\end{array}$ & $\begin{array}{r}1,106 \\
2,429 \\
3,396 \\
4,614 \\
409 \\
1,392 \\
2,432 \\
3,225\end{array}$ \\
\hline $\begin{array}{l}36 \\
36 \\
36 \\
36\end{array}$ & $\begin{array}{l}53 \\
53 \\
53 \\
53\end{array}$ & $\begin{array}{l}19.6 \\
23.7 \\
25.9 \\
31.9\end{array}$ & $\begin{array}{l}76 \\
76 \\
76 \\
76\end{array}$ & $\begin{array}{l}11 \\
11 \\
10 \\
10\end{array}$ & $\begin{array}{l}19.0 \\
04.1 \\
50.8 \\
14.6\end{array}$ & $\begin{array}{l}2,477 \\
1,280 \\
1,100 \\
3,004\end{array}$ \\
\hline $\begin{array}{l}36 \\
36 \\
36 \\
36 \\
36\end{array}$ & $\begin{array}{l}\mathbf{5 3} \\
53 \\
53 \\
\mathbf{5 3} \\
53\end{array}$ & $\begin{array}{l}35.7 \\
38.7 \\
40.6 \\
57.6 \\
57.7\end{array}$ & $\begin{array}{l}76 \\
76 \\
76 \\
76 \\
76\end{array}$ & $\begin{array}{l}09 \\
09 \\
09 \\
08 \\
08\end{array}$ & $\begin{array}{l}53.5 \\
42.8 \\
35.7 \\
47.1 \\
15.5\end{array}$ & $\begin{array}{r}1,753 \\
922 \\
612 \\
4,099 \\
2,638\end{array}$ \\
\hline 36 & 54 & 06.1 & 76 & 07 & 32.7 & 3,581 \\
\hline $\begin{array}{l}36 \\
36 \\
36 \\
36 \\
36 \\
36\end{array}$ & $\begin{array}{l}54 \\
54 \\
54 \\
54 \\
54 \\
54\end{array}$ & $\begin{array}{l}08.4 \\
13.2 \\
26.8 \\
27.4 \\
25.5 \\
35.3\end{array}$ & $\begin{array}{l}76 \\
76 \\
76 \\
76 \\
76 \\
76\end{array}$ & $\begin{array}{l}07 \\
07 \\
06 \\
05 \\
05 \\
04\end{array}$ & $\begin{array}{l}13.5 \\
07.0 \\
25.7 \\
42.2 \\
20.2 \\
26.3\end{array}$ & $\begin{array}{r}1,577 \\
719 \\
3,627 \\
3,531 \\
1,798 \\
4,493\end{array}$ \\
\hline $\begin{array}{l}36 \\
36 \\
36 \\
36 \\
36 \\
36 \\
36\end{array}$ & $\begin{array}{l}54 \\
\mathbf{5 4} \\
\mathbf{5 5} \\
\mathbf{5 5} \\
\mathbf{5 5} \\
\mathbf{5 5} \\
\mathbf{5 5}\end{array}$ & $\begin{array}{l}42.6 \\
53.7 \\
22.6 \\
43.4 \\
39.8 \\
34.3 \\
34.80\end{array}$ & $\begin{array}{l}76 \\
76 \\
76 \\
76 \\
76 \\
76 \\
76\end{array}$ & $\begin{array}{l}03 \\
03 \\
02 \\
01 \\
00 \\
00 \\
00\end{array}$ & $\begin{array}{l}51.7 \\
16.8 \\
24.0 \\
22.4 \\
54.8 \\
36.1 \\
26.93\end{array}$ & $\begin{array}{r}2,906 \\
3,050 \\
5,193 \\
5,425 \\
2,271 \\
1,614 \\
749\end{array}$ \\
\hline 36 & 55 & 35.02 & 76 & 00 & 26.73 & 27 \\
\hline 36 & 54 & 32.8 & 76 & 05 & 57.3 & $\cdots$ \\
\hline 36 & 54 & 09.9 & 76 & 05 & 35.7 & \\
\hline 36 & 54 & 35.5 & 76 & 05 & 05.1 & \\
\hline
\end{tabular}

Beechwood, road crossing, 40 feet east of .....................

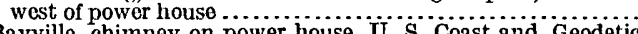

Bayville, chimney on power house, U. S. Coast and Geodetic

Survey triangulation station . ..........................

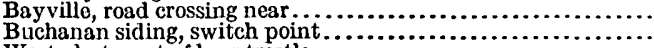

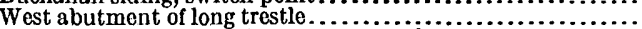

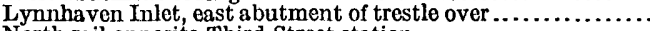

North rail opposite Third Street station.

James Fishery, 20 feet north of railroad, 10 feet west of road cross-

ing; iron post stamped "Prim. Trav. Sta. No. 2, 1917" . .......

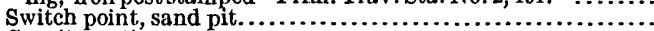

Granite station.

Fifty-second Street station

Porty-fifth Street station

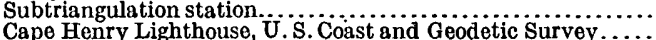

Cape Henry Lighthouse, 10 feet north and 12 feet east of entrance iron post stamped "Prim. Trav. Sta. No. 3, 1917," Elev. 13"

Buchanan siding, about 2,400 feet northeast of, 250 feet north of railroad track, approximately 1,000 feet northeast of red bungalow, west of west end of drawbridge; Coast and Geodetic

Survey tablet set in tile fllled with concrete. (Point A.).....
Lynnhaven Inlet, about 2,000 feet southwest of, low grassy point on west side of inlet, approximately 800 feet south of draw-

bridge. (Point B.)................................................

Lynnhaven Inlet, triangle point about 1,600 feet northeast of

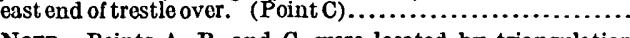

Nore.-Points A, B, and C, were located by triangulation trom traverse line. 
CAPE HENRY QUADRANGIE-Continued.

South along railroad near west border of quadrangle. (By F. J. Mckaugh in 1917.)

\begin{tabular}{|c|c|c|c|c|c|}
\hline Station. & \multicolumn{2}{|c|}{ Latitude. } & \multicolumn{2}{|c|}{ Longitude. } & $\begin{array}{l}\text { Distance } \\
\text { between } \\
\text { stations. }\end{array}$ \\
\hline 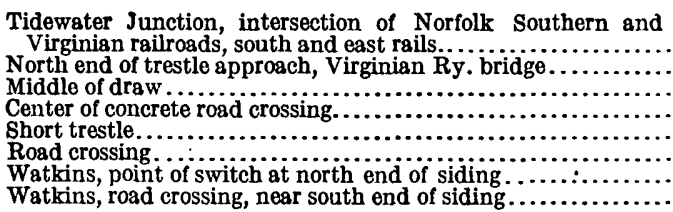 & $\begin{array}{ll}0 & 1 \\
36 & 50 \\
36 & 50 \\
36 & 50 \\
36 & 49 \\
36 & 46 \\
36 & 4 \\
36 & 4 \\
36 & 4\end{array}$ & $\begin{array}{l}41.5 \\
18.6 \\
10.9 \\
43.4 \\
26.0 \\
54.1 \\
30.4 \\
12.3\end{array}$ & $\begin{array}{c}\circ \\
76 \\
76 \\
76 \\
76 \\
76 \\
76 \\
76 \\
76\end{array}$ & $\begin{array}{cc}\prime & \prime \prime \\
14 & 37.1 \\
14 & 36.3 \\
14 & 39.7 \\
14 & 59.6 \\
14 & 52.4 \\
14 & 42.5 \\
14 & 26.9 \\
14 & 07.1\end{array}$ & $\begin{array}{r}\text { Feet. } \\
\ldots 2,312 \\
833 \\
3,220 \\
\ldots \\
3,320 \\
2,713 \\
2,443\end{array}$ \\
\hline
\end{tabular}

East along highways near south border of quadrangle.

Butts, 1.3 miles northeast of, 30 feet west of road, in southeast corner of yard; iron post stamped "Prim. Trav. Sta. No. 6, 1917 "...........................................................

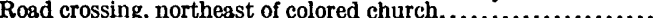

Culvert crossing rosd, white house northeast.

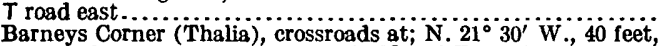
to southeast corner of store porch; N. $55^{\circ}$ E. 33 feet to southwest corner of store building; N. $82^{\circ}$ W., 55 feet, to telephone

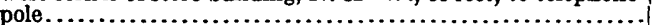

Center of small bridge about 870 feet southeast of another

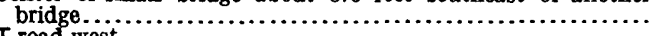

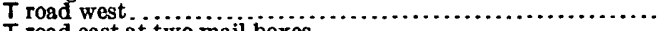

$T$ road east at two mail boxes........... road east, about 500 450 feet southeast of yellow schoolhouse..........................

Road south along bank of reservoir, 190 feet south of pumping

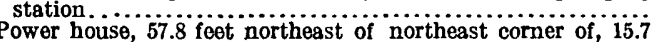
feet from 2 by 6 inch post at edge of road, 54 feet from 3-foot oak tree; iron post stamped "Prim. Trav. Sta. No. 7 " ........

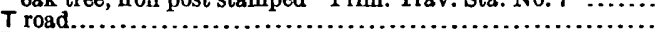
Intersection of roads, $T$ road west, 30 feet southeast to corner of wire fence, 30 feet northwest to two pine trees............

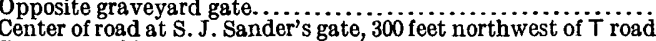
Center of road at S. J. Sander's gate, 300 feet northwest of T road. Senter of bridge . .............................................. road northeast and east edge of road northwest; nail in stake. Intersection of farm road, at angle in main road ................

$T$ road west, at. center of north and south road, 40 feet north of bridge over ditch, 45 feet south of mail box 32,4 feet east

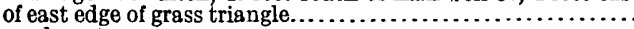
$T$ rosd west

Road fork, 10 feet east of wire fence, 35 feet southwest of pine tree, in center of grass triangle, in line with north edge of

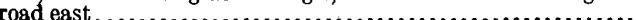

\begin{tabular}{|c|c|c|c|c|c|c|}
\hline $\begin{array}{l}36 \\
36 \\
36 \\
36 \\
36\end{array}$ & $\begin{array}{l}45 \\
46 \\
46 \\
47 \\
47\end{array}$ & $\begin{array}{l}14.1 \\
08.0 \\
49.4 \\
08.8 \\
12.6\end{array}$ & $\begin{array}{l}76 \\
76 \\
76 \\
76 \\
76\end{array}$ & $\begin{array}{l}12 \\
12 \\
11 \\
11 \\
11\end{array}$ & $\begin{array}{l}27.0 \\
15.2 \\
32.7 \\
07.9 \\
04.1\end{array}$ & $\begin{array}{r}5,528 \\
5,436 \\
2,815 \\
490\end{array}$ \\
\hline $\begin{array}{l}36 \\
36\end{array}$ & $\begin{array}{l}47 \\
47\end{array}$ & $\begin{array}{l}50.7 \\
24.4\end{array}$ & $\begin{array}{l}76 \\
76\end{array}$ & $\begin{array}{l}10 \\
09\end{array}$ & $\begin{array}{l}30.7 \\
55.7\end{array}$ & $\begin{array}{l}4,714 \\
3,894\end{array}$ \\
\hline $\begin{array}{l}36 \\
36 \\
36\end{array}$ & $\begin{array}{l}47 \\
46 \\
46\end{array}$ & $\begin{array}{l}00.1 \\
52.4 \\
14.2\end{array}$ & $\begin{array}{l}76 \\
76 \\
76\end{array}$ & $\begin{array}{l}09 \\
09 \\
09\end{array}$ & $\begin{array}{l}32.9 \\
32.2 \\
13.2\end{array}$ & $\begin{array}{r}3,086 \\
778 \\
4,163\end{array}$ \\
\hline 36 & 46 & 01.2 & 76 & 09 & 02.7 & 1,568 \\
\hline 36 & 45 & 37.7 & 76 & 08 & 34.5 & 3,303 \\
\hline $\begin{array}{l}36 \\
36\end{array}$ & $\begin{array}{l}45 \\
45\end{array}$ & $\begin{array}{l}37.2 \\
40.4\end{array}$ & $\begin{array}{l}76 \\
76\end{array}$ & $\begin{array}{l}08 \\
08\end{array}$ & $\begin{array}{l}31.8 \\
26.6\end{array}$ & $\begin{array}{l}223 \\
539\end{array}$ \\
\hline $\begin{array}{l}36 \\
36 \\
36 \\
36\end{array}$ & $\begin{array}{l}46 \\
45 \\
45 \\
45\end{array}$ & $\begin{array}{l}05.8 \\
38.0 \\
33.1 \\
25.4\end{array}$ & $\begin{array}{l}76 \\
76 \\
76 \\
76\end{array}$ & $\begin{array}{l}07 \\
07 \\
06 \\
06\end{array}$ & $\begin{array}{l}25.4 \\
06.4 \\
58.4 \\
42.8\end{array}$ & $\begin{array}{r}5,595 \\
3,207 \\
820 \\
1,490\end{array}$ \\
\hline $\begin{array}{l}36 \\
36\end{array}$ & $\begin{array}{l}45 \\
45\end{array}$ & $\begin{array}{l}14.8 \\
24.3\end{array}$ & $\begin{array}{l}76 \\
76\end{array}$ & $\begin{array}{l}06 \\
05\end{array}$ & $\begin{array}{l}30.7 \\
57.2\end{array}$ & $\begin{array}{l}1,457 \\
2,894\end{array}$ \\
\hline $\begin{array}{l}36 \\
36\end{array}$ & $\begin{array}{l}45 \\
45\end{array}$ & $\begin{array}{l}35.6 \\
55.6\end{array}$ & $\begin{array}{l}76 \\
76\end{array}$ & $\begin{array}{l}05 \\
05\end{array}$ & $\begin{array}{l}32.9 \\
36.4\end{array}$ & $\begin{array}{l}2,279 \\
2,048\end{array}$ \\
\hline 36 & 46 & 26.8 & 76 & 05 & 41.9 & 3,182 \\
\hline
\end{tabular}

Southeast along Norfolk Southern R. R. (Currituck Branch).

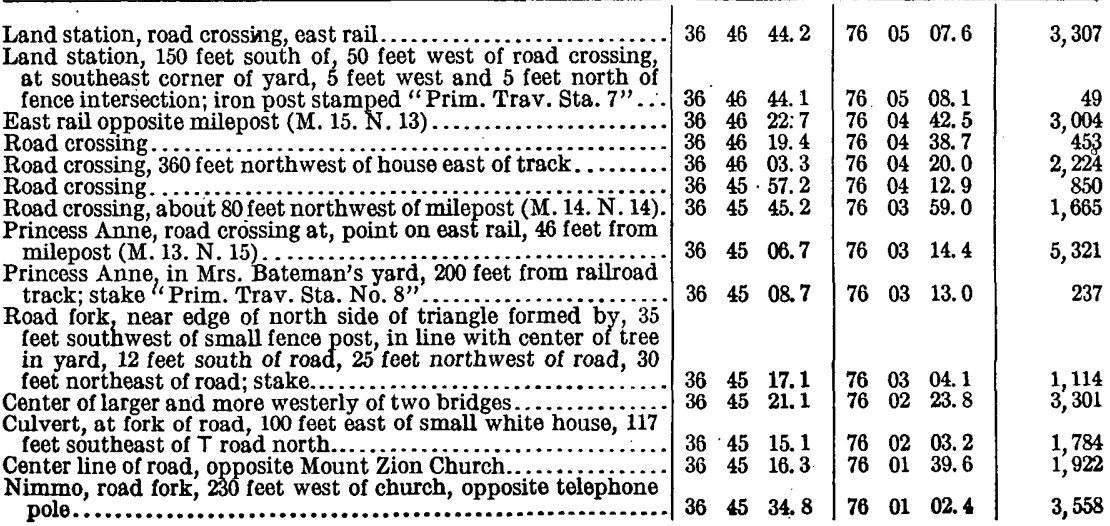


CAPE HENRY QUADRANGIE-Continued.

Southeast along Norfolk Southern R. R.-Continued.

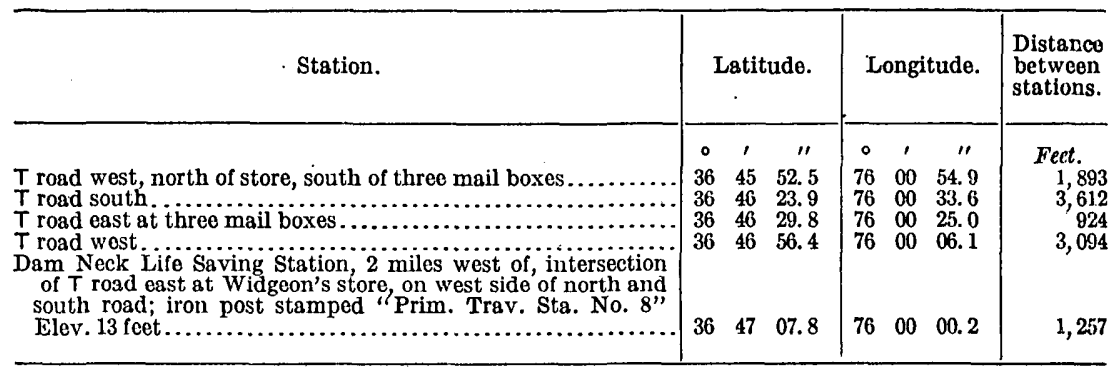

Magnetic declination for quadrangle, $5.6^{\circ} \mathrm{W}$.

South on Ocean View electric rallroad.

\begin{tabular}{|c|c|c|c|c|c|c|}
\hline Road crossing $\ldots \ldots \ldots \ldots \ldots \ldots \ldots \ldots \ldots \ldots$ & 36 & 06.3 & 76 & 14 & 55.4 & \\
\hline Road crossing near railroad station.............. & 36 & 34.7 & 76 & 14 & 46.0 & 3,285 \\
\hline 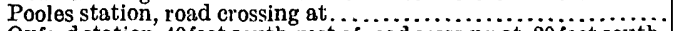 & 36 & 46.5 & 76 & 14 & 38.6 & $4,9.12$ \\
\hline $\begin{array}{l}\text { Oxford station, 40 feet southwest of road crossing at, } 20 \text { feet south } \\
\text { of road west; iron post stamped "Prim. Trav. Sta. No. 5, 1917". }\end{array}$ & 36 & 52.6 & 76 & 14 & 30.8 & 5,487 \\
\hline
\end{tabular}

East and south along highways to Camden Helghts.

Metal culvert, center of road. .

$T$ road north, center of.

$T$ road north at bend in road.

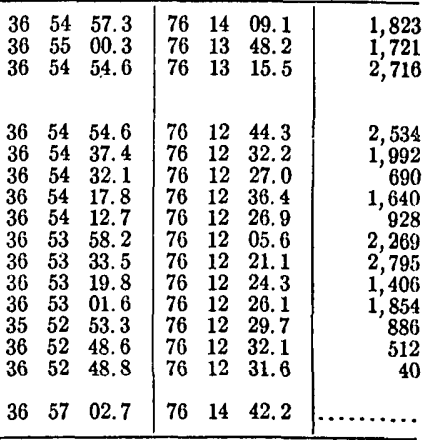

$x$ ford station, 1.5 miles east of, crossroads at Water's store, 18

feet southeast to southwest corner and 20 feet northeast to

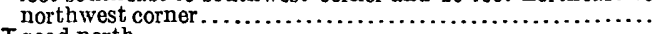

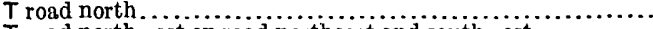

$T$ road northwest on road northeast and southwest...........

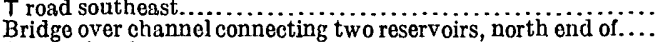

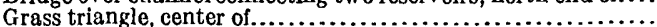

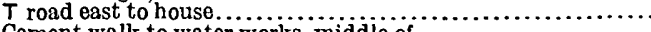

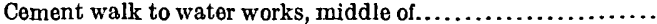

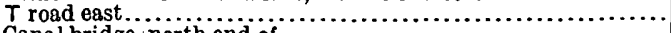

Canal bridge, north end of.

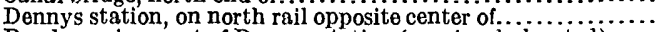

Road crossing, east of Dennys station (previously located)......

Triangulation station near shore line, Ocean View (C. \& G. S.

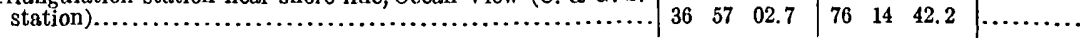

Magnetic declination for quadrangle, $5.5^{\circ} \mathrm{W}$.

CHARLES CITY QUADRANGLE.

South along Chesapeake \& Ohio Ry. and highways near east borḍer of quadrangle. (By J. H. Wilson in 1916.)

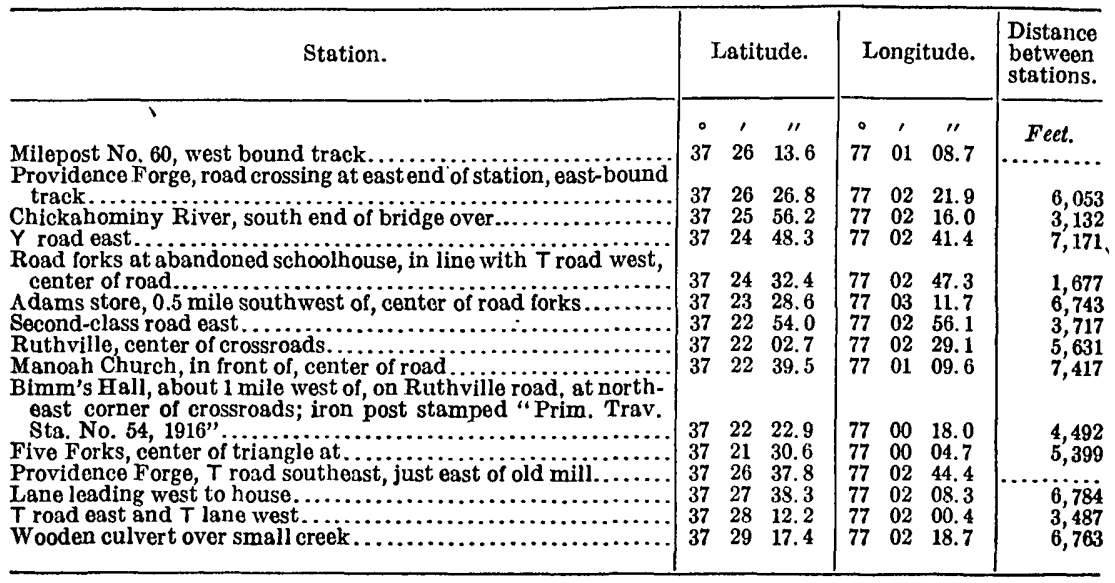


CHARLES CITY QUADRANGLE-Continued.

West along highways near center of quadrangle.

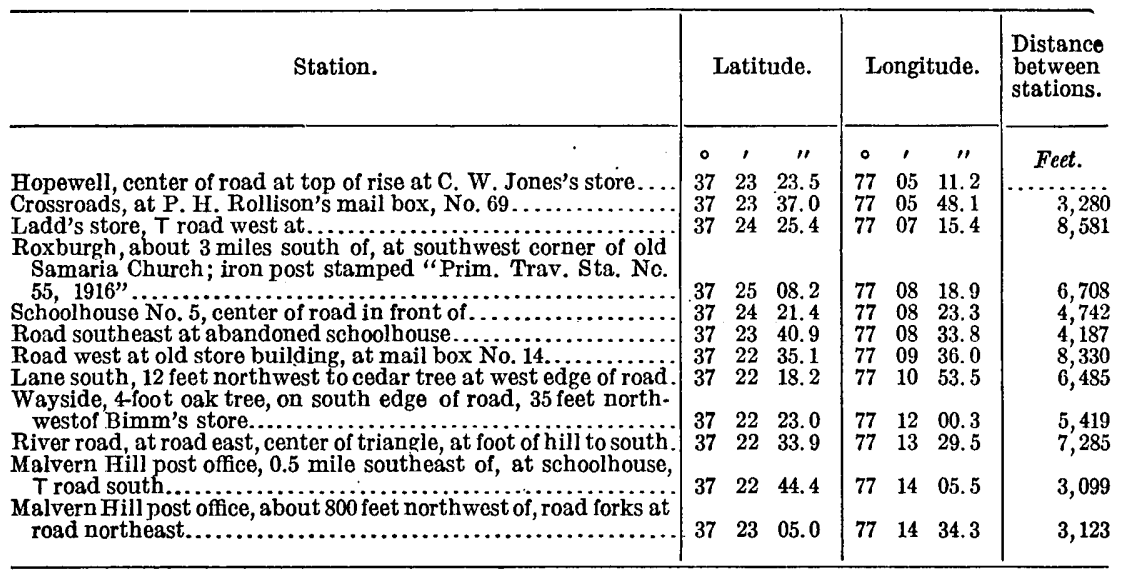

North along highways near west border of quadrangle.

\begin{tabular}{|c|c|c|c|c|c|c|c|}
\hline 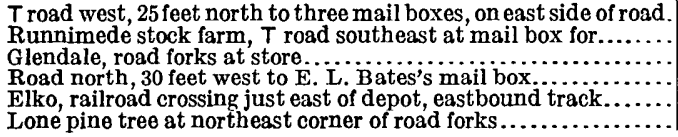 & $\begin{array}{l}37 \\
37 \\
37 \\
37 \\
37 \\
37\end{array}$ & $\begin{array}{l}24 \\
25 \\
26 \\
27 \\
28 \\
29\end{array}$ & $\begin{array}{l}37.2 \\
33.5 \\
45.4 \\
04.9 \\
21.0 \\
05.2\end{array}$ & $\begin{array}{l}77 \\
77 \\
77 \\
77 \\
77 \\
77\end{array}$ & $\begin{array}{l}14 \\
14 \\
13 \\
12 \\
12 \\
12\end{array}$ & $\begin{array}{l}56.9 \\
27.7 \\
50.8 \\
48.9 \\
44.2 \\
45.3\end{array}$ & $\begin{array}{r}6,161 \\
7,860 \\
5,370 \\
7,705 \\
4,480\end{array}$ \\
\hline
\end{tabular}

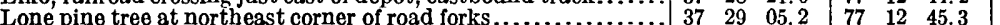

Magnetic declination for east border of quadrangle, $5^{\circ} 0 \mathrm{I}^{\prime} \mathrm{W}$.; for west border of quadrangle, $4^{\circ} 37^{\prime} \mathrm{W}$.; near center of quadrangle, $6^{\circ} 18^{\prime} \mathrm{W}$.

(By J. H. Wilson in 1916-17.)

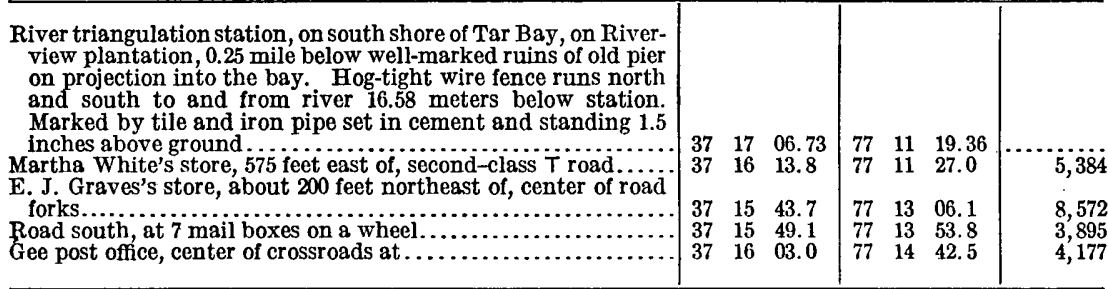

Magnetic declination near southwest corner of quadrangle, $4^{\circ} 33^{\prime} \mathrm{W}$.

Northwest along highways near south border of quadrangle. (By Olinus Smith in 1917.)

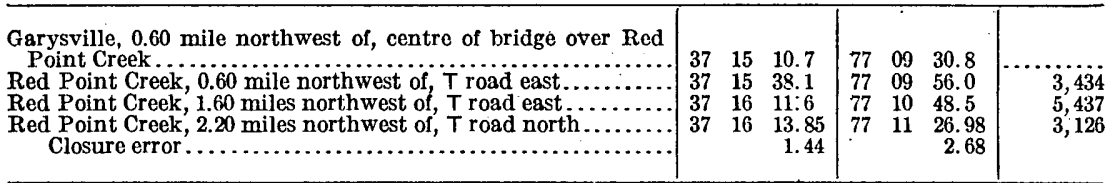

Magnetic declination near south border of quadrangle, $5^{\circ} 50^{\prime} \mathrm{W}$. 
DINWIDDIE QUADRANGIE.

East along Seaboard Air Line R. R. near south border of quadrangle. (By J. C. Fales In 1918.)

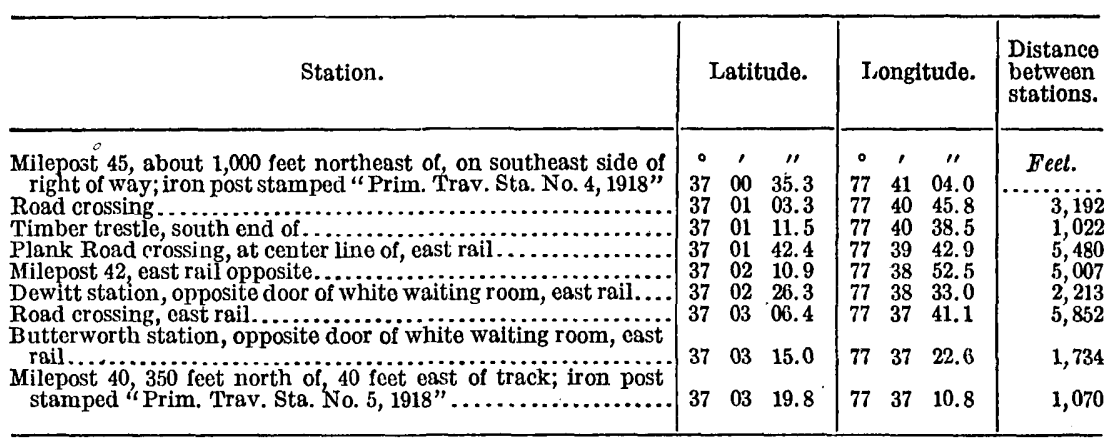

Along logging rallroad near south border of quadrangle.

\begin{tabular}{|c|c|c|c|c|c|c|c|}
\hline 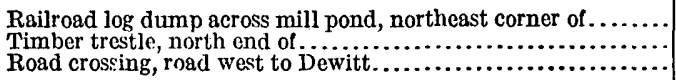 & $\begin{array}{l}37 \\
37 \\
37\end{array}$ & $\begin{array}{l}02 \\
02 \\
01\end{array}$ & $\begin{array}{l}59.1 \\
13.3 \\
47.4\end{array}$ & $\begin{array}{l}77 \\
77 \\
77\end{array}$ & $\begin{array}{l}37 \\
36 \\
36\end{array}$ & $\begin{array}{l}13.4 \\
45.2 \\
27.5\end{array}$ & $\begin{array}{l}2,110 \\
5,163 \\
2,989\end{array}$ \\
\hline
\end{tabular}

Along highways near south border of quadrangle.

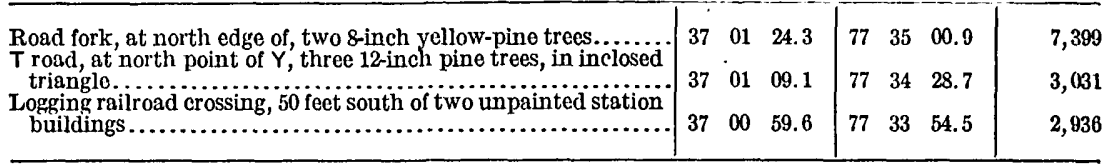

Along logging ratlroad near south border of quadrangle.

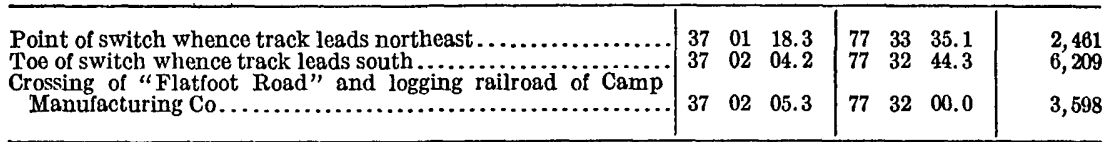

Along highways near south border of quadrangle.

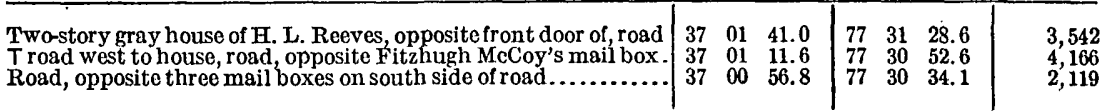

Magnetic declination for south part of quadrangle, $4^{\circ} 10^{\prime} \mathrm{W}$.

DISPUTANTA QUADRANGLE.

In northwest corner of quadrangle. (By J. H. Wilson in 1916-17.)

\begin{tabular}{|c|c|c|c|}
\hline Station. & Latitude. & Longitude. & $\begin{array}{l}\text { Distance } \\
\text { between } \\
\text { stations. }\end{array}$ \\
\hline $\begin{array}{l}\text { Gee post office, } 1.4 \text { miles southeast of, at road north, } 40 \text { feet north- } \\
\text { east of J. Krumel's mail box on north side of road............ } \\
\text { Prince George courthouse, about } 3 \text { miles northeast of, } 36 \text { feet west } \\
\text { of northwest corner of B. M. Harris's store, at road forks, at foot } \\
\text { of mail box; iron post stamped "Prim. Trav. Sta. No. } 57,1917 \text { " }\end{array}$ & $\begin{array}{ccc}0 & \prime & \prime \prime \\
37 & 14 & 57.5 \\
37 & 14 & 28.8\end{array}$ & $\begin{array}{ccc}\circ 7 & 1 & \\
77 & 14 & 14.4 \\
77 & 14 & 51.5\end{array}$ & Feet. \\
\hline
\end{tabular}


DISPUTANTA QUADRANGLE-Continued.

Southeast along Norfolk \& Western Ry. in south part of quadrangle.

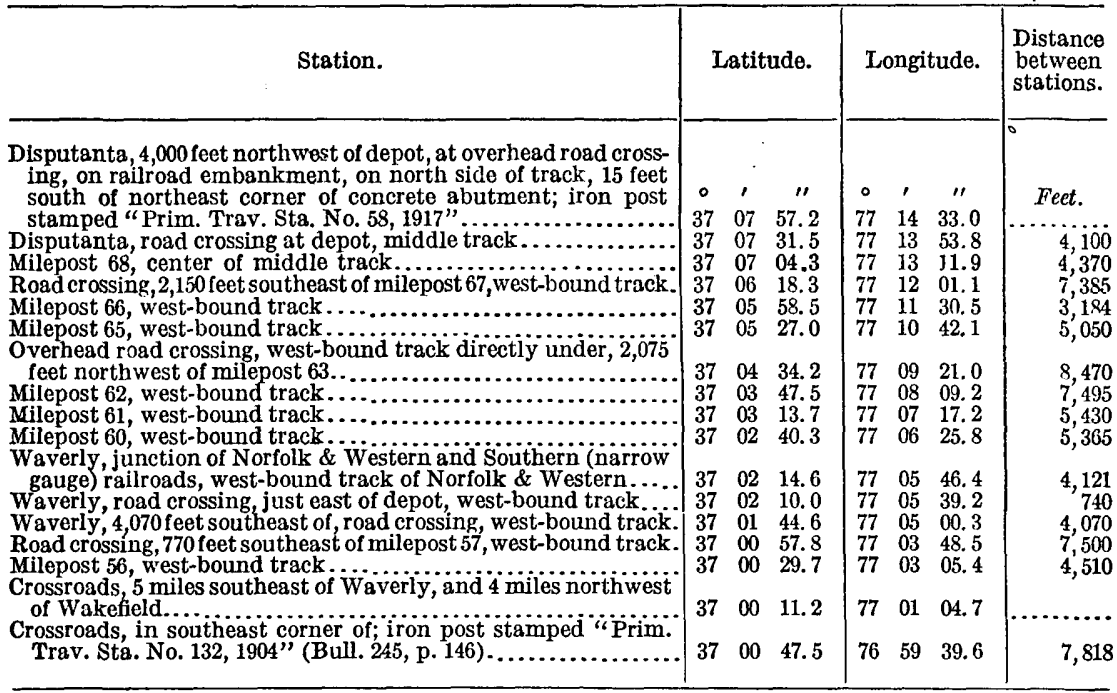

West along Southern Ry. (narrow gauge) and highways in south part of quadrangle.

\begin{tabular}{|c|c|c|c|c|c|c|c|}
\hline Waverly junction... & 37 & 02 & 14. 57 & 77 & 05 & 46. 35 & \\
\hline $\begin{array}{l}\text { Waverly, } 1 \text { mile southwest of at east-west road crossing, } 420 \text { reet } \\
\text { northeast of sign reading "Railroad crossing } 1 \text { mile" }\end{array}$ & 37 & 01 & 36.7 & 77 & 06 & 23.7 & \\
\hline Road crossing, 1,125 feet $n$ or theast of milepost $31 \ldots \ldots \ldots \ldots \ldots$ & 37 & 00 & 56.6 & 77 & 07 & 03.2 & 5,17 \\
\hline Newville Church, 550 feet west of crossroads..... & 37 & 00 & 45.8 & 77 & 10 & 37.7 & \\
\hline Neblet's residence........... & 37 & 00 & 11.9 & 77 & 12 & 36.8 & \\
\hline J & 37 & 00 & 02.4 & 77 & 13 & 04.9 & \\
\hline
\end{tabular}

North along highways near west border of quadrangle.

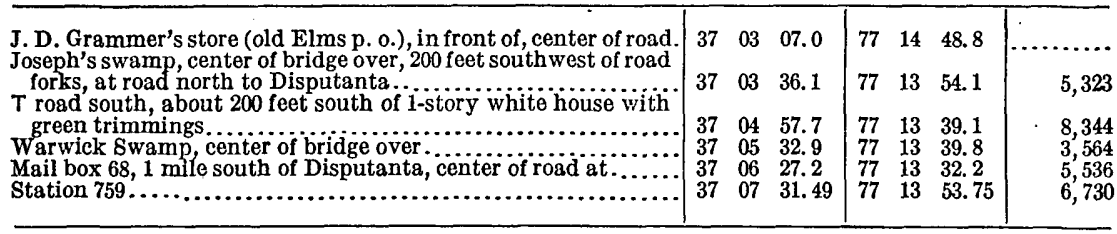

Magnetic declination near northwest corner of quadrangle, $4^{\circ} 02^{\prime} \mathrm{W}$. ; near south border of quadrangle, $2^{\circ} 12^{\prime} \mathrm{W}$.; near west border of quadrangle, $4^{\circ} 11^{\prime} \mathrm{W}$.

North along highways through center of quadrangle. (By Olinus Smith in 1917.)

Waverly, 4.50 miles west of, at Milton siding, under overhead road crossing on Norfolk \& Western Ry., center of west-bound track and Cabin Point roads, crossroads...

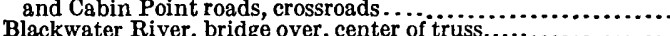

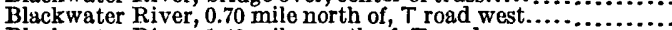

Blackwater River, 1.40 miles north of, $T$ road. . . . . . . . . . . . .

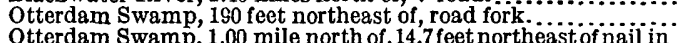

Otterdam Swamp, 1.00 mile north of, 14.7 feet northeast of nail in

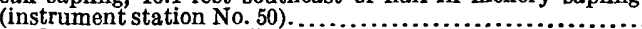

Otterdam Swamp, 1.40 miles north of, at branch crossing, center

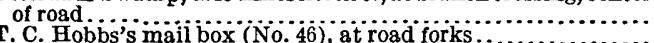

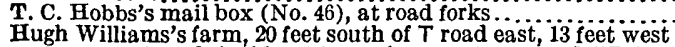
of center of road, inside of fence; iron post stamped "Prim.

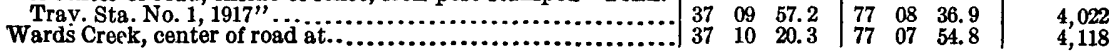


DISPUTANTA QUADRANGLE-Continued.

North along highways through center of quadrangle. (By Olinus Smith in 1917.)-Continued.

\begin{tabular}{|c|c|c|c|c|c|c|c|}
\hline Station. & & Lat & tude. & & ong & itude. & $\begin{array}{l}\text { Distance } \\
\text { between } \\
\text { stations. }\end{array}$ \\
\hline 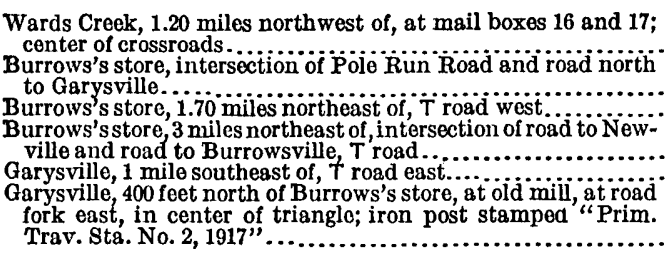 & $\begin{array}{c}\circ \\
37 \\
\\
37 \\
37\end{array}$ & ' & $\begin{array}{r}11.0 \\
07.2 \\
38.8 \\
26.8 \\
27.4 \\
53.7\end{array}$ & $\begin{array}{l}0 \\
77 \\
77 \\
77 \\
77 \\
77 \\
77\end{array}$ & $\begin{array}{l}08 \\
08 \\
07 \\
08\end{array}$ & $\begin{array}{r}\prime \prime \\
13.1 \\
51.5 \\
38.2 \\
56.7 \\
19.3 \\
\\
01.8\end{array}$ & $\begin{array}{r}\text { Fect. } \\
5,340 \\
3,139 \\
9,313 \\
5,892 \\
6,395 \\
\\
4,345\end{array}$ \\
\hline
\end{tabular}

Magnetic declination through center of quadrangle north-south, $5^{\circ} 50^{\prime} \mathrm{W}$.

DOSWELL QUADRANGLE.

East along highways near south border of quadrangle. (By E. L. McNair in 1916.)

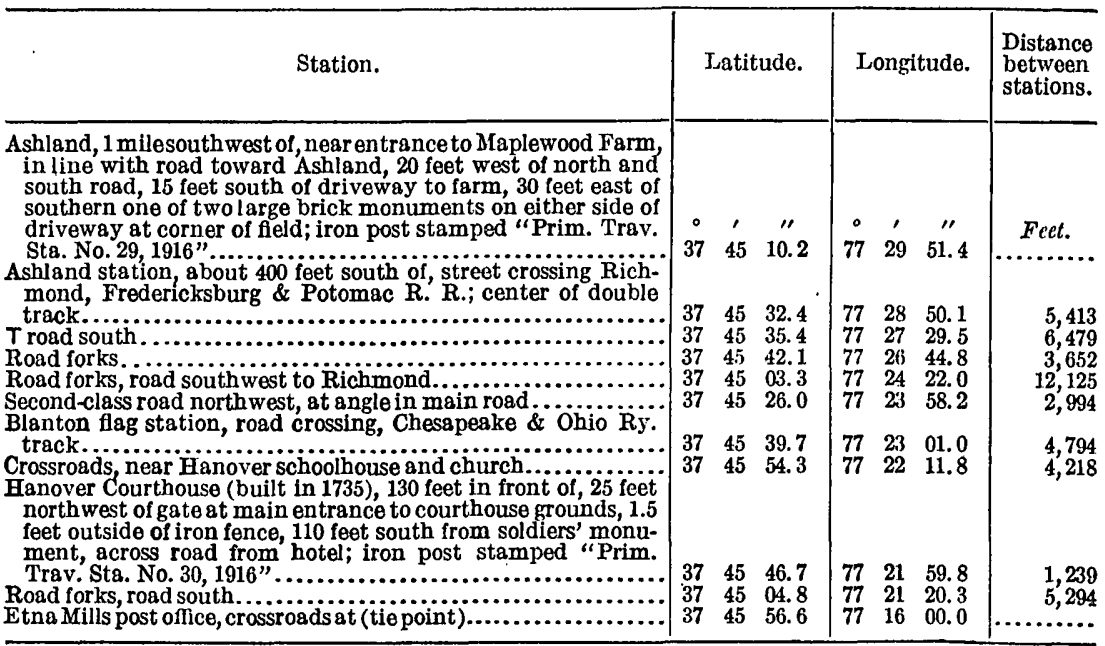

Along highways diagonally east to west through south part of north half of quadrangle.

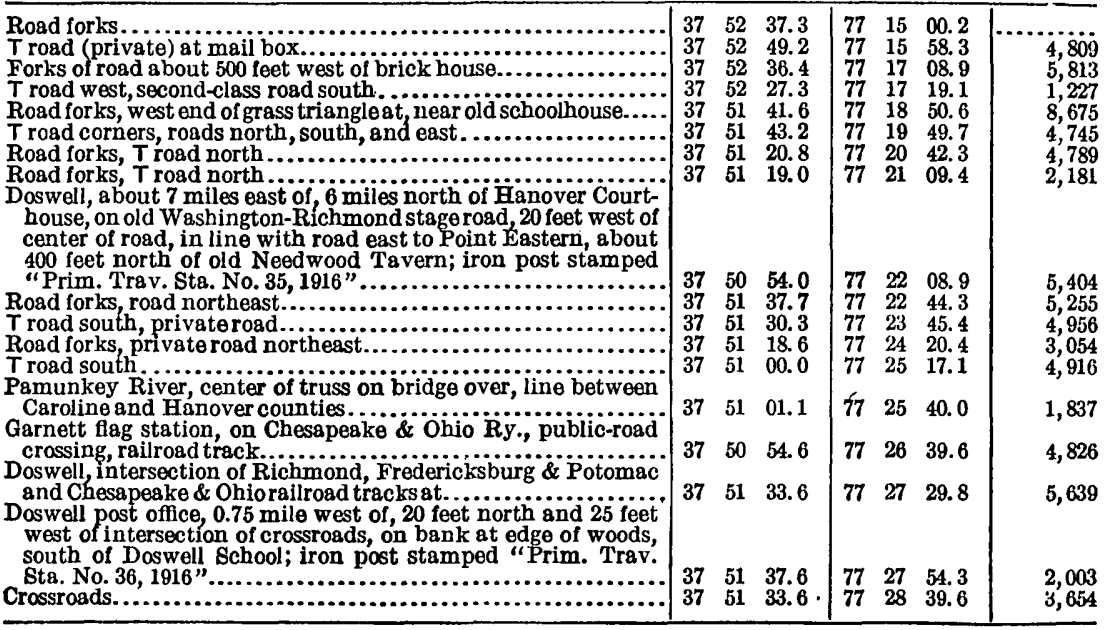


DOSWELL QUADRANGLE-Continued.

Along highways near west border in southwest corner of quadrangle.

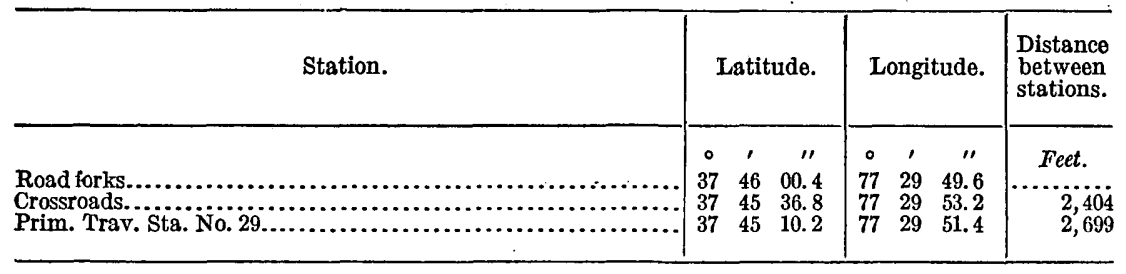

Along highways near east border of quadrangle (south two-thirds of quadrangle).

Primary Traverse Station No. 34 .

Road forks.

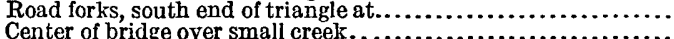

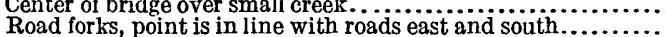

Troad northwest.

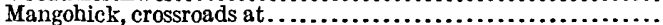

Road forks 20 feet west of oak tree at north end of triangle

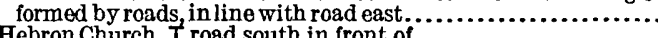

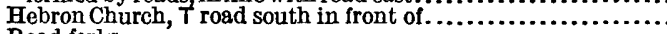

Road forks.

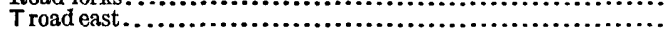

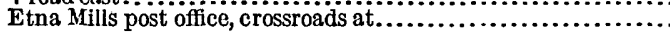

\begin{tabular}{|lll|lll|l}
37 & 52 & 01.1 & 77 & 14 & 49.0 & $\ldots \ldots \ldots \ldots$ \\
37 & 50 & 59.4 & .77 & 15 & 46.0 & $\ldots \ldots \ldots \ldots$ \\
37 & 50 & 47.0 & 77 & 15 & 31.0 & 1,730 \\
37 & 50 & 27.9 & 77 & 15 & 24.4 & 1,998 \\
37 & 49 & 49.8 & 77 & 15 & 32.7 & 3,901 \\
37 & 49 & 38.8 & 77 & 15 & 20.9 & 1,436 \\
37 & 49 & 05.2 & 77 & 16 & 00.2 & 4,633 \\
37 & 48 & 35.6 & 77 & 16 & 24.9 & 3,580 \\
37 & 48 & 30.4 & 77 & 15 & 49.5 & 2,888 \\
37 & 48 & 01.2 & 77 & 15 & 38.6 & 3,069 \\
37 & 47 & 40.0 & 77 & 15 & 26.2 & 2,368 \\
37 & 46 & 52.1 & 77 & 15 & 43.9 & 5,039 \\
37 & 45 & 56.6 & 77 & 16 & 00.0 & 5,754
\end{tabular}

Magnetic declination for south border of quadrangle, $5^{\circ} 05^{\prime} \mathrm{W}$.; for south half of quadrangle, $5^{\circ} 10^{\prime} \mathrm{W}$.; for southwest corner of triangle, $5^{\circ} 00^{\prime} \mathrm{W} . ;$ for east border (south two-thirds) of quadrangle, $4^{\circ} 50^{\prime} \mathrm{W}$.

North along highways and rallroad near west border of north half of quadrangle.

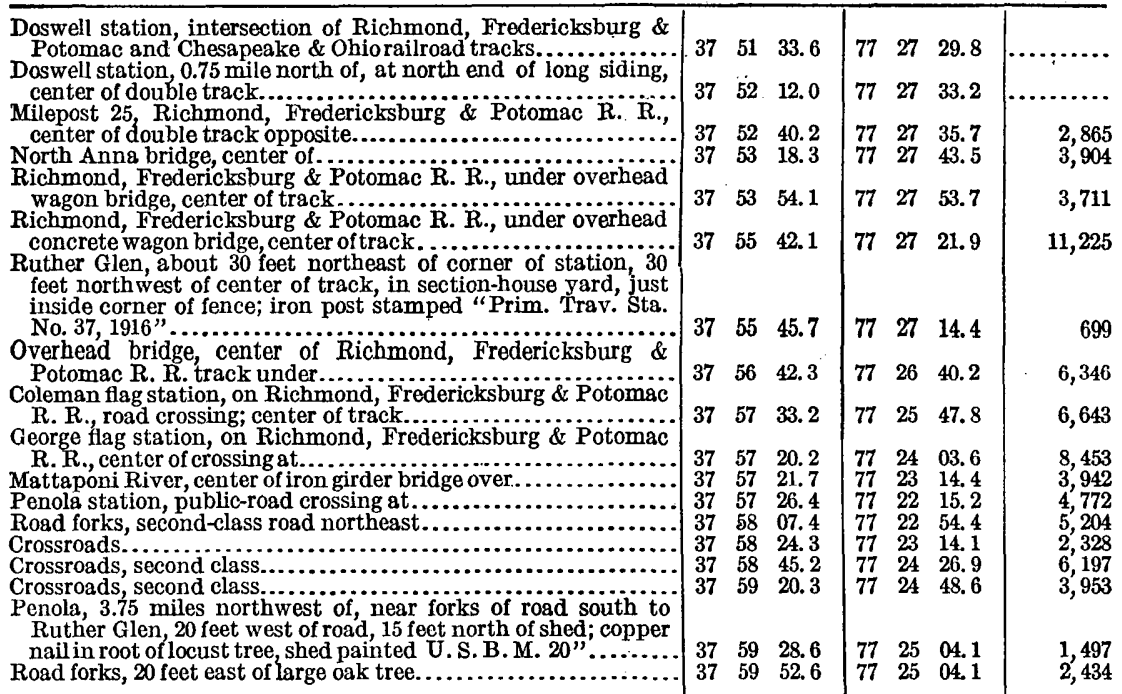

Along highways near north border of quadrangle (spur line).

Balty post office and store, 50 feet southwest of, 50 feet west of road southeast, 20 feet north of east and west road, on top of road southeast, 20 feet north of east and west road, on top, of

Along highways near north border of quadrangle (main line).

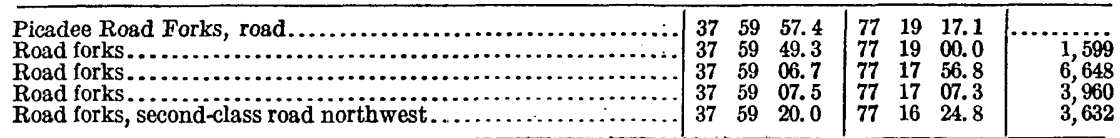

Magnetic declination near west border of quadrangle, $5^{\circ} 20^{\prime} \mathrm{W}$.; near north border of quadrangle, $5^{\circ} 20^{\prime} \mathrm{W}$. 
HOLLAND QUADRANGIE.

Northwest along Virginian Ry. across northeast corner of quadrangle. (By J. J. Charters In 1918.)

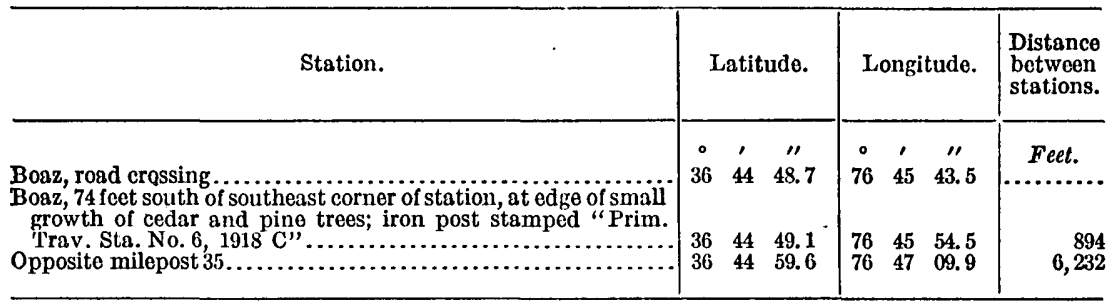

South along highways near east border of quadrangle. (By J. J. Phelan in 1918.)

Road crossing, 100 feet cast of milepost 28 , south rail ...............

Hoad crossing, south rail ................................. west of center of road ................................... Junction of Drumhill and Holland and Franklin roads, north

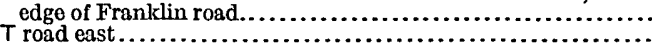

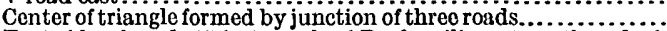

East side of road, 15 feet north of Bush railing at north end of of bridge, 5 feet from edge of approach to bridge...............

South corner of square formed by intersection of roads, 10 feet from corner fence post................................. Center of junction of three roads from Holy Neck, Holland, and

Holly Neck School, 340 ieet east of first $T$ road east of 6 feet from well at Mr. Hayes's house; iron post stamped "Prim.

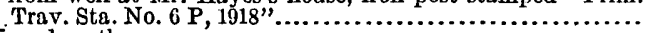
Troad north

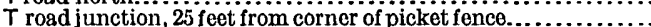

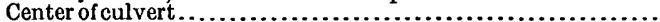

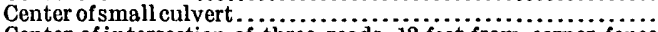

Center of intersection of three roads, 12 feet from corner fence

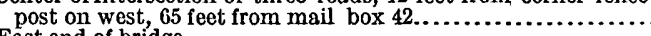

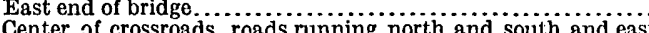

Center of crossroads, roads running north and south and east

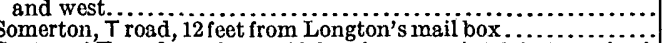

Center of $T$ road southeast, 30 feet from a point 6 feet north of mail box 26.

\begin{tabular}{|rrr|rrr|r}
36 & 41 & 10.0 & 76 & 45 & 14.5 & $\ldots \ldots, 6$ \\
36 & 40 & 53.4 & 76 & 46 & 33.1 & $\mathbf{6 , 6 1 9}$ \\
36 & 40 & 49.2 & 76 & 46 & 53.0 & 1,671 \\
36 & 40 & 26.6 & 76 & 47 & 11.5 & 2,750 \\
36 & 38 & 33.7 & 76 & 47 & 03.4 & 11,442 \\
36 & 38 & 25.2 & 76 & 47 & 01.2 & 882 \\
36 & 37 & 54.5 & 76 & 46 & 37.8 & 3,655 \\
36 & 37 & 35.4 & 76 & 46 & 20.0 & 2,410 \\
& & & & & \\
36 & 36 & 45.6 & 76 & 46 & 51.2 & 5,646 \\
& & & & & & \\
36 & 36 & 47.6 & 76 & 46 & 47.6 & 340 \\
36 & 36 & 54.2 & 76 & 45 & 31.3 & 6,253 \\
36 & 36 & 55.1 & 76 & 45 & 20.5 & 874 \\
36 & 36 & 31.9 & 76 & 45 & 11.9 & 2,447 \\
36 & 35 & 43.2 & 76 & 45 & 16.0 & 4,945 \\
36 & 35 & 10.9 & 76 & 45 & 39.7 & 3,802 \\
36 & 34 & 36.6 & 76 & 45 & 58.4 & 3,797 \\
36 & 34 & 11.2 & 76 & 45 & 58.9 & 2,575 \\
36 & 34 & 06.9 & 76 & 45 & 04.2 & 4,464 \\
36 & 33 & 36.5 & 76 & 45 & 04.1 & 3,080 \\
\hline
\end{tabular}

West along Southern Ry.

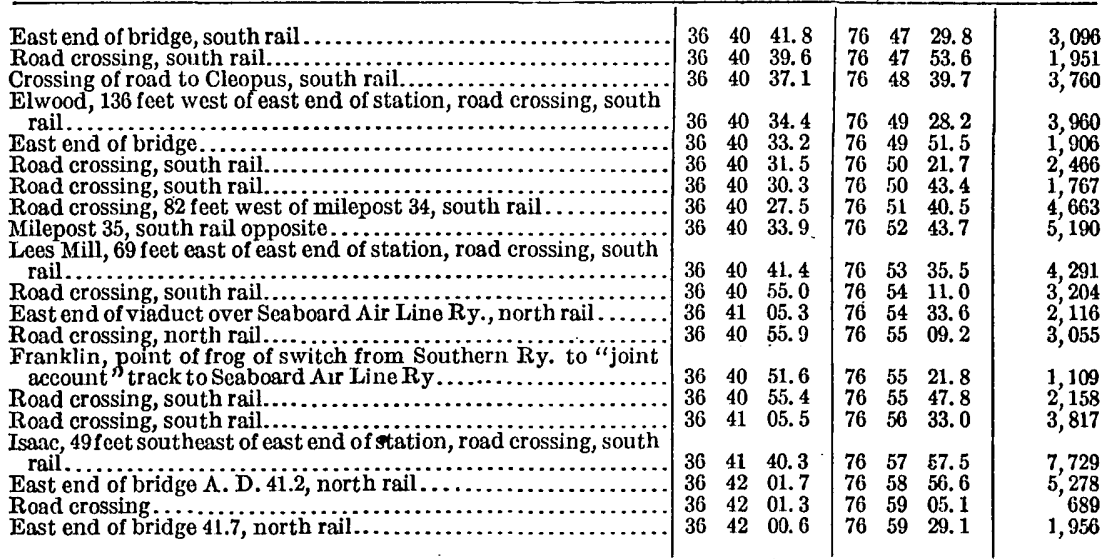

Magnetic declination for east border of quadrangle, $4.3^{\circ} \mathrm{W}$. 
HOLLAND QUADRANGLE-Continued.

Northeast along Seaboard Air Line Ry. (By J. J. Phelan in 1918.)

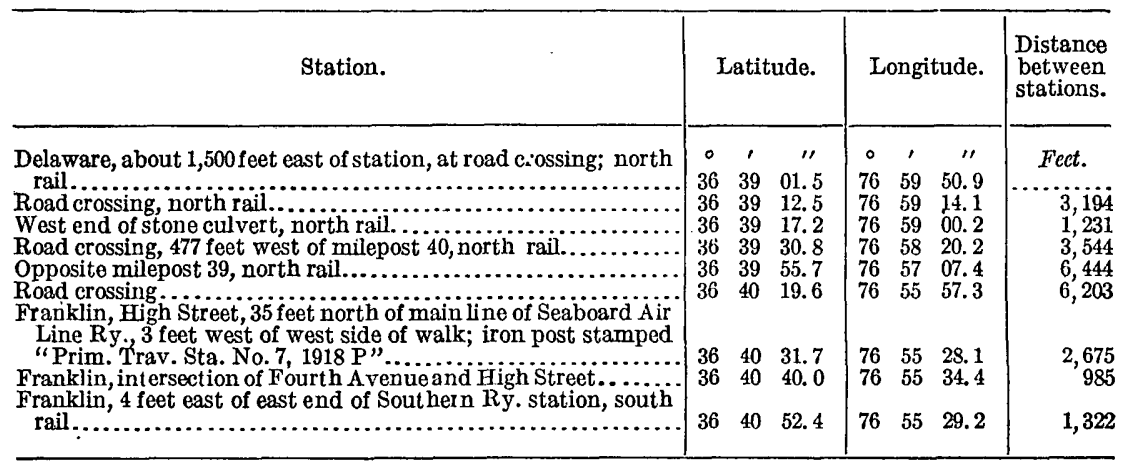

South along highways near center of quadrangle.

\begin{tabular}{|c|c|c|c|c|c|c|c|}
\hline $\begin{array}{l}\text { Elwood, center of road crossing at, south rail..................... } \\
\text { Crossroads, } 10 \text { feet northwest from corner fence post, on edge of }\end{array}$ & & & & 76 & 49 & 28.2 & \\
\hline 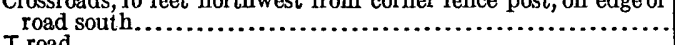 & 36 & 40 & & 76 & 49 & 15.7 & 2,014 \\
\hline $\begin{array}{l}\text { Troad. } \\
\text { Road fork, center of Holland-Franklin road, in line with road to }\end{array}$ & 36 & 39 & & 76 & 49 & 49.7 & 4,22 \\
\hline $\begin{array}{l}\text { Elwood... } \\
\text { Crossroads, Franklin-Holy Neck and Holland-Murfreesboro }\end{array}$ & 36 & 39 & 20.2 & 76 & 50 & 27.2 & 3,997 \\
\hline 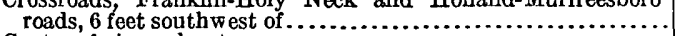 & 36 & 39 & 04.9 & 76 & 51 & 01.4 & 3,200 \\
\hline Center of pipe culvert............................ & 36 & 38 & 42.9 & 76 & 51 & 26.4 & 3,020 \\
\hline 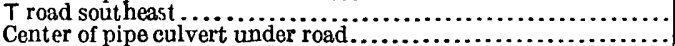 & $\begin{array}{l}36 \\
36\end{array}$ & $\begin{array}{l}38 \\
38\end{array}$ & $\begin{array}{l}26.7 \\
07.1\end{array}$ & $\begin{array}{l}76 \\
76\end{array}$ & $\begin{array}{l}51 \\
52\end{array}$ & $\begin{array}{l}47.4 \\
04.6\end{array}$ & $\begin{array}{l}2,372 \\
2,430\end{array}$ \\
\hline Troad & 36 & 37 & 07.1 & 76 & 52 & 10.6 & 6,083 \\
\hline $\begin{array}{l}\text { Angie } 1 \\
\text { Suffo }\end{array}$ & 36 & & & 76 & & & 68 \\
\hline li culvert at edge of $f$ & 36 & 36 & & 76 & 51 & 58.4 & 9. \\
\hline Tro & 36 & 35 & 53.8 & 76 & 50 & 20.1 & 8,63 \\
\hline ll culvert, near a small sawmill which con- & 36 . & 35 & & 76 & 50 & 06.1 & 1,25 \\
\hline 2,392 feet south of, west side of road, across from $T$ road & 36 & 35 & 38.4 & 76 & 49 & 53.4 & 1,550 \\
\hline & 36 & & & 76 & & & 2,3 \\
\hline Cross & 36 & 35 & 12 & 76 & 49 & & 850 \\
\hline $\begin{array}{l}\text { Culver } \\
\text { Center }\end{array}$ & 36 & 34 & & 76 & 50 & & 3,950 \\
\hline & 36 & 34 & & 76 & 50 & & \\
\hline $\begin{array}{l}\text { Crossroads, } 18 \text { feet fr om Howell I } \\
\text { South edge of road, opposite east }\end{array}$ & 36 & 34 & 17 & 76 & 51 & & \\
\hline South edge of road, opposite east en & 36 & 33 & 24.5 & 76 & 51 & 41.4 & 5,374 \\
\hline
\end{tabular}

North along highways near west border of quadrangle.

\begin{tabular}{|c|c|c|c|c|c|c|c|}
\hline 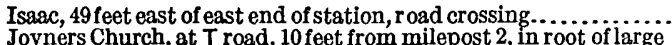 & 36 & 41 & 40.3 & 76 & 57 & 57.5 & \\
\hline 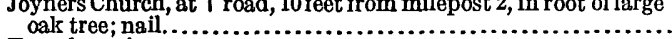 & 36 & 42 & 05.9 & 76 & 57 & 42.0 & 2,886 \\
\hline T road nor theast. . & 36 & 42 & 49. 0 & 76 & 57 & 59.4 & 4,583 \\
\hline 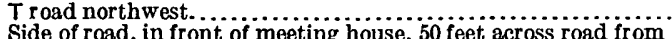 & 36 & 43 & 02.0 & 76 & 57 & 58.6 & 1,323 \\
\hline $\begin{array}{l}\text { Side of road, in front of meeting house, } 50 \text { feet across road from } \\
\text { school } ., \ldots \ldots \ldots \\
\end{array}$ & 36 & 43 & 21,1 & 76 & 57 & 53.8 & 1.975 \\
\hline Center of crossroads.................. & 36 & 43 & 54.4 & 76 & 57 & 16.8 & 4,519 \\
\hline $\begin{array}{l}\text { T road east ... } \\
\text { Center of small bridge, directiy over center pier and } 18 \text { feet from }\end{array}$ & 36 & 44 & 12.4 & 76 & 56 & 44.0 & 3,230 \\
\hline 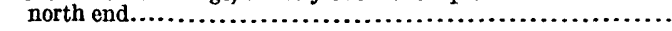 & 36 & 44 & 28.5 & 76 & 56 & 18.8 & 2,617 \\
\hline
\end{tabular}

Southwest along highways from Delaware to Sunbeam.

\begin{tabular}{|c|c|c|c|c|c|c|c|}
\hline 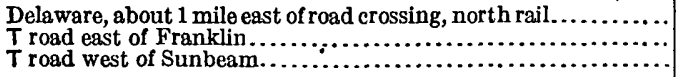 & $\begin{array}{l}36 \\
36 \\
36\end{array}$ & $\begin{array}{l}39 \\
38 \\
37\end{array}$ & $\begin{array}{l}12.5 \\
05.3 \\
56.6\end{array}$ & $\begin{array}{l}76 \\
76 \\
76\end{array}$ & $\begin{array}{l}59 \\
58 \\
58\end{array}$ & $\begin{array}{l}14.1 \\
58.4 \\
56.2\end{array}$ & $\begin{array}{r}6, \underset{994}{900} \\
\quad \cdots\end{array}$ \\
\hline
\end{tabular}


HOLLAND QUADRANGLE-Continued.

Southwest along highways from Delaware to Sanderson.

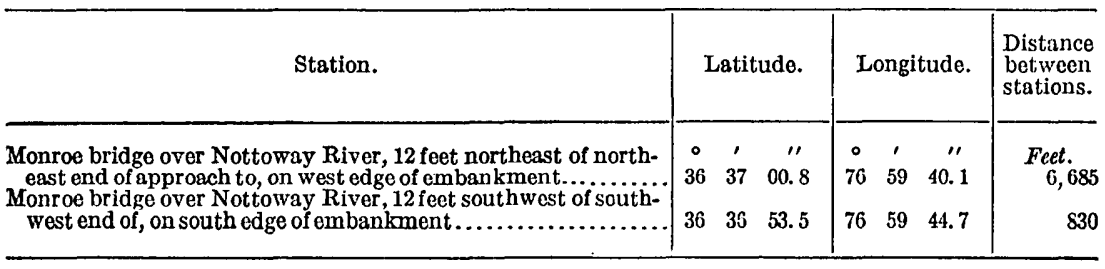

Magnetic declination through center of quadrangle, $4.2^{\circ} \mathrm{W} . ;$ in northwest part of quadrangle, $4.3^{\circ} \mathrm{W}$.

North along highways near east border of quadrangle.

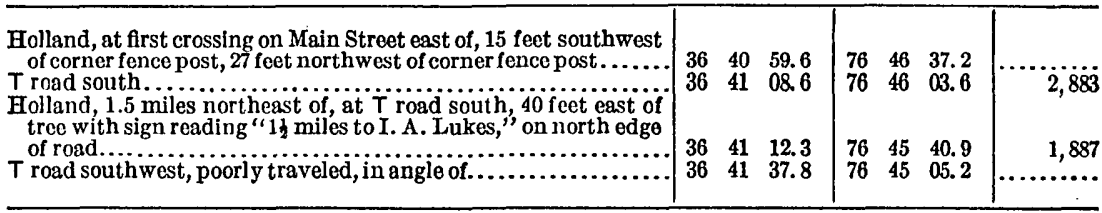

Magnetic declination for north half of east border of quadrangle, $5.5^{\circ} \mathrm{W}$.

EMPORIA QUADRANGLE.

Along Southern Ry. west from Emporia. (By J. C. Fales in 1918.)

\begin{tabular}{|c|c|c|c|c|c|c|c|}
\hline \multirow[t]{2}{*}{ Station. } & \multicolumn{3}{|c|}{ Latitude. } & \multicolumn{3}{|c|}{ Longitude. } & \multirow{2}{*}{$\begin{array}{r}\begin{array}{l}\text { Distance } \\
\text { betwcen } \\
\text { stations. }\end{array} \\
\text { Fect. }\end{array}$} \\
\hline & - & , & $\prime \prime$ & $\circ$ & , & $\prime \prime$ & \\
\hline Water tank, north rail opposite spout of (distance from P. T. S. 6) & 36 & 41 & 41.5 & 77 & 32 & 40.1 & \\
\hline Milepost 75, center line of track opposite ...................... & 36 & 42 & 18.8 & 77 & 33 & 38.6 & 6,070 \\
\hline 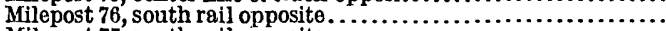 & 36 & 42 & 48. 6 & 77 & 34 & 33.1 & 5,365 \\
\hline Milepost 77 , south rail opposite..................... & 36 & 43 & 17.8 & 77 & 35 & 27.1 & 5,296 \\
\hline 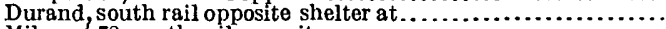 & 36 & 43 & 21.5 & 77 & 35 & 50.6 & 1,950 \\
\hline $\begin{array}{l}\text { Milepost } 78 \text {, south rail opposite. } \\
\text { Crossing of Emporia-Lawrencevilie road, } 90 \text { feet east of } \text { logging }\end{array}$ & 36 & 43 & 23.6 & 77 & 36 & 31.0 & 3,300 \\
\hline 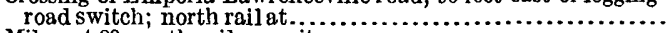 & 36 & 43 & 35.1 & 77 & 37 & 14.9 & 3,750 \\
\hline 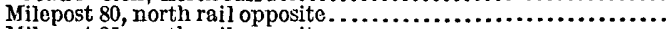 & 36 & 44 & 15.7 & 77 & 38 & 19.6 & 6,670 \\
\hline Milepost 85, north rail opposite............ & 36 & 44 & 56.7 & 77 & 42 & 52.2 & \\
\hline Racume depot, north rail opposite........... & 36 & 44 & 53.8 & 77 & 43 & 21.1 & 2,371 \\
\hline
\end{tabular}

South along highways near west border of quadrangle.

\begin{tabular}{|c|c|c|c|c|c|c|c|}
\hline 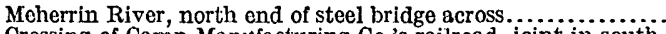 & & & 50.0 & & 44 & 57.5 & \\
\hline $\begin{array}{l}\text { Crossing of Camp Manufacturing Co.'s railroad, joint in south } \\
\text { rail at east edgo of road } \ldots \ldots \ldots \ldots \ldots \ldots \ldots \ldots \ldots \ldots \ldots \ldots\end{array}$ & 36 & 42 & 03.6 & 77 & 44 & 27.9 & \\
\hline 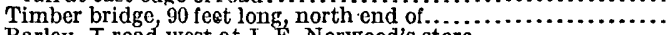 & 36 & 34 & 13.2 & 77 & 44 & 25.4 & \\
\hline 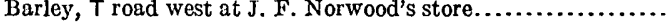 & 36 & 33 & 49.7 & 77 & 43 & 28.0 & \\
\hline
\end{tabular}

South along Atlantic Coast Line R. R. near east border of quadrangle. (By J. C. Fales In 1918.)

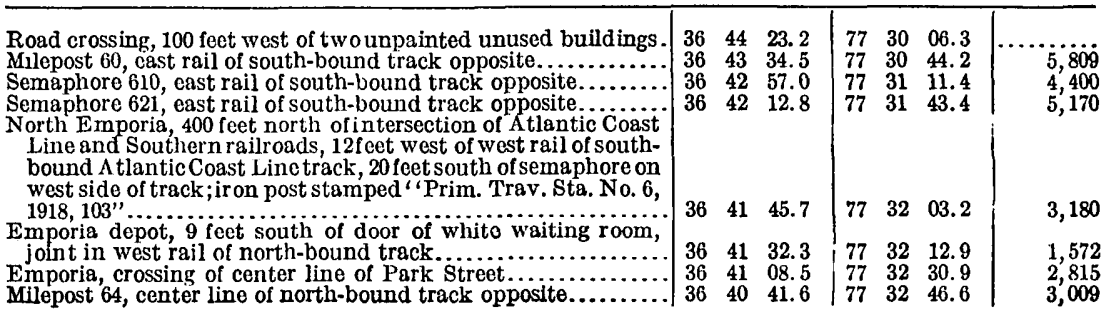


EMPORIA QUADRATGLE-Continued.

South along Atlantic Coast Line R. R. near east border of quadrangle-Continued.

\begin{tabular}{|c|c|c|c|c|c|c|}
\hline Station. & \multicolumn{2}{|c|}{ Latitude. } & \multicolumn{3}{|c|}{ Longitude. } & \multirow{2}{*}{$\begin{array}{c}\begin{array}{c}\text { Distance } \\
\text { between } \\
\text { stations. }\end{array} \\
\text { Feet. } \\
5,350 \\
5,345 \\
3,817 \\
6,770 \\
3,810 \\
6,807 \\
5,250 \\
4,944 \\
8,050\end{array}$} \\
\hline 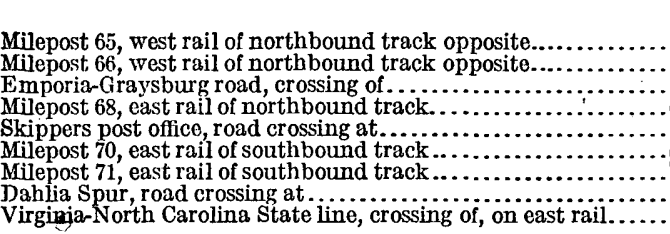 & $\begin{array}{cc}\circ & \text { ' } \\
36 & 39 \\
36 & 38 \\
36 & 38 \\
36 & 37 \\
36 & 36 \\
36 & 35 \\
36 & 34 \\
36 & 33 \\
36 & 32\end{array}$ & $\begin{array}{c}\prime \prime \\
50.4 \\
59.3 \\
22.2 \\
17.0 \\
42.5 \\
38.1 \\
46.4 \\
59.2 \\
42.6\end{array}$ & $\begin{array}{c}\circ \\
77 \\
77 \\
77 \\
77 \\
77 \\
77 \\
77 \\
77 \\
77\end{array}$ & $\begin{array}{l}\text { ' } \\
33 \\
33 \\
33 \\
33 \\
32 \\
32 \\
32 \\
32 \\
31\end{array}$ & $\begin{array}{c}\prime \prime \\
03.4 \\
19.9 \\
28.8 \\
10.0 \\
51.3 \\
27.1 \\
32.2 \\
16.5 \\
49.7\end{array}$ & \\
\hline
\end{tabular}

Magnetic declination for west border of quadrangle, $3^{\circ} 50^{\prime} \mathrm{W}$.

East along Southern Ry. from Emporia. (By H. J. Switzer in 1918.)

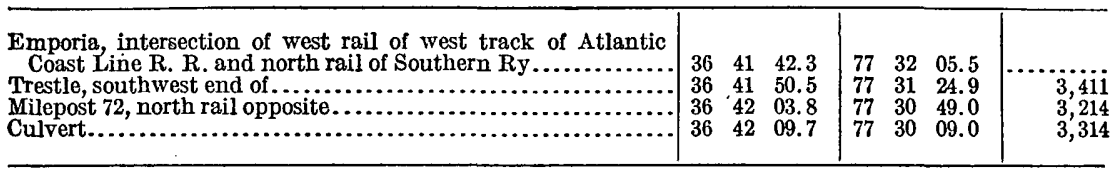

Southwest from Emporia along highways through center of quadrangle. (By J. C. Fales in 1918.)

\begin{tabular}{|c|c|c|c|c|c|c|c|}
\hline $\begin{array}{l}\text { Road, opposite sign reading " } 2 \text { miles to First National Bank". } \\
\text { Fork west... } \\
\text { Road, opposite sign reading "“4 miles to Lverett's department }\end{array}$ & $\begin{array}{l}36 \\
36\end{array}$ & $\begin{array}{l}40 \\
39\end{array}$ & $\begin{array}{l}11.0 \\
51.5\end{array}$ & $\begin{array}{l}77 \\
77\end{array}$ & $\begin{array}{l}33 \\
34\end{array}$ & $\begin{array}{l}33.7 \\
17.9\end{array}$ & 4,115 \\
\hline 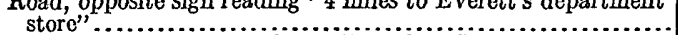 & 36 & 39 & 19.0 & 77 & 35 & 33.0 & 6,956 \\
\hline Railroad crossing on Hitchcock Branch of Southern $\mathbf{R y} . . . . .$. & 36 & 38 & 59.6 & 77 & 36 & 05.9 & 3,331 \\
\hline 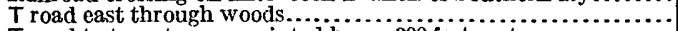 & 36 & 38 & 31.1 & 77 & 36 & 37.0 & 3,847 \\
\hline $\begin{array}{l}\text { T road to two-story unpainted house } 300 \text { feet east................ } \\
\text { Schoolhouse, new, light brown, } 150 \text { feet east of road, road }\end{array}$ & 36 & 37 & 48.4 & 77 & 37 & 27.4 & 5,973 \\
\hline 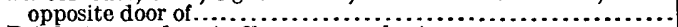 & 36 & 37 & 20.3 & 77 & 37 & 43.5 & 3,139 \\
\hline Brink store and post oflice, crossroads at.............. & 36 & 37 & 04.7 & 77 & 38 & 05.2 & 2,378 \\
\hline 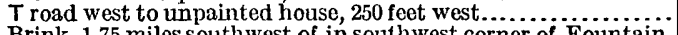 & 36 & 36 & 39.6 & 77 & 39 & 09.2 & 5,810 \\
\hline $\begin{array}{l}\text { Brink, } 1.75 \text { miles southwest of in southwest corner of Fountain } \\
\text { Creek Church yard, large unpainted building, } 100 \text { feet south of, } \\
20 \text { feet west of center line of road; iron post stamped "Prim. }\end{array}$ & & & & & & & \\
\hline Trav. Sta. No. 14, $1918^{\prime \prime} \ldots \ldots \ldots \ldots \ldots \ldots \ldots \ldots$ & 36 & 36 & 30.2 & 77 & 39 & 44.7 & 3,056 \\
\hline T road east past dismantled house 300 feet east of roa & 36 & 36 & 06.3 & 77 & 41 & 05.1 & 6,986 \\
\hline 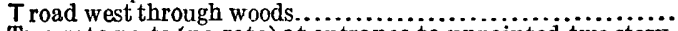 & 36 & 35 & 49.1 & 77 & 41 & 44.8 & 3,677 \\
\hline Two gate posts (no gate) at entrance to unpainted two-story & 36 & 35 & 22 & 77 & 42 & & \\
\hline 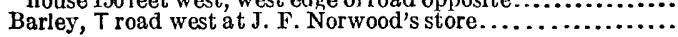 & 36 & 33 & 49.7 & 77 & 43 & $\begin{array}{l}12.0 \\
28.0\end{array}$ & $\begin{aligned} 3,210 \\
11,228\end{aligned}$ \\
\hline
\end{tabular}

East along highways from west border to center of quadrangle.

\begin{tabular}{|c|c|c|c|c|c|c|c|}
\hline $\begin{array}{l}\text { Troad north past unpainted negro house......................... } \\
\text { Wagon gate to two-story white house of Bert Mitcheli, road op- }\end{array}$ & 36 & & 58.3 & & & 41.9 & \\
\hline 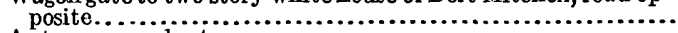 & 36 & 39 & 48.3 & 77 & 43 & 34.9 & 5,555 \\
\hline 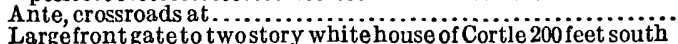 & 36 & 39 & 53.8 & 77 & 43 & 10.5 & 2,065 \\
\hline 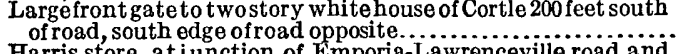 & 36 & 39 & 57.1 & 77 & & 19.9 & 4,136 \\
\hline $\begin{array}{l}\text { Harris store, at i unction of Emporia-Lawrenceville road and } \\
\text { road to Brink post office, atsouthwest corner of road T; iron } \\
\text { post stamped "Prim. Trav. Sta. No. } 26,1918 \text { "................ }\end{array}$ & 36 & 40 & 05.1 & 77 & 41 & 17.2 & 5,170 \\
\hline $\begin{array}{l}\text { Two gate posts } \\
\text { pointin road between } . . . \ldots \ldots \ldots \ldots \ldots \ldots \ldots \ldots \ldots \ldots \ldots \ldots \ldots \ldots \ldots \ldots\end{array}$ & 36 & 39 & 46. 3 & 77 & 41 & 09.8 & 2,003 \\
\hline Large wooden gateat entrancetofarm yard of J. H. Newson & 36 & 39 & 32.1 & 77 & 40 & 17. 1 & 4,523 \\
\hline 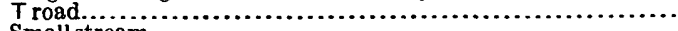 & 36 & 39 & 11.2 & 77 & 39 & 21.7 & 4,986 \\
\hline Small stream & 36 & 38 & 24.7 & 77 & 38 & 55.9 & 5,150 \\
\hline Timber bridge 100 feet by 16 feet, north end of, a t center line... & 36 & 37 & 59.4 & 77 & 38 & 44.3 & 2,732 \\
\hline iron post stamped "Prim. Trav. Sta. No. 27, 1918"......... & 36 & 37 & 04.9 & 77 & 38 & 04.7 & 6,387 \\
\hline
\end{tabular}

Magnetic declination for center of quadrangle, $3^{\circ} 40^{\circ} \mathrm{W}$.; for west part of quadrangle, $4^{\circ} 00^{\circ} \mathrm{W}$. 
EMPORIA QUADRATGLE-Continued.

Along rallroad and hlghways through center of quadrangle. (By H. J. Switzer in 1918.)

\begin{tabular}{|c|c|c|c|c|c|c|}
\hline Station. & \multicolumn{2}{|c|}{ Latitude. } & \multicolumn{3}{|c|}{ Longitudo. } & $\begin{array}{l}\text { Distance } \\
\text { between } \\
\text { stations. }\end{array}$ \\
\hline 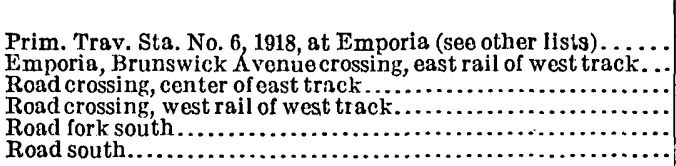 & $\begin{array}{cc}\circ & \text { ' } \\
36 & 41 \\
36 & 41 \\
36 & 40 \\
36 & 40 \\
36 & 39 \\
36 & 39\end{array}$ & $\begin{array}{l}\quad \prime \prime \\
45.70 \\
30.6 \\
55.7 \\
36.4 \\
48.9 \\
26.2\end{array}$ & $\begin{array}{c}\circ \\
77 \\
77 \\
77 \\
77 \\
77 \\
77\end{array}$ & $\begin{array}{l}\text { ' } \\
32 \\
32 \\
32 \\
32 \\
31 \\
31\end{array}$ & $\begin{array}{l}\prime \prime \\
03.20 \\
14.3 \\
39.6 \\
48.8 \\
56.2 \\
07.6\end{array}$ & \begin{tabular}{r} 
Feet. \\
\hdashline 1,777 \\
4,084 \\
2,094 \\
6,436 \\
4,578
\end{tabular} \\
\hline
\end{tabular}

HEATHSVILLE QUADRANGLE.

The following geographic positions in the Heathsville quadrangle were determined by E. L. McNair in 1916 and are based on the positions of the U. S. Coast and Geodetic Survey stations Hack and Great Wicomico Lighthouse.

South along highways through center of quadrangle.

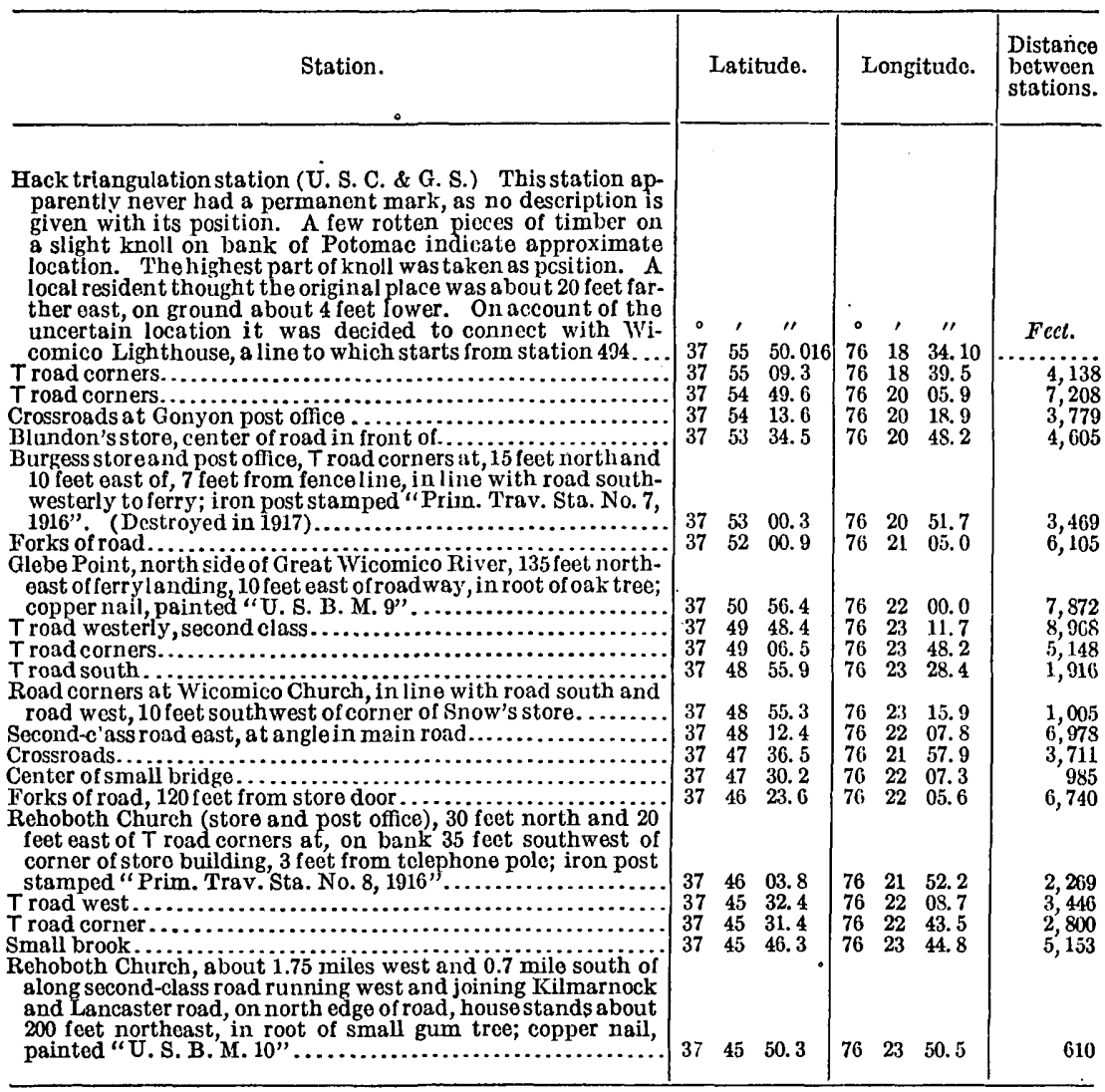


HEATHSVILIE QUADRANGLE-Continued.

Along highways near south border of quadrangle.

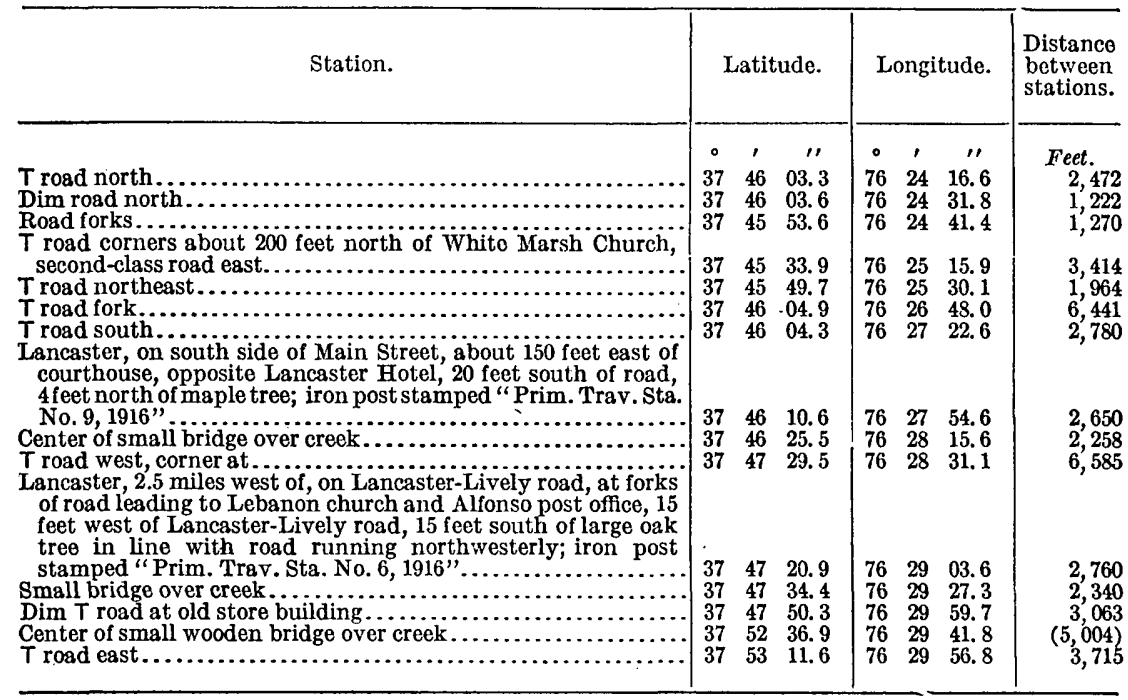

Along highways diagonally through center of quadrangle from northwest to southeast.

Road east to camp grounds. .

Troad corners near Heathsville new high school...............

Heathsville, on public square, 200 feet north of courthouse, 25

feet west of sidewalk; stone post with tablet in top marked

"U. S. Coast and Geodetic Survey magnetic station" .........

Second-class road southwest. .............................

T road north.

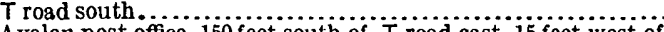

Avalon post office, 150 feet south of, T road east, 15 feet west of road, 15 feet east of wagon shop, under mulberry tree; iron post stamped "Prim. Trav. Sta. No. 12, 1916"..............

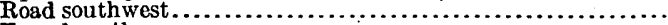

$T$ road south $\ldots \ldots \ldots \ldots \ldots \ldots \ldots \ldots \ldots \ldots \ldots \ldots \ldots \ldots \ldots \ldots \ldots \ldots$

$T$ road south $\ldots \ldots \ldots \ldots \ldots \ldots \ldots \ldots \ldots \ldots \ldots \ldots \ldots \ldots \ldots \ldots \ldots \ldots$

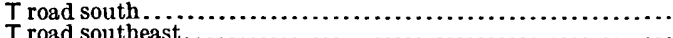

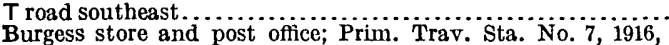

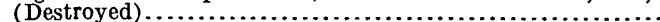

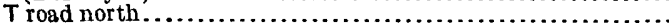

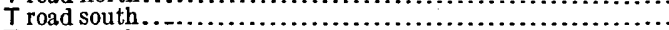

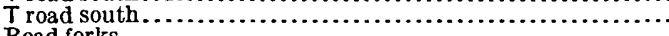

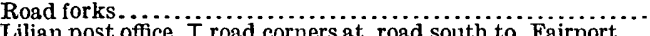

Beverlyville post office, $T$ road corners, in front of store........

Road forks, south road to Reedville, southeasterly road to Flee-

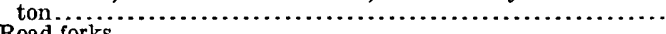

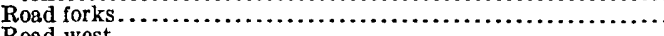

Great Wicomico Lighthouse (U. S. Coast \& Geodetic Survey).

\begin{tabular}{|c|c|c|c|c|c|c|}
\hline $\begin{array}{l}37 \\
37\end{array}$ & $\begin{array}{l}54 \\
55\end{array}$ & $\begin{array}{l}03.7 \\
20.1\end{array}$ & $\begin{array}{l}76 \\
76\end{array}$ & $\begin{array}{l}29 \\
29\end{array}$ & $\begin{array}{l}23.4 \\
04.7\end{array}$ & $\begin{array}{l}4,906 \\
7,874\end{array}$ \\
\hline $\begin{array}{l}37 \\
37 \\
37 \\
37\end{array}$ & $\begin{array}{l}\mathbf{5 5} \\
\mathbf{5 4} \\
\mathbf{5 5} \\
\mathbf{5 4}\end{array}$ & $\begin{array}{l}03.1 \\
56.4 \\
02.3 \\
57.3\end{array}$ & $\begin{array}{l}76 \\
76 \\
76 \\
76\end{array}$ & $\begin{array}{l}28 \\
27 \\
26 \\
26\end{array}$ & $\begin{array}{l}21.4 \\
25.3 \\
43.9 \\
21.9\end{array}$ & $\begin{array}{l}3,870 \\
4,552 \\
3,366 \\
1,838\end{array}$ \\
\hline $\begin{array}{l}37 \\
37 \\
37 \\
37 \\
37 \\
37\end{array}$ & $\begin{array}{l}54 \\
53 \\
53 \\
53 \\
53 \\
53\end{array}$ & $\begin{array}{l}24.4 \\
32.6 \\
22.2 \\
27.9 \\
21.6 \\
33.5\end{array}$ & $\begin{array}{l}76 \\
76 \\
76 \\
76 \\
76 \\
76\end{array}$ & $\begin{array}{l}25 \\
25 \\
24 \\
22 \\
22 \\
21\end{array}$ & $\begin{array}{l}28.2 \\
08.1 \\
18.4 \\
52.8 \\
29.7 \\
47.5\end{array}$ & $\begin{array}{l}5,443 \\
5,479 \\
4,120 \\
6,886 \\
1,954 \\
3,593\end{array}$ \\
\hline $\begin{array}{l}37 \\
37 \\
37 \\
37 \\
37 \\
37 \\
37\end{array}$ & $\begin{array}{l}53 \\
52 \\
52 \\
52 \\
52 \\
51 \\
51\end{array}$ & $\begin{array}{l}00.0 \\
52.2 \\
51.1 \\
06.9 \\
01.8 \\
41.4 \\
54.0\end{array}$ & $\begin{array}{l}76 \\
76 \\
76 \\
76 \\
76 \\
76 \\
76\end{array}$ & $\begin{array}{l}20 \\
20 \\
20 \\
18 \\
18 \\
18 \\
16\end{array}$ & $\begin{array}{l}51.7 \\
08.1 \\
01.5 \\
38.0 \\
17.3 \\
03.1 \\
56.0\end{array}$ & $\begin{array}{r}\mathbf{5}, \mathbf{5 9 1} \\
\mathbf{3}, \mathbf{6 0 0} \\
\mathbf{5 3 5} \\
\mathbf{8}, 060 \\
\mathbf{1}, \mathbf{7 4 0} \\
\mathbf{2}, \mathbf{3 5 6} \\
\mathbf{5}, 528\end{array}$ \\
\hline $\begin{array}{l}37 \\
37 \\
37 \\
37\end{array}$ & $\begin{array}{l}51 \\
50 \\
49 \\
48\end{array}$ & $\begin{array}{l}00.5 \\
17.5 \\
49.5 \\
15.38\end{array}$ & $\begin{array}{l}76 \\
76 \\
76 \\
76\end{array}$ & $\begin{array}{l}16 \\
15 \\
16 \\
16\end{array}$ & $\begin{array}{l}22.8 \\
46.4 \\
03.5 \\
04.32\end{array}$ & $\begin{array}{l}6,026 \\
5,249 \\
3,146 \\
9,525\end{array}$ \\
\hline
\end{tabular}

Magnetic declination for centcr of quadrangle, north and south, $5^{\circ} 43^{\prime} \mathrm{W}$.; for south border of quadrangle $5^{\circ} 46^{\prime} \mathrm{W}$.; for west border of quadrangle, $5^{\circ} 38^{\prime} \mathrm{W}$.; through quadrangle, northwest to southeast, $5^{\circ} 51^{\prime} \mathrm{W}$.

Along highways in the southwest corner of quadrangle. (Checked spur line.)

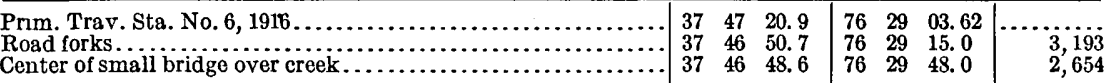

Along highways near west border of quadrangle.

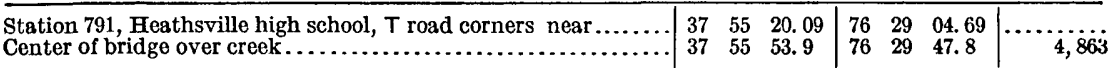

Magnetic declination near west border of quadrangle; $5^{\circ} 57^{\prime} \mathrm{W}$.; in southwest corner of quadrangle, $5^{\circ} 40^{\circ} \mathrm{W}$. 
HOMEVILLE QUADRANGLE.

West along Norfolk \& Western Ry. and highways near north border of quadrangle. (By J. H. Wilson in 1916-17.)

\begin{tabular}{|c|c|c|c|c|c|c|}
\hline Station. & I,atit & ide. & Lo & ngit & ude. & $\begin{array}{l}\text { Distance } \\
\text { between } \\
\text { stations. }\end{array}$ \\
\hline 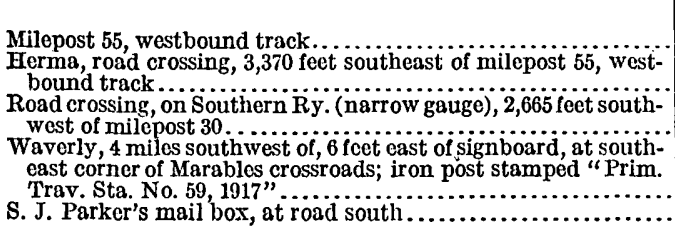 & $\begin{array}{ll}\circ & , \\
36 & 59 \\
36 & 59 \\
36 & 59\end{array}$ & $\begin{array}{l}\prime \prime \\
56.0 \\
35.0 \\
45.9\end{array}$ & $\begin{array}{l}0 \\
77 \\
77 \\
77\end{array}$ & $\begin{array}{l}\prime \\
02 \\
01 \\
08\end{array}$ & $\begin{array}{l}\prime \prime \\
13.6 \\
41.4 \\
11.8\end{array}$ & $\begin{array}{r}\text { Feet. } \\
\quad 3,370 \\
\ldots \ldots \ldots\end{array}$ \\
\hline
\end{tabular}

Magnetic declination near north border of quadrangle, $4^{\circ} 40^{\prime} \mathrm{W}$.

From Marlboro crossroads southwest along highway and Southern Ry. (narrow gage) to Lumberton. (By A. C. Schilling in 1918.)

T road west.

Gray's lumber railroad, a narrow gage spur of Southern $\mathrm{Ry}$., crossing on.

County-road crossing

Railroad switch east.

Road crossing, 110 feet south of point of switch

point of switch. ............

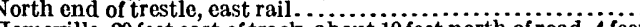
Homeville, 20 feet east of track, about 10 feet north of road, 4 feet
west of crossing sign; iron post stamped "Prim. Trav. Sta. No. $1 "$.

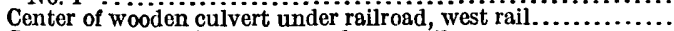

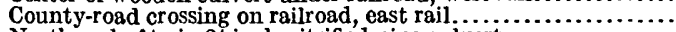

North end of twin 24 -inch vitrified pipe culvert..............

Road crossing.

Burt's switch, 714 feet northeast of, at north end of wooden trestlo under railroad.

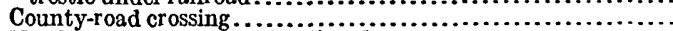

North end of trestle under railioad

Nottoway River, north end of large wooden trestle over; west

Nottoway River, south end of large bridge over, west rail, 270 feet northeast of county-road crossing leading to sawmill...... North end of trestlo over small creek.

Lumberton, 20 feet west of track, about 10 feet north of road; iron post stamped "Prim. Trav. Sta. No. 2"

\begin{tabular}{|lll|lll|r}
36 & 59 & 42.9 & 77 & 08 & 40.7 & $\ldots \ldots \ldots$ \\
36 & 59 & 12.3 & 77 & 08 & 45.9 & 3,118 \\
36 & 58 & 24.8 & 77 & 09 & 05.0 & 5,051 \\
36 & 58 & 17.5 & 77 & 09 & 06.6 & 747 \\
36 & 57 & 52.6 & 77 & 09 & 25.5 & 2,949 \\
36 & 57 & 23.9 & 77 & 09 & 30.2 & 2,928 \\
& & & & & & \\
36 & 56 & 55.3 & 77 & 10 & 19.04 & 4,905 \\
36 & 56 & 37.7 & 77 & 10 & 32.5 & 2,089 \\
36 & 56 & 10.4 & 77 & 10 & 40.7 & 2,841 \\
36 & 55 & 56.2 & 77 & 10 & 51.4 & 1,676 \\
36 & 55 & 23.2 & 77 & 11 & 02.9 & 3,464 \\
& & & & & & \\
36 & 54 & 45.1 & 77 & 11 & 08.95 & 3,888 \\
36 & 54 & 20.0 & 77 & 11 & 23.4 & 2,794 \\
36 & 53 & 53.3 & 77 & 11 & 40.3 & 3,032 \\
& & & & & & \\
36 & 53 & 42.4 & 77 & 11 & 44.6 & 1,153 \\
& & & & & & \\
36 & 53 & 31.8 & 77 & 11 & 51.4 & 1,209 \\
36 & 53 & 00.9 & 77 & 12 & 15.8 & 3,695 \\
36 & 52 & 53.7 & 77 & 12 & 39.1 & 2,028 \\
& & & & & & \\
& & & & & &
\end{tabular}

118

747

2,949

2,928

4,905

,841

, 676

888

, 794

, 032

1,153

, 209

, 028

East along highways through center of quadrangle.

North end of bridge over creek.

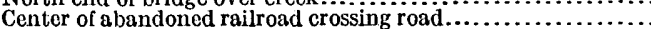

T road to Littleton.

Troad west.

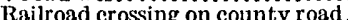

West end of ir on bridge.

Troad south, farm road, 730 feet southwest of another farm road.

Troad south, farm road.

Littleton, 80 feet north of northwest corner of post office and general store, 119 feet south of road forks, 16 fect east of center of road to Yale, 37 feet west of center of road to Sebrell; iron post stamped "Prim. Trav. Sta. No. 3"

$T$ road east

Surry Lumber Co.'s railroad right of way, 21 feet north of, 18 feet east of center of county road; iron post stamped "Prim.

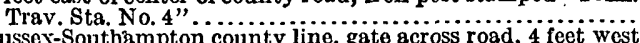
Sussex-Southampton county line, gate across road, 4 feet wes

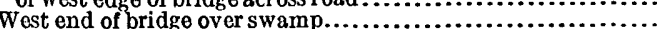

West end of bridge over swamp.

Center of gate across r oad.

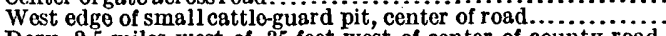
Dory, 2.5 miles west of, 35 fect west of center of county road between Waverly and Sebrell, 12 feet south of Surry Lumber Co's, right of way, in forks; iron post stamped " Prim. Trav.

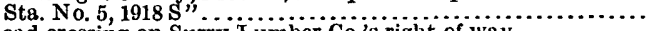

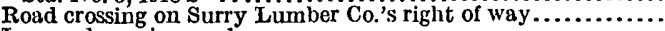
Log road crossing road.

Dory, beginning of spur track east to

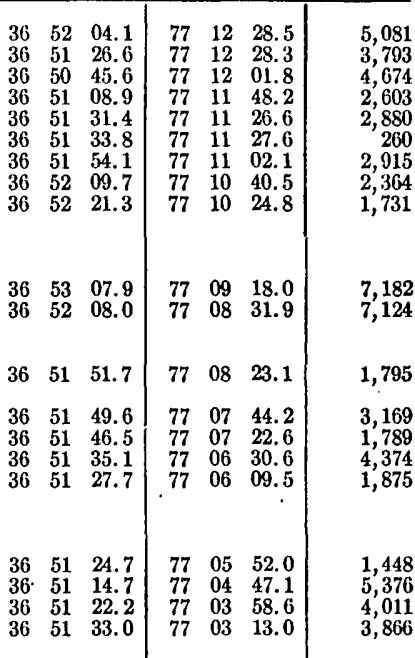


HOMEVILLE QUADRANGLE-Continued.

From Dory northeast along Surry, Sussex \& Southampton Ry. (narrow gage) to east border of quadrangle.

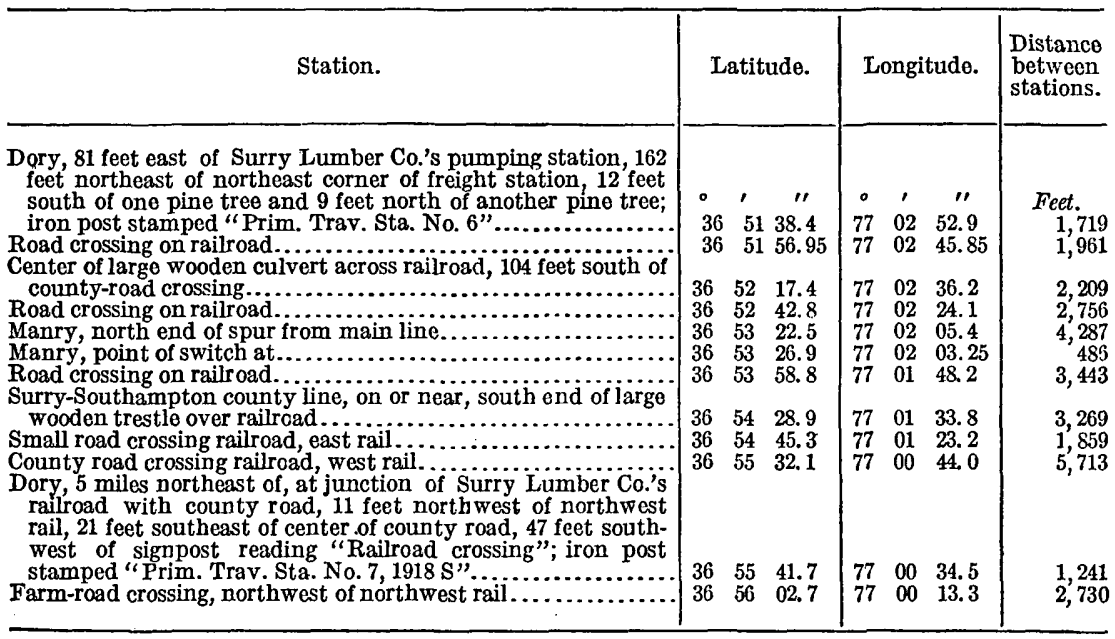

Along Norfolk \& Western Ry. in northeast part of quadrangle.

Road crossing, east rail

Wakefield, 1.5 miles northwest of, southwest side of track, in north face of concrete foundation to semaphore; bronze tablet set vertically, stamped " 1111917 "

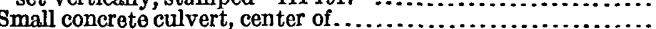

Herma, road crossing, 3,370 feet southeast of milepost 55 , north-

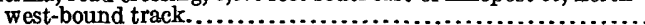

\begin{tabular}{|lll|lll|r}
36 & 58 & 38.2 & 77 & 00 & 14.4 & $\ldots \ldots \ldots$. \\
36 & 58 & 53.3 & 77 & 00 & 37.5 & \\
36 & 59 & 08.1 & 77 & 01 & 00.0 & 2,421 \\
& & & & \\
36 & 59 & 35.0 & 77 & 01 & 41.4 & 4,321 \\
\hline
\end{tabular}

Southeast along Surry, Sussex \& Southampton Ry. (narrow gage). (By A. C. Schilling in 1918.)

Dory, Prim. Trav. Sta. No. 6 (previously described)............

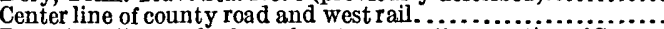

Dory, 1.5 miles south of, nor theast corner of intersection of Surry, Sussex \& Southampton Ry. and road to Sebrell, 6 feet cast of east rail, 22 feet south of intersection of county road and east rail, 27 feet southeast of signpost; iron post stamped "Prim. Trav. Sta. No. 9"'

Center of large wcoden culveit

Center line of county road between Courtland and Ivor..........

Center line of iron culvert, 1,050 feet northwest of center of wooden culvert........................................

Vicksville, north side of county road between Courtland and Ivor, 95 feet west of west rail of Surry, Sussex \& Scuthampton Ry., No. 10".........................................

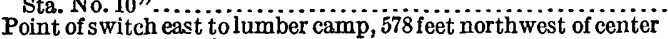

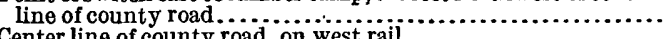

Center line of county road, on west rail ................................

south rail of Virginian Ry. 8 feet west of west rail of Surry, Sussex \& Southampton Ry.; iron post stamped "Prim. Trav. Sta. No. 11" (in Ivor quadrangle).

\begin{tabular}{|rrr|rrr|r}
36 & 51 & 38.4 & 77 & 02 & 52.9 & $\ldots \ldots \ldots$, \\
36 & 51 & 12.7 & 77 & 03 & 06.7 & 2,832 \\
& & & & & & \\
& & & & & & \\
36 & 51 & 04.7 & 77 & 03 & 07.6 & \\
36 & 50 & 14.1 & 77 & 02 & 24.7 & 813 \\
36 & 50 & 00.0 & 77 & 02 & 12.6 & 6,193 \\
36 & 49 & 07.4 & 77 & 01 & 27.5 & 1,738 \\
& & & & & & 6,454 \\
36 & 48 & 50.6 & 77 & 01 & 14.5 & 2,004 \\
36 & 48 & 39.6 & 77 & 01 & 03.6 & 1,428 \\
36 & 47 & 29.4 & 77 & 00 & 03.2 & 8,629 \\
& & & & & & \\
36 & 46 & 29.0 & 76 & 59 & 17.3 & 7,165
\end{tabular}

West along Virginian Ry.

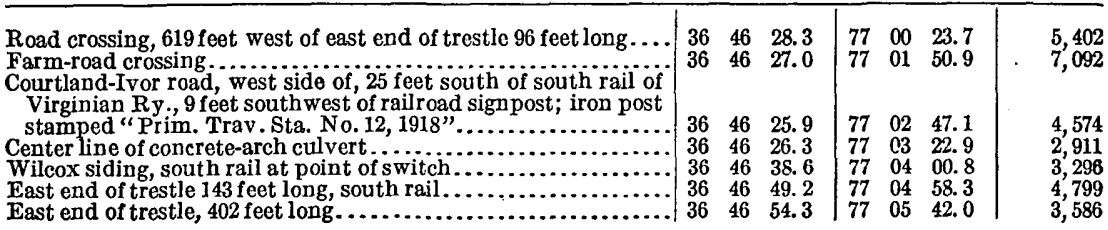


HOMEVILIE QUADRANGLE-Continued.

West along Virginian Ry.-Continued.

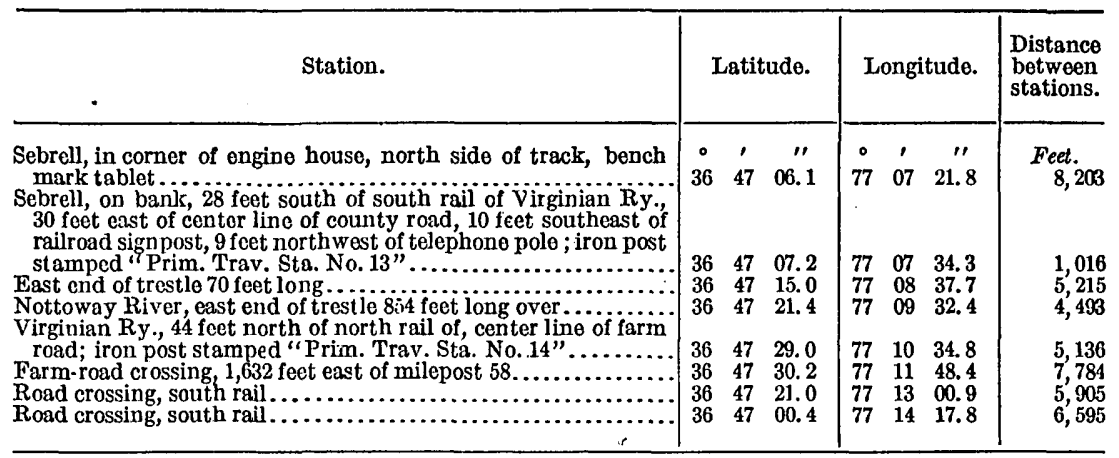

South along county road.

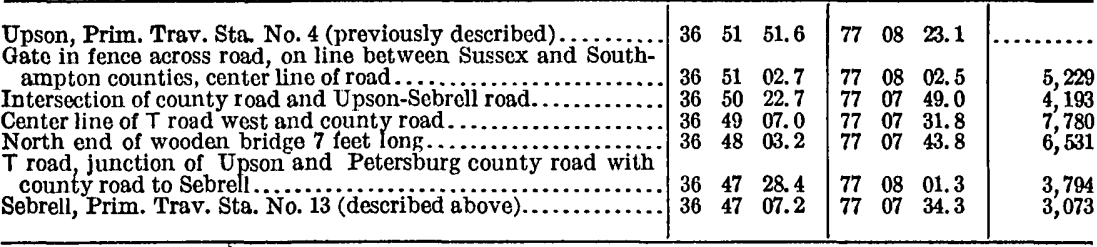

Along Southern Ry. south of Lumberton.

\begin{tabular}{|c|c|c|c|c|c|c|c|}
\hline 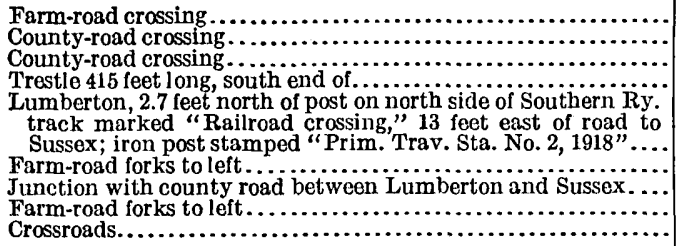 & $\begin{array}{l}36 \\
36 \\
36 \\
36 \\
36\end{array}$ & $\begin{array}{l}\mathbf{5 2} \\
53 \\
53 \\
53 \\
54\end{array}$ & $\begin{array}{l}53.6 \\
15.9 \\
42.4 \\
46.4 \\
17.8\end{array}$ & $\begin{array}{l}77 \\
77 \\
77 \\
77 \\
77\end{array}$ & $\begin{array}{l}12 \\
12 \\
13 \\
13 \\
14\end{array}$ & $\begin{array}{l}39.1 \\
50.7 \\
00.1 \\
46.2 \\
36.5\end{array}$ & $\begin{array}{l}3,480 \\
2,443 \\
2,787 \\
3,762 \\
5,182\end{array}$ \\
\hline
\end{tabular}

Magnetic declination for south part of quadrangle, $4.2^{\circ} \mathrm{W}$.

Southwest along highways in southwest corner of quadrangle. (By J. J. Phelan in 1917.)

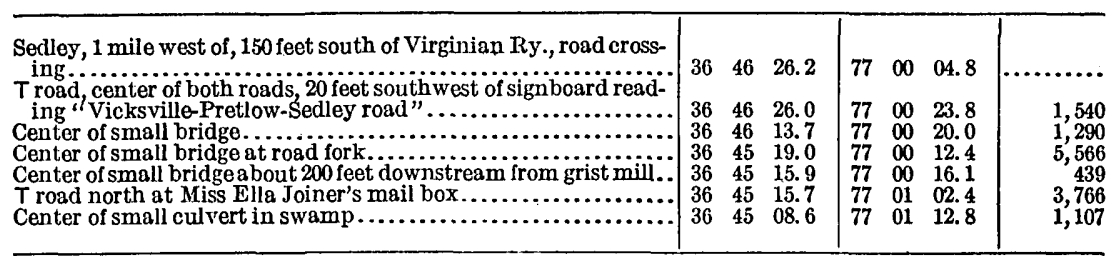

Magnetic declination along southwest edge of quadrangle, $4.2^{\circ} \mathrm{W}$. 


\section{IVOR QUADRANGIE.}

North along Surry, Sussex \& Southampton Ry. and highways near west border of quadrangle. (By A. C. Schllling in 1918.)

\begin{tabular}{|c|c|c|c|c|c|c|c|}
\hline Station. & \multicolumn{3}{|c|}{ Latitude. } & \multicolumn{3}{|c|}{ Longitude. } & $\begin{array}{l}\text { Distance } \\
\text { Detween } \\
\text { stations. }\end{array}$ \\
\hline 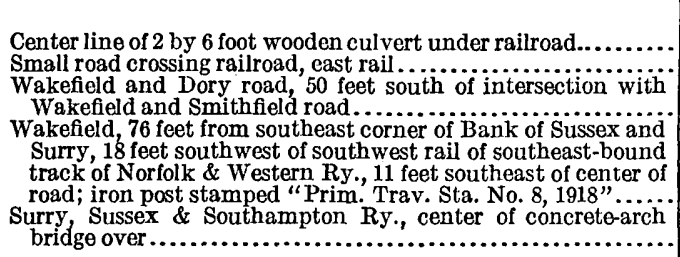 & $\begin{array}{r}\circ \\
36 \\
36 \\
36\end{array}$ & $\begin{array}{c}\prime \\
57 \\
57 \\
58\end{array}$ & $\begin{array}{c}\prime \prime \\
13.1 \\
33.6 \\
01.0\end{array}$ & $\begin{array}{c}\circ \\
76 \\
76 \\
76\end{array}$ & \begin{tabular}{c}
\multicolumn{1}{c}{} \\
59 \\
59 \\
59
\end{tabular} & $\begin{array}{c}\prime \prime \\
36.8 \\
35.9 \\
33.1\end{array}$ & $\begin{array}{r}\text { Feet. } \\
\cdots \cdots \\
2,0 ; 3 \\
2,785\end{array}$ \\
\hline
\end{tabular}

West along Virginian Ry. (By J. J. Charters in 1918.)

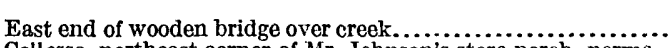
Collosse, northeast corner of Mr. Johnson's store porch, permanent bench mark marked "U. S. G. S." ....................

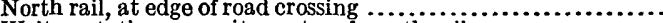

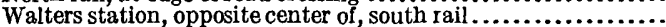

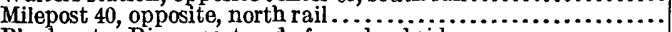

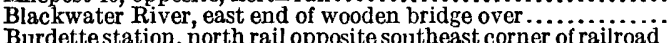
Burdette station, north rail opposite southeast corner of railroad

station

North rail opposite milepost 42 .

North rail opposite milepost $43 . \ldots \ldots \ldots \ldots \ldots \ldots \ldots \ldots \ldots \ldots \ldots . . . \ldots \ldots$

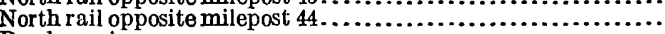

Road crossing.

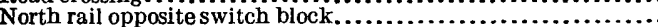

Sedley, southwest corner of railroad junction, 30 feet south of south rail of Virginian $\mathrm{Ry}$., 8 feet west of west rail of Surry, Sussex \& Southampton Ry.; iron post stamped "Prim. Trav. Sta. No. $11 "$

\begin{tabular}{|lll|lll|r}
36 & 45 & 24.4 & 76 & 48 & 41.3 & $\ldots \ldots \ldots \ldots$ \\
36 & 45 & 40.0 & 76 & 49 & 19.0 & \\
36 & 46 & 12.4 & 76 & 50 & 31.7 & 3,458 \\
36 & 46 & 18.3 & 76 & 51 & 03.6 & 6,767 \\
36 & 46 & 11.5 & 76 & 52 & 11.1 & 2,669 \\
36 & 46 & 05.2 & 76 & 53 & 08.4 & 5,533 \\
& & & & & & 4,707 \\
36 & 46 & 04.6 & 76 & 53 & 38.2 & 2,428 \\
36 & 46 & 09.1 & 76 & 54 & 19.8 & 3,414 \\
36 & 46 & 16.0 & 76 & 55 & 24.1 & 5,280 \\
36 & 46 & 22.9 & 76 & 56 & 28.4 & 5,280 \\
36 & 46 & 27.3 & 76 & 57 & 09.6 & 3,390 \\
36 & 46 & 30.3 & 76 & 58 & 15.1 & 5,340 \\
& & & & & & \\
& & & & & & \\
36 & 46 & 29.0 & 76 & 59 & 17.3 & 5,062 \\
& & & & & &
\end{tabular}

Northwest along Norfolk \& Western Ry. (By J. J. Charters in 1918.)

\begin{tabular}{|c|c|c|c|c|c|c|c|}
\hline \multirow{2}{*}{ 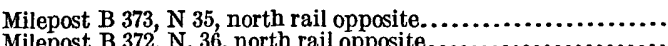 } & 36 & 48 & 57.4 & 76 & 45 & 26. & \\
\hline & 36 & 49 & & 76 & 46 & 16. & \\
\hline 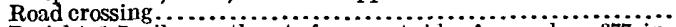 & 36 & 49 & 52.3 & 76 & & 49.7 & 3,52 \\
\hline $\begin{array}{l}\text { Dwight, } 1.7 \text { miles southeast of, on west side of semaphore } 377 \text {, in } \\
\text { top of foundation of iron ladder; bronze tablet stamped " } 71\end{array}$ & & & & & & & \\
\hline 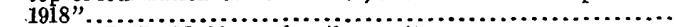 & 36 & 50 & 23.0 & 76 & 47 & 36.5 & 4,91 \\
\hline Milepost B 370, N. 38 , north rail opposite................ & 36 & 50 & 36.6 & 76 & 47 & 57. & 7 \\
\hline Milepost $\mathbf{B} 369$, N. 39 , north rail opposite.......... & 36 & 51 & 08.6 & 76 & 48 & 46.0 & 11 \\
\hline 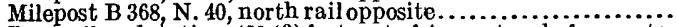 & 36 & 51 & 42.8 & 76 & 49 & 38.0 & 46 \\
\hline Zuni railroad station 450 (?) feet east of, in west end of concrete & & & & & & & \\
\hline 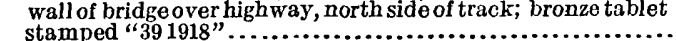 & 36 & 51 & 59.1 & 76 & 50 & 02.7 & 2,60 \\
\hline Blackwater River bridge. & 36 & 52 & 04.9 & 76 & 50 & 12.2 & \\
\hline Milepost $\mathrm{N} 42$, north rail opposite.... & 36 & 52 & 52.3 & 76 & 51 & 24.6 & 7,58 \\
\hline 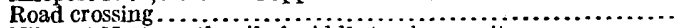 & 36 & 53 & 39. 7 & 76 & 52 & 36.9 & \\
\hline ail of middle track opposite........ & 36 & 53 & 54.5 & 76 & 52 & 59.8 & \\
\hline Ivor station, opposite center of, north rail of middle track & 36 & 54 & 24.0 & 76 & 53 & 45.0 & 13 \\
\hline Milepost B $362, \mathrm{~N} 46$, north rail opposite........ & 36 & 55 & 00.1 & 76 & 54 & 40.2 & \\
\hline 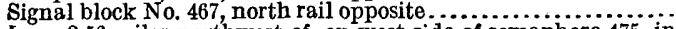 & 36 & 55 & 21.5 & 76 & 55 & 13.0 & \\
\hline Ivor, 2.56 miles northwest of, on west side of semaphore 475, in & & & & & & & \\
\hline “Elev, $79,1918 " \ldots \ldots \ldots$ & 36 & 55 & 47.4 & 76 & 55 & 52. & 4,141 \\
\hline 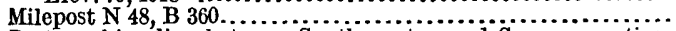 & 36 & 56 & 05.8 & 76 & 56 & 20.8 & 963 \\
\hline Post marking line between Southampton and Sussex counties, & & & & & & & \\
\hline $\begin{array}{l}300 \text { feet northwest of milepost } N 49 \text {, B } 359 \text {; north rail of north } \\
\text { track opposite. }\end{array}$ & & & & & & & \\
\hline 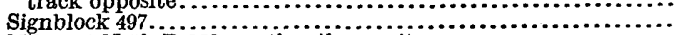 & $\begin{array}{l}36 \\
36\end{array}$ & $\begin{array}{l}56 \\
56\end{array}$ & $\begin{array}{l}40.2 \\
59.2\end{array}$ & 76 & 57 & 42. & 3,05 \\
\hline 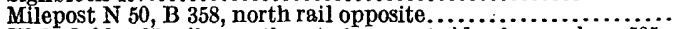 & 36 & 57 & 11.4 & 76 & 58 & 01.4 & 1,95 \\
\hline Wakefield, 1.07 miles southeast of, on west side of semaphore 505 , & & & & & & & \\
\hline 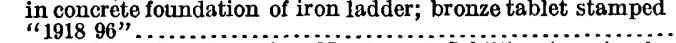 & 36 & 57 & & 76 & 58 & & \\
\hline Wakefield, Prim. Trav. Sta. No. 8,1918 , Schilling (previo & & & & & & 34.4 & \\
\hline described)..... & 36 & 58 & 04.5 & 76 & 59 & 23.4 & 5,100 \\
\hline
\end{tabular}


IVOR QUADRANGLE-Continued.

South along highways near center of quadrangle.

\begin{tabular}{|c|c|c|c|c|c|c|c|}
\hline \multirow[t]{2}{*}{ Station. } & \multicolumn{3}{|c|}{ Latitude. } & \multicolumn{3}{|c|}{ Longitude. } & \multirow{3}{*}{$\begin{array}{c}\begin{array}{c}\text { Distance } \\
\text { between } \\
\text { stations. }\end{array} \\
\text { Fet. }\end{array}$} \\
\hline & 0 & ' & $\prime \prime$ & & ' & & \\
\hline 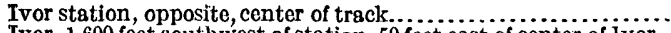 & 36 & 54 & 24.0 & 76 & 53 & 45.0 & \\
\hline $\begin{array}{l}\text { Ivor, } 1,600 \text { feet southwest of station, } 50 \text { feet east of center of Ivor- } \\
\text { Burdette road, } 91 \text {.1 feet from east corner of brick foundation } \\
\text { of residence, } 100.8 \text { feet from northeast corner of church founda- }\end{array}$ & & 54 & & & & & \\
\hline $\begin{array}{l}\text { tion; in northeast corner of church lot; iron post stamped }(?) \ldots \\
\text { Junction of Courtland road } . \ldots \ldots \ldots \ldots \ldots \ldots \ldots \ldots \ldots \ldots \ldots \ldots\end{array}$ & $\begin{array}{l}36 \\
36\end{array}$ & $\begin{array}{l}54 \\
53\end{array}$ & $\begin{array}{l}13.9 \\
55.5\end{array}$ & $\begin{array}{l}76 \\
76\end{array}$ & $\begin{array}{l}54 \\
54\end{array}$ & $\begin{array}{l}00.2 \\
45.7\end{array}$ & $\begin{array}{l}1,607 \\
4,134\end{array}$ \\
\hline Center line of road opposite milepost $2 . . . \ldots \ldots \ldots . . . . . . . . . . .$. & 36 & 53 & 05.5 & 76 & 54 & 38.2 & 5,097 \\
\hline Edge of drive southwest through woods. & 36 & 52 & 39.0 & 76 & 54 & 35.4 & 2,693 \\
\hline 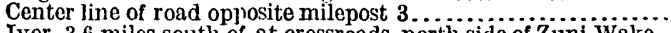 & 36 & 52 & 14.1 & & 54 & & 2,578 \\
\hline $\begin{array}{l}\text { Ivor, } 3.6 \text { miles south of, at crossroads, north side of Zuni-Wake- } \\
\text { field road and } 40 \text { fcet east of Ivor-Burdette road, } 49 \text { feet north- }\end{array}$ & 36 & 51 & 36.6 & 76 & 54 & 16.7 & 3.900 \\
\hline Third class $T$ road bearing north..................... & 36 & 50 & 58.4 & 76 & 53 & 52.5 & 4,343 \\
\hline 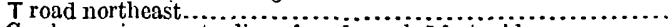 & 36 & 50 & 32.0 & 76 & 53 & 14.9 & 4,057 \\
\hline Creek crossing, center line of road, creek 5 feet wide. & 36 & 50 & 05.0 & 76 & 52 & 44.8 & 3,655 \\
\hline Junction of road from Zuni to Unity................... & 36 & 49 & 43.8 & 70 & 52 & 28.1 & 2,536 \\
\hline Small wooden bridge, at east end of, center line of road......... & 36 & 49 & 18.8 & 76 & 52 & 55.0 & 3,342 \\
\hline $\begin{array}{l}\text { Unity, in west face of northwest brick pier of foundation of Mr. } \\
\text { Stevenson's store, } 60 \text { feet east of T road; iron post stamped " } 49\end{array}$ & & & & & & & \\
\hline 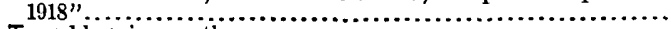 & 36 & 48 & 45.8 & 76 & 53 & 22.2 & 4,006 \\
\hline T road bcaring south...... & 36 & 48 & 22.5 & 76 & 53 & 03.8 & 2,792 \\
\hline T road bearing south . . . . . . . . . . . . . . . . . . . & 36 & 48 & 14.3 & 76 & 52 & 28.6 & 2,980 \\
\hline Center line of road opposite mail box 46 (Wilson) ........... & 36 & 47 & 56.1 & 76 & 52 & 45.8 & 2,314 \\
\hline Center line of road opposite mail box 56 N........ & 36 & 47 & 52.6 & 76 & 53 & 10.5 & 2,044 \\
\hline 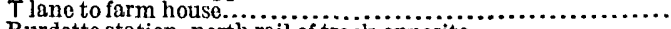 & 36 & 46 & 53.0 & 76 & 53 & 27.4 & 6,187 \\
\hline 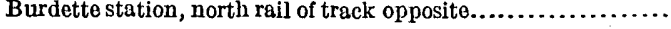 & 36 & 46 & 04.6 & 76 & 53 & 38.2 & 4,968 \\
\hline
\end{tabular}

North along Sussex Lumber Co.'s rallroad.

Ivor station, opposite, center of track

Crossing of road through woods.

Second-class road crossing

Crossing of Ivor and Smithfield road...

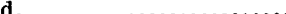

Wooden trestle, south end of .............................

Ivor, 3.1 miles northeast of, at road crossing, 10 feet south of road, 10 feet east of east rail, in top of small concrete culvert; bronze tablet stamped " 761918 "...

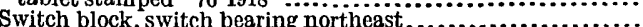

Second-class road bearing west, crossing of....................

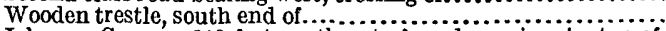

Johnson Corners, 116 feet southeast of road crossing, in top of concrete culvert; bronze tablet stamped " 401918 ".............

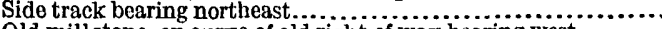

Old mill stone, on curve of old right of way bearing west.........

\begin{tabular}{|lll|lll|l}
36 & 54 & 24.0 & 76 & 53 & 45.0 & $\ldots \ldots \ldots$, \\
36 & 55 & 00.5 & 76 & 53 & 41.8 & 3,700 \\
36 & 55 & 30.5 & 76 & 53 & 26.1 & 3,291 \\
36 & 56 & 13.9 & 76 & 53 & 03.4 & 4,762 \\
36 & 56 & 37.4 & 76 & 52 & 51.2 & 2,584 \\
& & & & & & \\
36 & 56 & 53.3 & 76 & 52 & 42.9 & \\
36 & 57 & 46.4 & 76 & 52 & 27.4 & 1,742 \\
36 & 57 & 59.8 & 76 & 52 & 25.6 & 5,523 \\
36 & 58 & 17.7 & 76 & 51 & 56.7 & 1,363 \\
& & & & & & 2,967 \\
36 & 58 & 32.0 & 76 & 51 & 36.4 & 2,197 \\
36 & 59 & 09.3 & 76 & 51 & 15.8 & 4,124 \\
36 & 59 & 50.3 & 76 & 51 & 14.0 & 4,155 \\
& & & & & \\
\hline
\end{tabular}

Northeast along highway from Ivor to Bobs.

Ivor station, opposite, center of track

One-mile post of Sussex and Surrey road, center line of road

opposite................................................

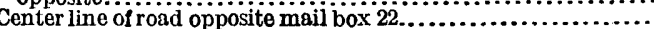

Lane bearing north.......................................

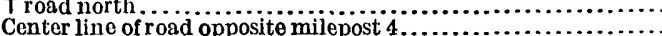

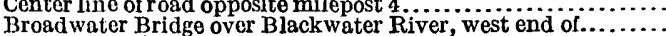

Broadwater Bridge over Blackwater River, west end of..........

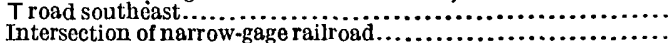

Milepost 6, center line of road opposite

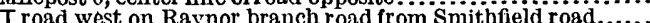

Sussex Lumber Co.'sproperty, centerline of road, opposite gate,

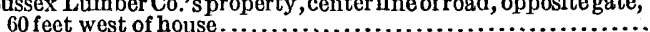

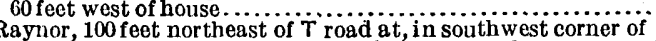

white church property, 12 feet south west of south west corner of church, 75 feet northeast of northeast corner of frame house;

Iron post stamped "Prim. Trav. Sta. No. 12, 1918 C".........

$T$ road east to Smithfield . ..............................

Bridge over creek, center line of road at south end of...............

T road west to Bobs..........................................

Drive to farm house .....................................

Wooden bridge over creek, south end of .....................

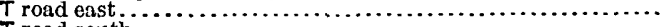

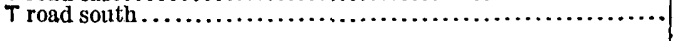

\begin{tabular}{|c|c|c|c|c|c|c|}
\hline 36 & 54 & 24.0 & & 53 & 45.0 & \\
\hline $\begin{array}{l}30 \\
36\end{array}$ & $\begin{array}{l}54 \\
54 \\
54 \\
54 \\
54 \\
54 \\
54 \\
54 \\
55 \\
55\end{array}$ & $\begin{array}{l}28.0 \\
25.0 \\
31.0 \\
37.8 \\
27.7 \\
19.7 \\
41.0 \\
54.3 \\
05.3 \\
33.1\end{array}$ & $\begin{array}{l}76 \\
76 \\
76 \\
76 \\
76 \\
76 \\
76 \\
76 \\
76 \\
76\end{array}$ & $\begin{array}{l}52 \\
51 \\
51 \\
50 \\
49 \\
48 \\
48 \\
48 \\
48 \\
47\end{array}$ & $\begin{array}{l}41.7 \\
45.4 \\
00.8 \\
01.4 \\
37.2 \\
59.2 \\
34.7 \\
20.8 \\
05.0 \\
42.2\end{array}$ & $\begin{array}{r}5,152 \\
4,587 \\
3,670 \\
4,874 \\
2,221 \\
3,188 \\
2,940 \\
1,753 \\
1,703 \\
3,372\end{array}$ \\
\hline 3 & 56 & 24.8 & 76 & 47 & 10.8 & 5,813 \\
\hline $\begin{array}{l}36 \\
36 \\
36 \\
36\end{array}$ & $\begin{array}{l}56 \\
57 \\
57 \\
58 \\
58 \\
59 \\
59 \\
59\end{array}$ & $\begin{array}{l}53.4 \\
11.5 \\
38.9 \\
15.9 \\
41.4 \\
06.1 \\
16.9 \\
57.1\end{array}$ & $\begin{array}{l}76 \\
76 \\
76 \\
76 \\
76 \\
76 \\
76 \\
76\end{array}$ & $\begin{array}{l}46 \\
46 \\
46 \\
45 \\
45 \\
45 \\
45 \\
45\end{array}$ & $\begin{array}{l}59.2 \\
20.0 \\
04.2 \\
38.3 \\
39.2 \\
46.0 \\
56.9 \\
49.6\end{array}$ & $\begin{array}{l}3,070 \\
3,604 \\
3,054 \\
4,297 \\
2,578 \\
2,561 \\
1,409 \\
4,103\end{array}$ \\
\hline
\end{tabular}


IVOR QUADRANGIE-Continued.

Along highways near southeast corner of quadrangle.

\begin{tabular}{|c|c|c|c|}
\hline Station. & Latitude. & Longitude. & $\begin{array}{l}\text { Distance } \\
\text { between } \\
\text { stations. }\end{array}$ \\
\hline 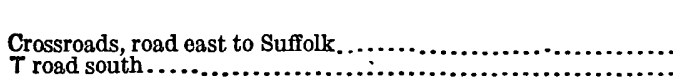 & $\begin{array}{ccc}\circ & \prime & \prime \prime \\
36 & 46 & 06.5 \\
36 & 45 & 45.1\end{array}$ & $\begin{array}{ccc}\circ & \prime & \prime \prime \\
76 & 45 & 02.7 \\
76 & 45 & 10.7\end{array}$ & $\begin{array}{c}\text { Fect. } \\
\cdots \cdots \\
\cdots\end{array}$ \\
\hline
\end{tabular}

Magnetic declination for center of quadrangle, $4.5^{\circ} \mathrm{W}$.; for northeast part of quadrangle, $5.5^{\circ} \mathrm{W}$.

Along highways in northeast part of quadrangle.

Road crossing on narrow-gage railroad.

Stotts Corners, crossroads, Ivor-Smithfield and Isle of wight-

$T$ road through woods, at first decided bend in road from sto

15 feet west of center of lane, at point on line of a long straight

section of road northwest.

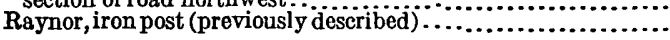

\begin{tabular}{|rrr|rrr|r}
36 & 55 & 18.6 & 76 & 45 & 54.3 & $\ldots \ldots \ldots$ \\
36 & 55 & 34.8 & 76 & 46 & 14.0 & 2,291 \\
& & & & & & \\
36 & 56 & 05.7 & 76 & 46 & 11.5 & \\
36 & 56 & 53.4 & 76 & 46 & 58.2 & 6,133 \\
& & & & & &
\end{tabular}

Northwest along highways near east border of quadrangle.

\begin{tabular}{|c|c|c|c|c|c|c|c|}
\hline 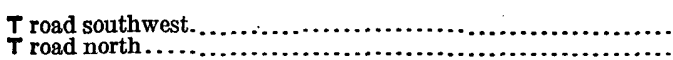 & & $\begin{array}{l}50 \\
51\end{array}$ & $\begin{array}{l}48.2 \\
15.1\end{array}$ & $\begin{array}{l}76 \\
76\end{array}$ & $\begin{array}{l}45 \\
45\end{array}$ & $\begin{array}{l}10.3 \\
13.3\end{array}$ & $\cdots \cdots, \overline{2}, 735$ \\
\hline
\end{tabular}

Northeast along highways near center of quadrangle. (By J. J. Phelan in 1918.)

West side of road, 14 feet from $T$ road to sawmill, 8 feet from mail

box

$T$ road, 9 feetfrom corner fence post.

.

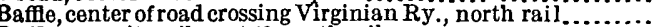

Baffle, op posite milepost 43 , north rail.

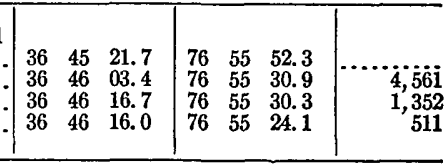

Magnetic declination near south border of quadrangle, $4.2^{\circ} \mathrm{W}$.

JARRATT QUADRANGLE.

In northeast corner of quadrangle. (By J. H. Wilson in 1916-17.)

\begin{tabular}{c|c|ccc}
\hline Station. & Latitude. & Longitude. & $\begin{array}{c}\text { Distance } \\
\text { between } \\
\text { stations. }\end{array}$ \\
\hline $\begin{array}{l}\text { Belcher post office (abandoned), Jerusalem plank road at, at } \\
\text { road northeast, } 720 \text { feet southeast of road southwest, at which } \\
\text { road forks there is an old store building, at center of triangle; } \\
\text { iron post stamped "Prim. Trav. Sta. No. 60, 1917"......... }\end{array}$ & & & & \\
\end{tabular}

South along highways near east border of quadrangle. (By J. C. Fales in 1917-18.)

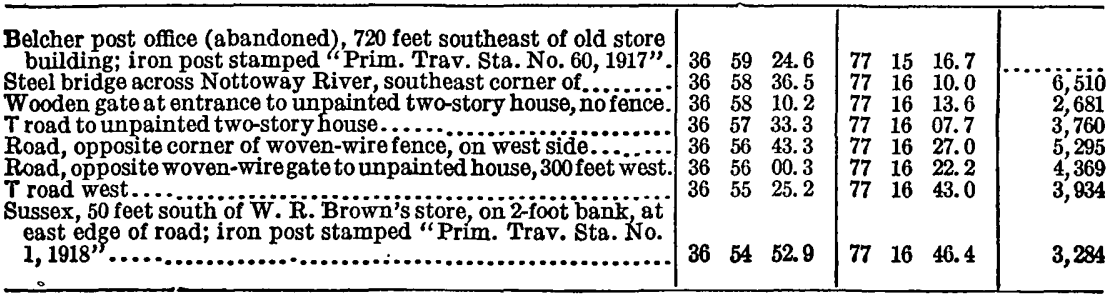


JARRATT QUADRANGLE-Continued.

West along highways through north half of quadrangle.

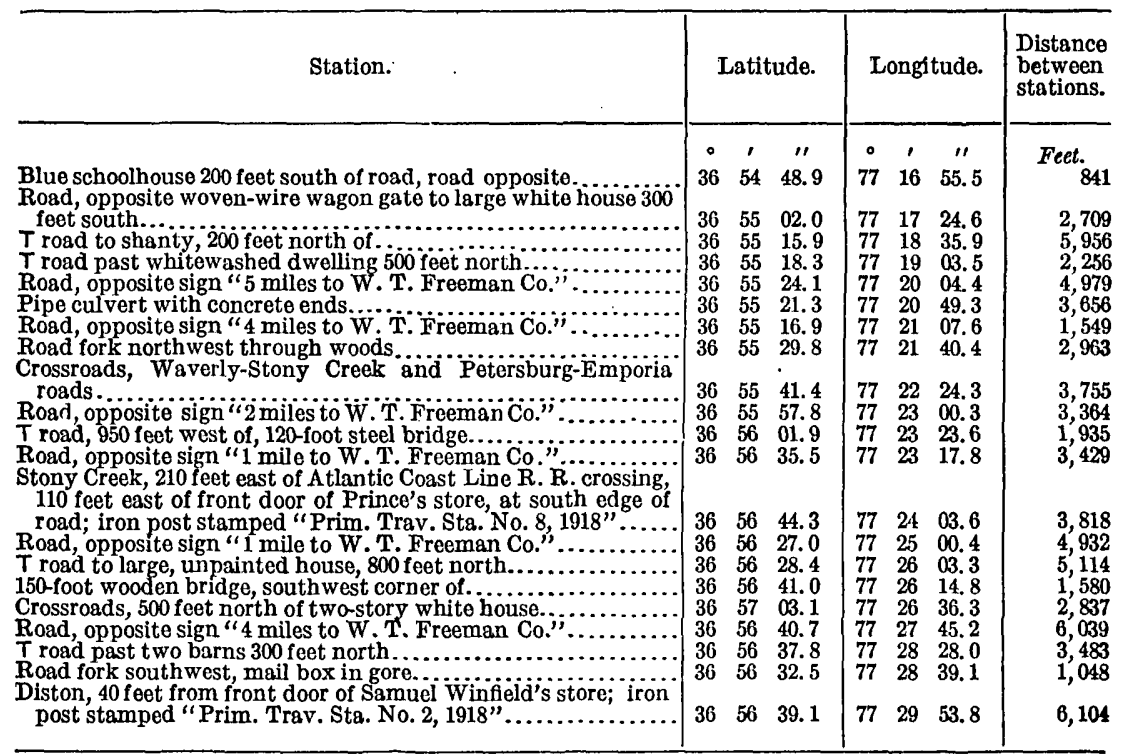

North along Atlantic Coast Line R. R. and highways near west border of quadrangle.

East rail of south-bound track, opposite semaphore 575

East rail of south-bound track, opposite milepost 57

East rail of south-bound track, opposite milepost 56 .

East rail of south-bound track, opposite milepost 55 .

Tast rail of south-bound track, opposite point of switch at

Jarratt, 1,800 feet south of depot, on west side of A tlantic Coast Line tracks; iron post stamped "Prim. Trav. Sta. No. 7, 1918"

Jarratt, road crossing just south of depot, on south-bound track, joint in west rail.

Center of road at wooden.box cuivert

Road forks, wood road north, in center and 6 feet from point of

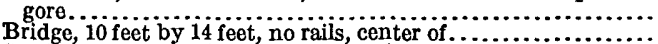

Steel bridge across river, at south end of, center line.

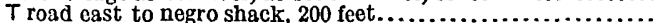
Wooden bridge with rails, 30 by 14 feet, at south end of, center line; white frame church 400 feet southeast.

Forks, woods road northwest.

Road fork to negro dwelling 400 feet west, opposite north point

Road, opposite and 200 feet west of dilapidated dwelling of

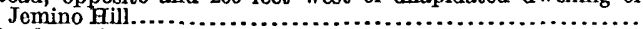

Road crossing.

Road crossing at angle of $75^{\circ}$

\begin{tabular}{|c|c|c|c|c|c|c|}
\hline $\begin{array}{l}36 \\
36 \\
36 \\
36\end{array}$ & $\begin{array}{l}45 \\
45 \\
46 \\
47\end{array}$ & $\begin{array}{l}09.3 \\
43.7 \\
32.8 \\
24.1\end{array}$ & $\begin{array}{l}77 \\
77 \\
77 \\
77\end{array}$ & $\begin{array}{l}29 \\
28 \\
28 \\
28\end{array}$ & $\begin{array}{l}28.8 \\
51.4 \\
29.2 \\
15.4\end{array}$ & $\begin{array}{l}\mathbf{4}, 610 \\
5,285 \\
5,308\end{array}$ \\
\hline 36 & 48 & 11.9 & 77 & 28 & 11.0 & 4,850 \\
\hline 36 & 48 & 32.3 & 77 & 28 & 09.1 & 2,067 \\
\hline $\begin{array}{l}36 \\
36\end{array}$ & $\begin{array}{l}48 \\
49\end{array}$ & $\begin{array}{l}47.9 \\
41.6\end{array}$ & $\begin{array}{l}77 \\
77\end{array}$ & $\begin{array}{l}28 \\
28\end{array}$ & $\begin{array}{l}02.2 \\
47.4\end{array}$ & $\begin{array}{l}1,678 \\
6,560\end{array}$ \\
\hline $\begin{array}{l}36 \\
36 \\
36 \\
36\end{array}$ & $\begin{array}{l}49 \\
50 \\
50 \\
51\end{array}$ & $\begin{array}{l}52.1 \\
27.7 \\
51.0 \\
32.1\end{array}$ & $\begin{array}{l}77 \\
77 \\
77 \\
77\end{array}$ & $\begin{array}{l}28 \\
29 \\
29 \\
29\end{array}$ & $\begin{array}{l}\mathbf{5 5 . 0} \\
\mathbf{1 8 . 4} \\
36.8 \\
50.9\end{array}$ & $\begin{array}{l}1,229 \\
4,072 \\
2,789 \\
4,311\end{array}$ \\
\hline $\begin{array}{l}36 \\
36\end{array}$ & $\begin{array}{l}52 \\
52\end{array}$ & $\begin{array}{l}06.2 \\
40.4\end{array}$ & $\begin{array}{l}77 \\
77\end{array}$ & $\begin{array}{l}29 \\
29\end{array}$ & $\begin{array}{l}45.0 \\
26.0\end{array}$ & $\begin{array}{l}3,481 \\
3,787\end{array}$ \\
\hline 36 & 53 & 24.7 & 77 & 29 & 36.8 & 4,572 \\
\hline $\begin{array}{l}36 \\
36 \\
36\end{array}$ & $\begin{array}{l}53 \\
54 \\
55\end{array}$ & $\begin{array}{l}510 \\
33.8 \\
20.5\end{array}$ & $\begin{array}{l}77 \\
77 \\
77\end{array}$ & $\begin{array}{l}29 \\
29 \\
29\end{array}$ & $\begin{array}{l}42.3 \\
36.6 \\
52.8\end{array}$ & $\begin{array}{l}2,700 \\
4,354 \\
4,896\end{array}$ \\
\hline
\end{tabular}

East along Virginian Ry. from Jarratt to Gray. (By A. C. Schilling in 1918.)
Jarratt, Prim. Trav. Sta. No. 7 (Fales).

Jarratt, crossing of Atlantic Coast Line and Virginian railroads,

south and west rails. . . . . . . . . .

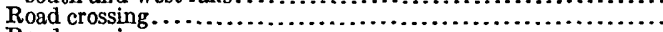

Road crossing.

Milepost 71

Farm-road crossing

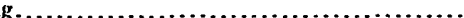

Brown's station, road crossing near. .

Farm-road crossing

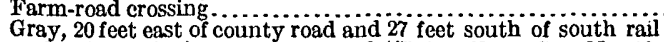
of main track; iron post stamped "Prim. Trav. Sta. No. 16, $1918 "$.

\begin{tabular}{lll|lll|r}
36 & 48 & 32.3 & 77 & 28 & 09.1 & $\ldots \ldots \ldots$ \\
36 & 48 & 56.3 & 77 & 27 & 58.4 & 2,580 \\
36 & 48 & 52.9 & 77 & 27 & 28.1 & 2,492 \\
36 & 48 & 46.6 & 77 & 26 & 32.0 & 4,608 \\
36 & 48 & 36.9 & 77 & 25 & 05.3 & 7,116 \\
36 & 48 & 30.9 & 77 & 24 & 12.6 & 4,328 \\
36 & 48 & 28.9 & 77 & 23 & 54.6 & 1,483 \\
36 & 48 & 15.9 & 77 & 22 & 46.4 & 5,695 \\
36 & 48 & 05.6 & 77 & 22 & 00.0 & 3,917 \\
& & & & & & \\
36 & 47 & 47.3 & 77 & 20 & 39.0 & 6,845 \\
\hline
\end{tabular}


JARRATT QUADRANGLE-Continued.

Northeast from Gray along Southern Ry.

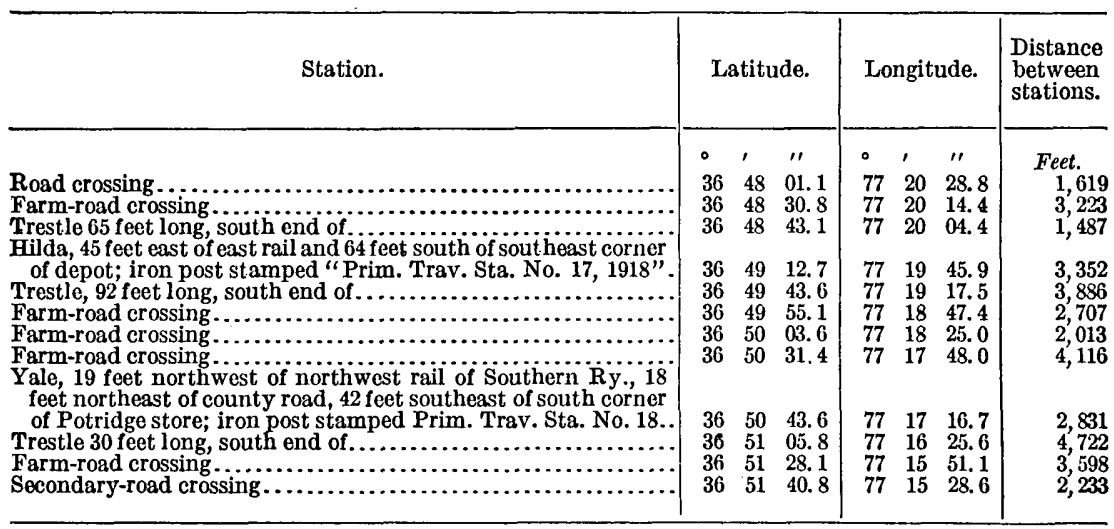

On highway from Sussex to Lumberton.

\begin{tabular}{|c|c|c|c|c|}
\hline $\begin{array}{l}\text { Center line of small bridge (distance from Prim. Trav. Sta. No. } \\
1 \text { at Sussex) } \ldots \ldots \ldots \ldots \ldots \ldots \ldots \ldots \ldots \ldots \ldots \ldots \ldots \ldots\end{array}$ & $\begin{array}{lll}36 & 54 & 29.2\end{array}$ & $77 \quad 1$ & 15.2 & 7,787 \\
\hline
\end{tabular}

South along Atlantic Coast Iine R. R. from Stony Creek to Jarratt.

\begin{tabular}{|c|c|c|c|c|c|c|c|}
\hline 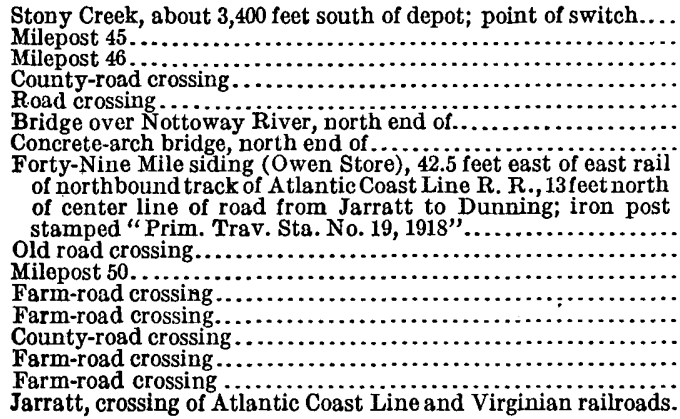 & $\begin{array}{l}36 \\
36 \\
36 \\
36 \\
36 \\
36 \\
36 \\
36 \\
36\end{array}$ & $\begin{array}{l}52 \\
51 \\
51 \\
50 \\
50 \\
49 \\
49 \\
49 \\
48\end{array}$ & $\begin{array}{l}12.8 \\
44.4 \\
18.9 \\
47.3 \\
25.6 \\
57.7 \\
46.2 \\
32.1 \\
56.3\end{array}$ & $\begin{array}{l}77 \\
77 \\
77 \\
77 \\
77 \\
77 \\
77 \\
77 \\
77\end{array}$ & $\begin{array}{l}25 \\
26 \\
26 \\
26 \\
26 \\
26 \\
27 \\
27 \\
27\end{array}$ & $\begin{array}{l}52.7 \\
06.4 \\
18.4 \\
33.0 \\
43.1 \\
57.2 \\
13.2 \\
35.8 \\
58.4\end{array}$ & $\begin{array}{l}2,856 \\
3,078 \\
2,762 \\
3,410 \\
2,332 \\
3,049 \\
1,742 \\
2,324 \\
4,063\end{array}$ \\
\hline
\end{tabular}

Southwest from Gray along Southern Ry.

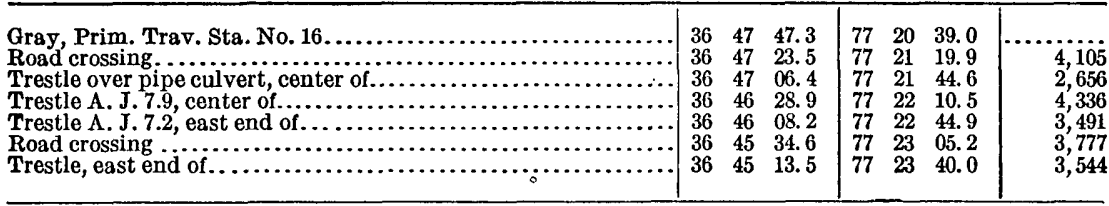

Magnetic declination for west border of quadrangle, $4^{\circ} 20^{\prime} \mathrm{W}$.

Along Virginian Ry. east of Gray.

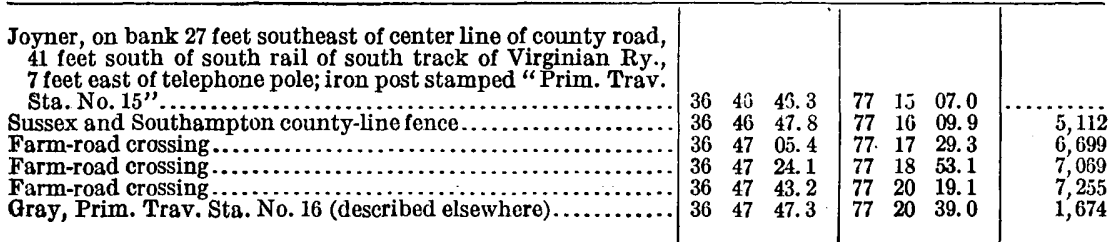


JARRATT QUADRANGLE-Continued.

Along Vlrginlan Ry. west of Jarratt. (By J. C. Fales in 1918.)

\begin{tabular}{|c|c|c|c|}
\hline Station. & Latitude. & Longitude. & $\begin{array}{l}\text { Distance } \\
\text { between } \\
\text { stations. }\end{array}$ \\
\hline 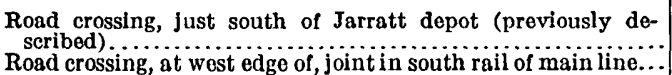 & $\begin{array}{ccc}0 & \prime & \prime \prime \\
36 & 48 & 47.9 \\
36 & 49 & 01.5\end{array}$ & $\begin{array}{ccc}\circ & \prime & \prime \prime \\
77 & 28 & 02.2 \\
77 & 28 & 45.4\end{array}$ & Feet. \\
\hline
\end{tabular}

Northeast along highways near southeast corner of quadrangle. (By H. J. Switzer In 1918.)

Bridgo 69 feet long, south end of .

Culvert of galvanized iron pipe.

Bridge 7 feet long, west end of

Junction of roads.

Bridge 7 feet long, east end of.

(a)

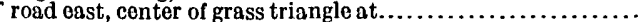

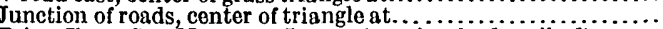

Prim. Trav. Sta. No. 15, at Joyner (proviousiy described).........

\begin{tabular}{|lll|lll|r}
\hline 36 & 45 & 01.1 & 77 & 18 & 41.7 & $\ldots \ldots \ldots$ \\
36 & 45 & 01.9 & 77 & 18 & 00.9 & $3,32 j$ \\
36 & 45 & 06.0 & 77 & 16 & 50.2 & 5,767 \\
36 & 45 & 16.9 & 77 & 15 & 51.9 & 4,864 \\
36 & 46 & 00.1 & 77 & 16 & 02.0 & 4,448 \\
36 & 46 & 19.9 & 77 & 15 & 58.4 & 2,023 \\
36 & 46 & 39.6 & 77 & 16 & 06.3 & 2,091 \\
36 & 46 & 46.3 & 77 & 15 & 07.0 & 4,867
\end{tabular}

Magnetic declination for southeast corner of quadrangle, $4^{\circ} 30^{\prime} \mathrm{W}$.

Northwest along highways in northwest part of quadrangle, (By J. C. Fales in 1918.)

Crossroads (position previously given)

Road fork, 800 feet south of unpainted farm building .............

$T$ road west past whitewashed negro cabin 400 feet distant

$T$ road, 150 feet north of whitewashed negro cabin, mail box

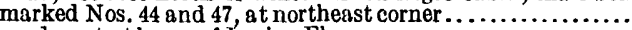

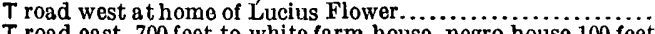

$T$ road east, 700 feet to white farm house, negro house 100 fee

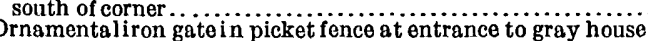

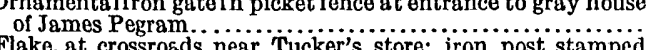

Flake, at crossroads near Tucker's store; iron post stamped

"Prim. Trav. Sta. No. 3, 1918" ........................

\begin{tabular}{|rrr|rrr|r}
36 & 57 & 03.1 & 77 & 26 & 36.3 & $\ldots \ldots \ldots$ \\
36 & 57 & 30.8 & 77 & 26 & 59.1 & 3,359 \\
36 & 57 & 41.7 & 77 & 27 & 08.2 & 1,320 \\
36 & 57 & 59.7 & 77 & 27 & 17.5 & 1,976 \\
36 & 58 & 20.5 & 77 & 28 & 15.0 & 5,113 \\
36 & 58 & 48.2 & 77 & 28 & 36.8 & 3,310 \\
36 & 59 & 01.0 & .77 & 29 & 23.4 & 3,996 \\
36 & 59 & 40.5 & 77 & 29 & 06.6 & 4,221 \\
\hline
\end{tabular}

Magnetic declination for northwest part of quadrangle, $4^{\circ} 10^{\prime} \mathrm{W}$.

KARA QUADRANGLE.

Along Seaboard Air Line Ry. near southeast corner of quadrangle. (By J. C. Fales In 1918.)

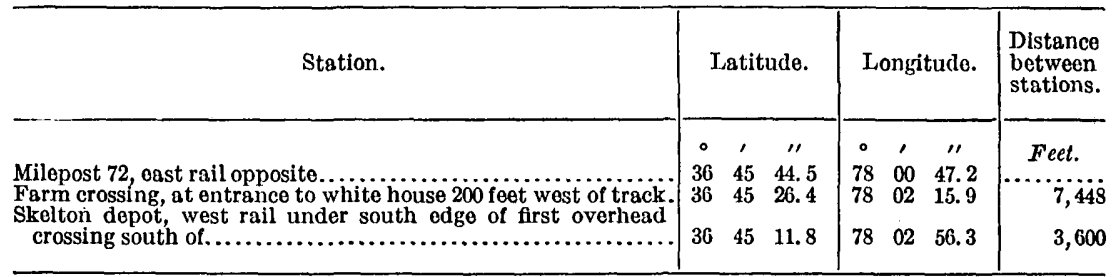

KILMARNOCK QUADRANGLE.

(By E. L. McNair In 1916.)

\begin{tabular}{|c|c|c|c|}
\hline Station. & Latitude. & I.ongitude. & $\begin{array}{l}\text { Distance } \\
\text { hetween } \\
\text { stations. }\end{array}$ \\
\hline 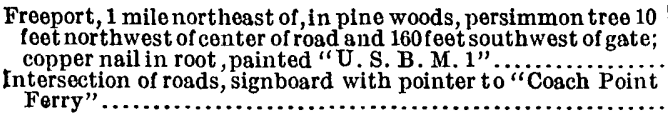 & $\begin{array}{ccc}\circ & \prime & \prime \prime \\
37 & 32 & 53.5 \\
37 & 33 & 38.9\end{array}$ & $\begin{array}{ccc}\circ & \prime & \prime \prime \\
76 & 29 & 36.5 \\
76 & 29 & 09.1\end{array}$ & $\begin{array}{r}\text { Feet. } \\
5,098\end{array}$ \\
\hline
\end{tabular}

Magnetic declination for south half of west side of quadrangle, $5^{\circ} 59^{\prime} \mathrm{W}$. 
KING WILIIAM QUADRANGLE.

North along highways near east border of north half of quadrangle. (By E. L. McNair in 1916.)

\begin{tabular}{|c|c|c|c|c|c|c|c|}
\hline Station. & \multicolumn{3}{|c|}{ Latitude. } & \multicolumn{3}{|c|}{ Longitude. } & $\begin{array}{l}\text { Distance } \\
\text { between } \\
\text { stations. }\end{array}$ \\
\hline 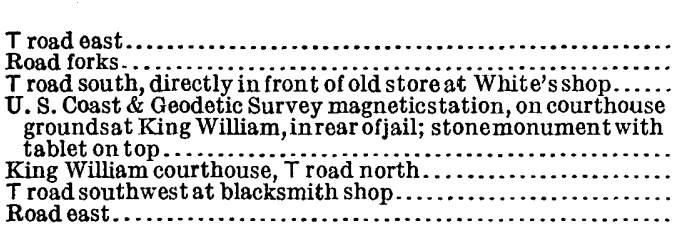 & $\begin{array}{r}\circ \\
37 \\
37 \\
37 \\
\\
\\
37 \\
37 \\
37 \\
37\end{array}$ & $\begin{array}{c}\prime \\
39 \\
40 \\
40 \\
\\
41 \\
41 \\
41 \\
42\end{array}$ & $\begin{array}{c}\prime \prime \\
39.9 \\
05.6 \\
47.4 \\
\\
17.1 \\
13.4 \\
39.1 \\
52.9\end{array}$ & $\begin{array}{c}\circ \\
77 \\
77 \\
77 \\
\\
77 \\
77 \\
77 \\
77\end{array}$ & $\begin{array}{l}\prime \\
00 \\
00 \\
00 \\
\\
\\
00 \\
00 \\
01 \\
01\end{array}$ & $\begin{array}{c}\prime \prime \\
00.5 \\
26.2 \\
20.2 \\
\\
46.1 \\
50.0 \\
57.9 \\
51.6\end{array}$ & \begin{tabular}{c} 
Feet. \\
\hdashline 3,322 \\
4,267
\end{tabular} \\
\hline
\end{tabular}

Magnetic declination for east border of quadrangle, $5^{\circ} 35^{\prime} \mathrm{W}$.

Along highways in the northeast quarter of quadrangle.

\begin{tabular}{|c|c|c|c|c|c|c|c|}
\hline 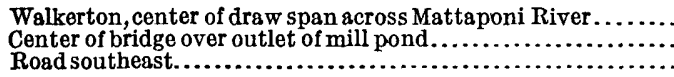 & $\begin{array}{l}37 \\
37 \\
37\end{array}$ & $\begin{array}{l}43 \\
43 \\
44\end{array}$ & $\begin{array}{l}22.6 \\
41.9 \\
13.3\end{array}$ & $\begin{array}{l}77 \\
77 \\
77\end{array}$ & $\begin{array}{l}01 \\
01 \\
00\end{array}$ & $\begin{array}{l}33.0 \\
13.6 \\
21.9\end{array}$ & $\begin{array}{r}\ddot{2}, 495 \\
5,227\end{array}$ \\
\hline
\end{tabular}

Magnetic declination for northeast quarter of quadrangle, $5^{\circ} 50^{\prime} \mathrm{W}$.

East along highways near north border of quadrangle.

\begin{tabular}{|c|c|c|c|c|c|c|c|}
\hline 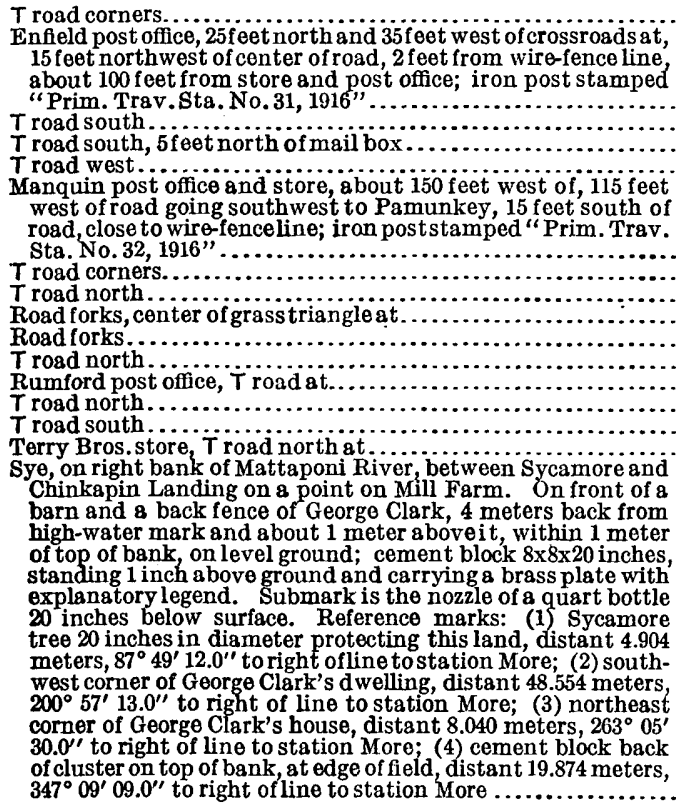 & $\begin{array}{l}37 \\
37 \\
37 \\
37 \\
37 \\
37 \\
37 \\
37 \\
37 \\
37\end{array}$ & $\begin{array}{l}\mathbf{4 2} \\
42 \\
42 \\
43 \\
43 \\
43 \\
43 \\
43 \\
43 \\
43\end{array}$ & $\begin{array}{l}32.3 \\
47.5 \\
14.5 \\
20.0 \\
41.5 \\
34.4 \\
28.8 \\
17.0 \\
06.8 \\
02.5\end{array}$ & $\begin{array}{l}77 \\
77 \\
77 \\
77 \\
77 \\
77 \\
77 \\
77 \\
77 \\
77\end{array}$ & $\begin{array}{l}09 \\
08 \\
07 \\
06 \\
06 \\
05 \\
05 \\
04 \\
04 \\
03\end{array}$ & $\begin{array}{l}16.2 \\
44.4 \\
11.6 \\
50.0 \\
02.4 \\
31.0 \\
23.0 \\
58.9 \\
29.0 \\
49.9\end{array}$ & $\begin{array}{r}\mathbf{6}, 416 \\
2,979 \\
8,164 \\
6,843 \\
4,400 \\
2,625 \\
858 \\
2,270 \\
2,619 \\
3,168\end{array}$ \\
\hline
\end{tabular}

Magnetic declination for north border of quadrangle, $5^{\circ} 00^{\prime} \mathrm{W}$. 
KING WILIIAM QUADRANGLE-Continued.

Along highways near west border of quadrangle.

\begin{tabular}{|c|c|c|c|}
\hline Station. & Latitude. & Longitude. & $\begin{array}{l}\text { Distance } \\
\text { between } \\
\text { stations. }\end{array}$ \\
\hline 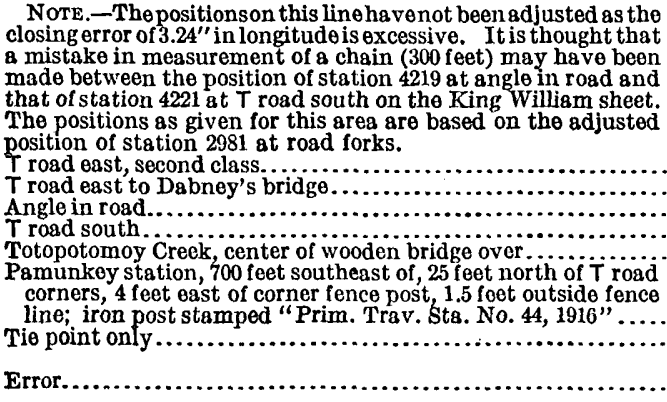 & $\begin{array}{ccc}\circ & , & \prime \prime \\
37 & \mathbf{4 1} & 50.8 \\
37 & 41 & 07.1 \\
37 & 40 & 55.2 \\
37 & 41 & 02.3 \\
37 & \mathbf{4 0} & 46.9 \\
& & \\
& & \\
37 & 39 & 48.2 \\
37 & 38 & 39.29 \\
37 & \mathbf{3 8} & \mathbf{3 8 . 8 1} \\
& & +.48\end{array}$ & $\begin{array}{ccc}\circ & \prime & \prime \prime \\
77 & 14 & 42.6 \\
77 & 14 & 42.9 \\
77 & 14 & 08.8 \\
77 & 13 & 31.9 \\
77 & 13 & 05.0 \\
& & \\
& & \\
77 & 11 & 42.7 \\
77 & 12 & 03.84 \\
77 & 12 & 07.08 \\
& & -3.24\end{array}$ & $\begin{array}{r}\text { Feet. } \\
\ldots \ldots \\
4,414 \\
\cdots \ldots . . . \\
2,659 \\
\\
8,889 \\
7,173 \\
\ldots \ldots \ldots\end{array}$ \\
\hline
\end{tabular}

Along highways from center to west border of quadrangle.

\begin{tabular}{|c|c|c|c|c|c|c|c|}
\hline 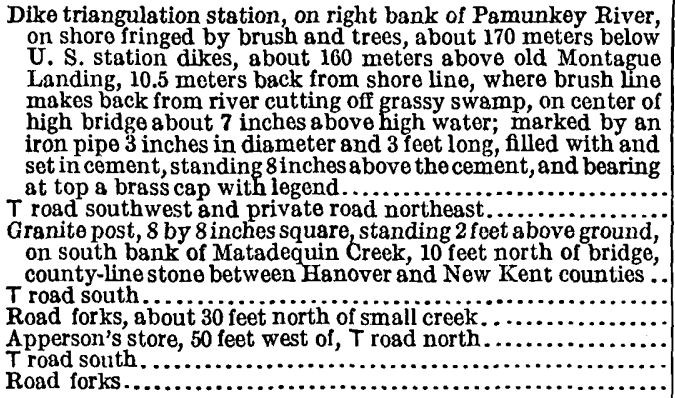 & $\begin{array}{l}37 \\
37 \\
37 \\
37 \\
37 \\
37\end{array}$ & $\begin{array}{l}37 \\
36 \\
\\
\\
36 \\
37 \\
38 \\
38 \\
38 \\
38\end{array}$ & $\begin{array}{l}20.07 \\
44.7 \\
\\
59.4 \\
37.1 \\
09.9 \\
35.1 \\
32.9 \\
31.6\end{array}$ & $\begin{array}{l}77 \\
77 \\
77 \\
77 \\
77 \\
77\end{array}$ & $\begin{array}{l}08 \\
08 \\
09 \\
10 \\
11 \\
11\end{array}$ & $\begin{array}{l}16.04 \\
49.7 \\
\\
15.7 \\
39.3 \\
57.9 \\
32.0 \\
04.8 \\
44.0\end{array}$ & $\begin{array}{r}8,344 \\
2,567 \\
4,253 \\
7,141 \\
3,742 \\
2,648 \\
3,158\end{array}$ \\
\hline
\end{tabular}

South along highways near south hatf of west border of quadrangle.

Old Church post office, T road south at.

Matadequin Creek, center of wooden bridge over...............

$T$ road southwest.

Creek crossing, small wooden bridge over

old Church post office, 3.1 miles south of, 28 feet south and

10 feet east of crassroáds, opposite house on southwest corner of crossroads, just inside field, 2 feet from corner fence post;

iron post stamped "Prim. Trav. Sta. No. 45, 1916" ............

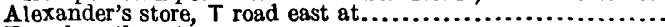

$T$ road southwest.

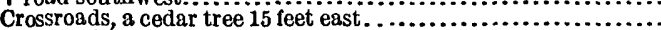

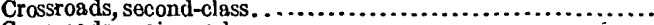

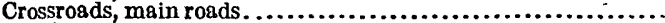

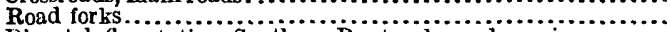

Dispatch flag station, Southern Ry. track, road crossing........

Rosd forks, about 40 feet northeast of pine tree at point of

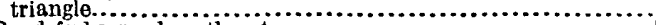

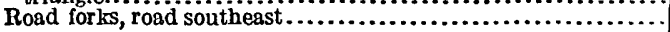

\begin{tabular}{|lll|lll|l}
37 & 38 & 40.3 & 77 & 13 & $\mathbf{1 7 . 2}$ & $\mathbf{5 , 6 4 5}$ \\
37 & 37 & 54.0 & 77 & 13 & 24.9 & 4,722 \\
37 & 37 & 18.5 & 77 & 13 & 28.3 & 3,606 \\
37 & 36 & 42.7 & 77 & 13 & 12.8 & 3,831 \\
& & & & & & \\
& & & & & & \\
37 & 36 & 05.8 & 77 & 13 & 06.6 & 3,761 \\
37 & 35 & 11.4 & 77 & 13 & 23.4 & 5,669 \\
37 & 34 & 42.4 & 77 & 13 & 33.8 & 3,056 \\
37 & 33 & 59.2 & 77 & 13 & 33.0 & 4,371 \\
37 & 33 & 16.9 & 77 & 12 & 52.0 & 5,406 \\
37 & 32 & 57.6 & 77 & 12 & 30.6 & 2,597 \\
37 & 32 & 37.6 & 77 & 12 & 14.4 & 2,414 \\
37 & 31 & 52.1 & 77 & 11 & 53.8 & 4,897 \\
37 & 31 & 26.6 & 77 & 10 & 56.4 & $\ldots \ldots, \ldots$ \\
37 & 31 & 40.9 & 77 & 10 & 26.5 & 2,810 \\
\hline
\end{tabular}


RING WILLIAM QUADRANGLE-Continued.

East along highways near south border of quadrangle.

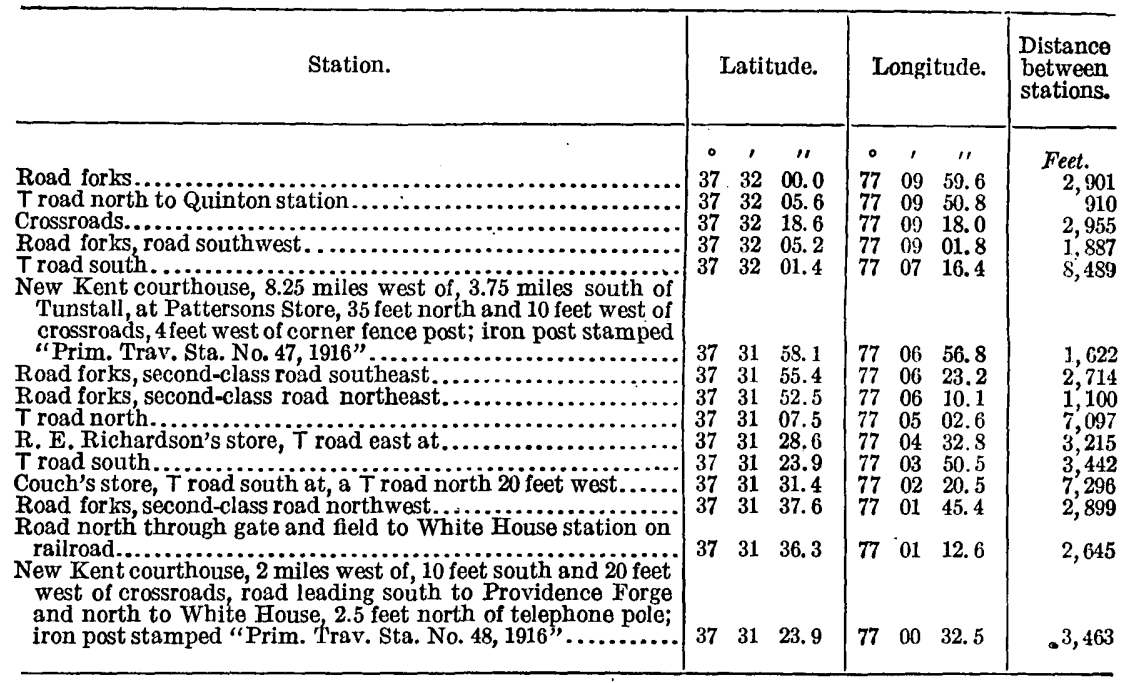

Magnetic declination for center line of quadrangle (east and west), $4^{\circ} 10^{\circ} \mathrm{W}$.; for west border, $4^{\circ} 40^{\prime} \mathrm{W}$.

Along Richmond and Norfolk highway in southwest corner of quadrangle. (By E. I. MacNair in 1916.)

Road northwest

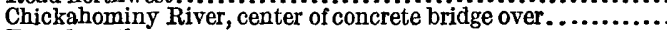

Troad south.

Road forks, road west............................................

Road forks, about 35 feet west and 10 feet south of

signboard, about 12 feet west of Lexington Hotel signboard; iron post stamped "Prim. Trav. Sta. No. 46, 1916".

\begin{tabular}{|lll|lll|r}
37 & 31 & 07.0 & 77 & 11 & 33.1 & $\ldots \ldots \ldots$ \\
37 & 30 & 51.7 & 77 & 12 & 26.3 & 4,551 \\
37 & 30 & 37.0 & 77 & 12 & 39.4 & \\
37 & 30 & 42.0 & 77 & 13 & 53.3 & \\
& & & & & & \\
37 & 30 & 29.2 & 77 & 14 & 33.0 & \\
\end{tabular}

Along highways in southeast corner of quadrangle. (By J. H. Wilson in 1916.)

Troad south.

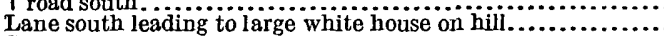

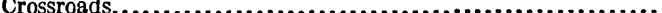

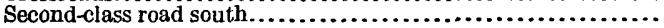

\begin{tabular}{|lll|lll|r}
37 & 30 & 05.0 & 77 & 02 & 13.0 & $\ldots \ldots \ldots \ldots$ \\
37 & 30 & 23.2 & 77 & 01 & 49.3 & 2,653 \\
37 & 30 & 29.8 & 77 & 01 & 28.7 & 1,794 \\
37 & 30 & 49.7 & 77 & 00 & 42.0 & 4,268 \\
\hline
\end{tabular}

Magnetic declination in south part of quadrangle, $5^{\circ} 03^{\prime} \mathrm{W}$.

\section{LAWRENCEVILLE QUADRANGLE.}

South a long highways near southeast corner of quadrangle. (By J. C. Fales in 1918.)

\begin{tabular}{|c|c|c|c|c|c|c|c|}
\hline - & \multicolumn{3}{|c|}{ Latitude. } & \multicolumn{3}{|c|}{ Iongitude. } & $\begin{array}{c}\begin{array}{c}\text { Distance } \\
\text { between } \\
\text { stations. }\end{array} \\
\text { Feet. }\end{array}$ \\
\hline 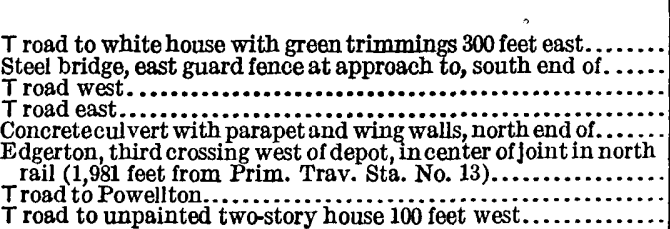 & $\begin{array}{l}36 \\
36 \\
36 \\
36 \\
36 \\
36 \\
36 \\
36\end{array}$ & $\begin{array}{l}1 \\
49 \\
48 \\
48 \\
48 \\
47 \\
46 \\
46 \\
45\end{array}$ & $\begin{array}{l}03.2 \\
42.1 \\
25.8 \\
00.4 \\
09.8 \\
43.3 \\
11.8 \\
23.2\end{array}$ & $\begin{array}{c}0 \\
77 \\
77 \\
77 \\
77 \\
77 \\
77 \\
77 \\
77\end{array}$ & $\begin{array}{c}\prime \\
45 \\
45 \\
45 \\
46 \\
46 \\
\\
46 \\
47 \\
47\end{array}$ & $\begin{array}{c}\prime \prime \\
10.2 \\
36.2 \\
53.6 \\
05.2 \\
31.9 \\
\\
42.3 \\
28.2 \\
05.7\end{array}$ & $\begin{array}{r}\text { Feet. } \\
\ldots \ldots \ldots . . . \\
3,005 \\
2,172 \\
2,735 \\
5,557 \\
\\
2,811 \\
4,908 \\
5,237\end{array}$ \\
\hline
\end{tabular}


IAWRETCEVILLE QUADRATGLE-Continued.

Along Southern Ry. east from Edgerton.

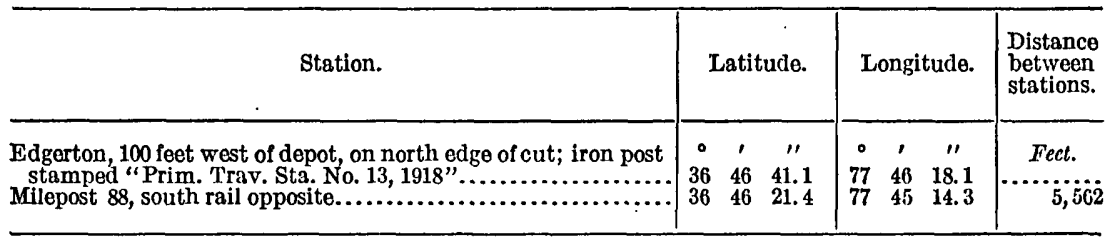

Magnetic declination for southeast part of quadrangle, $4^{\circ} 10^{\prime} \mathrm{W}$.

Along Southem Ry. west from Edgerton. (By J. C. Fales in 1918.)

Edgerton, third road crossing west of depot, in center of, joint in

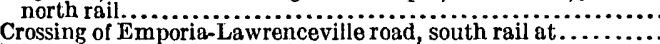

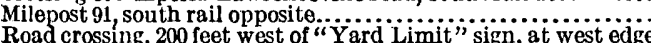
Road crossing, 200 feet west of "Yard Limit" sign, at west edge

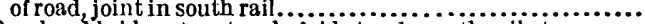

Overhead bridge at east end of side track, south rail at.......... Lawrenceville, at crossing sign, 60 feet east of freight house; iron post stamped "Prim. Trav. Sta. No. 16, 1918" ..............

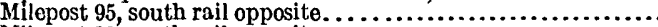

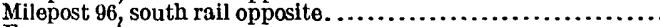

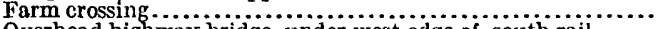

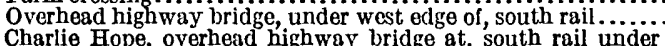

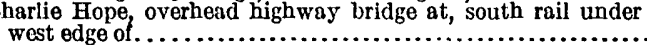

\begin{tabular}{|lll|lll|r}
36 & 46 & 43.3 & 77 & 46 & 42.3 & $\ldots \ldots$ \\
36 & 46 & 50.8 & 77 & 47 & 41.0 & 4,883 \\
36 & 46 & 49.8 & 77 & 48 & 21.5 & 3,293 \\
36 & 45 & 59.9 & 77 & 49 & 25.4 & 7,247 \\
36 & 45 & 23.7 & 77 & 50 & 51.0 & 7,875 \\
36 & 45 & 22.9 & 77 & 51 & 03.3 & 1,007 \\
36 & 45 & 36.3 & 77 & 51 & 54.6 & 4,387 \\
36 & 45 & 39.8 & 77 & 52 & 59.4 & 5,284 \\
36 & 46 & 02.2 & 77 & 53 & 42.0 & 4,141 \\
36 & 46 & 01.3 & 77 & 54 & 25.8 & 3,558 \\
36 & 45 & 24.1 & 77 & 55 & 25.6 & 6,159 \\
\hline
\end{tabular}

Southwest along Seaboard Air Line Ry. through center of quadrangle.

Nottoway River, stoel truss across, east rail at south end of.....

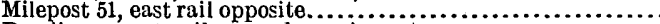

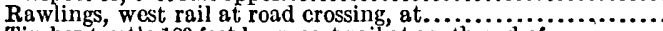

Timber trestle 180 feet long, east rail at south ond of.

Farm-road crossing, second joint in east rail south of, 338 feet

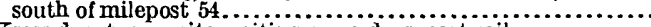

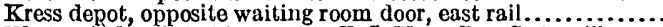

West switch point at turnout to F. J. Kress Box Co.'s mill......

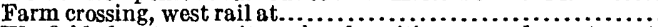
Warfield depot, opposite colored waiting-room door at west

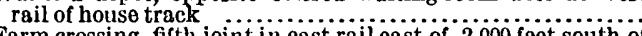
Farm crossing, firth joint in east rail east of, 2,000 feet south of

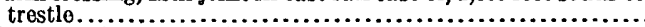

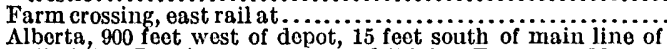
Alberta, 900 feet west of depot, 15 feet south of main line of
Virginian Ry.; iron post stamped "Prim. Trav. Sta. No. 20, Virginian Ry.;iron post stamped "Prim. Trav. Sta. No. 20,

Alberta, overhead girder bridge for virginian $\mathrm{Ry}$. crossing, under north edge of, west rail of Seaboard Air Line Ry...... Trarm crossing, east rail at . . . . . . . . . . . . . . . . . . . . . . . .

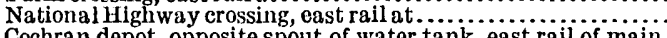
Cochran depot, opposite spout of water tank, east rail of main

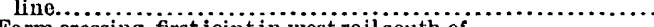

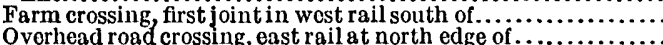

Overhead road crossing, east rail at north edge of .........................

Grandy depot, about 600 feet southwest of, opposite milepost 68 .

Overhead road crossing, west rail under north edge of............

National Highway crossing, 1,830 feet east of milopost 70 i6

feet south of track; iron post stamped "Prim. Trav. Sta.

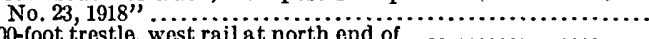

300 -foot trestle, west rail at north end of . . . . . . . . . . . . . . . . .

\begin{tabular}{|c|c|c|c|c|c|c|}
\hline $\begin{array}{l}36 \\
36 \\
36 \\
36\end{array}$ & $\begin{array}{l}57 \\
57 \\
56 \\
56\end{array}$ & $\begin{array}{l}22.5 \\
13.6 \\
54.1 \\
29.4\end{array}$ & $\begin{array}{l}77 \\
77 \\
77 \\
77\end{array}$ & $\begin{array}{l}45 \\
45 \\
45 \\
47\end{array}$ & $\begin{array}{l}03.3 \\
32.0 \\
44.5 \\
49.5\end{array}$ & $\begin{array}{l}2,498 \\
6,209 \\
5,836\end{array}$ \\
\hline $\begin{array}{l}36 \\
36 \\
36 \\
36\end{array}$ & $\begin{array}{l}56 \\
55 \\
55 \\
54\end{array}$ & $\begin{array}{l}07.2 \\
42.9 \\
25.4 \\
32.9\end{array}$ & $\begin{array}{l}77 \\
77 \\
77 \\
77\end{array}$ & $\begin{array}{l}48 \\
48 \\
49 \\
49\end{array}$ & $\begin{array}{l}21.8 \\
48.1 \\
01.8 \\
14.0\end{array}$ & $\begin{array}{l}3,451 \\
3,257 \\
2,085 \\
5,401\end{array}$ \\
\hline 36 & 53 & 43.5 & 77 & 49 & 36.6 & 5,320 \\
\hline $\begin{array}{l}36 \\
36\end{array}$ & $\begin{array}{l}52 \\
52\end{array}$ & $\begin{array}{l}41.7 \\
31.9\end{array}$ & $\begin{array}{l}77 \\
77\end{array}$ & $\begin{array}{l}50 \\
51\end{array}$ & $\begin{array}{l}37.9 \\
34.4\end{array}$ & $\begin{array}{l}7,988 \\
4,698\end{array}$ \\
\hline 36 & 51 & 53.8 & 77 & 53 & 08.6 & 8,573 \\
\hline $\begin{array}{l}36 \\
36 \\
36\end{array}$ & $\begin{array}{l}51 \\
51 \\
50\end{array}$ & $\begin{array}{l}50.5 \\
13.4 \\
33.5\end{array}$ & $\begin{array}{l}77 \\
77 \\
77\end{array}$ & $\begin{array}{l}53 \\
53 \\
54\end{array}$ & $\begin{array}{l}01.8 \\
49.5 \\
20.3\end{array}$ & $\begin{array}{r}650 \\
5,399 \\
4,752\end{array}$ \\
\hline $\begin{array}{l}36 \\
36 \\
36 \\
36 \\
36 \\
36\end{array}$ & $\begin{array}{l}49 \\
49 \\
49 \\
47 \\
47 \\
47\end{array}$ & $\begin{array}{l}57.7 \\
43.9 \\
03.1 \\
56.9 \\
46.7 \\
05.2\end{array}$ & $\begin{array}{l}77 \\
77 \\
77 \\
77 \\
77 \\
77\end{array}$ & $\begin{array}{l}54 \\
55 \\
56 \\
57 \\
57 \\
58\end{array}$ & $\begin{array}{l}40.0 \\
22.6 \\
08.4 \\
08.2 \\
45.4 \\
04.3\end{array}$ & $\begin{array}{l}3,953 \\
3,740 \\
5,557 \\
8,270 \\
3,195 \\
4,474\end{array}$ \\
\hline $\begin{array}{l}36 \\
36 \\
36\end{array}$ & $\begin{array}{l}46 \\
46 \\
46\end{array}$ & $\begin{array}{l}42.2 \\
29.1 \\
05.8\end{array}$ & $\begin{array}{l}77 \\
77 \\
77\end{array}$ & $\begin{array}{l}58 \\
59 \\
59\end{array}$ & $\begin{array}{l}45.0 \\
14.2 \\
56.5\end{array}$ & $\begin{array}{l}4,048 \\
2,719 \\
4,166\end{array}$ \\
\hline
\end{tabular}

$\ddot{2}, 49 \ddot{8}$

6,209

3,451

3,257

2,085

5,326

7,988

4,698

8,573

650

5,399
4,752

3,953

, 740

5,557
8,270

3,195

4,474

4,048

4,166

West along Virginian Ry. through center of quadrangle.

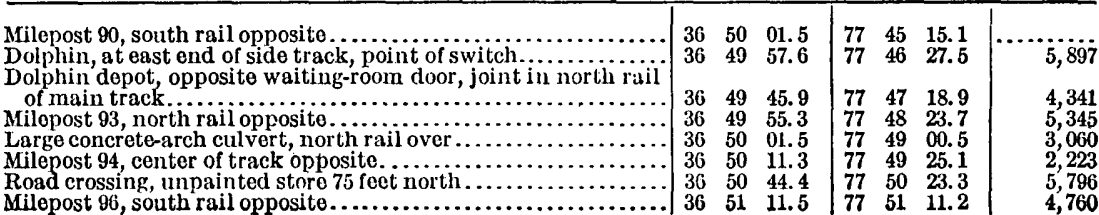


LAWRENCEVILLE QUADRANGLE-Continued.

West along Virginian Ry. through center of quadrangle-Continued.

\begin{tabular}{|c|c|c|c|}
\hline - Station. & Latitude. & Longitude. & $\begin{array}{l}\text { Distance } \\
\text { between } \\
\text { stations. }\end{array}$ \\
\hline 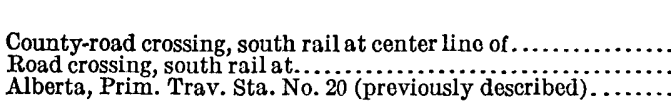 & $\begin{array}{rrr}\circ & \prime & \prime \prime \\
36 & 51 & 27.5 \\
36 & 51 & 47.8 \\
36 & 51 & 53.8\end{array}$ & $\begin{array}{ccc}\circ & , & \prime \prime \\
77 & 51 & 39.4 \\
77 & 52 & 20.6 \\
77 & 53 & 08.6\end{array}$ & $\begin{array}{l}\text { Feet. } \\
2,801 \\
3,924 \\
3,944\end{array}$ \\
\hline
\end{tabular}

(By J. C. Fales In 1918.)

\begin{tabular}{|c|c|c|c|c|c|c|c|}
\hline 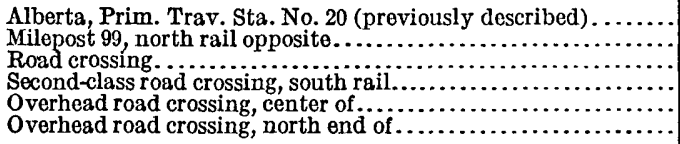 & $\begin{array}{l}36 \\
36 \\
36 \\
36 \\
36 \\
36\end{array}$ & $\begin{array}{l}51 \\
52 \\
52 \\
53 \\
53 \\
54\end{array}$ & $\begin{array}{l}53.8 \\
08.1 \\
31.8 \\
18.7 \\
57.4 \\
11.0\end{array}$ & $\begin{array}{l}77 \\
77 \\
77 \\
77 \\
77 \\
77\end{array}$ & $\begin{array}{l}53 \\
54 \\
55 \\
56 \\
56 \\
57\end{array}$ & $\begin{array}{l}08.6 \\
08.7 \\
05.2 \\
06.1 \\
58.2 \\
56.2\end{array}$ & $\begin{array}{l}3,096 \\
5,175 \\
6,829 \\
5,794 \\
4,907\end{array}$ \\
\hline
\end{tabular}

North along highways near west border of quadrangle.

Danieltown, at southeast corner of crossroads, at store and post office; iron post stamped "Prim. Trav. Sta. No. 21, 1918" Road fork, northeast toward sawmill

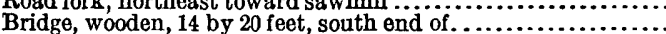

Road forks, middle of south leg of $Y$.

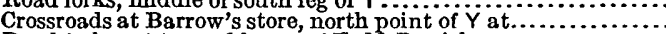

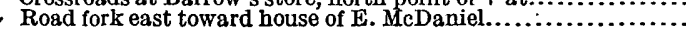

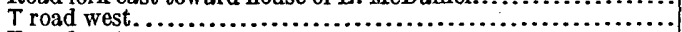

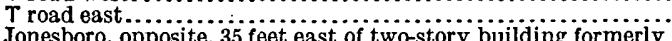

Jonesboro, opposite, 35 feet east of two-story building formerly

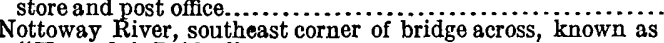

" "Kettoway River, southeast corner of bridge across, known as

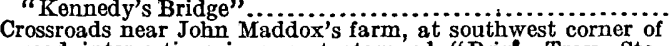
road intersection; iron post stamped "Prim. Trav. Sta. No. 22, 1918" "

\begin{tabular}{|lll|lll|l} 
& & & & & & \\
36 & 54 & 16.0 & 77 & 57 & 31.9 & 2,031 \\
36 & 54 & 49.7 & 77 & 57 & 42.6 & 3,515 \\
36 & 55 & 17.4 & 77 & 58 & 0.3 & 3,489 \\
36 & 55 & 50.8 & 77 & 58 & 05.8 & 3,378 \\
36 & 56 & 06.3 & 77 & 58 & 44.7 & 3,530 \\
36 & 56 & 47.1 & 77 & 58 & 46.0 & 4,126 \\
36 & 57 & 14.8 & 77 & 58 & 24.2 & 3,319 \\
36 & 57 & 55.4 & 77 & 57 & 59.7 & 4,559 \\
36 & 58 & 43.5 & 77 & 57 & 41.8 & 5,079 \\
36 & 59 & 21.3 & 77 & 57 & 47.2 & 3,845 \\
& & & & & & \\
36 & 59 & 58.1 & 77 & 57 & 57.3 & 3,819 \\
\hline
\end{tabular}

East along highways near north border of quadrangle.

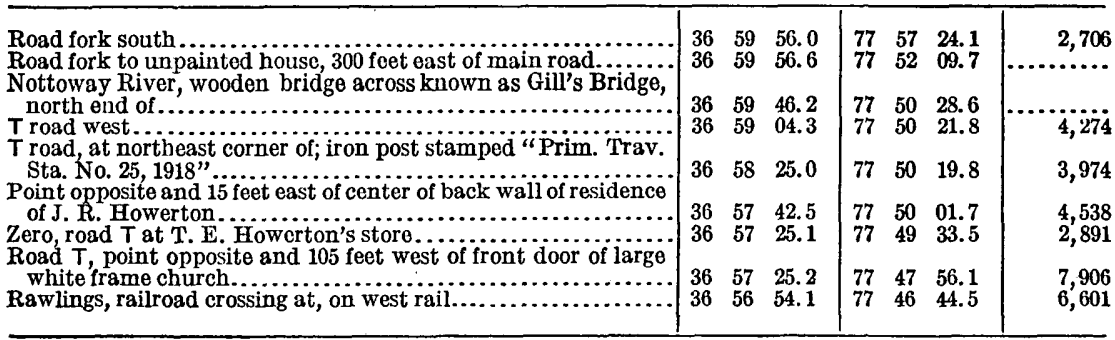

North along highways from Grandy to Tobacco near west border of quadrangle.

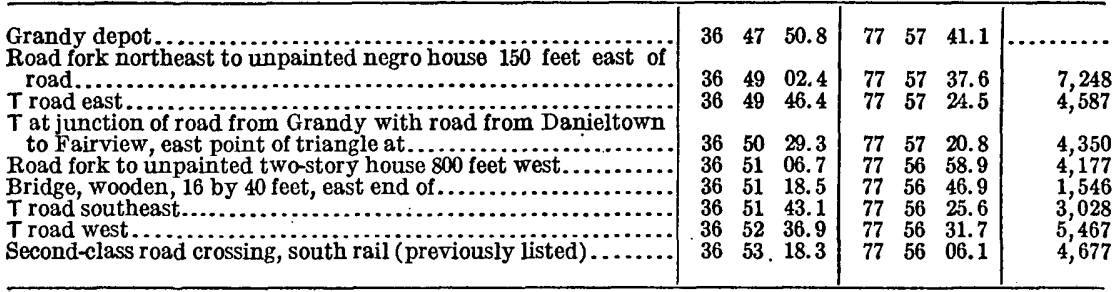

Magnetic declination for north border of quadrangle, $4^{\circ} 17^{\prime} \mathrm{W}$.; for west border of guadrangle, $3^{\circ} 51^{\prime} \mathrm{W}$. 
MCKENNEY QUADRANGLE.

West along Virginian Ry. through south half of quadrangle. (By J. C. Fales in 1918.)

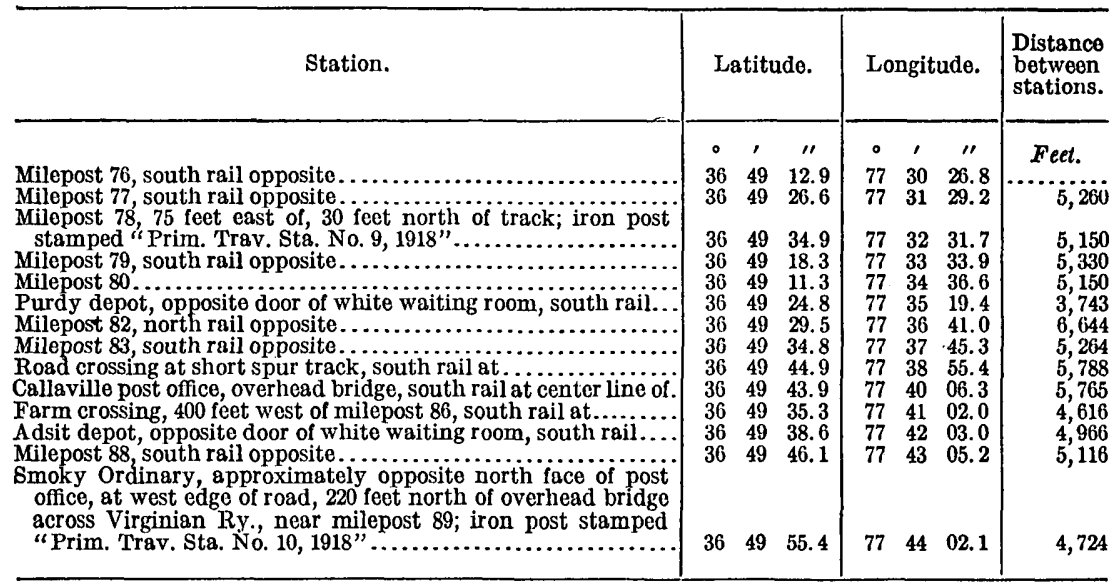

West along Southern Ry. near south border of quadrangle.

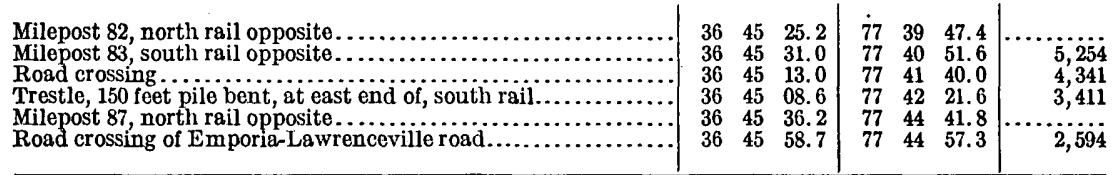

Along highways near east border of quadrangle.

\begin{tabular}{|c|c|c|c|c|c|c|c|}
\hline 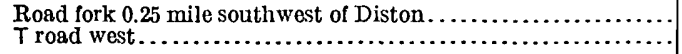 & $\begin{array}{l}36 \\
36\end{array}$ & $\begin{array}{l}56 \\
55\end{array}$ & $\begin{array}{l}28.5 \\
53.8\end{array}$ & $\begin{array}{l}77 \\
77\end{array}$ & $\begin{array}{l}30 \\
30\end{array}$ & $\begin{array}{l}08.4 \\
01.2\end{array}$ & $\cdots \cdots$ \\
\hline
\end{tabular}

West along highways through north half of quadrangle. (By J. C. Fales in 1918.)

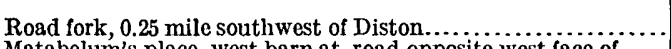
Matabelum's place, west barn at, road opposite west face of...... Truitt, 0.75 mile east of, fork west.

Truitt, county road, 85 feet north of north face of $\mathrm{M}_{\text {. }} \mathbf{L}$. $\mathrm{Rose}$ 's store at.

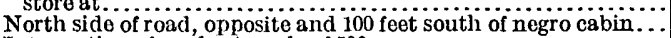
Intersection of roads at angle of $50^{\circ}$

$T$ road west...

Two-story house with exterior chimney 100 fect north of road.

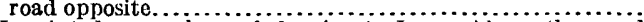

Unpainted negro house belonging to James A bernathy, road

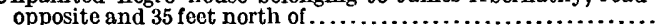
Unused log barn, road opposite and 50 feet north of . . . . . . . . . . .

Crossroads 75 feet south of church ........................... road

30-foot $\mathbf{I}$-beam bridge with pipe railings, east end of, at center

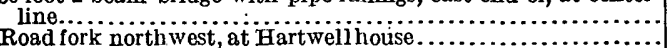
Road fork northwest, at Hartwell house.................................. Clark's store, opposite and 40 feet west of front door of .

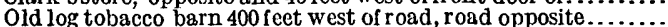

Log stable 200 f eet east of road, road opposite southwest corner of. Crossroads, 500 feet east of tobacco warehouse.

Picket wagon gate to white house 400 feet west, road opposite.... Crossroad from schoolhouse, 300 feet east to white dwelling, 700

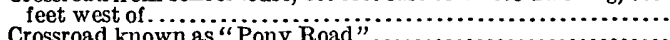

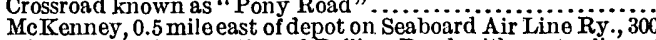

feet west of intersection of Bolling Road with center line of Boydtown Plank Road; iron post stamped "Prim. Trav. Ste. No. 11, 1918"

\begin{tabular}{|c|c|c|c|c|c|c|}
\hline $\begin{array}{l}36 \\
36 \\
36\end{array}$ & $\begin{array}{l}56 \\
56 \\
55\end{array}$ & $\begin{array}{l}28.5 \\
23.5 \\
57.9\end{array}$ & $\begin{array}{l}77 \\
77 \\
77\end{array}$ & $\begin{array}{l}30 \\
31 \\
32\end{array}$ & $\begin{array}{l}08.4 \\
04.5 \\
10.1\end{array}$ & $\begin{array}{r}4,595 \\
5,918\end{array}$ \\
\hline $\begin{array}{l}36 \\
36 \\
36 \\
36\end{array}$ & $\begin{array}{l}56 \\
56 \\
55 \\
55\end{array}$ & $\begin{array}{l}07.0 \\
13.2 \\
58.0 \\
38.2\end{array}$ & $\begin{array}{l}77 \\
77 \\
77 \\
77\end{array}$ & $\begin{array}{l}33 \\
33 \\
33 \\
34\end{array}$ & $\begin{array}{l}00.7 \\
37.4 \\
52.3 \\
08.8\end{array}$ & $\begin{array}{l}4,213 \\
3,046 \\
1,947 \\
2,411\end{array}$ \\
\hline 36 & 55 & 33.3 & 77 & 35 & 05.3 & 4,617 \\
\hline $\begin{array}{l}36 \\
36 \\
36\end{array}$ & $\begin{array}{l}55 \\
55 \\
55\end{array}$ & $\begin{array}{l}42.7 \\
46.9 \\
44.8\end{array}$ & $\begin{array}{l}77 \\
77 \\
77\end{array}$ & $\begin{array}{l}35 \\
36 \\
37\end{array}$ & $\begin{array}{l}45.1 \\
41.1 \\
05.8\end{array}$ & $\begin{array}{l}3,372 \\
4,565 \\
2,017\end{array}$ \\
\hline 36 & 56 & 11.2 & 77 & 37 & 24.9 & 3,086 \\
\hline $\begin{array}{l}36 \\
36 \\
36 \\
36 \\
36 \\
36 \\
36 \\
36\end{array}$ & $\begin{array}{l}\mathbf{5 5} \\
\mathbf{5 5} \\
\mathbf{5 4} \\
\mathbf{5 4} \\
\mathbf{5 5} \\
\mathbf{5 5} \\
56 \\
\mathbf{5 7}\end{array}$ & $\begin{array}{l}32.8 \\
19.9 \\
53.1 \\
44.4 \\
36.3 \\
55.6 \\
49.7 \\
32.6\end{array}$ & $\begin{array}{l}77 \\
77 \\
77 \\
77 \\
77 \\
77 \\
77 \\
77\end{array}$ & $\begin{array}{l}38 \\
39 \\
39 \\
40 \\
40 \\
40 \\
40 \\
41\end{array}$ & $\begin{array}{l}18.7 \\
16.2 \\
46.8 \\
05.3 \\
24.7 \\
33.0 \\
54.6 \\
23.1\end{array}$ & $\begin{array}{l}5,846 \\
4,854 \\
3,666 \\
1,746 \\
5,482 \\
2,064 \\
5,751 \\
4,915\end{array}$ \\
\hline $\begin{array}{l}36 \\
36\end{array}$ & $\begin{array}{l}58 \\
58\end{array}$ & $\begin{array}{l}01.4 \\
14.0\end{array}$ & $\begin{array}{l}77 \\
77\end{array}$ & $\begin{array}{l}41 \\
42\end{array}$ & $\begin{array}{l}56.7 \\
07.6\end{array}$ & $\begin{array}{l}3,988 \\
1,550\end{array}$ \\
\hline 36 & 58 & 57.6 & 77 & 43 & 01.0 & 6,181 \\
\hline
\end{tabular}


MCKENREY QUADRANGLE-Continued.

Along Seaboard Air Line Ry. northeast from McKenney.

\begin{tabular}{|c|c|c|c|}
\hline Station. & Latitude. & Longitude. & $\begin{array}{l}\text { Distance } \\
\text { between } \\
\text { stations. }\end{array}$ \\
\hline 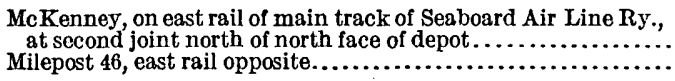 & $\begin{array}{ccc}\circ & \prime & \prime \prime \\
36 & 59 & 18.4 \\
36 & 59 & 58.2\end{array}$ & $\begin{array}{ccc}\circ & \prime & \prime \prime \\
77 & 43 & 21.5 \\
77 & 42 & 09.2\end{array}$ & $\begin{array}{r}\text { Feet. } \\
2,683 \\
7,106\end{array}$ \\
\hline
\end{tabular}

North along highways near west border of quadrangle.

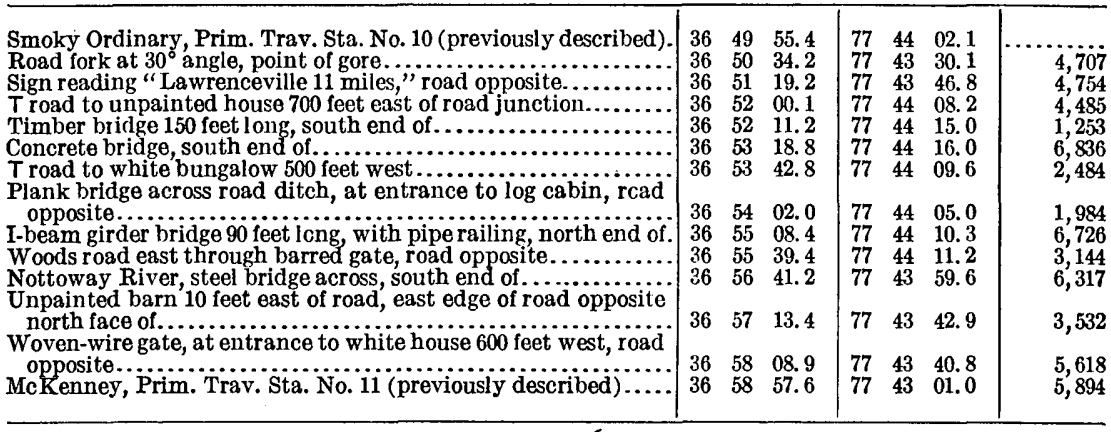

Magnetic declination for north half of quadrangle, $4^{\circ} 10^{\prime} \mathrm{W}$; for west border of quadrangle, $4^{\circ} 20^{\prime} \mathrm{W}$.

Along Seaboard Air Line Ry. near west border of quadrangle. (By J. C. Fates in 1918.)

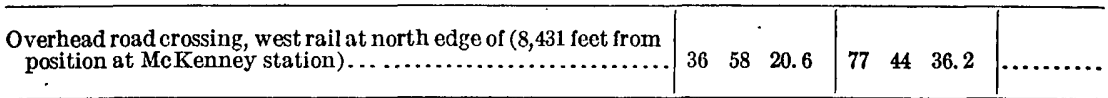

\section{MONTROSS QUADRANGLE.}

Along highways near southeast corner of quadrangle. (By E. L. McNair in 1916.)

\begin{tabular}{|c|c|c|c|}
\hline Station. & Latitude. & Longitude. & $\begin{array}{l}\text { Distance } \\
\text { between }\end{array}$ \\
\hline 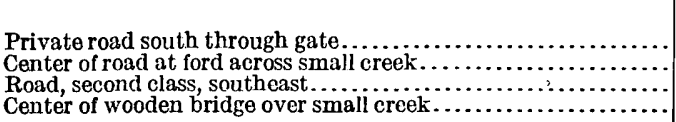 & $\begin{array}{ccc}\circ & \prime & \prime \prime \\
38 & 00 & 26.8 \\
38 & 00 & 32.6 \\
38 & 00 & 50.1 \\
38 & 00 & 03.0\end{array}$ & $\begin{array}{ccc}\circ & , & \prime \prime \\
76 & 45 & 07.7 \\
76 & 45 & 35.8 \\
76 & 46 & 02.9 \\
76 & 46 & 24.4\end{array}$ & $\begin{array}{c}F e e_{c}^{\prime} \\
(4,721) \\
2,325 \\
2,799 \\
5,059\end{array}$ \\
\hline
\end{tabular}

Along highways near south border of quadrangle (east half).

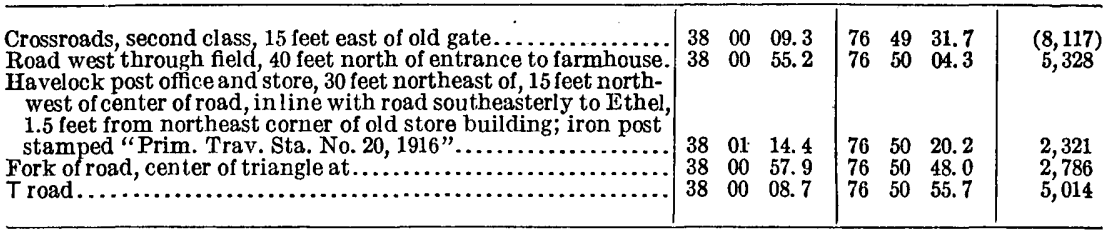

Magnetic declination along border near southeast corner of quadrangle, $6^{\circ} 10 \mathrm{~W}$. 
MORATTICO QUADRANGLE.

North along highways near east border of quadrangle. (By E $/$ L. McNalr in 1916.)

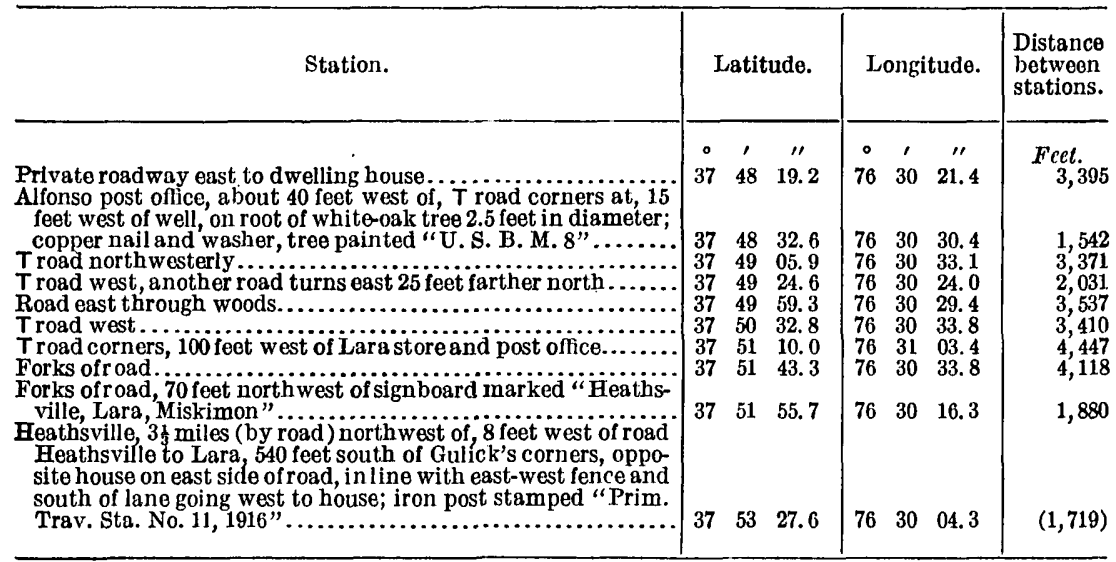

Magnetic declination for east border of quadrangle, $5^{\circ} 38^{\prime} \mathrm{W}$.

Along highways in southeast corner of quadrangle. (Checked spur line.)

Lively post office, crossroads at

Crossroads, second class

Rosd forks second lass..................

Beanes Mill, center of road over mill race

Bethel M. E. Church South, center of road in front of...........

\begin{tabular}{|lll|rrr|r}
37 & 46 & 39.7 & 76 & 30 & 50.0 & $(5,060)$ \\
37 & 46 & 16.9 & 76 & -31 & 14.0 & 3,012 \\
37 & 45 & 47.3 & 76 & 31 & 42.4 & 3,755 \\
37 & 45 & 32.7 & 76 & 32 & 10.8 & 2,722 \\
37 & 45 & 08.5 & 76 & 32 & 32.9 & 3,024
\end{tabular}

Magnetic declination in southeast corner of quadrangle, $5^{\circ} 40^{\prime} \mathrm{W}$.

Northwest along highways near northeast corner of quadrangle.

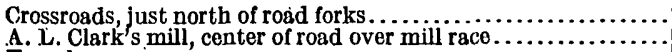

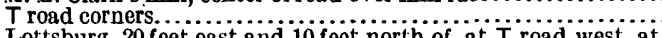
Lottsburg, 20 feet east and 10 feet north of, at T road west, at north end of private hedge; iron post stamped "Prim. Trav. Sto No 13, 1916"

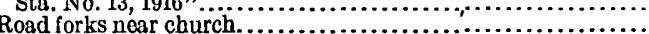
$T$ road northeast.

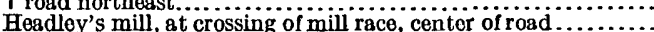

Callao, 10 feet south and 40 feet east of crossroads at, 18 feet east of center of road, 3 feet from telephone pole; iron post stamped "Prim. Trav.'Sta. No. 14, 1916"...........................

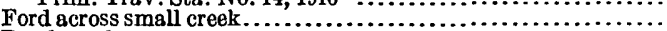
Road south.

Hyacinth post office, road forks, 100 feet south of

\begin{tabular}{|c|c|c|c|c|c|c|}
\hline $\begin{array}{l}37 \\
37 \\
37\end{array}$ & $\begin{array}{l}56 \\
56 \\
56\end{array}$ & $\begin{array}{l}08.0 \\
48.7 \\
52.9\end{array}$ & $\begin{array}{l}76 \\
76 \\
76\end{array}$ & $\begin{array}{l}30 \\
30 \\
31\end{array}$ & $\begin{array}{l}15.2 \\
25.8 \\
00.7\end{array}$ & $\begin{array}{r}(2,613) \\
\mathbf{4}, 203 \\
2,828\end{array}$ \\
\hline 37 & $\begin{array}{l}57 \\
57 \\
58 \\
58\end{array}$ & $\begin{array}{l}26.9 \\
50.3 \\
07.2 \\
10.3\end{array}$ & $\begin{array}{l}76 \\
76 \\
76 \\
76\end{array}$ & $\begin{array}{l}31 \\
31 \\
31 \\
32\end{array}$ & $\begin{array}{l}05.7 \\
13.4 \\
43.3 \\
31.4\end{array}$ & $\begin{array}{l}3,455 \\
2,453 \\
2,942 \\
3,863\end{array}$ \\
\hline $\begin{array}{l}37 \\
37\end{array}$ & $\begin{array}{l}58 \\
58 \\
58 \\
59 \\
00\end{array}$ & $\begin{array}{l}10.3 \\
31.5 \\
31.8 \\
24.9 \\
00.1\end{array}$ & $\begin{array}{l}76 \\
76 \\
76 \\
76 \\
76\end{array}$ & $\begin{array}{l}\mathbf{3 3} \\
\mathbf{3 4} \\
\mathbf{3 4} \\
\mathbf{3 5} \\
\mathbf{3 4}\end{array}$ & $\begin{array}{l}35.8 \\
21.5 \\
44.5 \\
23.9 \\
57.8\end{array}$ & $\begin{array}{l}\mathbf{5}, 154 \\
4,249 \\
1,843 \\
6,227 \\
4,129\end{array}$ \\
\hline
\end{tabular}

Along highways near northwest corner of quadrangle.

Private roads at bend in main road.

$T$ road corners, at road south.

Road north second class

Lyells post office and store, $T$ road east at.......................

\begin{tabular}{|lll|lll|r}
37 & 59 & 46.7 & 76 & 40 & 49.2 & $(6,025)$ \\
37 & 59 & 36.6 & 76 & 41 & 12.5 & 2,129 \\
37 & 59 & 33.4 & 76 & 41 & 56.7 & 3,558 \\
37 & 59 & 42.9 & 76 & 42 & 43.1 & 3,830 \\
37 & 59 & 50.3 & 76 & $\mathbf{4 3}$ & $\mathbf{4 3 . 6}$ & 4,877 \\
\hline
\end{tabular}

Along highways near west border of quadrangle.

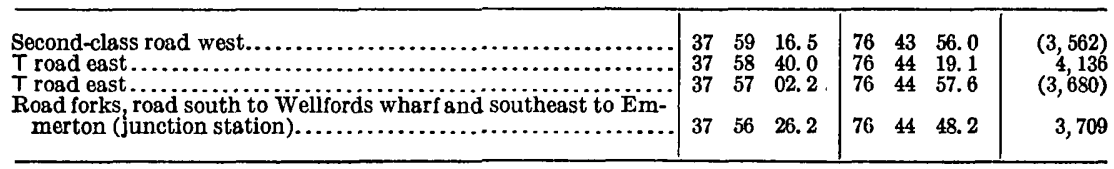


MORATTICO QUADRANGLE-Continued.

Checked spur line along highways near west border of quadrangle.

Station.

Along hlghways west to east through center of quadrangle.

\begin{tabular}{|c|c|c|c|c|c|c|c|}
\hline 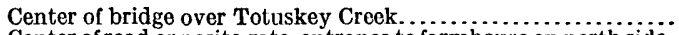 & 37 & 55 & 24.5 & 76 & 43 & 15.5 & $(9,702)$ \\
\hline Center of road opposite gate, entrance to farmhouse on north side & & & & & 42 & & \\
\hline 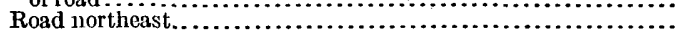 & $\begin{array}{l}51 \\
37\end{array}$ & 54 & 25.4 & $\begin{array}{l}10 \\
76\end{array}$ & 42 & 07.1 & 5,674 \\
\hline 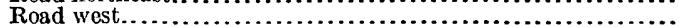 & 37 & 54 & 03.6 & 76 & 40 & 57.8 & 2,325 \\
\hline T road north at Emmertion........... & 37 & 53 & 56.4 & 76 & 40 & 47.9 & 1,075 \\
\hline 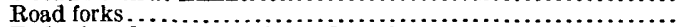 & 37 & 53 & 27.8 & 76 & 40 & 15.2 & 3,906 \\
\hline Fork of roads near church, road southeast..................... & 37 & 53 & 22.9 & 76 & 39 & 57.2 & 1,520 \\
\hline $\begin{array}{l}\text { Farnham post office, } 0.8 \text { mile west of, at corner where road goes } \\
\text { west to Sharps, } 15 \text { feet south of Emmerton-Farnham road and } \\
20 \text { feet east of road to Sharps, } 20 \text { feet from corner fence post; }\end{array}$ & & & 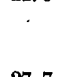 & & & & \\
\hline iron post stamped "Prim. Trav. Sta. No. 18, 1916" $\ldots \ldots \ldots \ldots$. & 37 & 53 & 27.7 & 76 & 38 & & 8,040 \\
\hline 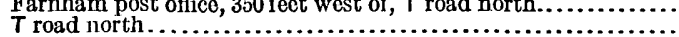 & $\begin{array}{l}37 \\
37\end{array}$ & $\begin{array}{l}53 \\
52\end{array}$ & $\begin{array}{l}09.6 \\
59.6\end{array}$ & $\begin{array}{l}76 \\
76\end{array}$ & $\begin{array}{l}37 \\
36\end{array}$ & $\begin{array}{l}33.3 \\
24.6\end{array}$ & $\begin{array}{l}3,964 \\
5,601\end{array}$ \\
\hline Road north... & 37 & 52 & 47. 3 & 76 & 35 & 41.2 & 3,697 \\
\hline T road south ............. & 37 & 52 & 38.1 & 76 & 35 & 13.5 & 2,404 \\
\hline Private road to house & 37 & 52 & 00.9 & 76 & 34 & 28.7 & 5,200 \\
\hline $\begin{array}{l}\text { Farnham, } 3 \text { miles southcast of, } 12 \text { feet southeast of center of road, } \\
\text { in root of pine tree; copper nail and washer, painted " } U \text {. S. }\end{array}$ & & & & & & & \\
\hline В. М. $11 ", \ldots \ldots$ & 37 & 51 & 49.0 & 76 & 34 & 02.6 & 2,410 \\
\hline Center of Lara-Farnham road, in line with road south...... & 37 & 51 & 46.4 & 76 & 33 & 52.5 & 862 \\
\hline Road forks, center of grass triangle at. $\ldots \ldots \ldots \ldots \ldots \ldots \ldots$ & 37 & 52 & 10.9 & 76 & 33 & 21.5 & 3,511 \\
\hline Road forks, road north about 100 feet east of road south... & 37 & 52 & 08.2 & 76 & 33 & 00.8 & 1,686 \\
\hline Road forks, about 0.6 mile north of camp grounds...... & 37 & 53 & 05.6 & 76 & 32 & 28.6 & 6,349 \\
\hline Crossroads, second-class road west through woods... & 37 & 53 & 31.5 & 76 & 32 & 33.0 & 2,646 \\
\hline$T$ road corners, road east. $\ldots \ldots \ldots \ldots \ldots \ldots \ldots \ldots$ & 37 & 54 & 08.5 & 76 & 32 & 41.0 & 3,794 \\
\hline Road forks, 15 feet east of signboard...... & 37 & 53 & 50.6 & 76 & 31 & 49.2 & 4,527 \\
\hline Road forks, 30 feet southeast of signboard. . . . . . . . . . . . . . . & 37 & 54 & 03.7 & 76 & 31 & 07.9 & 3,564 \\
\hline 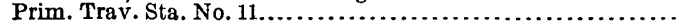 & 37 & 53 & 27.6 & 76 & 30 & 04.3 & 6,278 \\
\hline
\end{tabular}

Magnetic declination near northeast corner of quadrangle, $5^{\circ} 45^{\prime} \mathrm{W}$.; near northwest corner of quadrangle, $5^{\circ} 54^{\prime} \mathrm{W}$.; near west border of quadrangle, $6^{\circ} 10^{\prime} \mathrm{W}$.; west to east through center of quadrangle, $5^{\circ} 40^{\prime} \mathrm{W}$.

Along highways across extreme southwest corner of quadrangle.

\begin{tabular}{|c|c|c|c|c|c|c|}
\hline 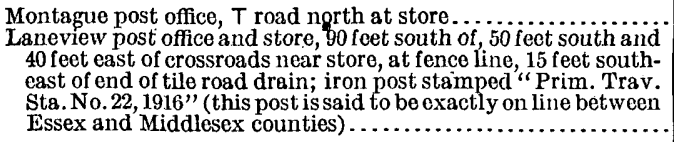 & & 46 & 05.1 & $\begin{array}{ll}76 \quad 44 \\
\end{array}$ & 14.3 & $(4,021)$ \\
\hline
\end{tabular}

Along highways near south border of quadrangle.

\begin{tabular}{|c|c|c|c|c|c|c|c|}
\hline $\begin{array}{l}\text { T road northwest, second class. } \\
\text { Butylo post office, at crossing of Middlesex and Essex county }\end{array}$ & & 46 & 16.6 & & $4 l$ & 39.7 & 9,711 \\
\hline 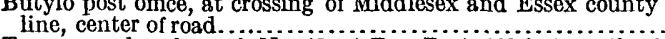 & 37 & 46 & 09.3 & 76 & 41 & 12.8 & 2,280 \\
\hline $\begin{array}{l}\text { Temporary bench mark No. } 13 \text {, at Bay Port, } 100 \text { feet south of } \\
\text { store and post office, } 20 \text { feet west of road, at edge of timber; } \\
\text { copper nail in root of gum tree, painted "U. }\end{array}$ & & & & & & & \\
\hline 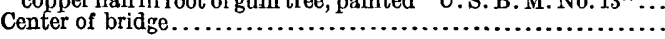 & 37 & 45 & $\begin{array}{l}\text { 15. } \\
02.7\end{array}$ & 76 & $\begin{array}{ll}40 \\
40\end{array}$ & $\begin{array}{l}26.9 \\
17.7\end{array}$ & $\begin{array}{l}6,585 \\
1,469\end{array}$ \\
\hline
\end{tabular}

Magnetic declination for extreme southwest corner of quadrangle, $5^{\circ} 40^{\prime} \mathrm{W}$.; magnetic declination near south border of quadrangle, $5^{\circ} 35^{\prime} \mathrm{W}$. 
MOYOCK QUADRANGLE.

South along Norfolk Southern R. R. near northwest corner of quadrangle. (By F. J. McMaugh in 1917.)

\begin{tabular}{l} 
Station. \\
\hline
\end{tabular}

NEW KENT QUADRANGIE.

Near Dudley's ferry. (By. E. L. McNair in 1016.)

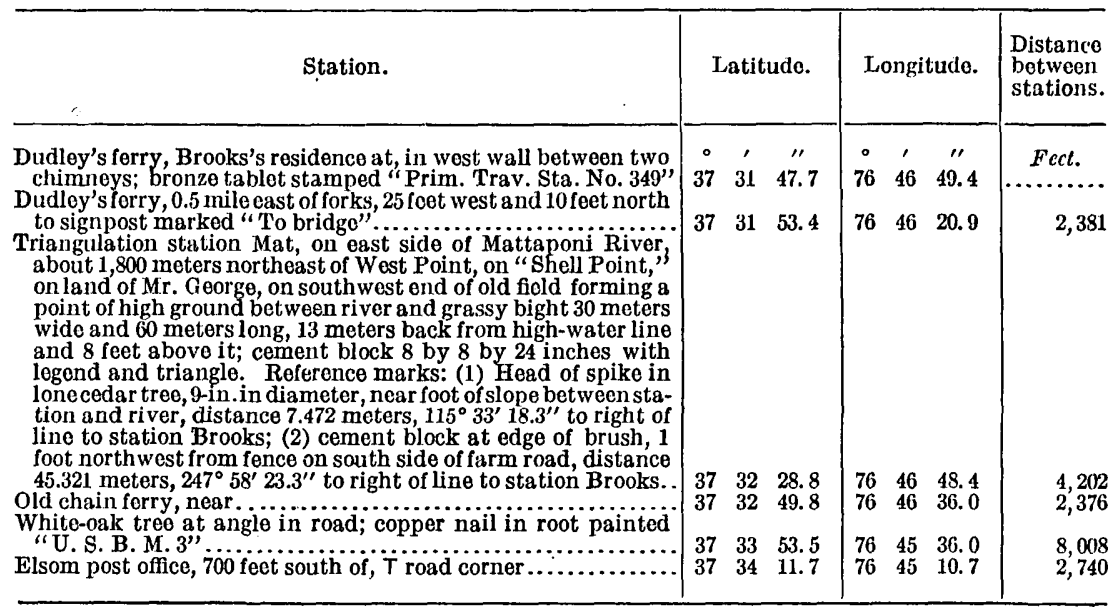

Magnetic declination for south half of east side of quadrangle, $5^{\circ} 24^{\prime} \mathrm{W}$.

Near east border of quadrangle.

\begin{tabular}{|c|c|c|c|}
\hline Road forks, about 600 feet southeast of Wares Church.. & $\begin{array}{lll}37 & 40 & 49.1\end{array}$ & $\begin{array}{lll}76 & 45 & 04.5\end{array}$ & $(7,160)$ \\
\hline
\end{tabular}

Along highways near west border of quadrangle.

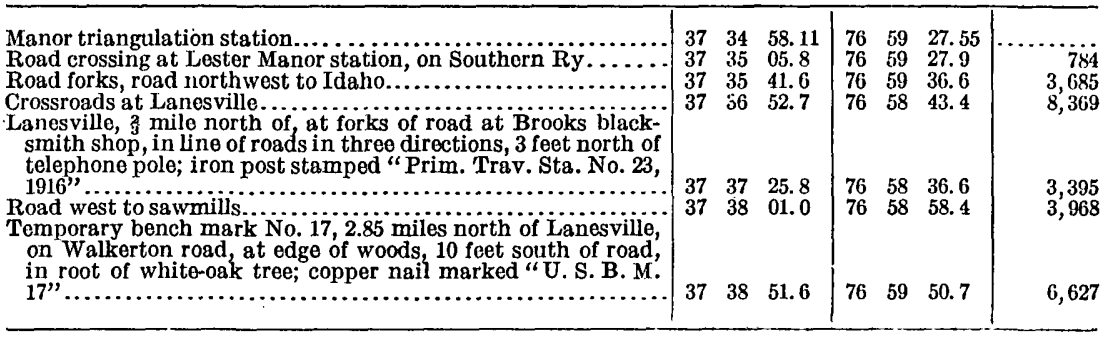

Magnetic declination near east border of quadrangle, $5^{\circ} 35^{\prime} \mathrm{W}$.; near west border of quadrangle, $5^{\circ} 35^{\prime} \mathrm{W}$. 
NEW KENT QUADRANGLE-Continued.

East along highways near north border of quadrangle.

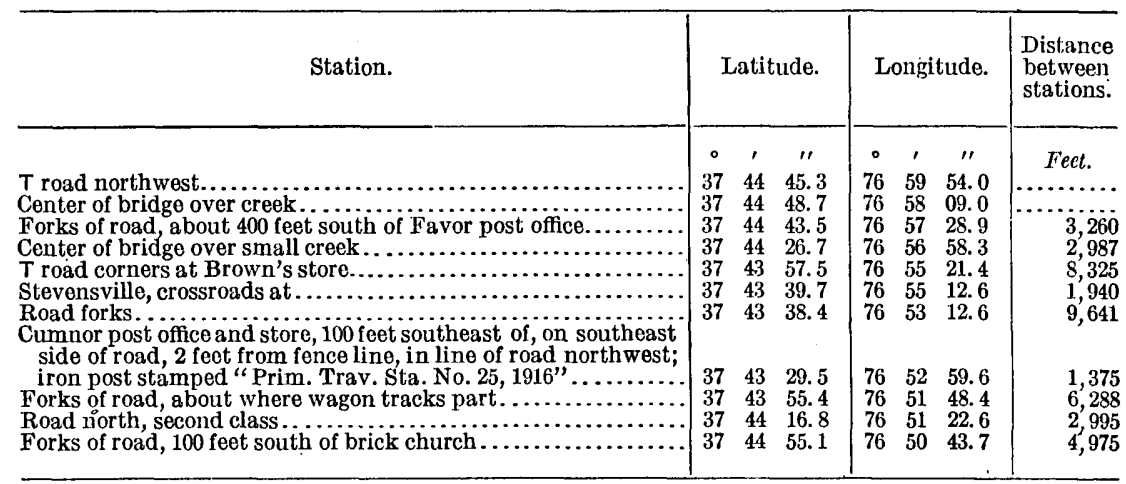

Magnetic declination for northwest quarter of quadrangle, $5^{\circ} 50^{\prime} \mathrm{W}$.

East along highways near south border of quadrangle.

Road forks, second-class road south.............................

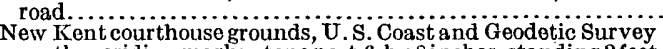

north meridian mark; stone post 6 by 8 inches, standing 2 feet

above ground, has been graded around; tower of courthouseis

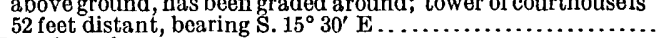

$T$ road north.

Crossroads.

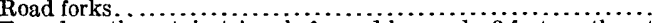

$T$ road southwest, in triangle formed by roads, 3 feet northeast

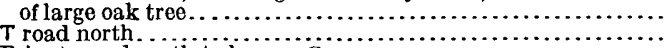

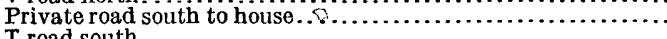

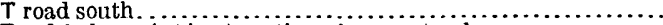

Road forks, point is at parting of wagon tracks...............

$T$ road south in woods, about 20 feet east of road north . . . . . . . . .

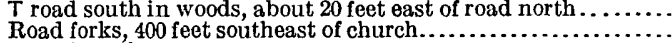

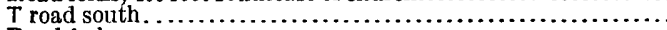

Road forks. .

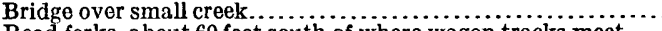

Road forks, about 60 feet south of where wagon tracks meet. .

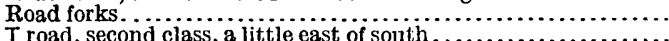

Brick House (2) triangulation station, on most promising point

of ground on west side of Y ork River southeast of West Point.

On each side of this point, which is 70 meters wide, a prong of marsh extends into the land. Station is 15 meters back from top of river bank and 30 meters from high-water line and about 8 feet above it, on center point of high ground of old field; cement block 8 by 8 by 24 inches with legend and triangle on brass cap cast in the top. Reference mark: Cement block with brass cap and arrow. Block is 8 by 8 by 24 inches and projects 1 inch above surface of ground, distant $50.203 \mathrm{me}$ -

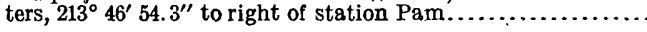

Magnetic declination along south border of quadrangle, $5^{\circ} 00^{\prime} \mathrm{W}$.

Along highways diagonally through the northeast quarter of quadrangle.

Creek, center of bridge over.

Road forks, at parting of wagon tracks

$T$ road forks, second-class road south.

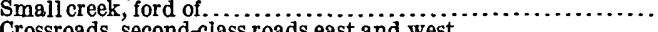

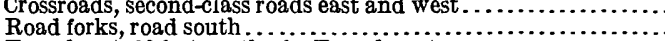

$T$ road east, 30 feet north of a $T$ road west.

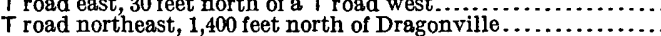

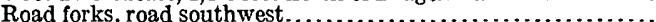

Road forks, road south.

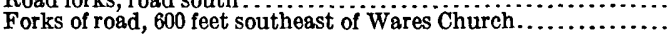

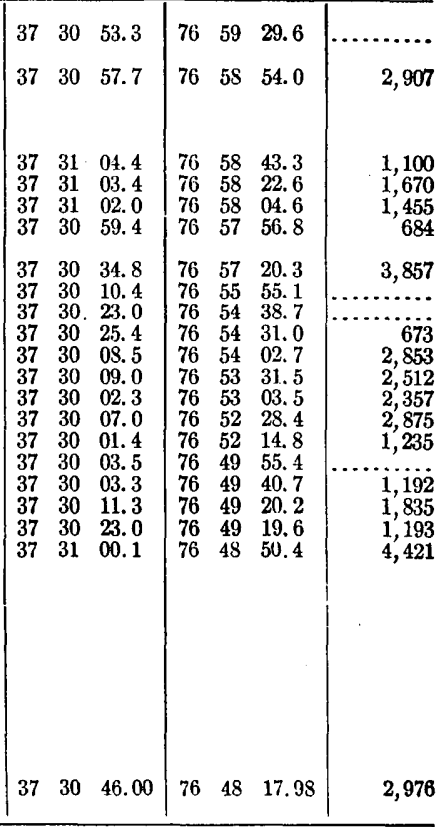

1,100

1,455

684

(673

512

357
2875

235

1,192

1,835
1,193

421

\begin{tabular}{|lll|lll|r}
37 & 44 & 24.4 & 76 & 47 & 46.1 & $\ldots \ldots \ldots$ \\
37 & 44 & 05.5 & 76 & 47 & 56.8 & 2,090 \\
37 & 43 & 46.5 & 76 & 47 & 29.5 & 2,918 \\
37 & 43 & 29.2 & 76 & 47 & 00.1 & 2,939 \\
37 & 43 & 13.9 & 76 & 46 & 51.2 & 1,703 \\
37 & 42 & 47.8 & 76 & 46 & 52.1 & 2,643 \\
37 & 42 & 31.3 & 76 & 47 & 03.4 & 1,893 \\
37 & 42 & 03.2 & 76 & 46 & 50.8 & $-3,015$ \\
37 & 41 & 30.1 & 76 & 46 & 29.4 & 3,769 \\
37 & 41 & 04.6 & 76 & 45 & 38.1 & 4,862 \\
37 & 40 & 49.14 & 76 & 45 & 04.52 & 3,127
\end{tabular}


NEW KENT QUADRANGLE-Con tinued.

West along highways through center of east half of quadrangle.

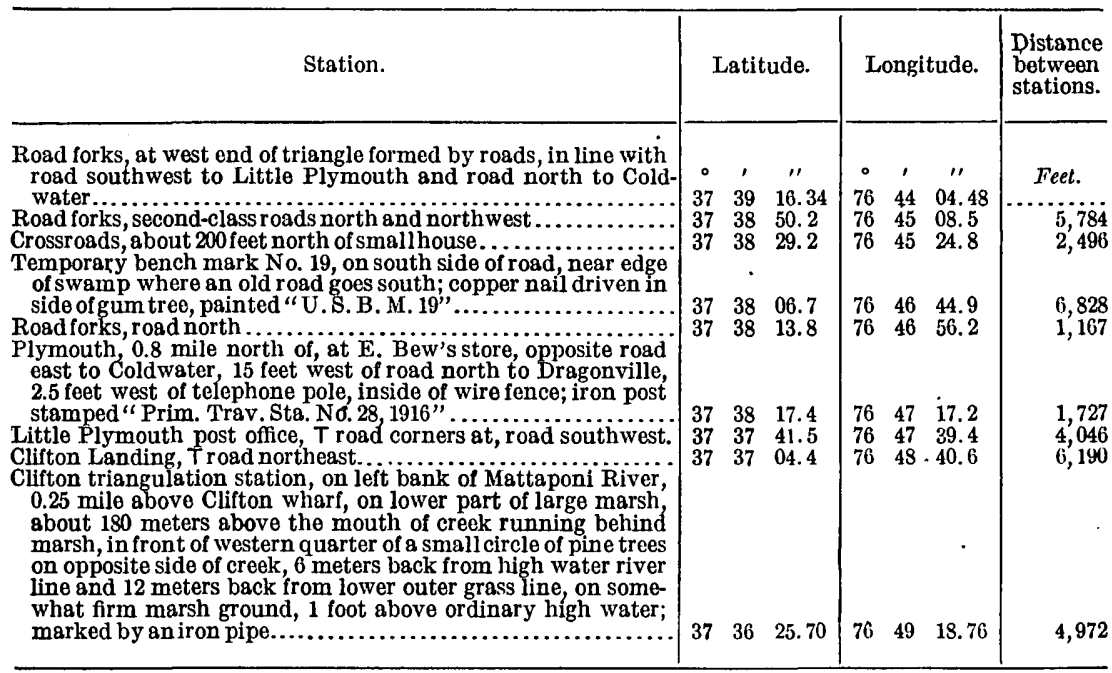

Magnetic declination for northeast quarter of quadrangle, $5^{\circ} 30^{\prime} \mathrm{W}$.; for center of east half of quadrangle, $5^{\circ} 30^{\prime} \mathrm{W}$.

NEWPORT NEWS QUADRANGLE.

South along Norfolk Southern R. R. (By F. J. McMaugh in 1917.)

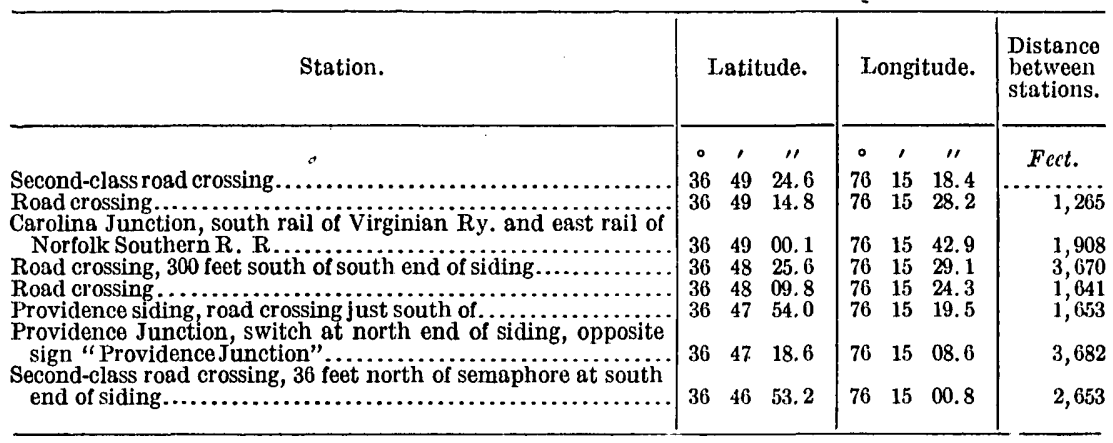

North along Virginian (Tidewater) Ry. (By F. J. McMaugh in 1917-18.)

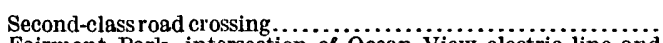

Fairmont Park, intersection of Ocean View electric line and

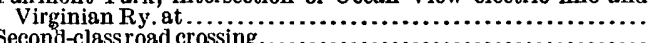

\begin{tabular}{rrr|rrr|r}
36 & 52 & 18.3 & 76 & 15 & 01.2 & $\ldots \ldots \ldots$ \\
36 & 52 & 36.8 & 76 & 15 & 05.7 & 1,892 \\
36 & 52 & 42.7 & 76 & 15 & 07.2 & 610 \\
36 & 53 & 05.5 & 76 & 15 & 12.9 & 2,354 \\
36 & 53 & 17.5 & 76 & 15 & 15.9 & 1,239 \\
36 & 53 & 50.9 & 76 & 15 & 24.2 & 3,447 \\
36 & 54 & 13.5 & 76 & 15 & 29.8 & 2,327 \\
36 & 54 & 27.5 & 76 & 15 & 35.9 & 1,503 \\
36 & 54 & 32.9 & 76 & 15 & 41.6 & 715 \\
36 & 55 & 00.0 & 76 & 16 & 21.3 & 4,234 \\
& & & & & \\
\hline
\end{tabular}

restle, 400 -foot, south end of, about 400 feet south of road cross-

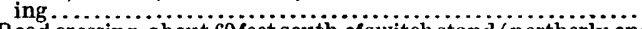
Road crossing, about 60 feet south of switch stand (northerly one

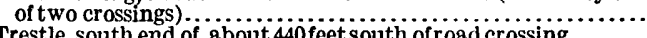

Sectie, south end of, about

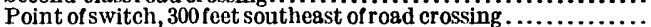

Intersection of Virginian $\mathbf{R y}$, and electric railroad, on north rai of Virginian and east rail of east track of electric road ..........

South along electric rallroad.

Navy Base road, center of, near A. C. Ward's corner, 25 feet north of concrete road, in line with east rail of electric road. 
NEWPORT NEWS QUADRANGLE-Continued.

West along highway.

\begin{tabular}{|c|c|c|c|}
\hline Station. & Latitude. & Longitude. & Distance \\
\hline 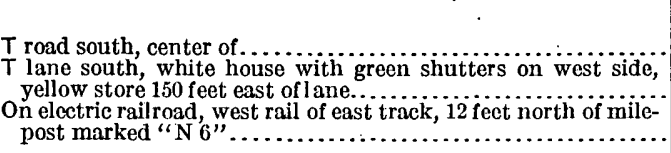 & $\begin{array}{ccc}\circ & \prime & \prime \prime \\
36 & 54 & 58.7 \\
36 & 54 & 57.7 \\
36 & 54 & 58.0\end{array}$ & $\begin{array}{ccc}0 & \prime & \\
76 & 16 & 59.0 \\
76 & 17 & 57.9 \\
76 & 18 & 31.9\end{array}$ & $\begin{array}{r}\text { Feet. } \\
3,002 \\
4,786 \\
2,758\end{array}$ \\
\hline
\end{tabular}

North along electric railroad.

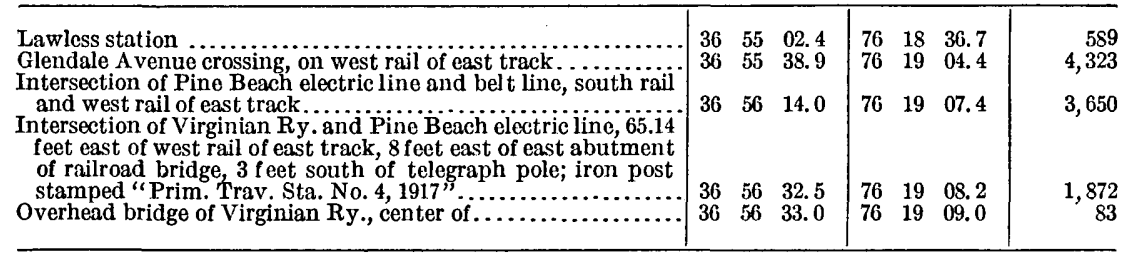

East along electric railroad from Pine Beach to Ocean View.

\begin{tabular}{|c|c|c|c|c|c|c|c|}
\hline Intersection of west rail of railroad spur and north rail of electric & & & \multirow[b]{2}{*}{40.0} & & & \multirow[b]{2}{*}{25.8} & \multirow[b]{2}{*}{3,582} \\
\hline $\begin{array}{l}\text { road } \\
\text { Pennsylvania State Building, tower of (U. S. Coast and Geo- }\end{array}$ & 36 & & & & & & \\
\hline detic Survey triangulation station)..$\ldots \ldots \ldots \ldots \ldots \ldots$ & 36 & 57 & 02.87 & 76 & 18 & 47. 44 & 2,899 \\
\hline Bush Creek, west end of bridge over..... & 36 & 56 & 38.3 & 76 & 18 & 0.7 .7 & 1,642 \\
\hline Road crossing . . . . . . . . & 36 & 56 & 27.0 & 76 & 17 & 33.8 & 2,837 \\
\hline Mason Creek, west end of bridge over. & 36 & 56 & 24.0 & 76 & 17 & 09.0 & 2,033 \\
\hline Mason Creek, center of draw span over. & 36 & 56 & 24.1 & 76 & 17 & 02.7 & 513 \\
\hline 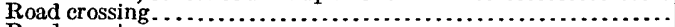 & 36 & 56 & 29.1 & 76 & 16 & 45.2 & 1,509 \\
\hline 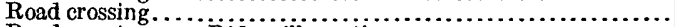 & 36 & 56 & 41. 0 & 76 & 16 & 27.4 & 1,880 \\
\hline Road crossing near Ridgevilie station... & 36 & 56 & 56.1 & 76 & 16 & 03.0 & 2,499 \\
\hline 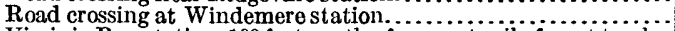 & 36 & 57 & 02.8 & 76 & 15 & 51.0 & 1,192 \\
\hline Virginia Bay station, 100 feet north of, on east rail of west track, & 36 & & & & & & \\
\hline Ocean View station, south end of.. & $\begin{array}{l}30 \\
36\end{array}$ & 57 & 12.4 & 70 & 15 & 02.6 & $\begin{array}{l}3,395 \\
2,304\end{array}$ \\
\hline
\end{tabular}

West on highway from Oxford station.

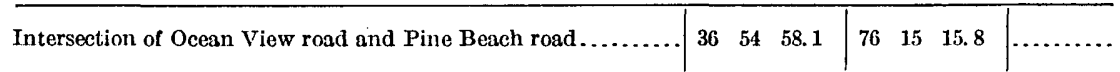

West along highways and railroads. (By F. J. McMaugh in 1918.)

Carolina Junction, intersection of Virginian $\mathrm{Ry}$. and Norfolk

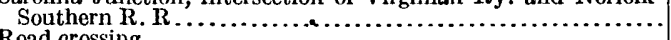

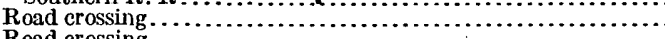

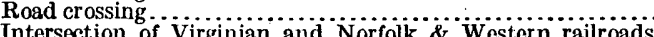
south and west rails. south and west rails $\ldots \ldots \ldots \ldots \ldots \ldots \ldots \ldots \ldots \ldots \ldots \ldots \ldots \ldots \ldots \ldots \ldots \ldots \ldots \ldots \ldots \ldots$
Middle of road, at trolley line $\ldots \ldots \ldots \ldots \ldots \ldots \ldots$

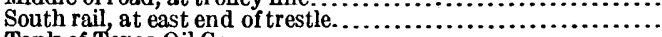

Tank of Texas Oil Co.

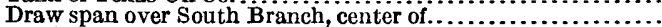

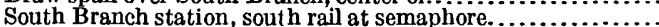

Intersection of Virginian $\mathrm{Ry}$. and trolley line.

Intersection of Deep Creek road and Virginian $\mathbf{R} y \ldots \ldots \ldots \ldots \ldots$

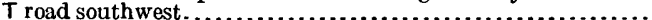

South end of bridge . . . . . . . . .

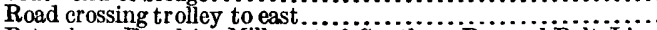

Petersburg Excelsior Mill, east of, Southern $\mathbf{R}$. and Beit $\mathbf{L}$ ine,

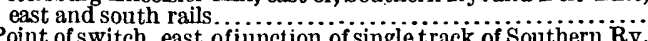

Point of switch, east of junction of single track of Southerm $\ddot{k}$

and main line of Seaboard Air Line Ry., cabin 100 feet south.

Avenue, 86 f eet north of, in southwest corner of S. $\dot{B}$. Carney's yard, 25 cet east of center line of road; iron post stamped

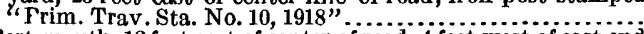

Port :mouth, 12 feet east of center of road, 4 feet west of east end of pipe culvert across Elm Avenue, near intersection of east and west road at Brick School; stake.

\begin{tabular}{|lll|lll|r}
36 & 49 & 00.1 & 76 & 15 & 42.9 & $\ldots \ldots$ \\
36 & 48 & 56.7 & 76 & 15 & 46.3 & \\
36 & 48 & 47.7 & 76 & 15 & 55.3 & 1,172 \\
36 & 48 & 34.2 & 76 & 16 & 08.9 & 1,756 \\
36 & 48 & 07.4 & 76 & 16 & 35.7 & 3,482 \\
36 & 47 & 48.4 & 76 & 16 & 59.5 & 2,721 \\
36 & 47 & 51.5 & 76 & 17 & 15.0 & 1,303 \\
36 & 47 & 49.4 & 76 & 17 & 35.3 & 1,666 \\
36 & 47 & 49.2 & 76 & 18 & 07.5 & 2,620 \\
36 & 47 & 44.1 & 76 & 18 & 41.9 & 2,817 \\
36 & 47 & 34.8 & 76 & 19 & 44.8 & 5,198 \\
36 & 48 & 13.8 & 76 & 19 & 20.0 & 4,429 \\
36 & 48 & 35.1 & 76 & 19 & 03.2 & 2,552 \\
36 & 49 & 01.8 & 76 & 19 & 31.4 & 3,544 \\
& & & & & & \\
36 & 49 & 26.4 & 76 & 19 & 47.2 & 2,800 \\
36 & 49 & 21.3 & 76 & 20 & 20.5 & 2,760 \\
& & & & & & \\
& & & & & & \\
36 & 49 & 11.1 & 76 & 20 & 42.8 & 2,084 \\
& & & & & & \\
36 & 49 & 44.4 & 76 & 20 & 51.4 & 3,442
\end{tabular}


NEWPORT NEWS QUADRANGLE-Continued.

West along highways and railroads-Continued.

\begin{tabular}{|c|c|c|c|}
\hline Station. & Latitude. & Longitude. & $\begin{array}{l}\text { Distance } \\
\text { between } \\
\text { stations. }\end{array}$ \\
\hline 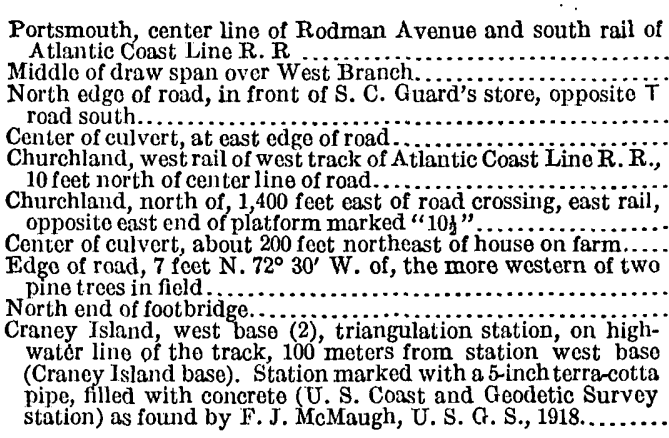 & $\begin{array}{lll}36 & 50 & 06.3 \\
36 & 50 & 31.6 \\
& & \\
36 & 51 & 03.5 \\
36 & 51 & 24.5 \\
& & \\
36 & 51 & 43.7 \\
& & \\
36 & 52 & 22.6 \\
36 & 52 & 48.5 \\
& & \\
36 & 53 & 01.1 \\
36 & 53 & 27.0 \\
& & \end{array}$ & \begin{tabular}{|ccc}
70 & 20 & 51.5 \\
76 & 21 & 42.6 \\
76 & 22 & 40.9 \\
76 & 23 & 10.1 \\
76 & 23 & 36.7 \\
76 & 23 & 45.1 \\
76 & 24 & 22.6 \\
76 & 24 & 07.2 \\
76 & 24 & 10.0
\end{tabular} & $\begin{array}{r}3,995 \\
4,021 \\
1,781 \\
2,634\end{array}$ \\
\hline
\end{tabular}

South along ralloads and highways. (By F. J. McMaugh in 1918.)

Churchland, west rail of west track of Atlantic Coast Line R. R.,

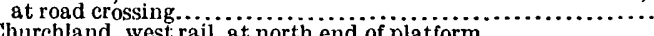

West rail, west rall, at trostlo

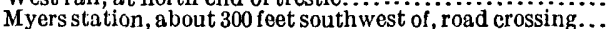

Public-road crossing, near north yard-limit sign; platform northeast of crossing, white house southeast..............................

north rail of Atlantic Coast Line $\mathbf{R}$.

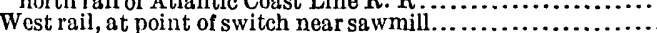

West rail, at public-road crossing, about 500 feet south of trestle; covered station platform southwest of crossing..................

Shoulder Hill, near northeast corner of freight platform, 23 feet west of west rail of railroad track; iron post stamped " Prim.

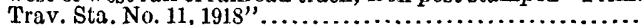

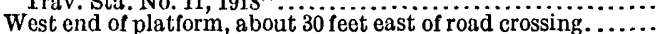

Peaks siding, south rail, at center of road

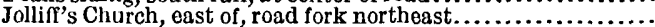

Jolliff's School, 67 feet $\mathrm{S} .63^{\circ} 31^{\prime} \mathrm{E}$. from front door of; iron post

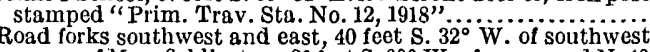
corner of Mansfield's store, 20 feet S. $60^{\circ}$ W. of pump and N. $40^{\circ}$ $\mathrm{W}$. of blazed pole on east side of road........................

Small T road north.

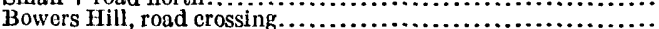

Center of bridge over creek....

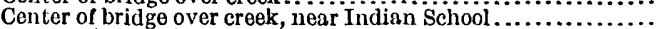

Intersection of highway and Virginian Ry., 93.6 feet northeast of; iron post stamped "Prim. Trav. Sta. No. 13, 1918"........

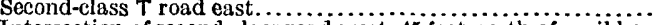

Intersection of second-class road west, 45 feet north of mail box

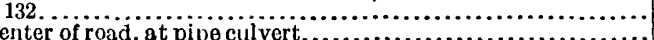

Center of road, at pipe culvert......................................... of three tracks.......................................

South rail of middle track opposite milepost $\mathrm{N} 10 \mathrm{~B} 398 . \ldots \ldots \ldots$

Yadkin station, 1 mile east of, at public-road crossing, 73 feet northeast of south rail of middle track; iron post stamped "Prim. Trav. Sta. No. 14, 1918"

South rail, opposite milepost N 9 B 399 , near point of switch, 60 feet east along track to road crossing......................

South rail of north track, at road crossing; elevation painted

"15.6"

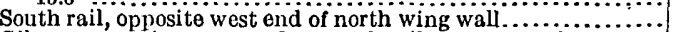

Gilmerton station, west end of, south rail of north track...........

West end of bridge approach, opposite operating house.........

Intersection of Elizabeth River Belt Lineand Norfolk \& Western

Ry., 76 feet southwest of; iron post stamped "Prim. Trav.

Sta. No. 15, 1918"

South rail of north track, at west end of treste, at road crossing.

Extreme west rail of most westerly track, at road crossing......

West rail, opposito milepost marked "N 4 B 404" ..............

South Norfolk Junction, intersection of Virginian Ry. and Nor-

folk \& Western Ry., south and west rails...................

\begin{tabular}{|c|c|c|c|c|c|}
\hline $\begin{array}{l}51 \\
51 \\
51 \\
51\end{array}$ & $\begin{array}{l}43.7 \\
44.8 \\
31.8 \\
08.4\end{array}$ & $\begin{array}{l}76 \\
76 \\
76 \\
76\end{array}$ & $\begin{array}{l}23 \\
23 \\
24 \\
24\end{array}$ & $\begin{array}{l}36.7 \\
45.1 \\
14.3 \\
57.8\end{array}$ & $\begin{array}{r}693 \\
2,717 \\
4,252\end{array}$ \\
\hline 50 & 46.2 & 76 & 25 & 38.8 & 4,016 \\
\hline $\begin{array}{l}50 \\
50\end{array}$ & $\begin{array}{l}33.3 \\
01.4\end{array}$ & $\begin{array}{l}76 \\
76\end{array}$ & $\begin{array}{l}26 \\
27\end{array}$ & $\begin{array}{l}02.6 \\
01.2\end{array}$ & $\begin{array}{l}2,334 \\
5,762\end{array}$ \\
\hline 49 & 32.5 & 76 & 27 & 54.0 & 5,188 \\
\hline $\begin{array}{l}49 \\
48 \\
48 \\
48\end{array}$ & $\begin{array}{l}09.0 \\
50.5 \\
55.5 \\
38.8\end{array}$ & $\begin{array}{l}76 \\
76 \\
76 \\
76\end{array}$ & $\begin{array}{l}28 \\
27 \\
26 \\
26\end{array}$ & $\begin{array}{l}37.3 \\
09.7 \\
34.0 \\
09.8\end{array}$ & $\begin{array}{l}4,248 \\
7,360 \\
2,948 \\
2,591\end{array}$ \\
\hline 48 & 07.8 & 76 & 20 & 22.7 & 3,310 \\
\hline $\begin{array}{l}47 \\
47 \\
47 \\
47 \\
46\end{array}$ & $\begin{array}{l}48.3 \\
21.8 \\
10.0 \\
00.8 \\
59.0\end{array}$ & $\begin{array}{l}76 \\
76 \\
76 \\
76 \\
76\end{array}$ & $\begin{array}{l}20 \\
25 \\
24 \\
24 \\
24\end{array}$ & $\begin{array}{l}04.0 \\
16.0 \\
45.3 \\
34.2 \\
03.1\end{array}$ & $\begin{array}{l}2,486 \\
4,732 \\
2,775 \\
1,289 \\
2,540\end{array}$ \\
\hline $\begin{array}{l}46 \\
46\end{array}$ & $\begin{array}{l}56.8 \\
35.0\end{array}$ & $\begin{array}{l}76 \\
76\end{array}$ & $\begin{array}{l}23 \\
23\end{array}$ & $\begin{array}{l}56.4 \\
23.8\end{array}$ & $\begin{array}{r}588 \\
3,449\end{array}$ \\
\hline $\begin{array}{l}46 \\
46\end{array}$ & $\begin{array}{l}22.4 \\
03.2\end{array}$ & $\begin{array}{l}76 \\
76\end{array}$ & $\begin{array}{l}22 \\
21\end{array}$ & $\begin{array}{l}32.6 \\
48.8\end{array}$ & $\begin{array}{l}4,358 \\
4,060\end{array}$ \\
\hline $\begin{array}{l}45 \\
45\end{array}$ & $\begin{array}{l}49.7 \\
54.8\end{array}$ & $\begin{array}{l}76 \\
76\end{array}$ & $\begin{array}{l}21 \\
21\end{array}$ & $\begin{array}{l}43.8 \\
13.0\end{array}$ & $\begin{array}{l}1,421 \\
2,553\end{array}$ \\
\hline 40 & 00.8 & 76 & 20 & 40.3 & 2,729 \\
\hline 46 & 05.5 & 76 & 20 & 08.6 & 2,619 \\
\hline $\begin{array}{l}46 \\
46 \\
46 \\
46\end{array}$ & $\begin{array}{l}12.4 \\
16.1 \\
21.5 \\
29.4\end{array}$ & $\begin{array}{l}76 \\
76 \\
76 \\
76\end{array}$ & $\begin{array}{l}19 \\
19 \\
18 \\
17\end{array}$ & $\begin{array}{l}27.4 \\
05.0 \\
32.8 \\
45.1\end{array}$ & $\begin{array}{l}3,425 \\
1,865 \\
2,672 \\
3,964\end{array}$ \\
\hline $\begin{array}{l}46 \\
46 \\
47 \\
48\end{array}$ & $\begin{array}{l}32.7 \\
43.5 \\
07.3 \\
01.9\end{array}$ & $\begin{array}{l}76 \\
76 \\
76 \\
76\end{array}$ & $\begin{array}{l}17 \\
16 \\
16 \\
10\end{array}$ & $\begin{array}{l}21.9 \\
47.6 \\
17.6 \\
02.9\end{array}$ & $\begin{array}{l}1,912 \\
3,001 \\
3,428 \\
5,944\end{array}$ \\
\hline 48 & 34.2 & 76 & 10 & 08.9 & 2,998 \\
\hline
\end{tabular}

Magnetic declination for quadrangle, $5.0^{\circ} \mathrm{W}$. 
NEWPORT NEWS QUADRANGLE-Continued.

Along Southern Ry. In southwest part of quadrangle. (By H. J. Switzer in 1917-18.)

\begin{tabular}{|c|c|c|c|}
\hline Station. & Latitude. & Longitude. & $\begin{array}{l}\text { Distance } \\
\text { between } \\
\text { stations. }\end{array}$ \\
\hline 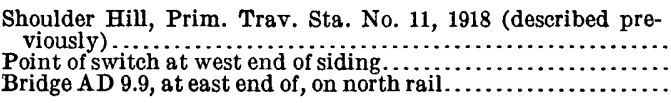 & $\begin{array}{lcl}\circ & \prime & \prime \prime \\
36 & 49 & 09.02 \\
36 & 48 & 50.0 \\
36 & 48 & 36.4\end{array}$ & $\begin{array}{lll}0 & \prime & \prime \prime \\
76 & 28 & 37.28 \\
76 & 29 & 11.4 \\
76 & 29 & 36.1\end{array}$ & $\begin{array}{l}\text { Feet. } \\
3,381 \\
2,434\end{array}$ \\
\hline
\end{tabular}

Northeast along highways. (By J. J. Charters in 1918.)

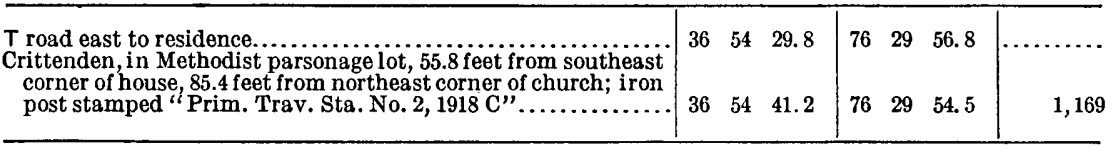

\section{PETERSBURG QUADRANGLE.}

South along highways near east border of quadrangle. (By J. H. Wilson in 1916-17.)

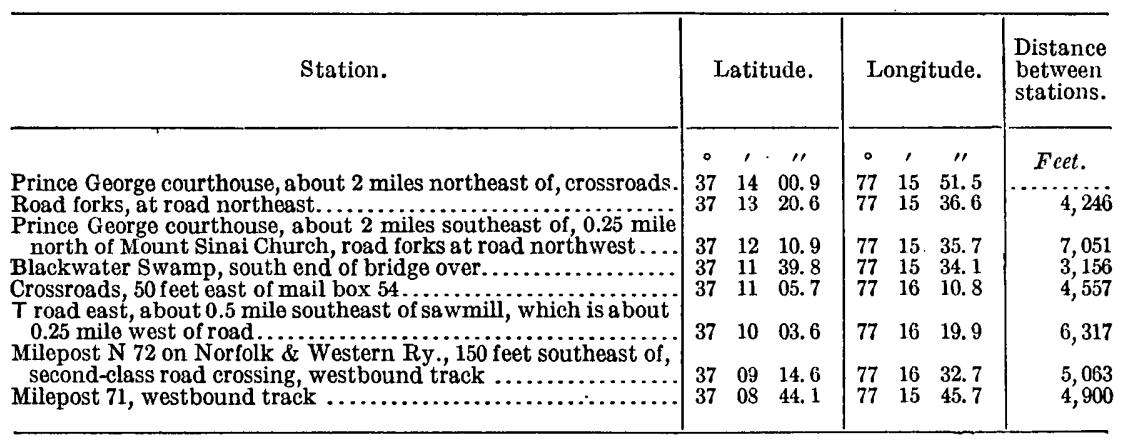

Along highways in southeast corner of quadrangle.

Jerusalem plank road, at road northeast to Disputanta, just

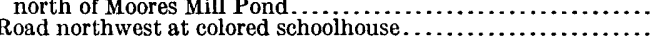

Bridge over creek.

\begin{tabular}{lll|lll|l}
37 & 00 & 43.7 & 77 & 16 & 32.0 & $\ldots \ldots \ldots$ \\
37 & 01 & 48.4 & 77 & 15 & 30.3 & \\
37 & 02 & 39.4 & 77 & 15 & 02.6 & \\
& & & 5,6256
\end{tabular}

Magnetic declination along east border of quadrangle, $3^{\circ} 39^{\prime} \mathrm{W}$; for southeast corner of quadrangle, $2^{\circ} 30^{\prime} \mathrm{W}$.

On highway near southwest corner of quadrangle. (By J. C. Fales in 1918.)

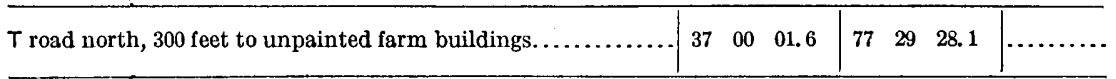

PINEY POINT QUADRANGLE.

West along highways near south border of quadrangle.

\begin{tabular}{|c|c|c|c|c|c|c|c|}
\hline \multirow[t]{2}{*}{ Station. } & \multicolumn{3}{|c|}{ Latitude. } & \multicolumn{3}{|c|}{ Longitude. } & \multirow{2}{*}{$\begin{array}{c}\begin{array}{c}\text { Distance } \\
\text { between } \\
\text { stations. }\end{array} \\
\text { Feet. }\end{array}$} \\
\hline & & , & ", & & 1 & & \\
\hline T road corners $\ldots \ldots \ldots \ldots \ldots \ldots \ldots \ldots \ldots \ldots \ldots$ & 38 & 00 & 00.1 & 76 & 34 & 57.8 & $(4,129)$ \\
\hline North end of a 350 -foot pile bridge over inlet................... & 38 & 00 & 27.1 & 76 & 35 & 11.6 & $\quad 2,952$ \\
\hline $\begin{array}{l}T \text { road corners, road northeast to Kensale.......................... } \\
\text { Old Bear Corners, forks of road at, } 80 \text { feet southwest of front of }\end{array}$ & 38 & 01 & 07.2 & 76 & 36 & 11.0 & 6,245 \\
\hline Mrs. Evan's store, about in line with road westerly and road & & & & & & & \\
\hline southeast; iron post stamped "Prim. Trav. Sta. No. 15, 1916" & 38 & 01 & 14. 2 & 76 & 36 & 29.7 & 1,653 \\
\hline 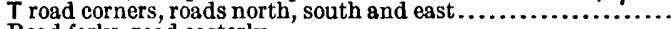 & 38 & 01 & 42.4 & 76 & 37 & 52.9 & 7,245 \\
\hline $\begin{array}{l}\text { Road forks, road easterly } \\
\text { Three corners at Acorn, about in line with roads in three direc- }\end{array}$ & 38 & 01 & 17.9 & 76 & 38 & 21.7 & 3,387 \\
\hline 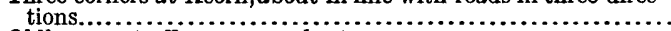 & 38 & 01 & 09.9 & 76 & 38 & 56.9 & 2,930 \\
\hline Oldham post office, crossroads at $\ldots \ldots \ldots \ldots \ldots \ldots \ldots \ldots \ldots \ldots \ldots \ldots \ldots \ldots \ldots$ & 38 & 00 & 23.1 & 76 & 39 & 49.5 & 6,327 \\
\hline
\end{tabular}


PINEY POINT QUADRANGLE-Continued.

Along highways in southwest corner of quadrangle.

\begin{tabular}{|c|c|c|c|}
\hline Station. & Latitude. & Longitude. & $\begin{array}{l}\text { Distance } \\
\text { between } \\
\text { stations. }\end{array}$ \\
\hline 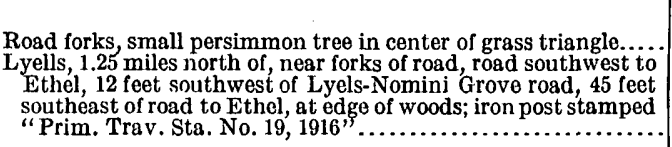 & $\begin{array}{ccc}\circ & \prime & \prime \prime \\
38 & 00 & 23.4 \\
38 & 00 & 41.6\end{array}$ & $\begin{array}{ccc}\circ & 1 & \prime \prime \\
76 & 43 & 42.7 \\
& & \\
76 & 44 & 11.8\end{array}$ & $\begin{array}{l}\text { Feet. } \\
(3,349)\end{array}$ \\
\hline
\end{tabular}

Magnetic declination near south border of quadrangle, $5^{\circ} 45^{\prime} \mathrm{W}$.

PORT ROYAL QUADRANGLE.

Along highways in southeast corner of quadrangle.

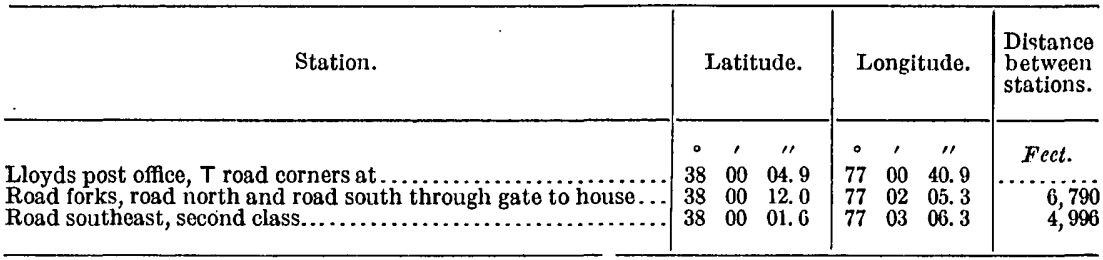

Magnetic declination for southeast corner of quadrangle, $4^{\circ} 50^{\prime} \mathrm{W}$.

\section{RICHMOND QUADRANGLE.}

East along highways near north border of quadrangle. (By E. L. McNair in 1916.)

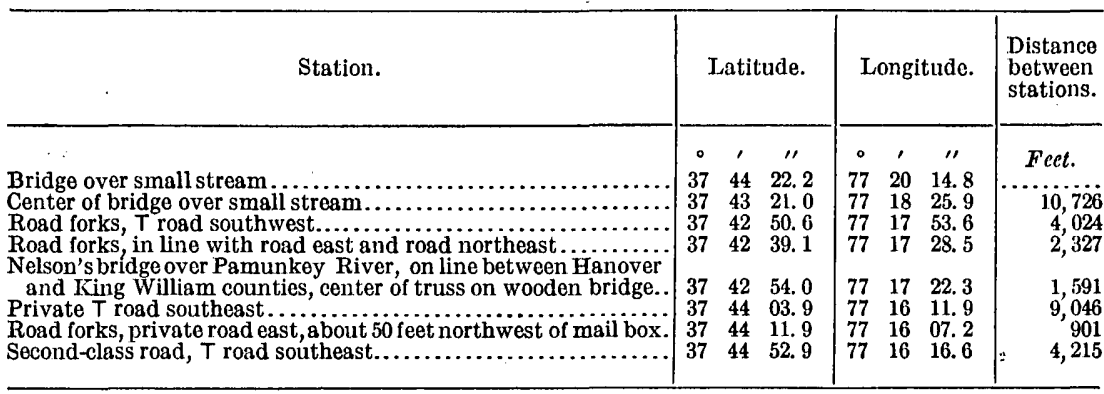

Magnetic declination for north border of quadrangle, $5^{\circ} 05^{\prime} \mathrm{W}$.

Along highways through northeast corner of quadrangle.

NoTE.-The positions on this line have not been adjusted, as the closing error of $3.24^{\prime \prime}$ in longitude is excessive. It is thought that a mistake in measurement of 300 feet may have been made between the position of station 4219 , at angle in road, and that of station 4221, at $T$ road south, on the King William sheet. The positions as given for this sheet are based on the adjusted position of station 2981 at road forks.

Road forks, road southwest.

Road forks, road southwest.

$\begin{array}{lll}37 & 42 & 39.1 \\ 37 & 42 & 26.7\end{array}$

$\begin{array}{lll}37 & 42 & 26.7 \\ 37 & 41 & 58.4\end{array}$

$\begin{array}{lll}37 & 41 & 58.4\end{array}$

$\begin{array}{lll}37 & 41 & 57.8\end{array}$ 
SMITHFIELD QUADRANGLE.

South along highways through center of quadrangle. (By J. J. Charters in 1918.)

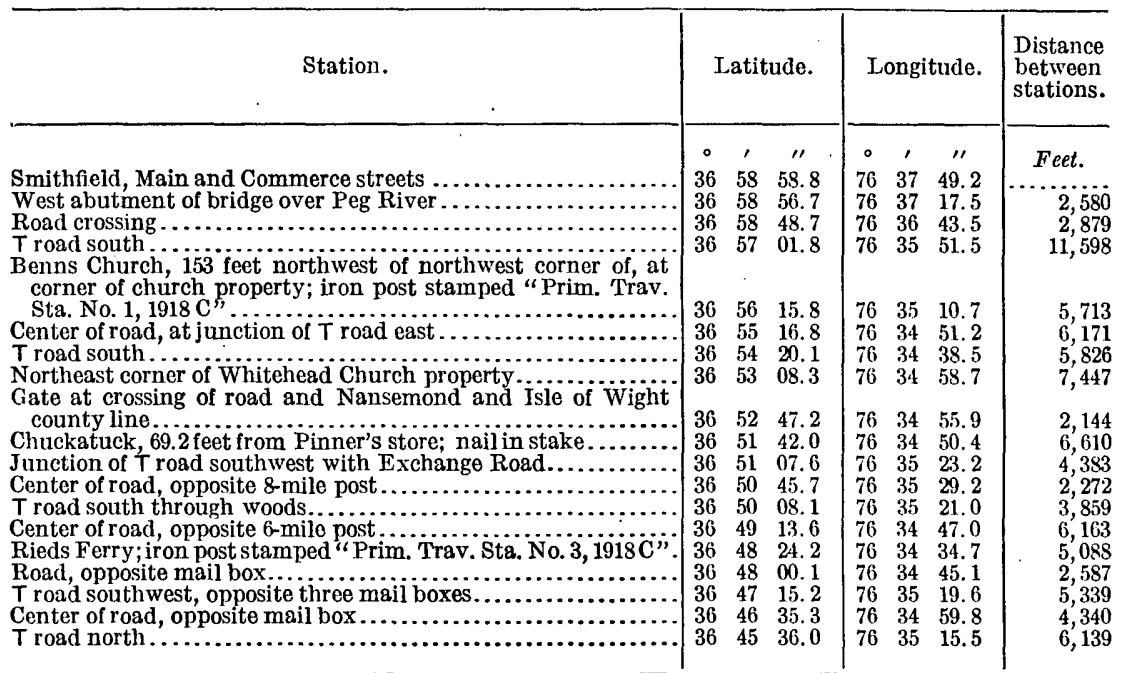

From Chuckatuck northeast along highways. (Checked spur line.)

Chuckatuck, at store, nail in stake.

$T$ road east.

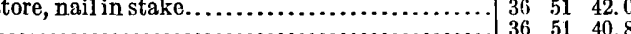

Troad north ....

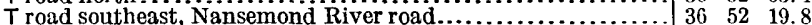

Center of road, opposite mail box of T. L. Griffin ..............

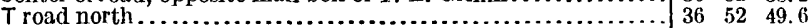

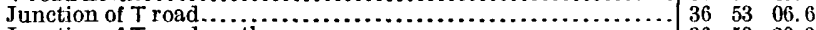

Junction of T road north

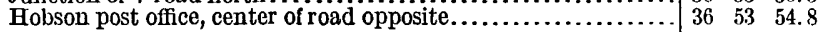

\begin{tabular}{lll|r}
76 & 34 & 50.4 & $\ldots \ldots \ldots$ \\
76 & 34 & 04.8 & 3,709 \\
76 & 33 & 26.8 & 3,845 \\
76 & 33 & 03.2 & 2,537 \\
76 & 32 & 18.6 & 4,074 \\
76 & 32 & 07.2 & 1,478 \\
76 & 31 & 14.0 & 4,650 \\
76 & 31 & 05.4 & 2,495 \\
76 & 30 & 34.4 & 3,540
\end{tabular}

Southwest along highways from Chuckatuck to Windsor.

Chuckatuck, at store, nail in stake.

Junction of Exchange-Suffolk road...

T road west, opposite two mail boxes.

.

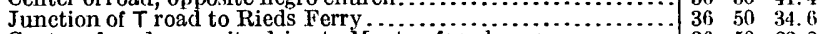

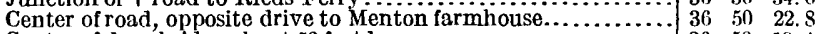

Center of drawbridge about 50 feet long..................... $36 \quad 50 \quad 19.4$

Everett, 38.5 feet from northeast corner of Saunder's house, in

northeast corner of yard; iron post stamped "Prim. Trav.

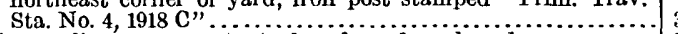

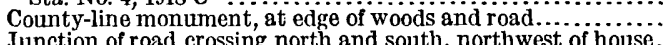

$T$ road north, at 'Griffith's mail box..........................

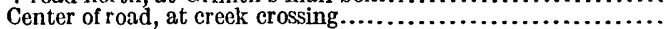

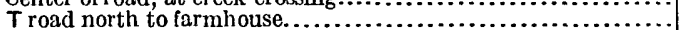

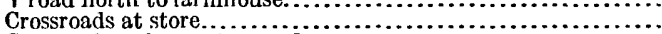

Center of creek crossing road.

Wooden bridge over creek.

T road north at Holland's mail box

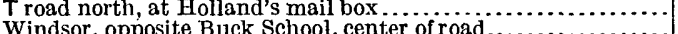

Windsor, opposite Buck School, center of road..............

tablet stamped "Prim. Trav. Sta. No. 8, 1918 C" ............

$36 \quad 50 \quad 18.4$

$50 \quad 07.2$

$\begin{array}{lll}49 & 48.7\end{array}$

4933.3

$49 \quad 18.6$

$49 \quad 15.4$

$\begin{array}{ll}49 & 12.9\end{array}$

$48 \quad 52.6$

$\begin{array}{ll}48 & 40.2\end{array}$

$\begin{array}{lll}48 & 37.0\end{array}$

$\begin{array}{lll}36 & 48 & 32.2\end{array}$

$\begin{array}{lll}36 & 48 & 28.9\end{array}$

\begin{tabular}{lll|r}
76 & 34 & 50.4 & $\ldots \ldots$ \\
76 & 35 & 23.6 & 4,419 \\
76 & 35 & 40.1 & 1,910 \\
76 & 35 & 54.5 & 1,712 \\
76 & 36 & 11.6 & 1,547 \\
76 & 37 & 10.4 & 4,917 \\
76 & 37 & 35.4 & 2,061 \\
& & & \\
76 & 37 & 43.0 & \\
76 & 38 & 27.5 & 621 \\
76 & 38 & 56.1 & 3,787 \\
76 & 39 & 29.3 & 3,015 \\
76 & 40 & 08.1 & 3,090 \\
76 & 40 & 43.6 & 3,480 \\
76 & 41 & 01.8 & 2,900 \\
76 & 41 & 37.1 & 1,498 \\
76 & 42 & 48.1 & 3,526 \\
76 & 43 & 35.2 & 5,907 \\
76 & 44 & 29.4 & 3,838 \\
& & & 4,431 \\
76 & 44 & 42.5 & 1,114
\end{tabular}

Northwest along Norfolk \& Western Ry, and highways.

Road crossing

Myrtle, road crossing at

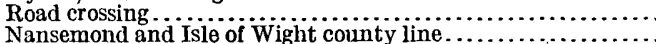

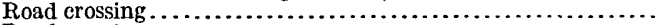

Road crossing

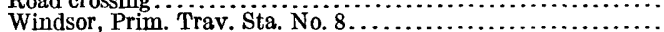

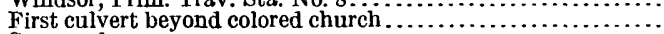

Crossroads.

$\begin{array}{lll}36 & 45 & 35.5 \\ 36 & 46 & 22.7 \\ 36 & 46 & 56.5 \\ 36 & 47 & 01.0 \\ 36 & 47 & 27.5 \\ 36 & 48 & 03.6 \\ 36 & 48 & 28.9 \\ 36 & 48 & 59.8 \\ 36 & 50 & 16.9\end{array}$

\begin{tabular}{rrr|r}
76 & 40 & 20.0 & $\ldots \ldots$ \\
76 & 41 & 31.4 & 7,520 \\
76 & 42 & 22.9 & 5,400 \\
76 & 42 & 29.7 & 721 \\
76 & 43 & 09.8 & 4,219 \\
76 & 44 & 04.5 & 5,750 \\
76 & 44 & 42.5 & 4,015 \\
76 & 44 & 42.8 & 3,127 \\
76 & 44 & 50.9 & 7,824
\end{tabular}


SMITHFIELD QUADRANGI,E-Continued.

Northeast along highways near west border of quadrangle.

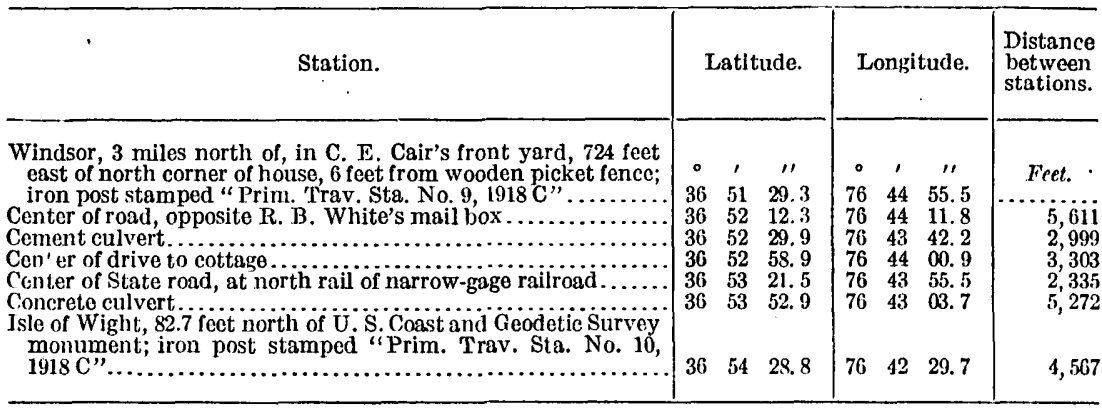

\section{Southwest along highways.}

Benns Church, Prim. Trav. Sta. No.1.

Junction of Isle of Wight and Smithfield roads

$T$ road north, center line south of both roads

Center line of road to Isle of Wight.

$T$ road south, center line south of both roads

Crossroads.

T road opposite mail box

Crossroads at Hart's store.

East gatepost of Allmond's farm

Junction of Bowling Green road

Isle of Wight, Prim. Trav. Sta. No. 10

\begin{tabular}{|lll|lll|r}
36 & 56 & 15.8 & 76 & 35 & 10.7 & $\ldots \ldots$. \\
36 & 56 & 16.3 & 76 & 30 & 20.7 & $\mathbf{5}, \mathbf{6 8 3}$ \\
36 & 55 & 35.1 & 76 & 37 & 10.5 & 5,809 \\
36 & 55 & 01.8 & 76 & 37 & 28.4 & 3,678 \\
36 & 54 & 36.2 & 76 & 38 & 09.2 & 4,204 \\
36 & 54 & 18.2 & 76 & 38 & 44.0 & 3,369 \\
36 & 54 & 06.5 & 76 & 39 & 33.9 & 4,224 \\
36 & 54 & 00.1 & 76 & 40 & 32.3 & 4.793 \\
36 & 54 & 28.8 & 76 & 40 & 56.5 & 3,508 \\
36 & 54 & 40.6 & 76 & 42 & 13.0 & 6,330 \\
36 & 54 & 28.8 & 76 & 42 & 29.7 & 1,809 \\
\end{tabular}

Magnetic declination for center of quadrangle, $5.8^{\circ} \mathrm{W}$.; for northeast part of quadrangle, $6.3^{\circ} \mathrm{W}$.; for south part of quadrangle, $5.5^{\circ} \mathrm{W}$.; for west part of quadrangle $5.6^{\circ} \mathrm{W}$; for southwest part of quadrangle, $5.5^{\circ} \mathrm{W}$.

Southwest along Southern Ry. (By H. J. Switzer in 1917-18.)

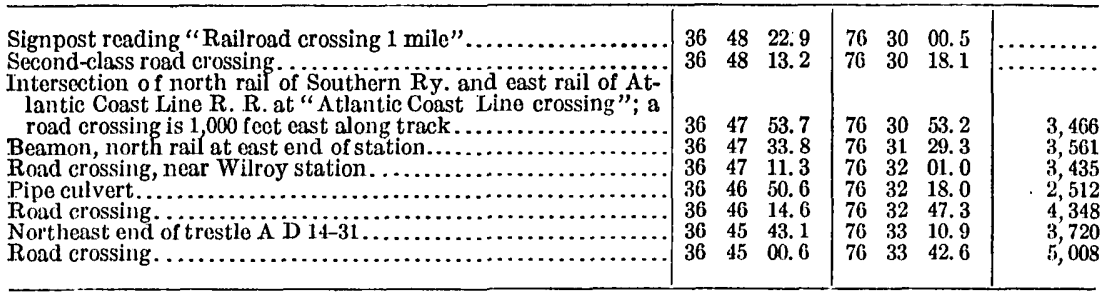

Northwest along highways in northwest corner of quadrangle. (By J. J. Charters in 1918.)

\begin{tabular}{|c|c|c|c|c|c|c|c|}
\hline Concrete culvert (located).... & 36 & 53 & 52.9 & 76 & 43 & 03.7 & \\
\hline 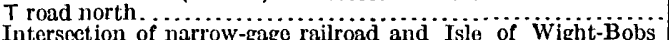 & 36 & 54 & 11.7 & 76 & 43 & 17.3 & \\
\hline 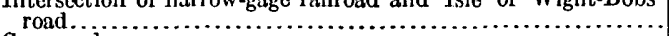 & 36 & 54 & 41.0 & 76 & 44 & 30.6 & 6,646 \\
\hline 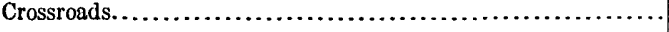 & 33 & 54 & 47.9 & 76 & 44 & 50.9 & 1,790 \\
\hline
\end{tabular}

South along highways near west border of quadrangle.

Windsor, Prim. Trav. Sta. No. 8 (previously Jocated). Center line of road, at first turn out of Windsor on road to Boaz. Center line of road, opposite C. F. Harris's mail box............ Junction of double culvert and road in woods.................. Troad southeast.

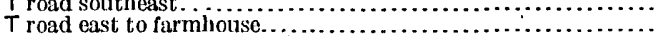
Center of north end of small wooden bridge.

Junction of crosstoads bearing east and west and north and

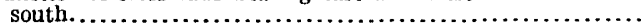

\begin{tabular}{|rrr|rrr|r}
36 & 48 & 28.9 & 76 & 44 & 42.5 & $\ldots \ldots, \ldots$ \\
36 & 48 & 15.9 & 76 & 44 & 48.2 & $\mathbf{1}, 388$ \\
36 & 47 & 47.2 & 76 & 44 & 48.7 & 2,892 \\
36 & 47 & 37.0 & 76 & 44 & 41.5 & 1,182 \\
36 & 46 & 52.4 & 76 & 44 & 45.9 & 4,512 \\
36 & 46 & 30.8 & 76 & 44 & 43.9 & 2,189 \\
36 & 45 & 24.8 & 76 & 44 & 57.6 & $\ldots \ldots \ldots$ \\
& & & & & \\
36 & 45 & 06.0 & 76 & 44 & 52.2 & 1,939 \\
\hline
\end{tabular}


SOUTH HILL QUADRANGLE.

Southwest along Southern Ry. through northeast quarter of quadrangle. (By J. C. Fales in 1918.)

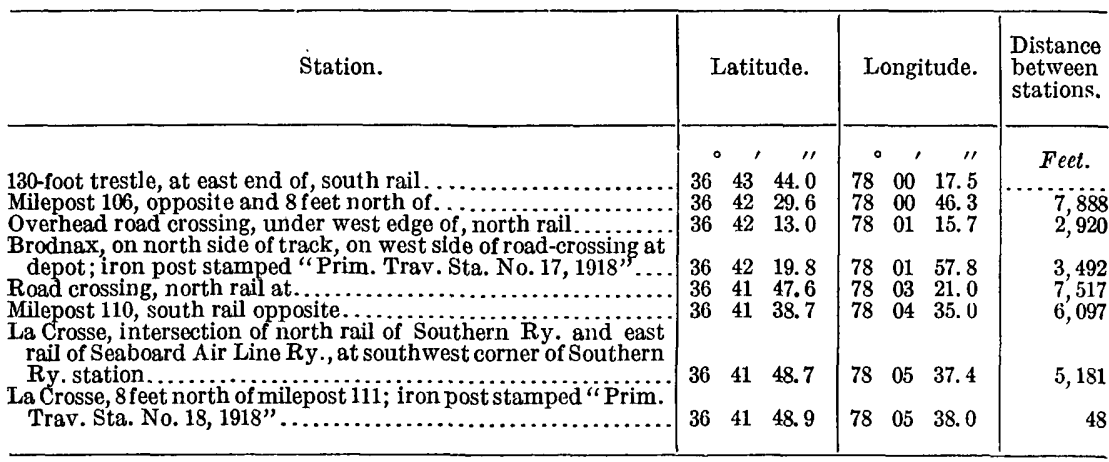

Southwest along Seaboard Air Line Ry. through northeast quarter of quadrangle.

\begin{tabular}{|c|c|c|c|c|c|c|c|}
\hline 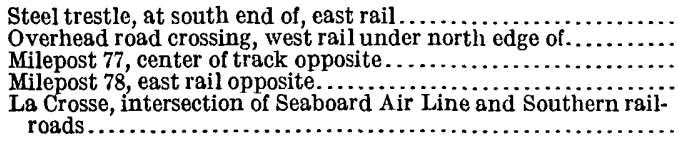 & $\begin{array}{l}36 \\
36 \\
36 \\
36 \\
36\end{array}$ & $\begin{array}{l}44 \\
43 \\
43 \\
42 \\
41\end{array}$ & $\begin{array}{l}42.8 \\
43.5 \\
17.6 \\
33.5 \\
48.7\end{array}$ & $\begin{array}{l}78 \\
78 \\
78 \\
78 \\
78\end{array}$ & $\begin{array}{l}03 \\
04 \\
04 \\
05 \\
05\end{array}$ & $\begin{array}{l}35.1 \\
23.0 \\
51.2 \\
22.7 \\
\\
37.4\end{array}$ & $\begin{array}{r}7,155 \\
3,482 \\
5,142 \\
4,684\end{array}$ \\
\hline
\end{tabular}

Along highways. (By J. C. Fales in 1918.)

Crichton post office, north edge of road, opposite front door of

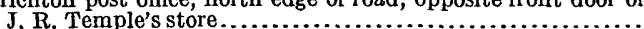
T road to Brodnax. . . . . . . . . .

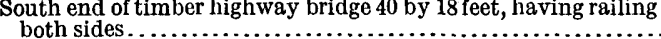

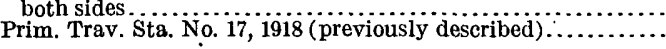

\begin{tabular}{|lll|lll|r}
36 & 40 & 17.0 & 78 & 00 & 47.2 & $\ldots \ldots \ldots$ \\
36 & 40 & 29.5 & 78 & 01 & 31.1 & 3,799 \\
36 & 41 & 09.6 & 78 & 01 & 49.2 & 4,314 \\
36 & 42 & 19.81 & 78 & 01 & 57.77 & 7,130 \\
\hline
\end{tabular}

\section{SUFFOLK QUADRANGLE.}

South on highways in northeast part of quadrangle. (By J. J. Charters in 1918.)

\begin{tabular}{|c|c|c|c|}
\hline Station. & Latitude. & Longitude. & Distance \\
\hline 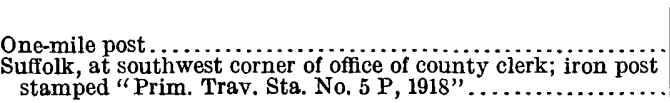 & $\begin{array}{ccc}\circ & \prime & \prime \prime \\
36 & 44 & 53.4 \\
36 & 44 & 16.5\end{array}$ & $\begin{array}{ccc}\circ & \prime & \prime \prime \\
76 & 35 & 01.2 \\
76 & 34 & 58.4\end{array}$ & $\begin{array}{r}\text { Feet. } \\
3,740\end{array}$ \\
\hline
\end{tabular}

West on Virginian Ry. near north border of quadrangle.

Road crossing

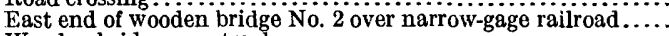

Wooden bridge over tracks

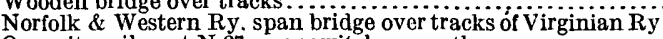

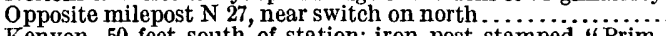

Kenyon, 50 feet south of station; iron post stamped "Prim.

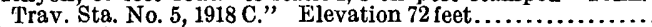

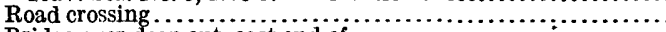

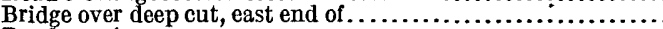

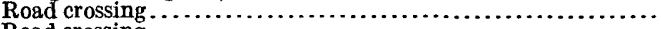

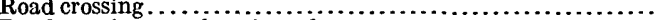

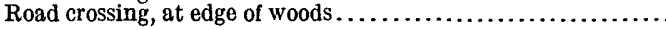

\begin{tabular}{|lll|lll|l}
\hline 36 & 44 & 03.1 & 76 & 35 & 47.2 & 4,198 \\
36 & 44 & 00.1 & 76 & 36 & 22.8 & 2,913 \\
36 & 43 & 52.6 & 76 & 37 & 27.6 & $\mathbf{5}, 330$ \\
36 & 43 & 51.1 & 76 & 37 & 41.6 & $\mathbf{1}, 144$ \\
36 & 43 & 54.9 & 76 & 38 & 37.4 & 4,568 \\
& & & & & & \\
36 & 43 & 59.8 & 76 & 39 & 20.4 & 3,536 \\
36 & 44 & 03.4 & 76 & 39 & 45.3 & 2,058 \\
36 & 44 & 13.5 & 76 & 41 & 05.1 & 6,580 \\
36 & 44 & 26.2 & 76 & 42 & 46.0 & $\mathbf{8}, 318$ \\
36 & 44 & 35.6 & 76 & 44 & 00.2 & $\mathbf{6}, 122$ \\
36 & 44 & 41.7 & 76 & 44 & 48.6 & 3,990 \\
& & & & & & \\
\hline
\end{tabular}

Northwest on Norfolk \& Western Ry, near north border of quadrangle.

Span bridge over tracks of Virginian Ry

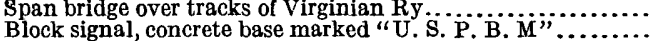

Road crossing, King road.

\begin{tabular}{|lll|lll|r}
36 & 43 & 51.1 & 76 & 37 & 41.6 & $\ldots \ldots$ \\
36 & 44 & 25.2 & 76 & 38 & 33.4 & 5,454 \\
36 & 44 & 54.4 & 76 & 39 & 17.8 & \\
& & & & & &
\end{tabular}


SUFFOIK QUADRATGLE-Continued.

Along Southern Ry. near Suffolk. (By H. J. Switzer in 1917-18.)

\begin{tabular}{|c|c|c|c|}
\hline Station. & Latitude. & Longitude. & $\begin{array}{l}\text { Distance } \\
\text { between }\end{array}$ \\
\hline 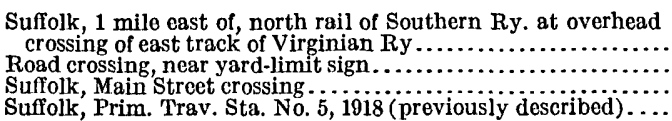 & $\begin{array}{ccc}\circ & \prime & \prime \prime \\
36 & 44 & 19.0 \\
36 & 44 & 05.9 \\
36 & 44 & 01.6 \\
36 & 44 & 16.5\end{array}$ & $\begin{array}{ccc}\circ & \prime \prime & \\
76 & 33 & 49.8 \\
76 & 34 & 31.4 \\
76 & 34 & 55.8 \\
76 & 34 & 58.4\end{array}$ & $\begin{array}{r}\text { Feet. } \\
3.031 \\
3,036 \\
1,521\end{array}$ \\
\hline
\end{tabular}

North along Norfolk Southern R. R. (By J. J. Phelan in 1918.)

North Carolina and Virginia State line, brass plate in granite monument on county road (Prim. Trav. Sta. No. 3) ..........

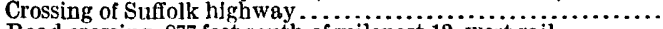

Road crossing, 877 feet south of milepost 12 , west rail. .........

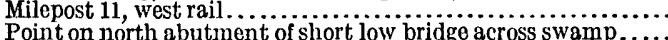

Point on north abutment of short low bridge across swamp....

Point on north abutment of bridge across swamp.............

Saunders, 56 feet north of station, at road crossing ............

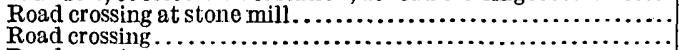

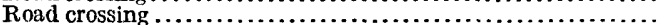

Crismond flag station, road crossing at, east rail .............

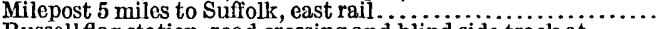

Russellflag station, road crossing and blind side track at...........

Road crossing, west rail . . . . . . . . . . . . . . . . . . . . . . . . . . .

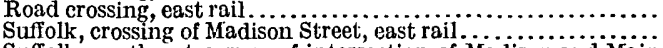

Suffolk, northeast corner of intersection of Madison and Main

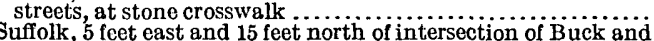

Suffolk, 5 feet east and 15 feet north of intersection of Buck and

Main streets............................................

Clerk's office; iron post stamped "Prim. Trav. Sta. No. 5 P,

1918",

\begin{tabular}{|lll|lll|r} 
& & & & & & \\
36 & 33 & 02.9 & 76 & 34 & 32.6 & $\ldots \ldots$, \\
36 & 33 & 16.0 & 76 & 34 & 33.2 & $\mathbf{1}, 322$ \\
36 & 34 & 07.8 & 76 & 34 & 38.4 & $\mathbf{5}, 253$ \\
36 & 35 & 08.6 & 76 & 34 & 42.7 & 6,160 \\
36 & 35 & 41.6 & 76 & 34 & 45.3 & 3,346 \\
36 & 37 & 02.7 & 76 & 34 & 46.4 & 8,205 \\
36 & 37 & 26.3 & 76 & 34 & 43.9 & 2,395 \\
36 & 38 & 15.9 & 76 & 34 & 43.1 & 5,011 \\
36 & 38 & 35.0 & 76 & 34 & 45.7 & 1,949 \\
36 & 38 & 52.0 & 76 & 34 & 48.1 & 1,722 \\
36 & 39 & 11.2 & 76 & 34 & 50.7 & 1,960 \\
36 & 39 & 43.2 & 76 & 34 & 55.3 & 3,256 \\
36 & 40 & 19.8 & 76 & 35 & 06.1 & 3,801 \\
36 & 41 & 09.8 & 76 & 35 & 17.2 & 5,140 \\
36 & 41 & 52.8 & 76 & 35 & 12.6 & 4,368 \\
36 & 42 & 51.6 & 76 & 34 & 45.0 & 6,358 \\
36 & 43 & 21.3 & 76 & 34 & 39.1 & 3,045 \\
& & & & & & \\
36 & 43 & 27.2 & 76 & 35 & 03.9 & $\mathbf{2 , 1 0 0}$ \\
36 & 43 & 46.8 & 76 & 34 & 59.0 & 2,029 \\
& & & & & & \\
36 & 44 & 16.5 & 76 & 34 & 58.4 & 2,999 \\
& & & & & & \\
\hline & & & & & &
\end{tabular}

\section{Northwest along highways.}

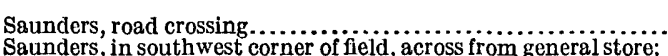

iron post stamped "Prim. Trav. Sta. No. 2, 1918"

On branch road west to Nurney, 40 feet west of center of Cora-

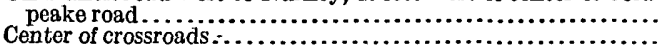

\begin{tabular}{|rrr|rrr|r}
36 & 38 & 15.9 & 76 & 34 & 43.1 & $\ldots \ldots \ldots$ \\
36 & 38 & 17.4 & 76 & 34 & 45.9 & 280 \\
36 & 38 & 06.0 & 76 & 35 & 01.1 & \\
36 & 38 & 14.7 & 76 & 36 & 40.4 & 1,691 \\
& & & & & &
\end{tabular}

Southwest along Atlantic Coast Line R. R.

Nurney, at station, 2.5 feet from north rail of road crossing of

Atlantic Coast Line R. R., on main track; iron post stamped

"Prim. Trav. Sta. No. 4 P, 1918"

Road crossing, northwest rail

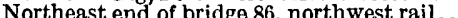

Culvert 87, northwest rail.

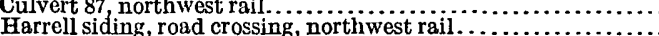

Milepost 29 , northwest rail opposite.

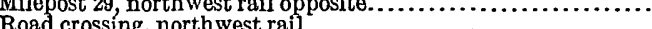

Whaley, 470 feet southwest of northeast end of station, at road

crossing, northwest rail.

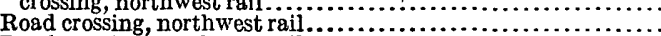

Road crossing, northwest rail.

Road crossing, northwest rail

Milepost 33 , northwest rail.

\begin{tabular}{|lll|l} 
& & & \\
& & & \\
36 & 38 & 32.6 & 76 \\
36 & 38 & 15.4 & 76 \\
36 & 38 & 01.8 & 76 \\
36 & 37 & 35.9 & 76 \\
36 & 37 & 04.4 & 76 \\
36 & 36 & 31.7 & 76 \\
36 & 35 & 53.4 & 76 \\
36 & 35 & 30.5 & 76 \\
36 & 35 & 06.4 & 76 \\
36 & 34 & 45.5 & 76 \\
36 & 34 & 33.2 & 76 \\
36 & 33 & 55.9 & 76 \\
& & &
\end{tabular}

\begin{tabular}{|lll|l} 
& & & \\
& & & \\
76 & 36 & 50.2 & 1,962 \\
76 & 37 & 09.2 & 2,322 \\
76 & 37 & 24.4 & 1,855 \\
76 & 37 & 53.2 & 3,518 \\
76 & 38 & 28.3 & 4,287 \\
76 & 39 & 04.6 & 4,439 \\
76 & 39 & 47.3 & 5,212 \\
76 & 40 & 12.7 & 3,104 \\
76 & 40 & 39.4 & 3,273 \\
76 & 41 & 02.7 & 2,842 \\
76 & 41 & 16.4 & 1,671 \\
76 & 41 & 57.8 & 5,068 \\
& & & \\
\hline
\end{tabular}

Magnetic declination for northwest part of quadrangle, $5.5^{\circ} \mathrm{W}$. 
SUFFOLK QUADRANGLE-Continued.

Southwest along Southern Ry. (By J. J. Phelan In 1918.)

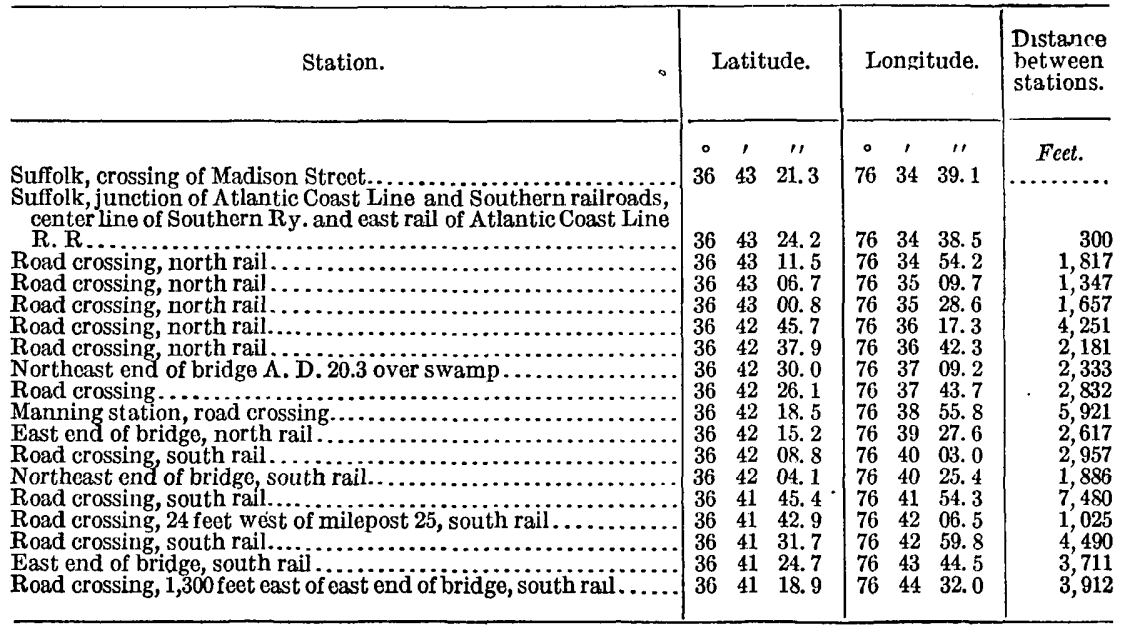

North along highways near west border of quadrangle.

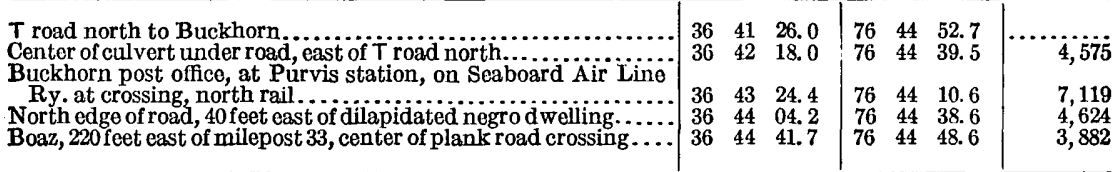

Magnetic declination for northwest corner of quadrangle, $5.5^{\circ} \mathrm{W}$.

SURRY QUADRANGLE.

On highways in north part of quadrangle. (By J. F. Wilson in 1916.)

\begin{tabular}{|c|c|c|c|c|c|c|c|}
\hline Station. & \multicolumn{3}{|c|}{ Latitude. } & \multicolumn{3}{|c|}{ Longitude. } & $\begin{array}{l}\text { Distance } \\
\text { between } \\
\text { stations. }\end{array}$ \\
\hline 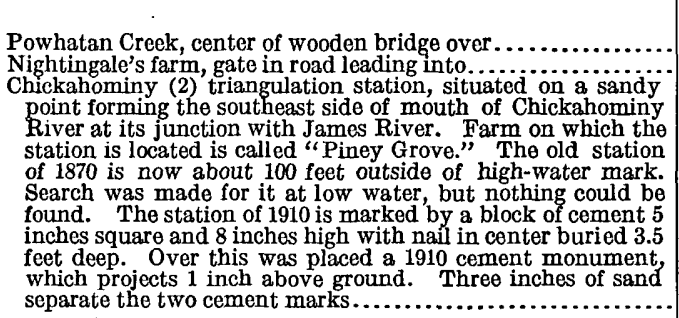 & $\begin{array}{c}\circ \\
37 \\
37\end{array}$ & $\begin{array}{c}\prime \\
14 \\
14\end{array}$ & 30.91 & $\begin{array}{c}\circ \\
76 \\
76\end{array}$ & $\begin{array}{l}1 \\
46 \\
51\end{array}$ & $\begin{array}{c}\prime \prime \prime \\
22.0 \\
29.8\end{array}$ & \begin{tabular}{c}
\multicolumn{2}{|c}{ Fect. } \\
$\ldots \ldots$ \\
$\cdots \ldots$
\end{tabular} \\
\hline
\end{tabular}

Magnetic declination for north part of quadrangle, $4^{\circ} 55^{\prime} \mathrm{W}$.

Northwest along highways in south part of quadrangle. (By J. J. Charters in 1918.)

\begin{tabular}{|c|c|c|c|c|c|c|c|}
\hline 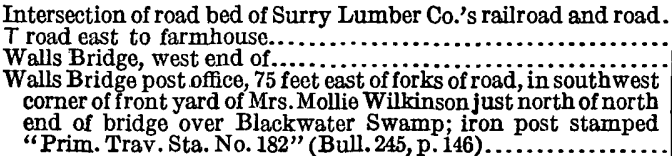 & $\begin{array}{l}37 \\
37 \\
37\end{array}$ & $\begin{array}{l}00 \\
00 \\
01\end{array}$ & $\begin{array}{l}09.0 \\
50.6 \\
26.4\end{array}$ & $\begin{array}{l}76 \\
76 \\
76\end{array}$ & $\begin{array}{l}51 \\
52 \\
52\end{array}$ & $\begin{array}{l}52.9 \\
24.2 \\
36.2\end{array}$ & $\begin{array}{l}4, \ddot{9 i} \\
3,742\end{array}$ \\
\hline
\end{tabular}


SURRY QUADRANGLE-Continued.

Northeast along highways in southeast corner of quadrangle.

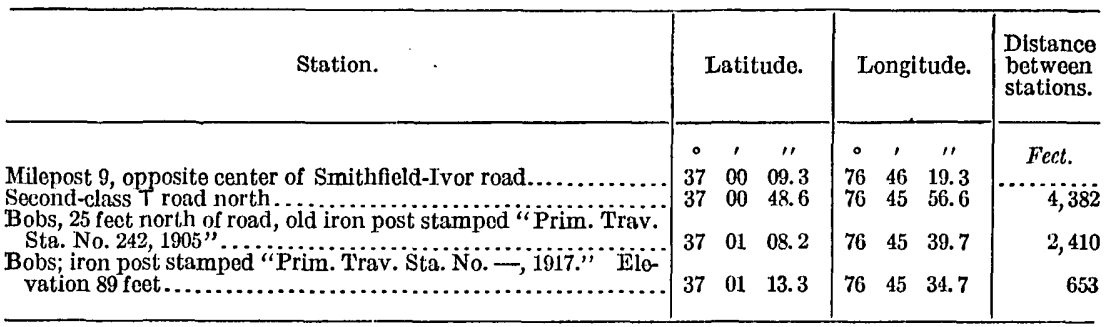

Along highways and Surry, Sussex \& Southampton Ry. from southwest corner to northeast corner of quadrangle. (By Olinus Smith in 1917.)

Crossroads, in southeast corner of; iron post stamped "Prim.

Trav. Sta. No. 132".......................................

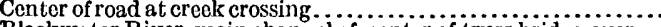

Blackwater River, main channel of, center of truss bridge over

Dendron, road crossing south of . ................................

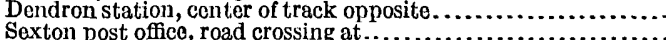

Sexton, 1 mile north of, 10 feet south of center of farm road, in

west bank of railroad; iron post stamped "Prim. Trav. Sta.

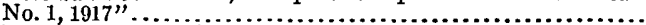

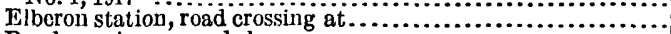

Road crossing, second class.

Moorings station, switchpost at, conter of track. . . . . . . . . . . . .

Road crossing, second class.

Surry station, 1,800 feet south of, in railroad $\dddot{Y}, 50$ feet east of

main track; iron post stamped "Prim. Trav. Sta. No. 2, 1917"

Road crossing, second class.

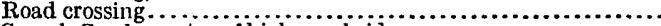

Crouch Creek, center of highway bridge over................

Jones triangulation station; recovered the old station mark 14

feet back from edge of bluff and 2 feet below surface. Placed

over it a cement monument, which projects 4 inches above the

surface..............................................

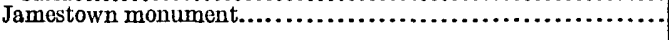

\begin{tabular}{|rll|lll|r}
37 & 00 & 47.5 & 76 & 59 & 39.6 & $\ldots \ldots$ \\
37 & 01 & 13.0 & 76 & 58 & 42.2 & 5,326 \\
37 & 01 & 34.2 & 76 & 52 & 43.3 & 5,234 \\
37 & 02 & 27.3 & 76 & 56 & 26.0 & 8,252 \\
37 & 02 & 45.5 & 76 & 55 & 52.5 & 3,286 \\
37 & 03 & 20.7 & 76 & 55 & 10.3 & 4,034 \\
& & & & & & \\
37 & 03 & 55.2 & 76 & 54 & 12.1 & 5,863 \\
37 & 04 & 33.3 & 76 & 53 & 10.6 & 6,305 \\
37 & 05 & 20.4 & 76 & 52 & 22.0 & 6,175 \\
37 & 05 & 56.1 & 76 & 51 & 45.2 & 4,681 \\
37 & 06 & 57.2 & 76 & 50 & 55.0 & 7,398 \\
& & & & & & \\
37 & 07 & 59.0 & 76 & 50 & 04.7 & 7,461 \\
37 & 08 & 42.5 & 76 & 49 & 42.1 & 4,769 \\
37 & 10 & 05.4 & 76 & 48 & 12.4 & 11,088 \\
37 & 10 & 36.4 & 76 & 46 & 56.0 & 6,939 \\
& & & & & & \\
& & & & & & \\
37 & 10 & 08.946 & 76 & 45 & 51.759 & 5,895 \\
37 & 12 & 31.48 & 76 & 46 & 39.90 & 14,033 \\
& & & & & & \\
\hline
\end{tabular}

Magnetic declination diagonally through quadrangle from southwest to northeast, $4^{\circ} 50^{\prime} \mathrm{W}$

TAPPAHANNOCK QUADRANGLE.

South along highways near east border of quadrangle. (By E. L. McNafr in 1916.)

\begin{tabular}{|c|c|c|c|c|c|c|c|}
\hline Station. & & Lati & ude. & & ong & tude. & $\begin{array}{l}\text { Distance } \\
\text { between }\end{array}$ \\
\hline 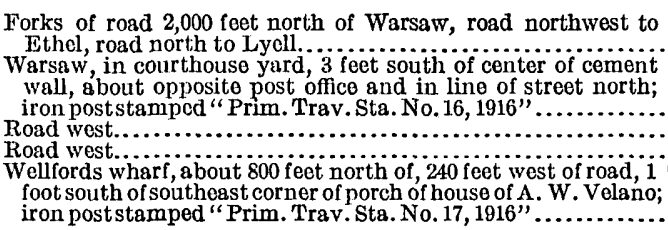 & $\begin{array}{r}\circ \\
37 \\
\\
\\
\\
37 \\
37 \\
37\end{array}$ & $\begin{array}{l}57 \\
55 \\
54\end{array}$ & $\begin{array}{c}\prime \prime \\
48.6 \\
29.9 \\
20.8 \\
05.4\end{array}$ & $\begin{array}{l}76 \\
76 \\
76\end{array}$ & $\begin{array}{l}45 \\
45 \\
45\end{array}$ & $\begin{array}{c}\prime \prime \\
19.4 \\
27.4 \\
00.2 \\
43.8 \\
51.8\end{array}$ & $\begin{array}{c}\text { Fect. } \\
(7,092) \\
\\
1,998 \\
(6,692) \\
5,243\end{array}$ \\
\hline
\end{tabular}

Along highways near north border of quadrangle (east half).

\begin{tabular}{|c|c|c|c|c|c|c|c|}
\hline 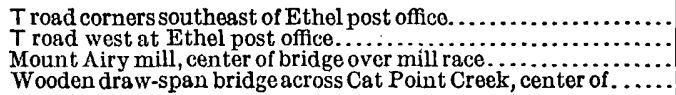 & $\begin{array}{l}37 \\
37 \\
37 \\
37\end{array}$ & $\begin{array}{l}59 \\
59 \\
58 \\
59\end{array}$ & $\begin{array}{l}38.3 \\
47.4 \\
59.1 \\
02.3\end{array}$ & $\begin{array}{l}76 \\
76 \\
76 \\
76\end{array}$ & $\begin{array}{l}46 \\
46 \\
47 \\
48\end{array}$ & $\begin{array}{l}39.6 \\
51.1 \\
52.5 \\
35.9\end{array}$ & $\begin{array}{r}1,297 \\
6,931 \\
3,488\end{array}$ \\
\hline
\end{tabular}


TAPPAHANNOCK QUADRANGLE-Continued.

South along highways through center of north half of quadrangle.

\begin{tabular}{|c|c|c|c|}
\hline Station. & Latitude. & Longitude. & $\begin{array}{l}\text { Distance } \\
\text { between } \\
\text { stations. }\end{array}$ \\
\hline 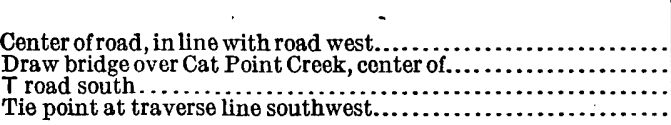 & $\begin{array}{ccc}\circ & \prime & \prime \prime \\
37 & 59 & 52.3 \\
37 & 58 & 28.7 \\
37 & 57 & 54.2 \\
37 & 57 & 43.9\end{array}$ & $\begin{array}{ccc}\circ & \prime & \prime \prime \\
76 & 51 & 20.1 \\
76 & 51 & 14.8 \\
76 & 50 & 12.0 \\
76 & 50 & 04.3\end{array}$ & $\begin{array}{l}\text { Feet. } \\
(2,559) \\
8,471 \\
6,116 \\
1,212\end{array}$ \\
\hline
\end{tabular}

East along highways through center of northeast quarter of quadrangle.

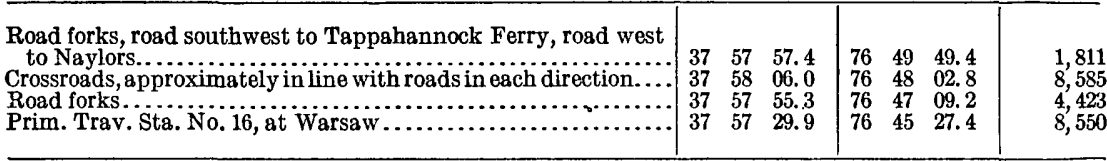

Magnetic declination near west border of quadrangle, $6^{\circ} 10^{\prime} \mathrm{W}$; along north border of quadrangle, $6^{\circ} 10^{\prime} \mathrm{W}$; t through center of north half of quadrangle, $6^{\circ} 06^{\prime} \mathrm{W}$; ; east through center of northeast quarter of quadrangle, $6^{\circ} 13^{\prime} \mathrm{W}$.

Along highways through east half of quadrangle from northwest to southeast.

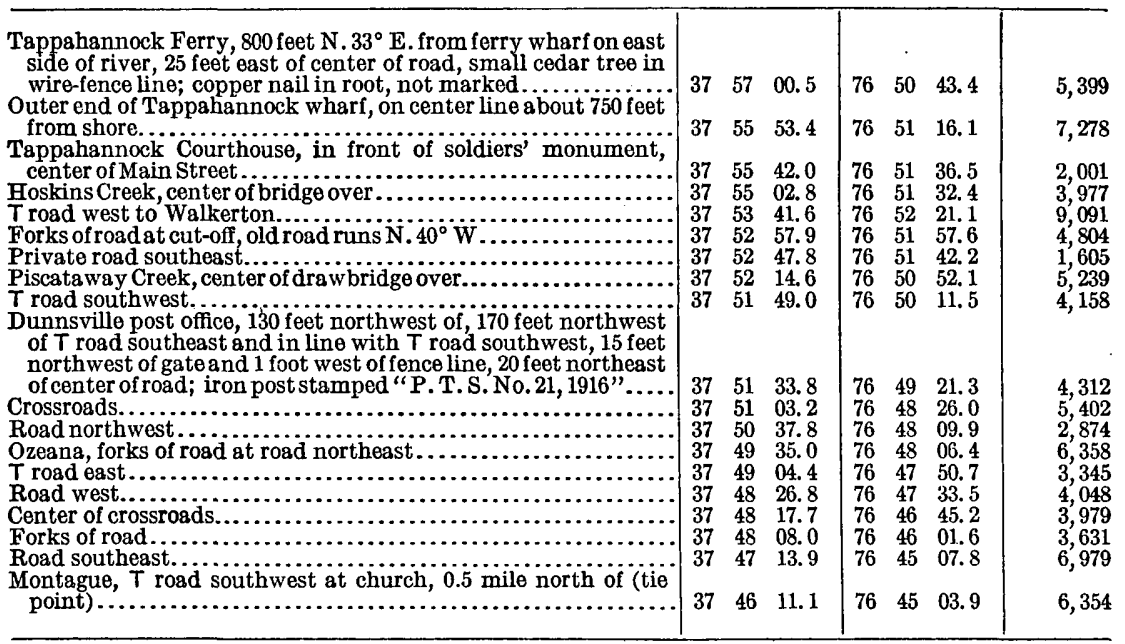

Along highways in southeast corner of quadrangle.

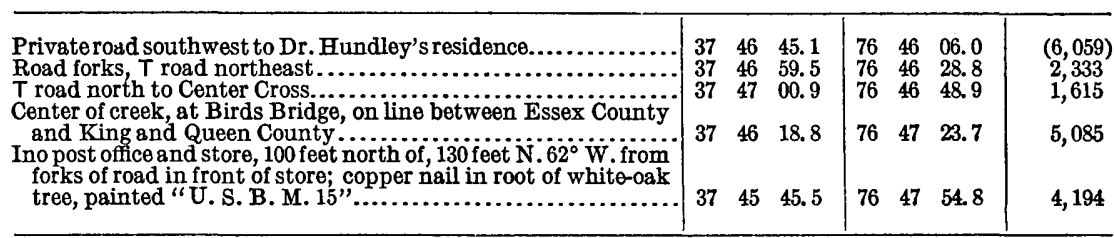

Magnetic declination for east half of quadrangle, $6^{\circ} 00^{\prime} \mathrm{W}$; for southeast corner of quadrangle, $5^{\circ} 43^{\prime} \mathrm{W}$. 
TAPPAHANNOCK QUADRANGLE-Continued.

East along highways near south border of quadrangle.

\begin{tabular}{|c|c|c|c|c|c|c|c|}
\hline . $\quad$ Station. & \multicolumn{3}{|c|}{ Latitude. } & \multicolumn{3}{|c|}{ Longitude. } & $\begin{array}{l}\text { Distance } \\
\text { between } \\
\text { stations. }\end{array}$ \\
\hline 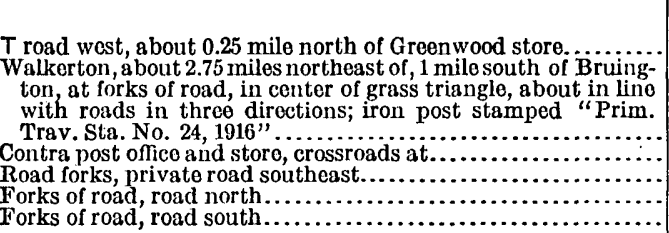 & $\begin{array}{l}37 \\
37 \\
37 \\
37 \\
37\end{array}$ & $\begin{array}{l}45 \\
45 \\
45 \\
46 \\
45\end{array}$ & $\begin{array}{l}20.6 \\
38.8 \\
52.6 \\
21.1 \\
56.8\end{array}$ & $\begin{array}{l}76 \\
76 \\
76 \\
76 \\
76\end{array}$ & $\begin{array}{l}59 \\
50 \\
50 \\
50 \\
49\end{array}$ & $\begin{array}{l}26.7 \\
4 \text { L. } 5 \\
34.1 \\
07.6 \\
0.8 .8\end{array}$ & \begin{tabular}{r}
1,087 \\
\hdashline,$\ldots 70$ \\
1,510 \\
5,471
\end{tabular} \\
\hline
\end{tabular}

Magnetic declination along south border of quadrangle, $5^{\circ} 50^{\prime} \mathrm{W}$.

North along highways near west border of quadrangle, south half.

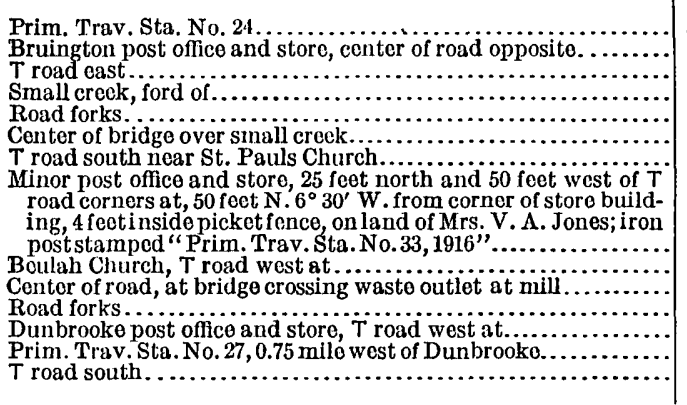

\begin{tabular}{|lll|lll|r}
\hline 37 & 45 & 20.6 & 76 & 59 & 26.7 & $\ldots \ldots \ldots$ \\
37 & 46 & 23.4 & 76 & 59 & 30.2 & $\mathbf{6}, 359$ \\
37 & 46 & 49.1 & 76 & 59 & 29.4 & 2,597 \\
37 & 47 & 37.2 & 76 & 59 & 03.8 & 5,274 \\
37 & 48 & 16.0 & 76 & 58 & 47.0 & 4,154 \\
37 & 48 & 25.4 & 76 & 58 & 29.3 & 1,710 \\
37 & 49 & 43.1 & 76 & 58 & 08.2 & 8,036 \\
& & & & & & \\
& & & & & & \\
37 & 50 & 22.4 & 76 & 58 & 58.9 & \\
37 & 51 & 54.9 & 76 & 58 & 40.7 & 5,680 \\
37 & 52 & 31.7 & 76 & 58 & 02.2 & 4,463 \\
37 & 52 & 41.8 & 76 & 57 & 49.9 & 1,420 \\
37 & 53 & 39.1 & 76 & 57 & 51.5 & 5,785 \\
37 & 53 & 56.8 & 76 & 58 & 39.1 & $\ldots \ldots$ \\
37 & 53 & 51.1 & 76 & 59 & 51.4 & 5,822 \\
& & & & & & \\
\hline
\end{tabular}

Magnetic declination along west border of south half of quadrangle, $7^{\circ} 00^{\prime} \mathrm{W}$.

Along highways from about center to the northwest corner of quadrangle.

\begin{tabular}{|c|c|c|c|c|c|c|c|}
\hline Tappahannock, soldiers' monument at & 37 & 55 & 4105 & 76 & 51 & 36.53 & \\
\hline 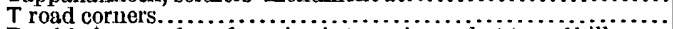 & 37 & 55 & 15.9 & 76 & 52 & 36.7 & \\
\hline Road sn's, wood road coming into main road at top of hill...... & 37 & 55 & 37.2 & 76 & 53 & 34.9 & 5,13 \\
\hline 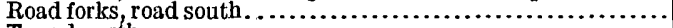 & 37 & 56 & 18.6 & 76 & 54 & 36.9 & 50 \\
\hline 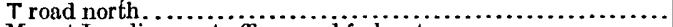 & 37 & 56 & 23.8 & 76 & 55 & 18.0 & 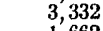 \\
\hline Mount Landing post office, road forks at. . & 37 & 56 & 35.7 & 76 & 55 & 32.3 & 1,66 \\
\hline Upper Mount Landing, T road north at. . & 37 & 56 & 50.4 & 76 & 56 & 19.2 & 04 \\
\hline Center of bridgo over small creek......... & 37 & 57 & 44.4 & 76 & 56 & 12.0 & \\
\hline T road nor theast, at top of hill, opposite old church. & 37 & 58 & 08.7 & 76 & 56 & 20.8 & 55 \\
\hline Road forks, road north $\ldots \ldots \ldots \ldots \ldots$ & 37 & 58 & 53.5 & 76 & 57 & 09.1 & 951 \\
\hline $\begin{array}{l}\text { Caret post office, opposite Crutchfields store, in center of triangle } \\
\text { at forks of road; copper nail in root of red-oak tree, painted }\end{array}$ & & & & & & & \\
\hline 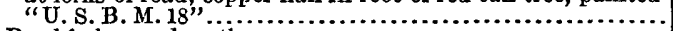 & 37 & 58 & 57.4 & 76 & 57 & 43.6 & 2,78 \\
\hline Road forks, road south. & 37 & 58 & 42.9 & 76 & 58 & 10.3 & \\
\hline T road corners, roads north, south, and east. . & 37 & 58 & 45.4 & 76 & 59 & 20.1 & 592 \\
\hline Road forks, in line of roads in three directions. ............. & 37 & 59 & 14.3 & 76 & 59 & 33.9 & 120 \\
\hline
\end{tabular}

Along highways near west border of quadrangle.

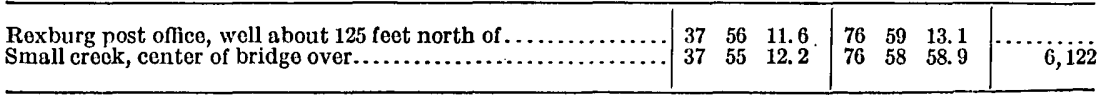

$121166^{\circ}-23-$ Bull. $709-32$ 
TAPPAHANNOCK QUADRANGLE-Continued.

East along highways through center of quadrangle.

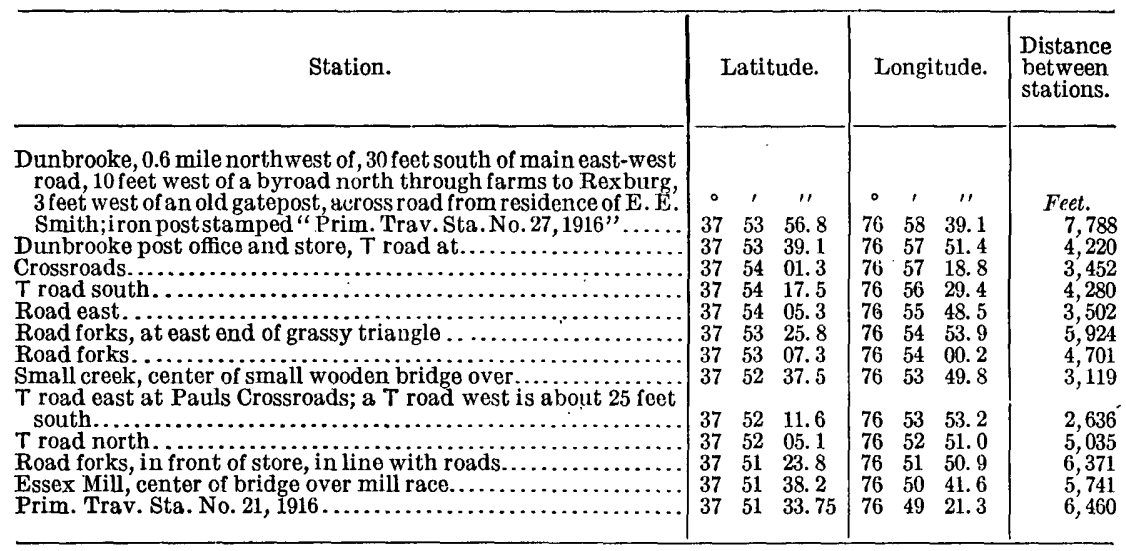

TOANO QUADRANGLE.

East along highways near north border of quadrangle.

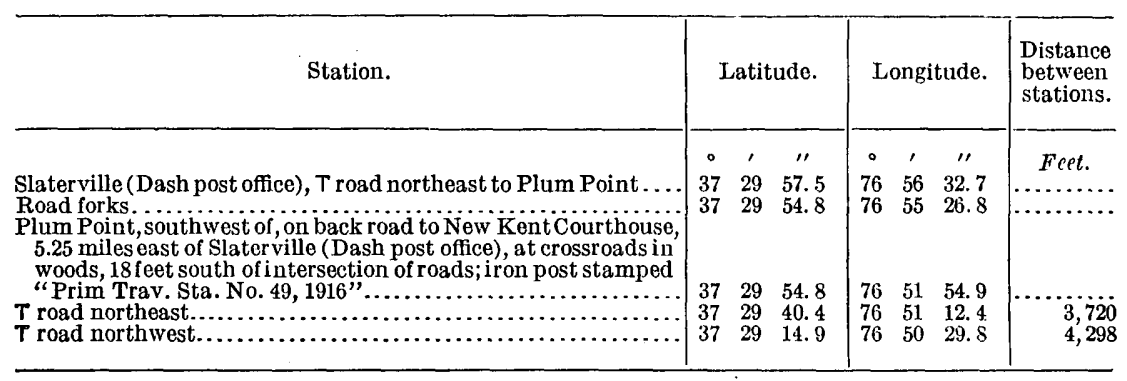

Magnetic declination along north border of quadrangle, $5^{\circ} 00^{\prime} \mathrm{W}$.

South along highways and Chesapeake \& Ohio Ry. near east border of quadrangle. (By J. H. Wison in 1916.)

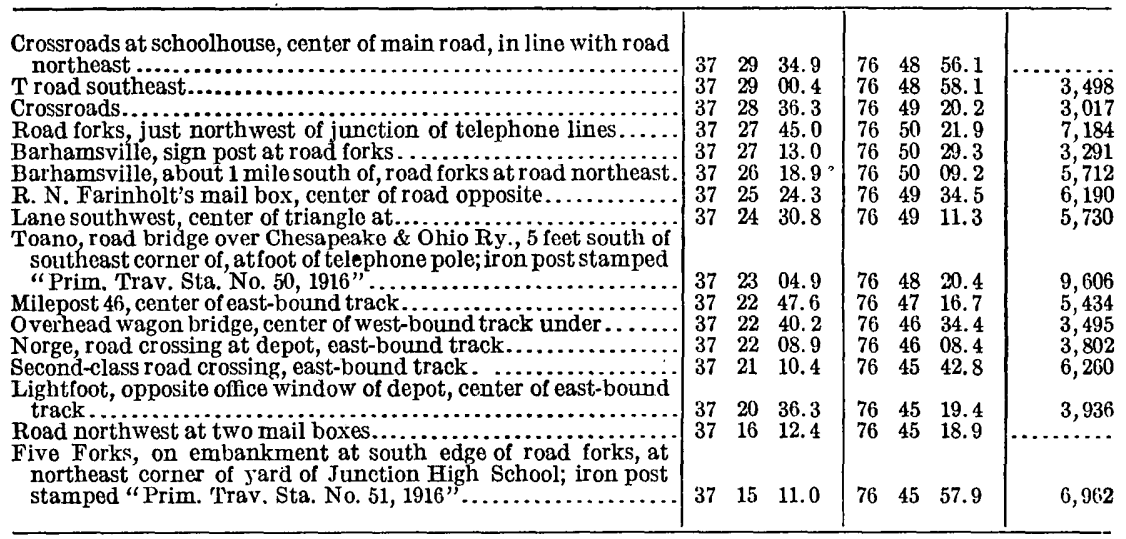

West along highways near south border of quadrangle.

\begin{tabular}{|c|c|c|c|c|c|c|c|}
\hline 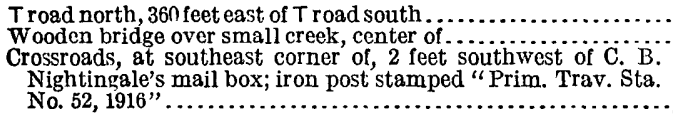 & $\begin{array}{l}37 \\
37 \\
37\end{array}$ & $\begin{array}{l}15 \\
15\end{array}$ & $\begin{array}{l}12.9 \\
18.6\end{array}$ & $\begin{array}{l}76 \\
76 \\
76\end{array}$ & $\begin{array}{l}47 \\
49\end{array}$ & $\begin{array}{l}58.3 \\
46.2 \\
31.1\end{array}$ & 3,916 \\
\hline
\end{tabular}


TOANO QUADRATGLE-Continued.

West along Chesapeake \& Ohio Ry. through center of quadrangle.

\begin{tabular}{|c|c|c|c|c|c|c|c|}
\hline \multirow[t]{2}{*}{ Station. } & \multicolumn{3}{|c|}{ Latitude. } & \multicolumn{3}{|c|}{ Longitude. } & \multirow{2}{*}{$\begin{array}{c}\begin{array}{c}\text { Distance } \\
\text { between } \\
\text { stations. }\end{array} \\
\text { Feet. }\end{array}$} \\
\hline & $\circ$ & ' & $\prime \prime$ & $\circ$ & , & I & \\
\hline Road crossing, 480 feet east of milepost 48 , west-bound track. & 37 & 23 & 40.5 & 76 & 48 & 09.6 & \\
\hline 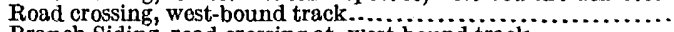 & 37 & 23 & 21.7 & 76 & 50 & 07.8 & 5,070 \\
\hline Branch Siding, road crossing at, west-bound track............. & 37 & 23 & 06.6 & 76 & 50 & 44.7 & 3,342 \\
\hline $\begin{array}{l}\text { Diascund, road crossing just east of depot, west-bound track...... } \\
\text { Diascund Creek, center of railroad bridge } 525 \text { over, west-bound }\end{array}$ & 37 & 23 & 39.9 & 76 & 52 & 14.6 & 7,998 \\
\hline track 60 fect east of southeast corner of depot, on south side of & 37 & 24 & 17.2 & 76 & 53 & 38.8 & 7,775 \\
\hline 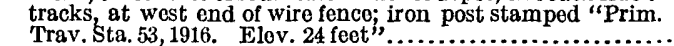 & 37 & 24 & 16.2 & 76 & & 41.4 & 5,053 \\
\hline Walker, road crossing at depot, west-bound track & 37 & 24 & 56.8 & 76 & 56 & 25.2 & 9,327 \\
\hline $\begin{array}{l}\text { Road crossing, about } 850 \text { feet wẹt of milepost } 57 \text {, west-bound } \\
\text { track }\end{array}$ & 37 & 25 & 31.7 & 76 & 58 & 09.6 & 9,129 \\
\hline $\begin{array}{l}\text { Windsor Shades, in front of office window of depot, west-bound } \\
\text { track } \ldots \ldots \ldots \ldots \ldots \ldots \ldots \ldots \ldots \ldots \ldots \ldots \ldots \ldots \ldots \ldots \ldots \ldots \ldots \ldots \ldots \ldots \ldots \ldots \ldots \ldots \ldots \ldots \ldots \ldots\end{array}$ & & & 40.2 & 76 & 58 & 59.1 & 4,085 \\
\hline
\end{tabular}

Along highways near west border of quadrangle.

\begin{tabular}{|c|c|c|c|c|c|c|c|}
\hline 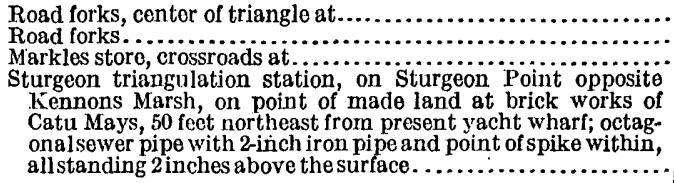 & $\begin{array}{l}37 \\
37 \\
37\end{array}$ & $\begin{array}{l}20 \\
20 \\
19\end{array}$ & $\begin{array}{l}45.4 \\
13.0 \\
32.2\end{array}$ & $\begin{array}{l}76 \\
76 \\
76\end{array}$ & $\begin{array}{l}59 \\
59 \\
59\end{array}$ & $\begin{array}{l}38.3 \\
33.0 \\
53.8\end{array}$ & $\begin{array}{l}3,304 \\
4,464\end{array}$ \\
\hline
\end{tabular}

Magnetic declination for east border of quadrangle, $5^{\circ} 09^{\prime} \mathrm{W}$.; for south border of quadrangle, $5^{\circ} 04^{\prime} \mathrm{W}$.

URBANNA QUADRANGLE.

Northwest along highways. (By E. L. MacNair in 1916.)

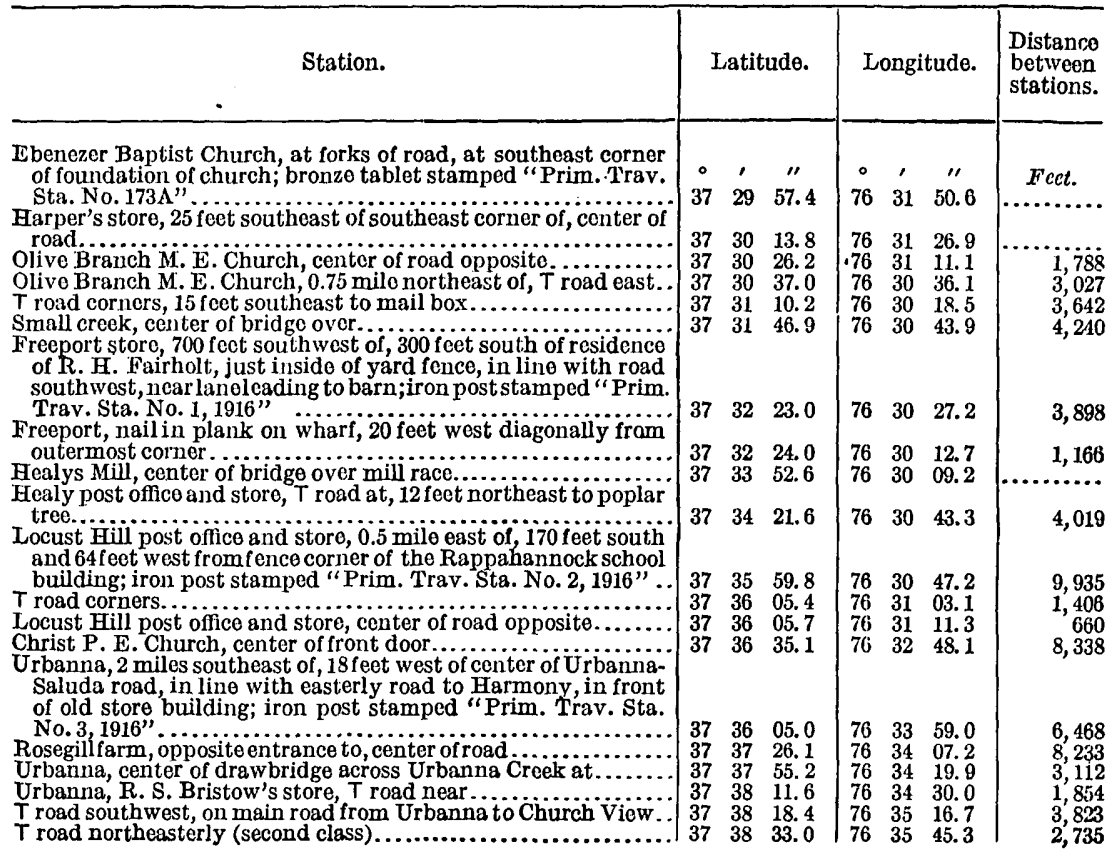


URBANNA QUADRANGLE-Continued.

Northwest along highways-Continued.

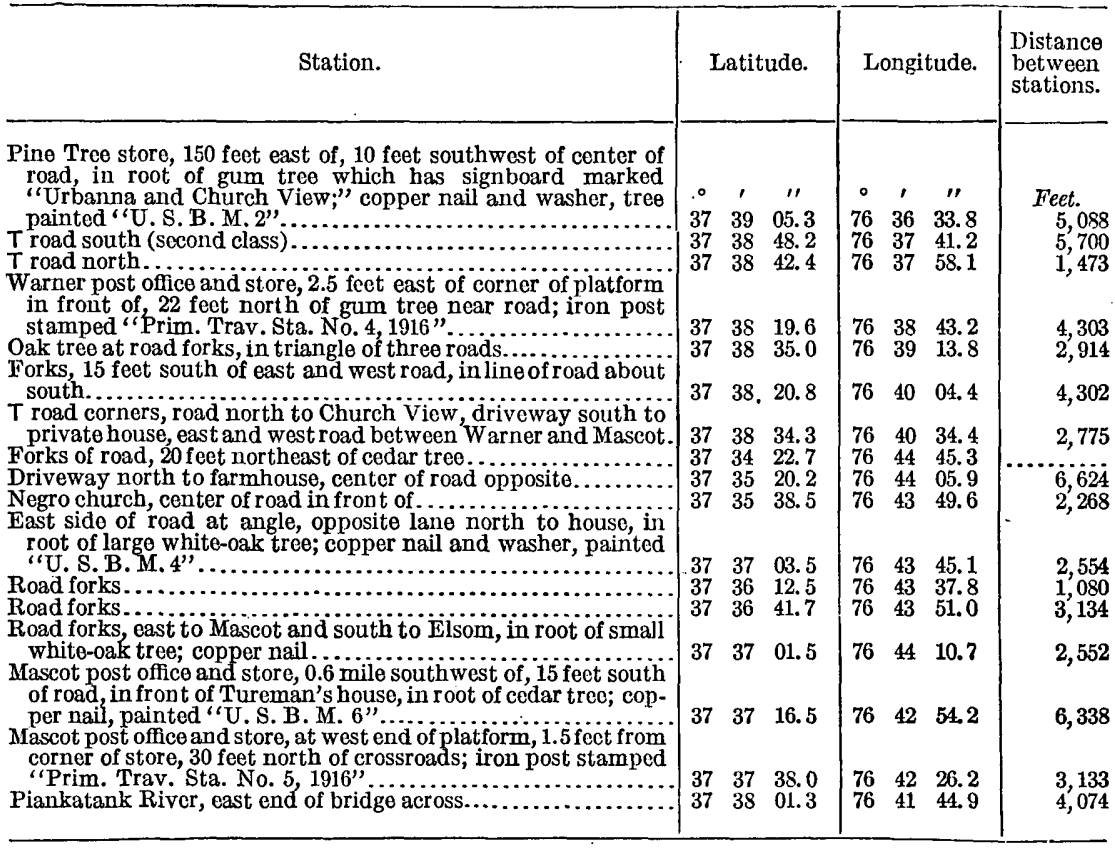

Magnetic declination for south half of east side of quadrangle, $5^{\circ} 59^{\prime} \mathrm{W}$.; east and west through middle of quadrangle, $5^{\circ} 36^{\prime} \mathrm{W}$.; for south half of west side of quadrangle, $5^{\circ} 24^{\prime} \mathrm{W}$.

West along highways on north border of quadrangle. (Checked spur line.)

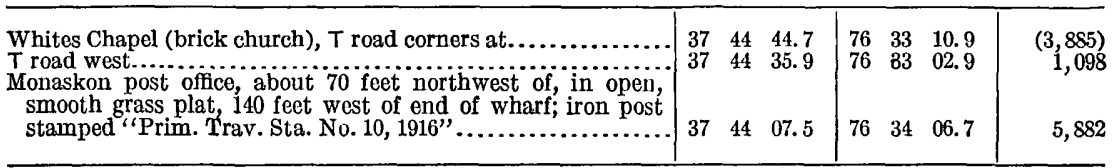

Magnetic declination along west border of quadrangle, $5^{\circ} 40^{\prime} \mathrm{W}$.

Southwest along highways in northwest quarter of quadrangle.

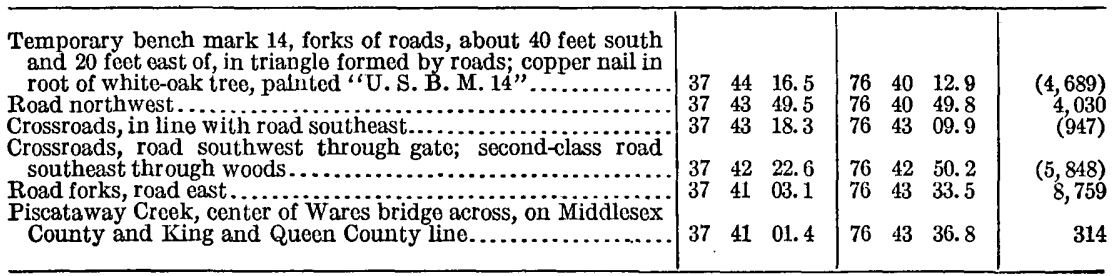

Along highways southeast through northwest quarter of quadrangle.

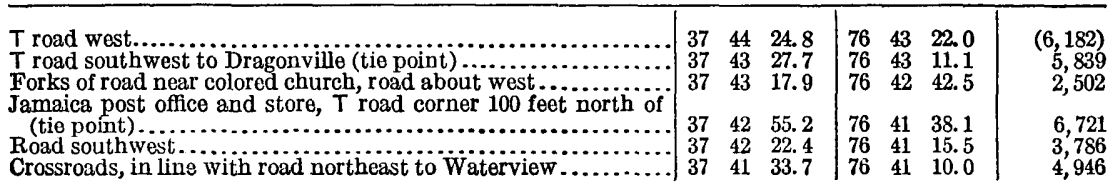


URBANNA QUADRANGLE-Continued.

Along highways southeast through northwest quarter of quadrangle-Continued.

\begin{tabular}{|c|c|c|c|c|c|c|c|}
\hline Station. & \multicolumn{3}{|c|}{ Latitude. } & \multicolumn{3}{|c|}{ Longitude. } & $\begin{array}{l}\text { Distanco } \\
\text { between } \\
\text { stations. }\end{array}$ \\
\hline 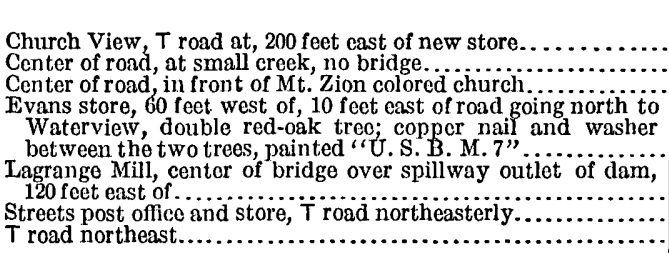 & $\begin{array}{l}37 \\
\\
37 \\
37 \\
39\end{array}$ & $\begin{array}{l}1 \\
40 \\
40 \\
40 \\
\\
40 \\
39 \\
39 \\
39\end{array}$ & $\begin{array}{l}4 \prime \prime \\
40.4 \\
27.5 \\
18.6 \\
14.6 \\
52.4 \\
38.2 \\
17.6\end{array}$ & $\begin{array}{c}\circ \\
76 \\
76 \\
76 \\
\\
76 \\
\\
76 \\
76 \\
76\end{array}$ & $\begin{array}{c}\prime \\
40 \\
40 \\
40 \\
\\
39 \\
\\
38 \\
37 \\
36\end{array}$ & $\begin{array}{c}\prime \prime \\
45.8 \\
17.0 \\
01.4 \\
\\
19.8 \\
\\
25.5 \\
08.8 \\
50.6\end{array}$ & $\begin{array}{r}\text { Feet. } \\
5,733 \\
1,552 \\
\\
3,364 \\
4,913 \\
6,330 \\
2,553\end{array}$ \\
\hline
\end{tabular}

Along highways near west border of quadrangle.

Road forks, at north end of triangle formed by roads.

Road forks, road south to Velma, road west to Ware's Chirch.

Road forks, at west end of triangle formed by roads, station is in line with road southwest to Little Plymouth and road

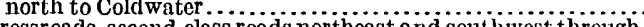

Crossroads, second-class roads nor theast and sout hwest through fields...

Velma store and post office, 40 feet southeast of, 20 fect west of road, on root of white-oak tree; copper nail and washer, tree

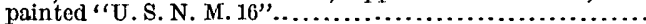

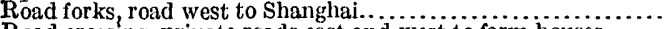

Road crossing, private roads east and west to farm houses.....

\begin{tabular}{|lll|lll|l}
37 & 40 & 11.9 & 76 & 44 & 17.3 & 5,342 \\
37 & 39 & 42.8 & 76 & 43 & 54.7 & 3,459 \\
& & & & & & \\
37 & 39 & 16.3 & 76 & 44 & 04.5 & 2,782 \\
37 & 38 & 58.5 & 76 & 43 & 13.0 & $4,51.5$ \\
& & & & & & \\
37 & 38 & 46.7 & 76 & 42 & 46.7 & 2,423 \\
37 & 38 & 23.3 & 76 & 43 & 08.5 & $2,9.48$ \\
37 & 37 & 56.6 & 76 & 42 & 49.7 & 3,090 \\
& & & & & & \\
\hline
\end{tabular}

Magnetic declination for northwest quarter of quadrangle, $5^{\circ} 33^{\prime} \mathrm{W}$.; for west border of quadrangle, $5^{\circ} 35^{\prime} \mathrm{W}$.

VIRGINIA BEACH QUADRANGLE.

East along highways and north along electric road. (By F. J. McMaugh in 1917.)

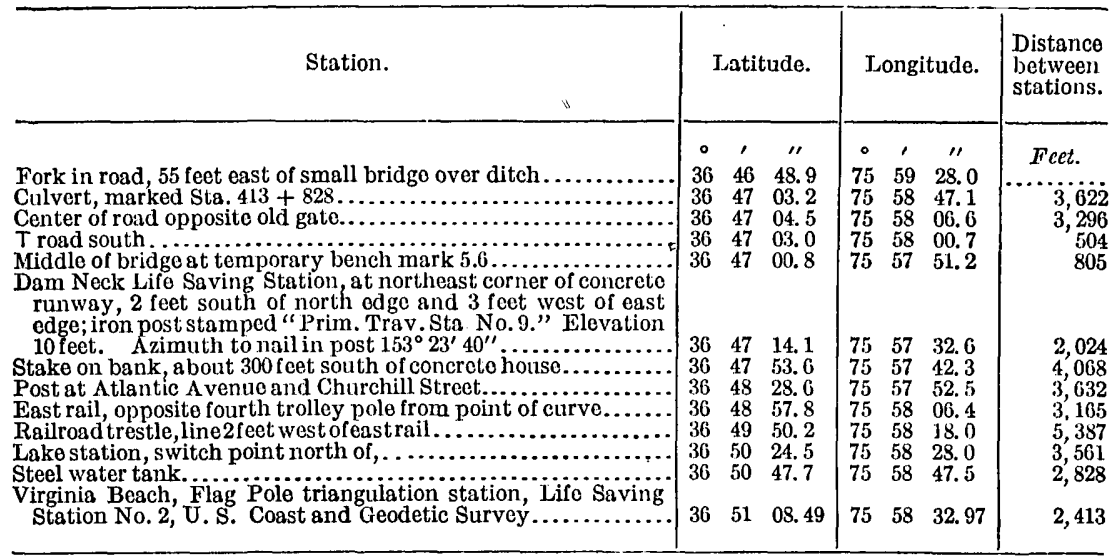

Magnetic declination for quadrangle, $5.8^{\circ} \mathrm{W}$. 
WELIVIILE QUADRANGLE.

East along highways near south border of quadrangle. (By J. C. Fales in 1918.)

\begin{tabular}{|c|c|c|c|c|c|c|c|}
\hline \multirow[t]{2}{*}{ Station. } & \multicolumn{3}{|c|}{ Latitude. } & \multicolumn{3}{|c|}{ Longitude. } & \multirow{2}{*}{$\begin{array}{l}\begin{array}{l}\text { Distance } \\
\text { between } \\
\text { stations. }\end{array} \\
\text { Feet. }\end{array}$} \\
\hline & $\circ$ & , & & $\circ$ & , & $\prime \prime$ & \\
\hline Road fork north. & 37 & 00 & 05.2 & 77 & 57 & 00.1 & \\
\hline 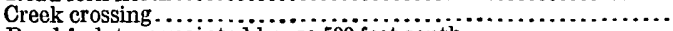 & 37 & 00 & 16.6 & 77 & 56 & 23.3 & 3,2006 \\
\hline Road fork to unpainted house 500 feet south ............ & 37 & 00 & 19.0 & 77 & 56 & 01.4 & 1,789 \\
\hline Creek crossing, 600 feet east of colored schoolhouse $\ldots \ldots \ldots \ldots$ & 37 & 00 & 31.5 & 77 & 55 & 33.2 & 2,615 \\
\hline $\begin{array}{l}\text { Crossing of road to Harper's home with a road from Black- } \\
\text { stone; hub at northwest corner of } \ldots \ldots \ldots \ldots \ldots \ldots \ldots \ldots \ldots\end{array}$ & 37 & 00 & 35.1 & & & 48.2 & 3,667 \\
\hline 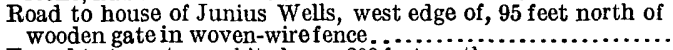 & 37 & 00 & 27.9 & 77 & 54 & 00.6 & 3,923 \\
\hline $\begin{array}{l}\text { Troad to two-story white house } 200 \text { feet south............... } \\
T \text { lane to two-story white house } 1,000 \text { feet south, three metal }\end{array}$ & 37 & 00 & 19.3 & 77 & 53 & 26.4 & 2,910 \\
\hline 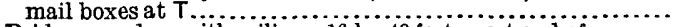 & 37 & 00 & 08.8 & 77 & 53 & 03.5 & 2,135 \\
\hline Bridge, wooden, with railings, 16 by 40 feet, west end of . . . . . . . & 37 & 00 & 14.8 & $\begin{array}{l}77 \\
77\end{array}$ & 51 & 13.4 & $\cdots \cdots$ \\
\hline I road to house $01 \mathrm{R}$. A. Lckels 200 leet north . ................ & & & & & & & \\
\hline
\end{tabular}

Magnetic declination for south border of quadrangle, $4^{\circ} 17^{\prime} \mathrm{W}$.

WHITE PLAINS QUADRANGLE.

South along highways near east border of quadrangle. (By J. C. Fales In 1918.)

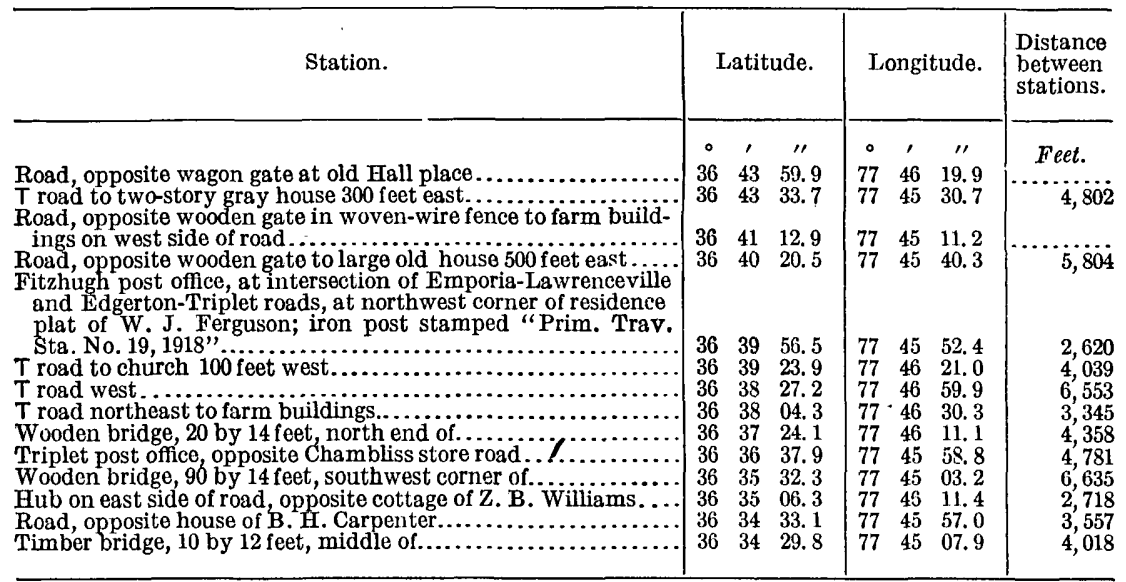

Magnetic declination for east border of quadrangle, $3^{\circ} 50^{\prime} \mathrm{W}$.

Southwest along Southern Ry. through northwest corner of quadrangle. (By J. C. Fales in 1918.)

\begin{tabular}{|c|c|c|c|c|c|c|c|}
\hline Milepost 100 , south rail opposite $\ldots \ldots \ldots \ldots \ldots \ldots \ldots \ldots \ldots \ldots \ldots \ldots \ldots \ldots$ & \multicolumn{3}{|c|}{$\begin{array}{lll}36 & 44 & 27.1\end{array}$} & \multicolumn{2}{|c|}{$77 \quad 55$} & 57.5 & \\
\hline Meherrin River bridge, at east end of trestle approach, south & & & & & & & \\
\hline 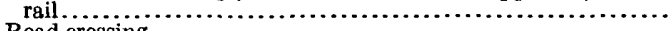 & 36 & 44 & 05.0 & 77 & 56 & 51.2 & 4,906 \\
\hline 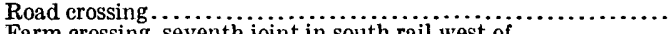 & 36 & 44 & 08.4 & 77 & 57 & 36.8 & 3,725 \\
\hline Farm crossing, seventh joint in south rail west of $\ldots \ldots \ldots \ldots \ldots$ & 36 & 43 & 54.0 & 77 & 58 & 59.4 & 6,879 \\
\hline
\end{tabular}

South and southwest along highways. (By J. C. Fales, in 1918.)

Prim. Trav. Sta. No. 16,1918 (previously described in Lawrenceville quadrangle) ................................................

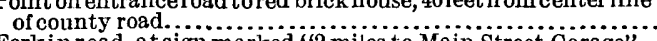
Forkin road, at sign marked " 2 miles to Main street Garage"...

Troad south to two-story house.......................... $36 \quad 43 \quad 59.3$

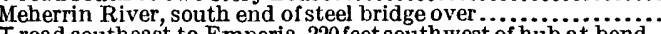

$T$ road southeast to Emporia, 220 feet south west of hub at bend, in road wherelane runs northeast. 
WHITE PLAIN̂S QUADRANGLE-Continued.

South and southwest along highways-Continued.

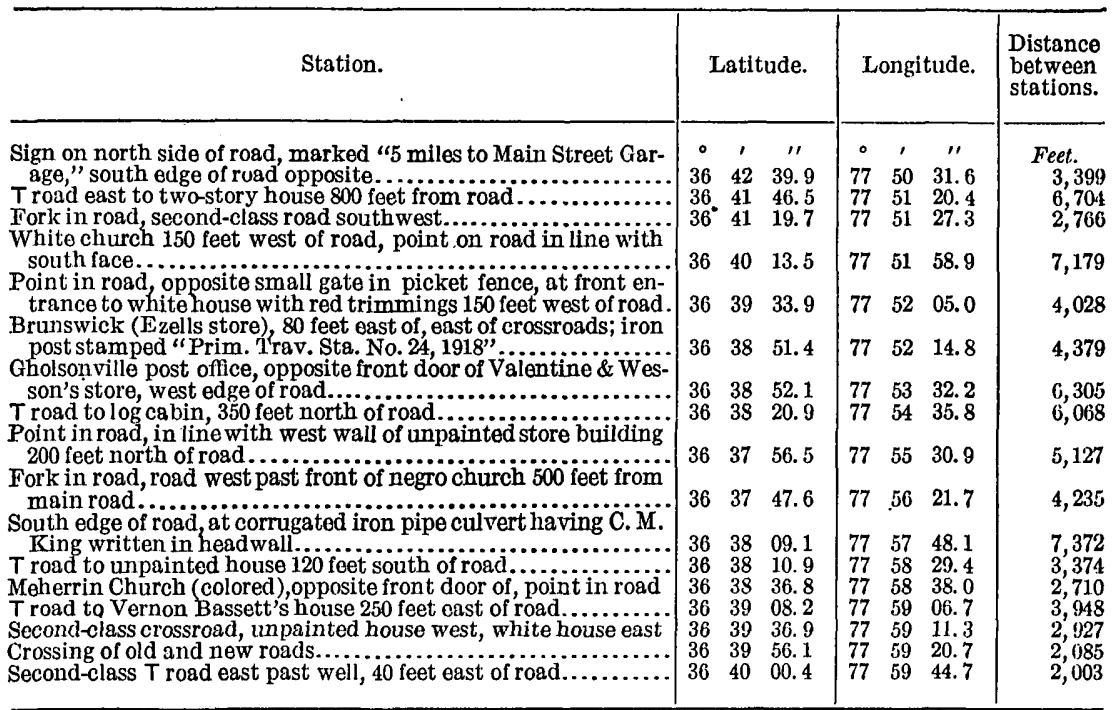

North along highways. (By H. J. Switzer in 1918.)

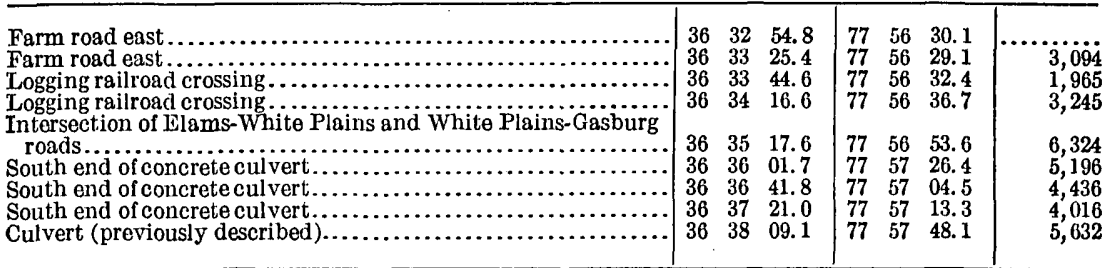

(By J. C. Fales In 1918.) Prim. Trav. Sta. No. 24,1918 (previously described) ...............................

$\begin{array}{llll} & \\ \end{array}$

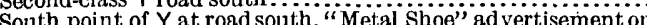
15-inch pine tree at southwest corner of intersection.

Point in road, opposite woven-wire gate atr oad to unpainted

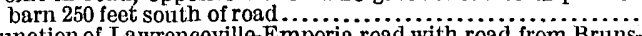

Junction of Lawrenceville-Emporia road with road from Bruns: wick...................................

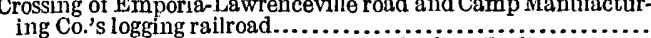

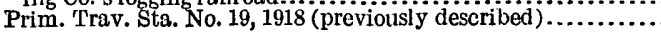

\begin{tabular}{|lll|lll|r}
36 & 38 & 51.4 & 77 & 52 & 14.8 & $\ldots \ldots \ldots$ \\
36 & 38 & 48.5 & 77 & 51 & 29.7 & 3,687 \\
36 & 38 & 42.3 & 77 & 50 & 46.8 & 3,559 \\
36 & 38 & 51.3 & 77 & 49 & 36.2 & 5,825 \\
36 & 39 & 08.8 & 77 & 49 & 12.3 & 2,631 \\
36 & 40 & 01.3 & 77 & 48 & 31.4 & 6,280 \\
36 & 40 & 06.6 & 77 & 47 & 54.4 & 3,062 \\
36 & 39 & 57.5 & 77 & 47 & 28.8 & 2,279 \\
36 & 39 & 56.47 & 77 & 45 & 52.33 & 7,867 \\
\hline
\end{tabular}

Magnetic declination for center of quadrangle, $3^{\circ} 43^{\prime} \mathrm{W}$; for south border of quadrangle, $4^{\circ} 22^{\prime} \mathrm{W}$.; for west border of quadrangle, $4^{\circ} 33^{\prime} \mathrm{W}$.; for east border of quadrangle, $4^{\circ} 33^{\prime} \mathrm{W}$.

See also Emporia quadrangle. 
WILIIAMSBURG QUADRANGLE.

South along Chesapeake \& Ohio Ry. and highways in southwest portion of quadrangle. (By J. H. Wilson in 1916.)

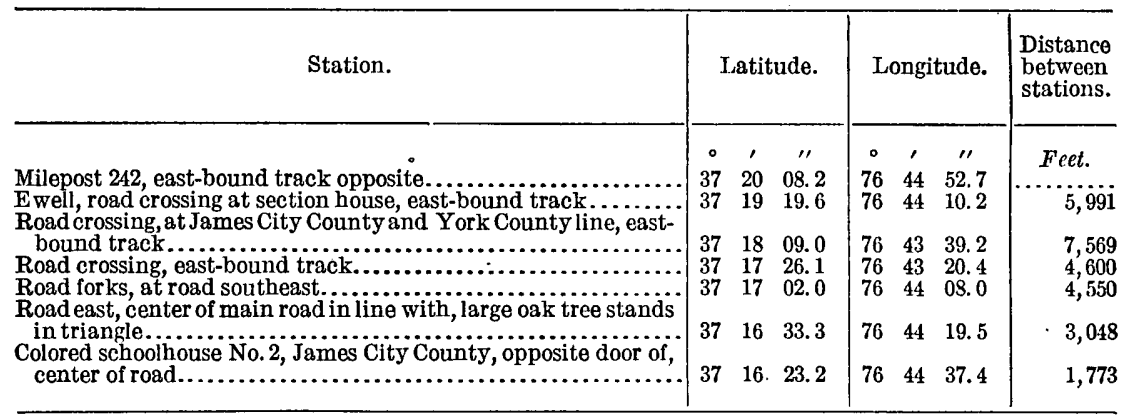

Magnetic declination for southwest part of quadrangle, $4^{\circ} 55^{\prime} \mathrm{W}$.

Along highways. (By J. J. Charters in 1918.)

\begin{tabular}{|c|c|c|c|c|c|c|c|}
\hline 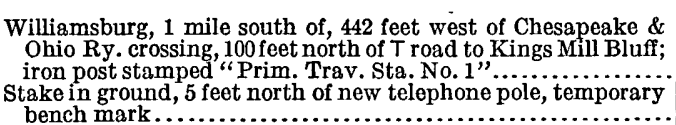 & 37 & 16 & 00.12 & \multicolumn{2}{|c|}{$\begin{array}{ll}76 & 40\end{array}$} & 53.05 & \multirow{4}{*}{$\begin{array}{r}2,380 \\
1,428 \\
1,796\end{array}$} \\
\hline 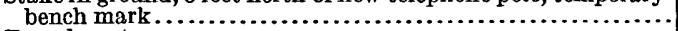 & 37 & 15 & 36.88 & 76 & 40 & 57.68 & \\
\hline 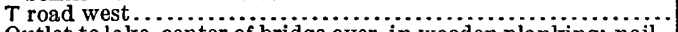 & 37 & 15 & 26.53 & 76 & 41 & 09.69 & \\
\hline $\begin{array}{l}\text { Outlet to lake, center of bridge over, in wooden planking; nail... } \\
\text { Grove station, about } 2 \text { miles northwest of, } 50 \text { feet from railroad }\end{array}$ & 37 & 15 & 08.77 & 76 & 41 & 10.07 & \\
\hline 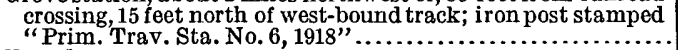 & 37 & 15 & 07.05 & 76 & 39 & 46.00 & \\
\hline 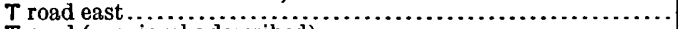 & 37 & 15 & 31.73 & 76 & 39 & 11.47 & \\
\hline 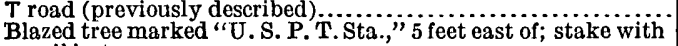 & 37 & 15 & 38.14 & 76 & 39 & 52.38 & \\
\hline 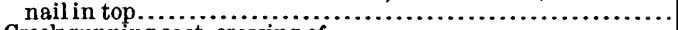 & 37 & 15 & 01.70 & 76 & 38 & 02.68 & \\
\hline Creek running & 37 & 15 & 03.02 & 76 & 37 & 58.68 & \\
\hline northwest (previously described)... & 37 & 15 & 19.68 & 76 & 37 & 55.43 & 1,70 \\
\hline aonument marked "W. A." ..... & 37 & 15 & 11.68 & 76 & 39 & 47.93 & \\
\hline T rog & 37 & 15 & 14. 90 & 76 & 39 & & \\
\hline ich of Chesapeake \& Ohio Ry & 37 & 15 & 34.57 & 76 & 39 & 51.51 & $\mathbf{1}, 98$ \\
\hline T road............. & 37 & 15 & 38.14 & 76 & 39 & 52.38 & 36 \\
\hline T road south ....... & 37 & 15 & 47.05 & 76 & 39 & 55.67 & \\
\hline T road north at L. C. White's & 37 & 15 & 50.55 & 76 & 39 & 57. & 38 \\
\hline T road west $\ldots \ldots \ldots \ldots \ldots \ldots \ldots$ & 37 & 15 & 57.31 & 76 & 40 & 25.62 & 2,37 \\
\hline T road southwest........ & 37 & 15 & 56.27 & 76 & 40 & 45. 50 & 1,61 \\
\hline Prim. Trav. Sta. No. 1 (previously described) & 37 & 16 & 00.12 & 76 & 40 & & 72 \\
\hline Intersection of crossroads, at 11 mail boxes.... & 37 & 15 & 06.04 & 76 & 37 & & \\
\hline T road north & 37 & 15 & 08.50 & 76 & 37 & 27.34 & \\
\hline 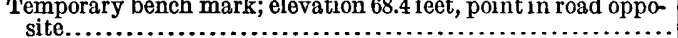 & 37 & 15 & & 76 & 37 & & 1,19 \\
\hline Road south near temporary bench mark; elevation 85.6 feet..... & 37 & 15 & 12.41 & 76 & 37 & & \\
\hline 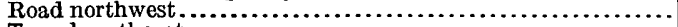 & 37 & 15 & 19.68 & 76 & 37 & 55. & \\
\hline$T$ road southeast..... & 37 & 15 & 25.10 & 76 & 38 & 14. 06 & 1,603 \\
\hline T road south ...... & 37 & 15 & 29.46 & 76 & 38 & & \\
\hline T road north. & 37 & 15 & & 76 & 38 & & \\
\hline T road northwest to house. $\ldots \ldots \ldots$ & 37 & 15 & 37.94 & 76 & 38 & 39.88 & 1,141 \\
\hline Dupont powder mill, on branch railroad to, 15 feet southeast of & & & & & & & \\
\hline $\begin{array}{l}\text { south rail, } 25 \text { feet southeast of crossroads; iron post stamped } \\
\text { "Prim. Trav. Sta. No.11, } 1918 \text { " } \ldots \ldots \ldots \ldots \ldots \ldots \ldots \ldots \ldots \ldots \ldots \ldots \ldots \ldots\end{array}$ & 37 & 15 & 40.58 & 76 & 38 & 52.88 & 1,084 \\
\hline
\end{tabular}

Magnetic declination for south border of quadrangle, $5.5^{\circ} \mathrm{W}$. 
YORETOWN QUADRANGLE.

CAMP ABRAHAM EUSTIS.

Along highways. (By J. J. Charters, in 1918.)

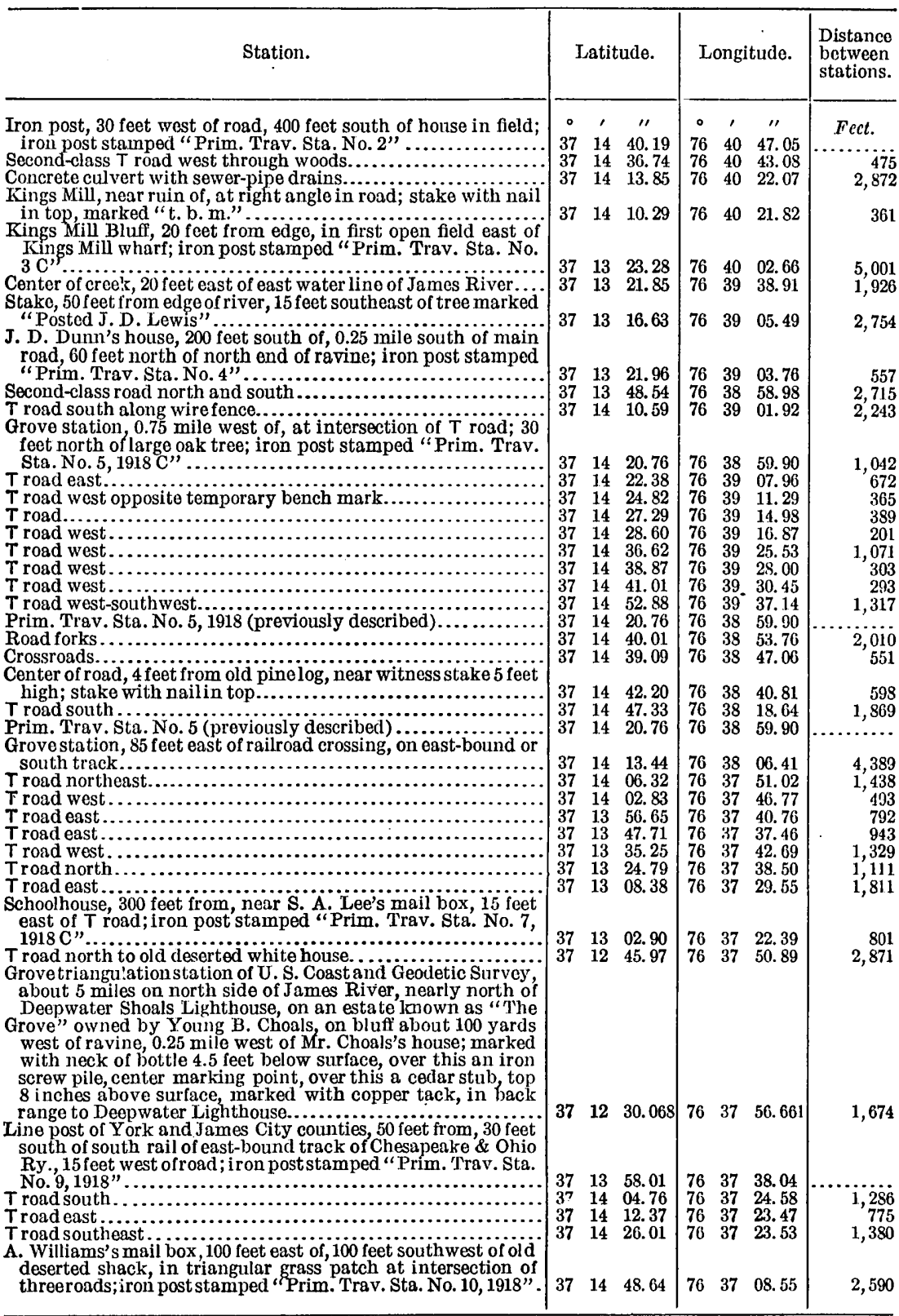

Magnetic declination for north border of quadrangle, $5.5^{\circ} \mathrm{W}$. 
YORKTOWN QUADRANGLE-Continued.

Northeast along highways near center of quadrangle. (By J. C. Fales in 1918.)

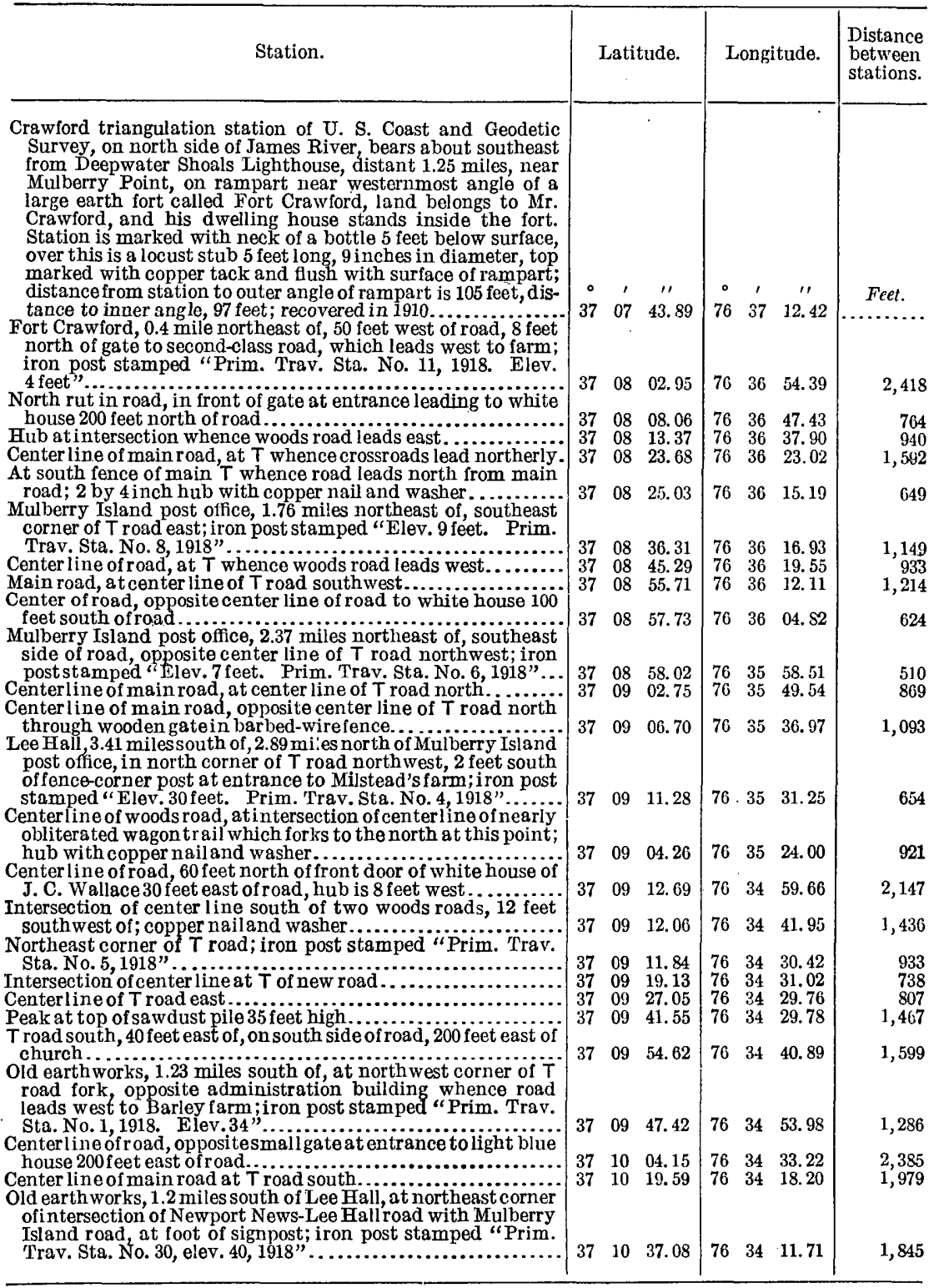

Magnetic declination for center of quadrangle, $5.5^{\circ} \mathrm{W}$.

Checked spur line from Primary Traverse Station No. 1 to Primary Traverse Station No. 2. (By J. C. Fales in 1918.)

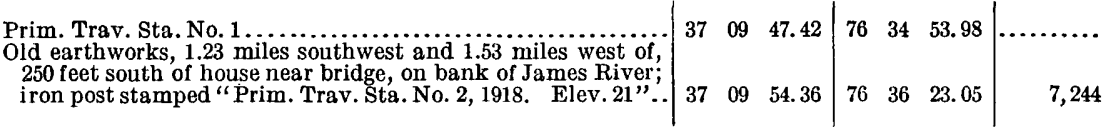


YORKTOWN QUADRANGLE-Continued.

From Primary Traverse Station No. 4 northwest to Primary Traverse Station No: 3, thence south to Primary Traverse Station No. 8.

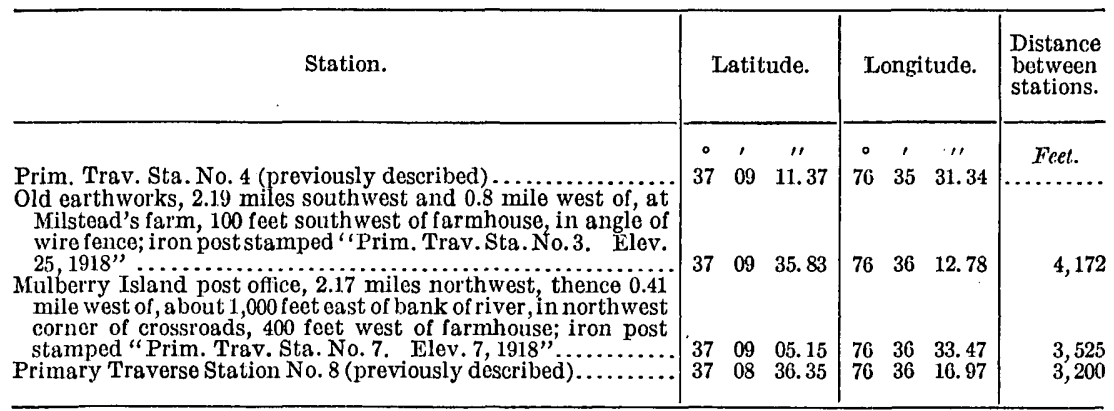

From Primary Traverse Station No. 5 via Shipyard Landing to Primary traverse Station No. 3. (By J. C. Fales and J. J. Phelan in 1918.)

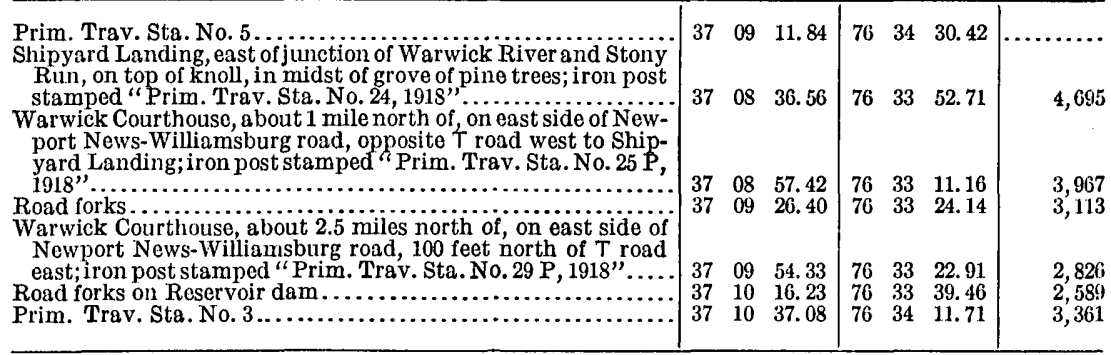

From Grove triangulation station via highways to Primary Traverse Station No. 3 at old earthworks. (By J. J. Charters in 1918.)

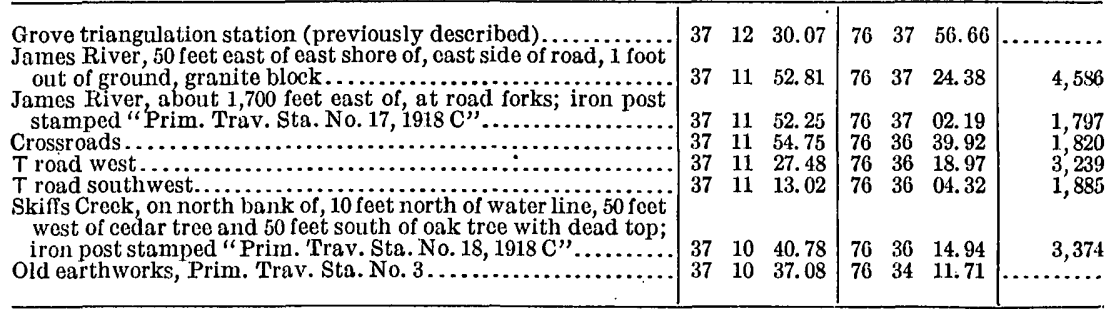

\section{From Primary Traverse Station No. 7 along highways to Primary Traverse Station No. 3.}

\begin{tabular}{|c|c|c|c|c|c|c|c|}
\hline $\begin{array}{l}\text { Prim. Trav. Sta. No. } 7 \text { (previously described) } \ldots \ldots \ldots \ldots \ldots \ldots \\
\text { Morning Star Church, in triangular patch of grass, at T road }\end{array}$ & & & & & 37 & 22.39 & \\
\hline south; iron post stamped "Prim. Trav. Sta. No.14, 1918 C".. & 37 & 19 & & 76 & 36 & & \\
\hline 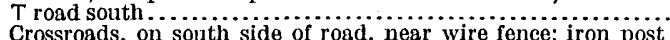 & 37 & 11 & 56.61 & 76 & 35 & 58.25 & 3,199 \\
\hline 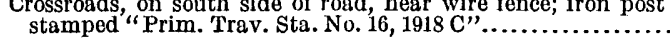 & 37 & 11 & 47.12 & 76 & 35 & 34.74 & 2,129 \\
\hline
\end{tabular}


YORKTOWN QUADRANGLE-Continued.

\author{
MULBERRY ISLAND.
}

Along highways. (By J. C. Fales in 1918.)

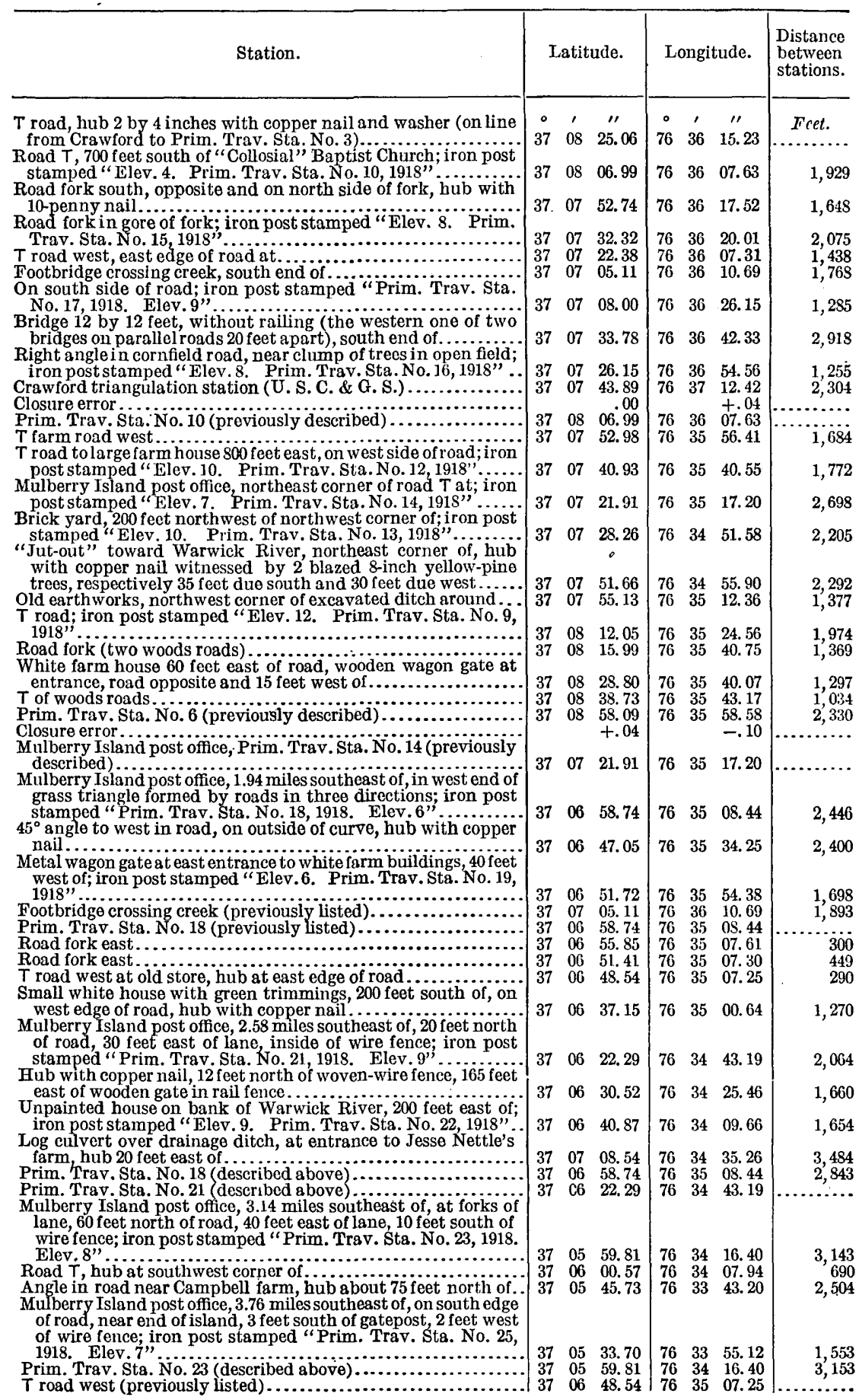


YORKTOWN QUADRANGLE-Continued.

MULBERRY ISL $\Lambda$ ND-Continued.

Along highways-Continued.

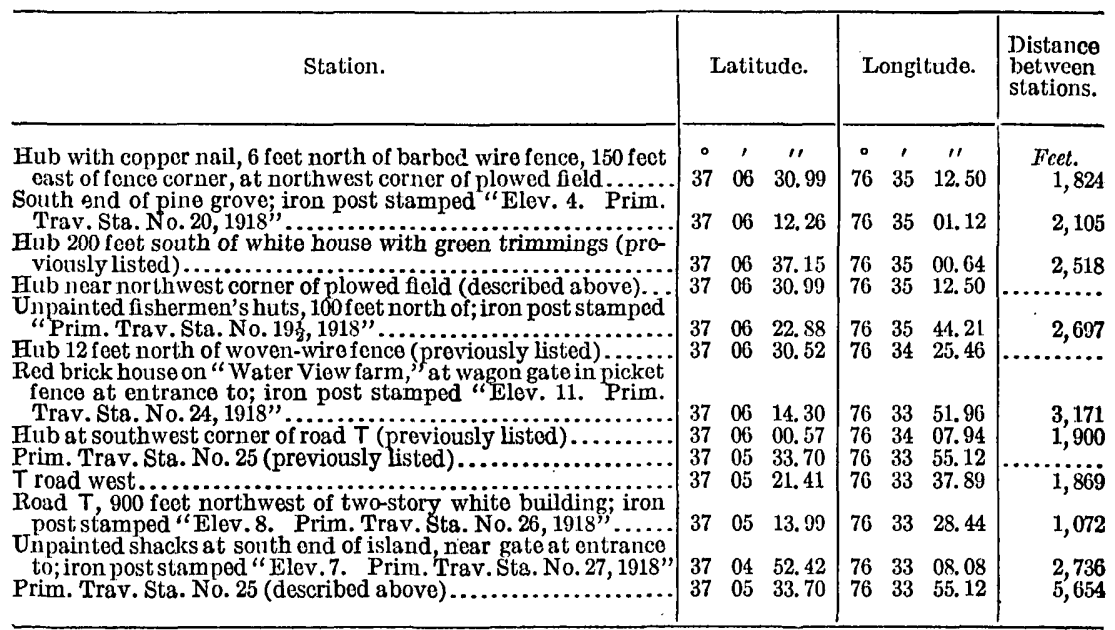

Along highways. (By J. J. Phelan in 1918.)

Prim. Trav. Sta. No. 25

Troad west.

Warwick couri........................................ num tablet 36 "

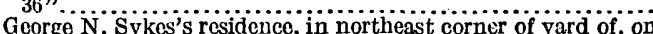
south side of T road and on west side of main Newport NewsWilliamsburg road from Denbigh post office to Mennonite colony iron post stamped "Prim. Trav. Sta. No. 27 P, 1918"

Crossroads, center of, nail in hub

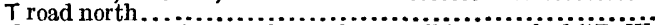

T road south, 18 feet northwest from mail box marked “ $\dddot{\mathrm{P}}$. $\dddot{w}$.

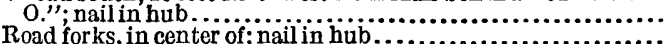

Hoops machineshop, 30 feet west of, 2.5 feet east of gatepost, on

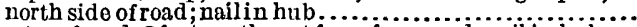

Center of road, 8 feet northwest from farm road; nail in hub....

Henry Schelley's residence, in corner of field, 5 feet west of center of road where wood road leaves farm road; iron post stamped "Prim. Trav. Sta. No. 23 P, 1918".

Hub in ground 17 feet north of tree marked "P. $\stackrel{T}{ } .17$ feet, ; and 19 feot east of tree marked "P. T. 19 feet"....................

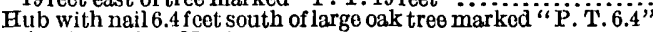
Prim. Trav. Sta. No. 24

\begin{tabular}{rrr|rrr|r}
37 & 08 & 57.42 & 76 & 33 & 11.16 & $\ldots \ldots \ldots, \ldots$ \\
37 & 08 & 36.53 & 76 & 32 & 53.21 & 2,566 \\
& & & & & & \\
37 & 07 & 47.21 & 76 & 32 & 32.76 & 5,257 \\
& & & & & & \\
37 & 07 & 38.54 & 76 & 32 & 22.00 & 1,237 \\
37 & 07 & 30.88 & 76 & 32 & 44.57 & 1,537 \\
37 & 07 & 29.50 & 76 & 33 & 14.78 & 2,450 \\
37 & 07 & 28.35 & 76 & 33 & 27.60 & 1,050 \\
37 & 07 & 29.82 & 76 & 33 & 48.60 & 1,700 \\
37 & 07 & 26.01 & 76 & 34 & 06.10 & 1,440 \\
37 & 07 & 43.27 & 76 & 33 & 52.60 & 2,060 \\
& & & & & & \\
37 & 08 & 04.78 & 76 & 34 & 22.32 & 3,245 \\
37 & 08 & 11.50 & 76 & 34 & 14.51 & 930 \\
37 & 08 & 29.28 & 76 & 33 & 51.71 & 2,577 \\
37 & 08 & 36.56 & 76 & 33 & 52.71 & 750 \\
& & & & & \\
\hline
\end{tabular}

Spur line along highways.

Jesse Nettler's residence, 70.9 feet southwest from chimney of, 46.2 feet south from nail in 5 -inch walnut tree, and 28.6 feet north from nail in 6-inch pine tree; iron post stamped " Prim. Trav. Sta. No. 22 P, 1918

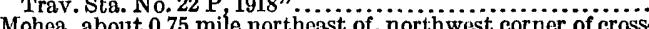

theast of, northwest corner of crossroads, in field about 3 feet from fence line; iron post stamped "Prim. Trav. Sta. No. 21P, 1918".

Mench vilie, about 0.75 mile northwest of in field at northeast corner of junction of Cedar road and Youngs road; iron post stamped "Prim. Trav. Sta. No. 20 P, 1918".................

\begin{tabular}{|rrr|rrr|r}
37 & 07 & 02.21 & 76 & 33 & 36.76 & $\ldots \ldots \ldots$ \\
37 & 06 & 36.82 & 76 & 32 & 45.86 & 7,077 \\
37 & 05 & 34.98 & 76 & 32 & 04.98 & 3,037 \\
\hline
\end{tabular}

Southeast along highways near north border of quadrangle. (By J. J. Charters in 1018.)
Prim. Trav. Sta. No. 7 (previously located)

Near $T$ road bearing west, on root of white oak tre..............

box (Lincoln Oronge) U' S. temporary bench mark........

U. S. temporary bench mark marked " $76.6 " \ldots \ldots \ldots \ldots \ldots \ldots \ldots$

U. S. temporary bench mark marked " 76.6 road south, on south side of road, near mail box of $\mathbf{C}$.

Cole, U. S. bench mark marked " 63.3 Elev".

\begin{tabular}{rrr|rrr|r}
37 & 13 & 02.90 & 76 & 37 & 22.39 & $\ldots \ldots \ldots \ldots$ \\
37 & 12 & 48.42 & 76 & 37 & 10.06 & \\
37 & 12 & 31.79 & 76 & 36 & 48.53 & 1,770 \\
37 & 12 & 25.09 & 76 & 36 & 35.49 & 2,421 \\
& & 1,253
\end{tabular}


YORKTOWN QUADRANGEI-Continued.

Southeast along highways near north border of quadrangle-Continued.

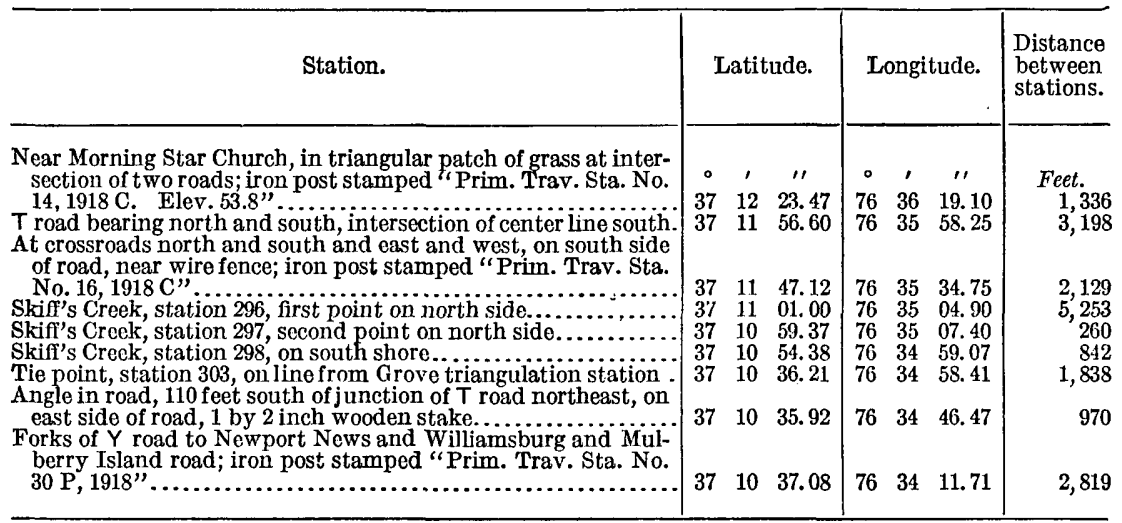

East along highways near north border of quadrangle. (By J. J. Charters in 1018.)

On Yorktown road near Charlos Corner; U. S. permanent bench

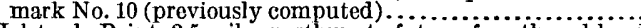

Halsteads Point, 2.5 miles northwest of, top of north end head wall of concrete culvert over small creek; chiseled square marked "U. S. B. M. 39.2".

Halsteads Point, 2.25 miles northwest of, 0.25 mile east of small creek, 350 feet west of dim road crossing highway, 4 feet from telephone pole 2593, at break of hill, top of 4-inch pine stake;

$\mathrm{T}$ roper nail and washer, marked " $\mathrm{U}$. S. B. $\mathrm{M} .87 .4$. $\ldots \ldots \ldots \ldots$

Halsteads Point, 1.8 miles northwest of, on north side of Williamsburg-Yorktown road, on east side of T road north; iron post stamped "Prim. Trav. Sta. 12 P, 85 1918," corner fence

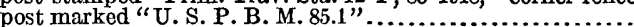

Halsteads Point, 1.5 miles northwest of, top of south end of stone pipe culvert, telephone pole 20 feet west marked "U. S. 82.4"

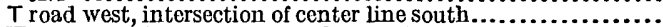

Halsteads Point, 1.2 miles northwest of, on summit of low ridge, on south side of road, opposite $T$ road north, in root of 40 -inch oak tree; copper nail and washer, tree marked "U. S. B. M. $82.9^{\prime \prime}$.

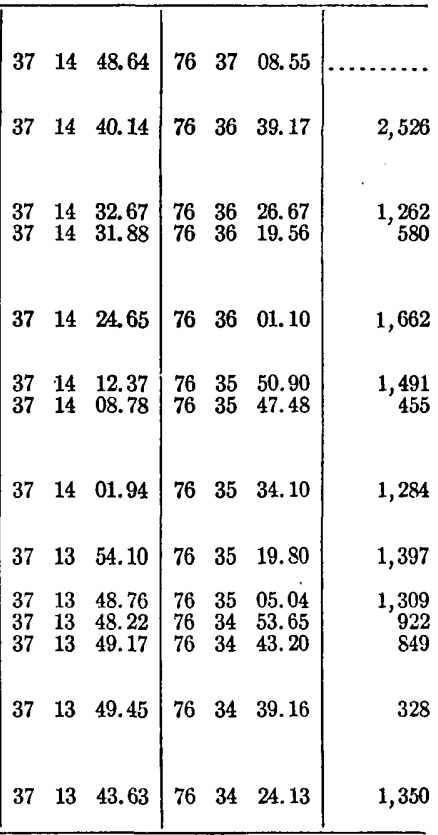

alsteads Point 0.9 mile northwest of, on south side of road, at $T$ road north, in root of 15-inch pine tree; 40-penny wire nail,

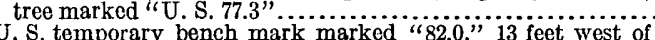
center of road

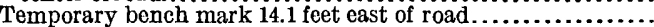

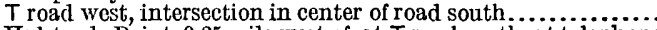

Halsteads Point, 0.25 mile west of, at $T$ road north, at telephone pole 2679 , top of 3 -inch stake; wire nail, pole marked "U. S. 83.1"

Halsteads Point, at intersection of three roads; Yorktown, Wi\}liamsburg, and Newport News, near Goodrich guidepost, in triangular grass plat; iron post stamped "Prim. Trav. Sta.

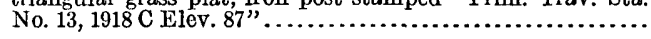

Magnetic declination for north border of quadrangle, $5.4^{\circ} \mathrm{W}$.

West along highways near north border of quadrangle. (By J. J. Phelan in 1918.)

Yorktown monument, U. S. Coast \& Geodetic Survey triangu-

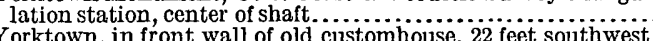

Yorktown, in front wall of old customhouse, 22 feet southwest

of road crossing, U. S. G. S. bench mark ...................... west of courthouse, 25 fect north of Yorktown road.............
Road, 29 feet south of edge of, 30 feet southeast from edge of bridge; nailin hub efo

Yorktown, 1.6 miles west of, at crossroads, at fork of Dabney road, 80 feet north-northwest of pine tree marked with white paint; iron post stamped "Prim. Trav. Sta. No. 33 P, 1918. Elev. $69 "$.

\begin{tabular}{|lll|lll|r}
37 & 14 & 00.23 & 76 & 30 & 18.84 & $\ldots \ldots \ldots$ \\
37 & 14 & 05.24 & 76 & 30 & 28.62 & 939 \\
37 & 14 & 09.27 & 76 & 30 & 33.86 & 587 \\
37 & 14 & 20.67 & 76 & 30 & 48.37 & 1,645 \\
& & & & & & \\
37 & 13 & 58.01 & 76 & 31 & 57.03 & 6,006 \\
37 & 14 & 02.61 & 76 & 32 & 25.96 & 2,386 \\
& & & & & & \\
37 & 13 & 58.83 & 76 & 32 & 43.90 & 1,500
\end{tabular}

York 37 miles west of in southwest corner of T south through woods; iron pipe 2 inches in diameter with cap on top painted white; top of cap marked "U. S. 75.5 B. M.".. $\mid \begin{array}{llllll}37 & 13 & 58.83 & 76 & 32 & 43.90\end{array}$ 
YORKTOWN QUADRANGLE-Continued.

west along highways near north border of quadrangle-Continued.

\begin{tabular}{|c|c|c|c|}
\hline Station. & Latitude. & Iongitude. & $\begin{array}{l}\text { Distance } \\
\text { between } \\
\text { stations. }\end{array}$ \\
\hline 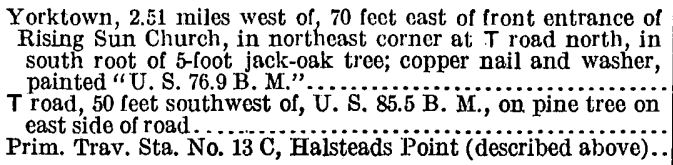 & $\begin{array}{ccc}\circ & , & \prime \prime \\
37 & 13 & 56.17 \\
37 & 13 & 49.88 \\
37 & 13 & 43.63\end{array}$ & $\begin{array}{ccc}\circ & \prime & \prime \prime \\
76 & 32 & 53.48 \\
76 & 34 & 07.90 \\
76 & 34 & 24.13\end{array}$ & $\begin{array}{r}\text { Fcet. } \\
819 \\
6,052 \\
1,456\end{array}$ \\
\hline
\end{tabular}

Magnetic declination for north border of quadrangle, $5.6^{\circ} \mathrm{W}$.

East along highways near center of quadrangle. (By J. J. Charters In 1918.)

Prim. Trav. Sta. No. 14 (previously described)

Lebanon Church, 1.6 miles southwest of, in southwest angle of crossroads, at north end of a grove of black-locust trees, at entrance to old farmhouse, top of 2 by 4 inch stake; copper nail and washer tree 50 fectsouthwest marked "U. S. B. M. 46.1"

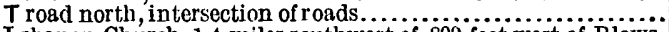

Lebanon Church, 1.4 miles southwest of, 800 feet west of Blows Mill Run, at break of hill, on south side of road, 1 foot from fence post, top of 3-inch stake; copper nail and washer, post marked "U. S. 52.0"

Lebanon Church, 1.0 mile southwest of, 40 feet south of Chesapeake \& Ohio Ry, track, on east side of road, top of 7-inch oak stake; copper nail and washer, telegraph pole 10 feet south marked "U. S. B. M. 49.3"

Lebanon Church, 0.5 mile west of, on north side of road, at $T$ road north, top of 14-inch pine stump; copper nail and washer, troe 40 leet west marked "U. S. B. M. 84.3" ...................

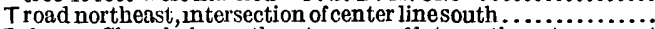

Lebanon Church, in southeast corner of lot, northwest corner at junction of first-class $T$ road bearing west, about 20 feet from sign of Warwick Hotel; iron post stamped "Prim. Trav. Sta. No. 15 C, 1918 ".

\begin{tabular}{|rrr|rrr|r}
37 & 12 & 23.47 & 76 & 36 & 19.10 & $\ldots \ldots . .$. \\
& & & & & & \\
37 & 12 & 25.58 & 76 & 36 & 36.16 & 320 \\
37 & 12 & 27.36 & 76 & 35 & 57.30 & 1,537 \\
& 0 & & & & & \\
37 & 12 & 28.72 & 76 & 35 & 51.76 & 470 \\
& & & & & & \\
37 & 12 & 32.07 & 76 & 35 & 17.08 & 2,826 \\
37 & 12 & 47.41 & 76 & 34 & 46.38 & 2,929 \\
37 & 12 & 47.01 & 76 & 34 & 32.50 & 1,124 \\
& & & & & & \\
37 & 12 & 45.78 & 76 & 34 & 19.44 & 1,064
\end{tabular}

South along highways near center of quadrangle. (By J. J. Phelan in 1918.)

Halsteads Point, Prim. Trav. Sta. No. 13 C, 1918 (previously

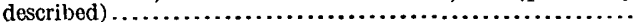

Road fork, 33 feet from telephone poile 2736,6 feet east of road bank, 20 feet southwest from stump on road bank, marked

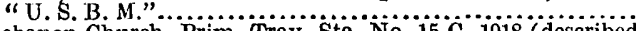

Lebanon Church, Prim. Trav. Sta. No. 15 C, 1918 (described above)...

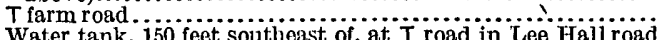
going to depot, in southeast corner of lot; iron post stamped "Prim Trav. Sta. No. 31 P, 1918".

Hub with nail, 44 feet from telephone pole $2804 \ldots \ldots, 21.9$ feet from gatepost east of road, 24.6 feet from cedar trce on west side of road, 30.7 feet from cedar tree on east side of road, 15 feet from barbed-wire fence.

..................................

Fub in center of

near ordnance material storeroom and truck park.............

Crossroads, 50 feet south of 'T. H. Fleming's store door, $25 \mathrm{feet}$ east of sweet-gum tree, 35 feet from telephone pole 2828; hub

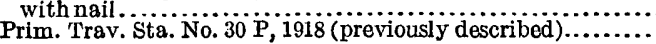

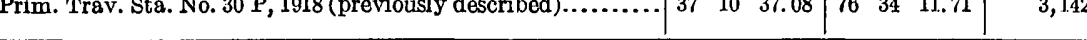

Magnetic declination for center of quadrangle, $5^{\circ} 26^{\prime} \mathrm{W}$.

Along highways. (By J. J. Charters in 1918.)

Prim. Trav. Sta. No. 16 (previously described)...

Station $286,14.22$ feet from 2 -inch iron pipe (landmark), 2 by 4

inch wooden hub

In southeast corner of clearing, about 250 feet east of brown painted house of $F$. C. Lee, about 15 feet west of wooden gate;

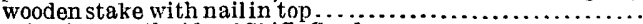

Station 296, north side of Skiffs Creek......................

\begin{tabular}{lll|rrr|r}
37 & 13 & 43.63 & 76 & 34 & 24.13 & $\ldots \ldots \ldots$ \\
37 & 12 & 55.41 & 76 & 34 & 15.57 & 4,926 \\
37 & 12 & 45.78 & 76 & 34 & 19.44 & 1,023 \\
37 & 12 & 01.37 & 76 & 34 & 24.34 & 4,509 \\
& & & & & & \\
37 & 11 & 40.30 & 76 & 34 & 18.86 & 2,177 \\
& & & & & & \\
37 & 11 & 37.37 & 76 & 34 & 18.31 & 300 \\
37 & 11 & 32.46 & 76 & 34 & 18.42 & 498 \\
37 & 11 & 31.45 & 76 & 34 & 18.46 & 102 \\
& &. & & & & \\
37 & 11 & 07.45 & 76 & 34 & 19.83 & 2,429 \\
37 & 10 & 37.08 & 76 & 34 & 11.71 & 3,142 \\
\hline
\end{tabular}

102

429

(1)


YORKTOWN QUADRANGLE-Continued.

Near Skdfis Creek.

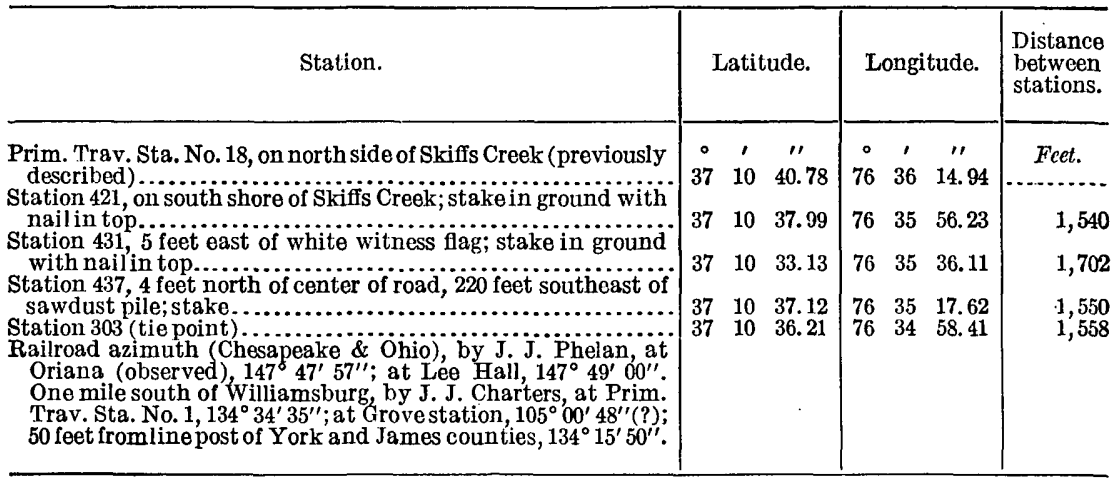

From Lee Hall east and north along highways. (By J. J. Phelan in 1918.)

Hub with nail, 44.1 feet from telephone pole 2804 AA (previously described).

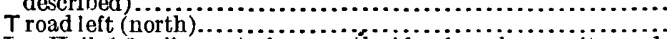

Lee Hall, 1.3 miles east of, on north side of road, opposite road south, 15 feet north of pine tree painted "U. S. 40.6 B. M."; iron post stamped "Prim. Trav. Sta. No. $35 \mathrm{P}, 1918$ " $\mathrm{T}$ road left (north), hub with nail about 4 inches high from
$\mathrm{T}$ ground...

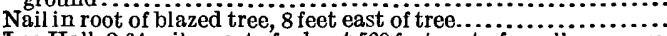

Lee Hall, 2.64 miles east of, a bout 500 feet east of small swampy drain south, in center of fork of woods road, in south root of pine tree 1.6 feet in diameter; iron nail and copper washer,

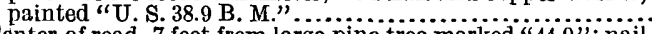

Center of road, 7 feet from large pine tree marked " 44.0 "; nail

in hub........................................

in hub...................................................

road south of upturned pine treo.

Intersection of T roads, hub with nail

Lee Hall, 3.3 miles east of, about 300 feet west of wooden bridge over Great Run, on north side of road forks, blazed pine tree 1.5 feet in diameter, in south root of; iron nail and copper washer, painted "U. S. 41.4 B. M." $\ldots \ldots \ldots \ldots \ldots \ldots \ldots \ldots \ldots$

Intersection of $T$ road, 30 feet from, " 41.8 " painted in white on

pine tree; hub with nail.....................................

Lee Hall, 2.1 miles northeast of, 0.1 mile south of York and Warwick county line, directly opposite $T$ road northeast, on south side of road; iron post stamped "Prim. Trav. Sta. No. 34

Lee Hall, 2.5 miles northeast of, in northeast corner of $\mathrm{T}$ road north, in north root of walnut tree 2.3 feet in diameter; iron nail and copper washer, painted "U. S. 50.8 B. M."...........

Center of $T$ road, 50 feet north of gatepost with heavy chains;

hub with nail.

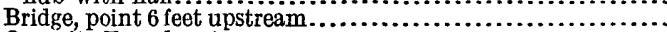

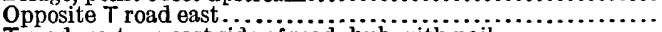

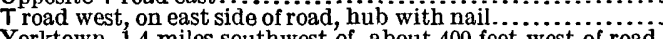

Yorktown, 1.4 miles southwest of, about 400 feet west of road

forks, on west side of road 200 feet west of white frame house

in north root of pine tree 1.8 feet in diameter; iron nail and

copper washer, painted "U. S. 67.2 B. M." $* \ldots ; \% \ldots \ldots \ldots \ldots$

$T$ road west, 10 feet south of pine tree marked " $61 ", \ldots \ldots \ldots \ldots$.

Intersection of $T$ road east, nailin hub................

Yorktown, 1.7 miles southwest of, 0.15 mile east of $\mathrm{N}$. $\mathrm{Dabney}$ 's

farm residence, 50 feet east of road forks, on north side of road, in south root of pine tree 1.4 feet in diameter; iron spike, painted "U. S. 67.4 B. M."

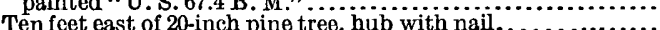

Fifteen feet from pine tree with faded trespass sign, hub with

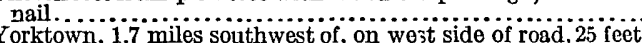

Yorktown, 1.7 miles southwest of on west side of road, 25 reet north of gateway through lane leading to M. Dabney's farm residence; iron post stamped "Prim. Trav. Sta. No. 32 P

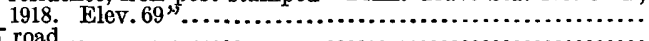

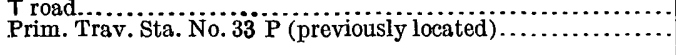

Magnetic declination along north border of quadrangle, $5^{\circ} 28^{\prime} \mathrm{W}$. 
YORKTOWN QUADRATGLE-Continued.

Near Grove triangulation station. (By J. J. Charters in 1918.)

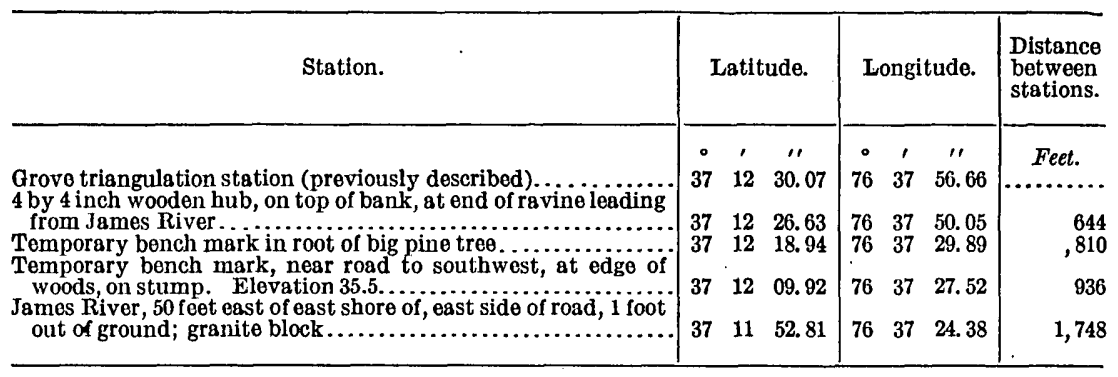

South and west along highways. (By J. J. Phelan In 1918.)

\begin{tabular}{|c|c|c|c|c|c|c|c|}
\hline 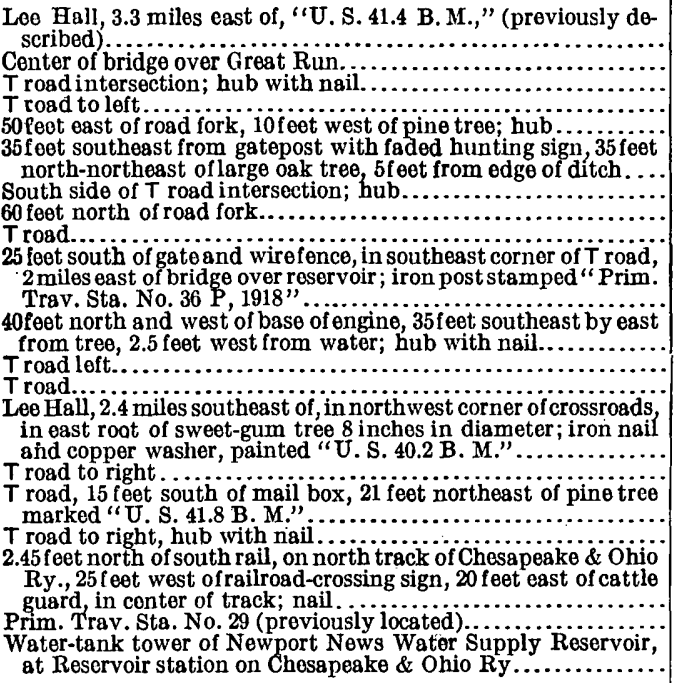 & $\begin{array}{l}37 \\
37 \\
37 \\
37 \\
37 \\
\\
37 \\
37 \\
37 \\
37\end{array}$ & $\begin{array}{l}11 \\
11 \\
11 \\
11 \\
11\end{array}$ & $\begin{array}{l}43.74 \\
43.86 \\
48.36 \\
50.22 \\
08.37 \\
\\
03.37 \\
00.01 \\
49.93 \\
42.58\end{array}$ & $\begin{array}{l}76 \\
76 \\
76 \\
76 \\
76 \\
\\
76 \\
76 \\
76 \\
76\end{array}$ & $\begin{array}{l}31 \\
31 \\
\mathbf{3 1} \\
\mathbf{3 1} \\
\mathbf{3 1}\end{array}$ & $\begin{array}{l}57.00 \\
06.96 \\
08.59 \\
12.50 \\
\\
51.17 \\
57.38 \\
52.86 \\
20.25 \\
\\
22.57 \\
25.83\end{array}$ & $\begin{array}{r}352 \\
942 \\
719 \\
4,255 \\
792 \\
784 \\
874 \\
1,027 \\
808 \\
\\
3,165 \\
\\
430 \\
793 \\
2,370 \\
\\
\\
244 \\
328\end{array}$ \\
\hline
\end{tabular}

East along htghways to Orlana. (Unchecked spur line.)

Prim. Trav. Sta. No. 27 P, 1918 (previously located).

Oriana station, 500 foet north of, in center of railroad track, in westbound track, 25 feet southeast of railroad-crossing sign-

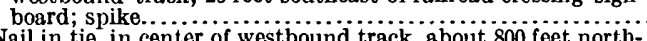

Nail in tie, in center of westbound track, about 800 feet northwesterly from previous position.........................

Magnetic declination in northeast corner of quadrangle, $5^{\circ} 33^{\prime} \mathrm{W}$.

\begin{tabular}{rrr|rrr|r}
37 & 07 & 38.54 & 76 & 32 & 22.00 & $\ldots \ldots \ldots$ \\
37 & 08 & 05.72 & 76 & 31 & 35.95 & 4,633 \\
37 & 08 & 12.41 & 76 & 31 & 41.21 & 800 \\
\hline
\end{tabular}

$$
\text { 121166 }{ }^{\circ}-23-\text { Bull. } 709 \longrightarrow 33
$$




\title{
TRIANGULATION STATIONS.
}

\section{Yorktown quadrangle.}

\author{
WARWICK COUNTY.
}

CAMP ABRAHAM EUSTIS.

The following geographic positions on North American datum were determined by primary traverse and are based upon Coast and Geodetic Survey triangulation station Crawford and U. S. Geological Survey primary traverse stations determined in 1918.

Azimuths and distances were obtained from inverse computations from previously determined latitudes and longitudes.

The work was done by F. J. McMaugh in 1919:

TOWER NO. 1, WARWICK COUNTY, VA.

Motor Transport School, 900 feet south of curve in road at; stake at center of site of steel observation tower 105 feet high.

[ Latitude $37^{\circ} 09^{\prime} 00.14^{\prime \prime}$. Longitude $76^{\circ} 35^{\prime} 20.94^{\prime \prime}$.]

\begin{tabular}{|c|c|c|c|}
\hline To station- & Azimuth. & Back azimuth. & Distance. \\
\hline 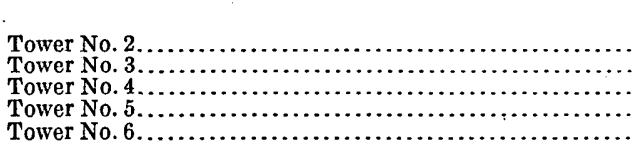 & $\begin{array}{ccc}\circ & \prime & \prime \prime \\
49 & 20 & 24 \\
336 & 58 & 48 \\
114 & 56 & 03 \\
336 & 37 & 44 \\
145 & 53 & 56\end{array}$ & $\begin{array}{ccc}\circ & \prime & \prime \prime \\
229 & 18 & 18 \\
156 & 59 & 32 \\
194 & 55 & 36 \\
156 & 39 & 05 \\
325 & 51 & 43\end{array}$ & $\begin{array}{r}\text { Log. meters. } \\
\text { 3. 5549473 } \\
3.6640887 \\
3.6305239 \\
3.9226465 \\
3.9854945\end{array}$ \\
\hline
\end{tabular}

TOWER NO. 2, WARWICK COUNTY, VA.

Mulberry Point, 0.50 mile northeast of, 120 feet northeast of Coast and Geodetic Survey triangulation tablet in concrete post on shore rampart of old Fort Crawford; stake at center of site for steel observation tower.

[Latitude $37^{\circ} 07^{\prime} 44.59^{\prime \prime}$. Longitude $76^{\circ} 37^{\prime} 11.23^{\prime \prime}$.]

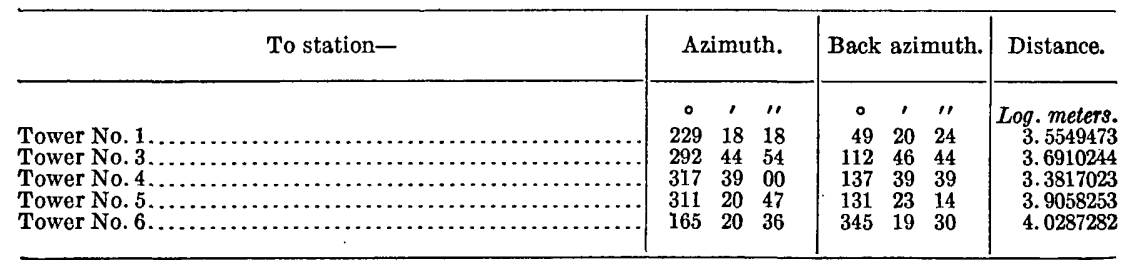


TOWER NO. 3, WARWICK COUNTY, VA.

Curtis Point, 1.90 miles northwest of, 0.60 mile east of main road on Mulberry Island, 200 feet east of site of farmhouse, on west bank of Warwick River; stake at center of site for steel observation tower.

[Latitude $37^{\circ} 06^{\prime} 42.97^{\prime \prime}$. Longitude $76^{\circ} 34^{\prime} 07.86^{\prime \prime}$.]

\begin{tabular}{|c|c|c|c|c|c|c|}
\hline To station- & \multicolumn{2}{|c|}{ Azimuth. } & \multicolumn{3}{|c|}{ Back azimuth. } & Distance. \\
\hline 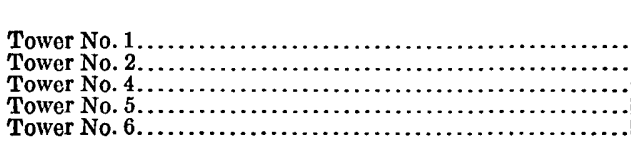 & $\begin{array}{r}\circ \\
156 \\
112 \\
92 \\
336 \\
149\end{array}$ & $\begin{array}{ll}5 & 1 \prime \\
59 & 32 \\
46 & 45 \\
22 & 04 \\
07 & 04 \\
26 & 49\end{array}$ & $\begin{array}{l}0 \\
336 \\
292 \\
272 \\
156 \\
329\end{array}$ & $\begin{array}{l}1 \\
58 \\
44 \\
20 \\
07 \\
23\end{array}$ & $\begin{array}{l}48 \\
54 \\
53 \\
42 \\
52\end{array}$ & $\begin{array}{r}\text { Log. meters. } \\
\text { 3. } 6640887 \\
\text { 3. } 6910244 \\
\text { 3. } 4635208 \\
\text { 3. } 5729940 \\
\text { 4. } 1526472\end{array}$ \\
\hline
\end{tabular}

TOWER NO. 4, WARWICK COUNTY, VA.

Morrison Creek, 400 feet east of point at mouth of, 800 feet southwest of site of old farm buildings; stake at center of site for steel observation tower.

[Latitude $37^{\circ} 06^{\prime} 46.85^{\prime \prime}$. Longitude $76^{\circ} 36^{\prime} 05.52^{\prime \prime}$.]

\begin{tabular}{|c|c|c|c|c|}
\hline To station- & Azimuth. & Back & azimuth. & Distance. \\
\hline 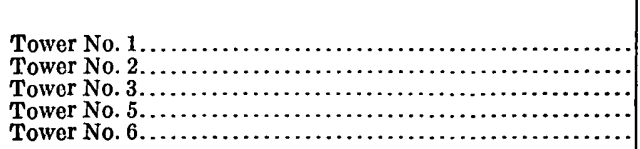 & $\begin{array}{rcc}\circ & \prime & \prime \prime \\
194 & 55 & 36 \\
137 & 39 & 39 \\
92 & 22 & 04 \\
308 & 40 & 53 \\
160 & 21 & 48\end{array}$ & $\begin{array}{c}\circ \\
14 \\
317 \\
272 \\
128 \\
340\end{array}$ & $\begin{array}{cc}\prime & \prime \prime \\
56 & 03 \\
39 & 00 \\
20 & 53 \\
42 & 41 \\
20 & 03\end{array}$ & $\begin{array}{r}\text { Log. meters. } \\
3.6305239 \\
3.3817023 \\
3.4635208 \\
3.7530488 \\
4.1094106\end{array}$ \\
\hline
\end{tabular}

TOWER NO. 5, WARWICK COUNTY, VA.

Curtis Point, near end of, 130 feet southeast of old gateway; stake at center of site for steel tower.

[Latitude $37^{\circ} 04^{\prime} 52.00^{\prime \prime}$. Longitude $76^{\circ} 33^{\prime} 06.54^{\prime \prime}$.]

\begin{tabular}{|c|c|c|c|c|}
\hline To station- & Azimuth. & Back & azimuth. & Distance. \\
\hline 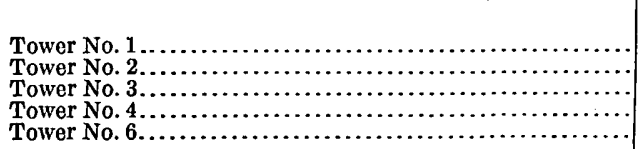 & $\begin{array}{ccc}\circ & , & \prime \prime \\
156 & 39 & 05 \\
131 & 23 & 14 \\
156 & 07 & 42 \\
128 & 42 & 41 \\
150 & 50 & 47\end{array}$ & $\begin{array}{l}\circ \\
336 \\
311 \\
336 \\
308 \\
330\end{array}$ & $\begin{array}{cc}\prime & \prime \prime \\
37 & 44 \\
20 & 47 \\
07 & 04 \\
40 & 53 \\
47 & 13\end{array}$ & $\begin{array}{r}\text { Log. mcters. } \\
\text { 3. } 9226465 \\
\text { 3. } 9058253 \\
3.5729940 \\
3.7530488 \\
\text { 4. } 2536414\end{array}$ \\
\hline
\end{tabular}

TOWER NO. 6, WARWICK COUNTY, VA.

J. D. Dunn's house, about 500 feet southeast of, 0.25 mile south of main road, near ravine, 300 feet southeast of iron post stamped "Prim. Trav. Sta. No. 4"; stake at center of site for steel observation tower.

[Latitude $37^{\circ} 13^{\prime} 19.87^{\prime \prime}$. Longitude $76^{\circ} 39^{\prime} 00.88^{\prime \prime}$.]

\begin{tabular}{|c|c|c|c|c|c|c|c|}
\hline To station- & \multicolumn{3}{|c|}{ Azimuth. } & \multicolumn{3}{|c|}{ Back azimuth. } & \multirow{2}{*}{\begin{tabular}{|} 
Distance. \\
Log. meters. \\
3.9854945 \\
4.0287282 \\
4.1526472 \\
4.1094106 \\
4.2536414
\end{tabular}} \\
\hline 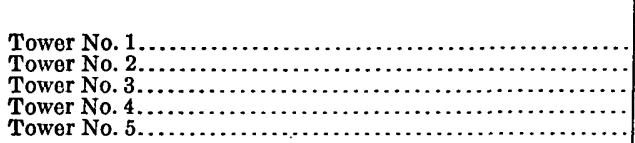 & $\begin{array}{l}3 \\
325 \\
345 \\
329 \\
340 \\
330\end{array}$ & $\begin{array}{l}1 \\
51 \\
19 \\
23 \\
20 \\
47\end{array}$ & $\begin{array}{l}\prime \prime \\
43 \\
30 \\
52 \\
03 \\
13\end{array}$ & $\begin{array}{l}\circ \\
145 \\
165 \\
149 \\
160 \\
150\end{array}$ & $\begin{array}{l}, \\
53 \\
20 \\
26 \\
21 \\
50\end{array}$ & $\begin{array}{l}1 \prime \\
56 \\
36 \\
49 \\
48 \\
47\end{array}$ & \\
\hline
\end{tabular}


